\title{
Pattern Formation in Spatially Forced Thermal Convection
}

\author{
Dissertation \\ zur Erlangung des mathematisch-naturwissenschaftlichen Doktorgrades \\ ,Doctor rerum naturalium “ \\ an der Georg-August-Universität Göttingen \\ vorgelegt von
}

Stephan Weiß

aus Suhl

Göttingen 2009 
Referent: Prof. Dr. E. Bodenschatz

Koreferent: Prof. Dr. A. Tilgner

Tag der mündlichen Prüfung: 14. Oktober 2009 


\section{Contents}

1 Introduction $\quad 15$

1.1 Turing's Legacy . . . . . . . . . . . . . . . . . . . 15

1.2 Basic Pattern Formation . . . . . . . . . . . . . . . . . . 17

1.3 Convection Driven by a Thermal Gradient . . . . . . . . . . . . . 21

1.4 Pattern Formation in Anisotropic Systems . . . . . . . . . . . . . 25

1.5 Temporal and Spatial Periodic Forcing of Pattern Forming Systems 27

2 Theoretical Overview 31

2.1 Boussinesq Equations . . . . . . . . . . . . . . . . . . . 31

2.2 Surface Corrugation . . . . . . . . . . . . . . . . . . . . 33

2.3 Inclined Layer Convection (ILC) . . . . . . . . . . . . . 36

2.4 Numerical Methods . . . . . . . . . . . . . . . . . . . . . . . . . 42

3 The Experiment $\quad 45$

3.1 Overview of the Gas Convection Apparatus . . . . . . . . . . . 45

3.2 Surface Corrugation . . . . . . . . . . . . . . . . . . . . . . . 49

3.3 Visualization . . . . . . . . . . . . . . . . . 55

3.3.1 Shadowgraphy . . . . . . . . . . . . . 55

3.3.2 Image Processing . . . . . . . . . . . . . . . . . 61

3.3.3 Some Remarks about Fourier Analysis . . . . . . . . . . 65

3.3.4 Phase Demodulation . . . . . . . . . . . . . . . 69

4 Inclined and Noninclined Resonant Forcing $\quad 73$

4.1 Forced Rolls and Amplitude Equation . . . . . . . . . . . . . . . 73

4.2 Longitudinal Forcing . . . . . . . . . . . . . . . . . . . 77

4.2.1 Varicose Pattern . . . . . . . . . . . . . . . 80 
4.2.2 Harmonic Undulation and Crawling Rolls . . . . . . . . . . 84

4.2.3 Patterns Driven by Shear Instability . . . . . . . . . . 88

4.2.4 Patterns at Low Inclination Angles . . . . . . . . . . . . 95

4.3 Transverse Forcing . . . . . . . . . . . . . . . . . . . . . . 99

4.3.1 Rhombic Pattern . . . . . . . . . . . . . . . 100

4.3.2 Hexarolls and Crawling Rolls . . . . . . . . . . . . 106

4.3.3 Bimodal Pattern . . . . . . . . . . . . . . . . 110

4.3.4 Scepter Pattern . . . . . . . . . . . . . . . . . 113

4.3 .5 Heart Pattern . . . . . . . . . . . . . . . . . . 119

4.4 Forcing Oblique Rolls: $\varphi=60^{\circ} \ldots$. . . . . . . . . . . 120

5 Forcing with Various Wave Numbers $\quad 129$

5.1 Varicose Pattern . . . . . . . . . . . . . . . . . . . 131

5.2 Coherent Kinks . . . . . . . . . . . . . . . . . . . . . . . . . . . . 134

5.3 Cross Rolls . . . . . . . . . . . . . . . . . . . . . 138

5.4 Brickwall Pattern . . . . . . . . . . . . . . . . . . . 142

5.5 The Phase Diagram . . . . . . . . . . . . . . . . . . 144

6 Optical Forcing - An Outlook 149

6.1 Theory . . . . . . . . . . . . . . . . . . 150

6.2 The Experiment . . . . . . . . . . . . . . . . . . . . . 152

6.3 Results and Discussion . . . . . . . . . . . . . . . . . 154

6.3.1 Spatial Periodic Forcing and the Amplitude Equation . . . 154

6.3 .2 Forcing Close to $R a_{c} \ldots \ldots \ldots$. . . . . . . 156

6.4 More Power - The Use of an IR Laser. . . . . . . . . . . . . . . . 159

$\begin{array}{lll}7 & \text { Summary } & 163\end{array}$

$\begin{array}{ll}\text { A Boussinesq Equations } & 167\end{array}$

A.1 Unforced ILC . . . . . . . . . . . . . . . . . . . . . . . 167

A.2 Forced ILC: . . . . . . . . . . . . . . . . . . . . . . . . 168

$\begin{array}{lll}\text { B Filling and emptying procedure for } \mathrm{CS}_{2} & 171\end{array}$

$\begin{array}{ll}\text { C Suplementary Material on CD-ROM } & 173\end{array}$ 
C.1 Movies . . . . . . . . . . . . . . . . . . . . 173

C.2 RBC - Program to calculate fluid properties . . . . . . . . 174 


\section{List of Figures}

1.1 Turing pattern on the pelt of a jaguar . . . . . . . . . . . 16

1.2 Neutral curve and neutral surface for the Swift-Hohenberg equation 20

1.3 Pitchfork bifurcation . . . . . . . . . . . . . . . . . . 21

1.4 Schematic of a convection cell with periodic fluid motion . . . . . 22

1.5 Neutral curve for Rayleigh Bénard convection . . . . . . . . . . . 24

1.6 Stability regions for ISR at different Prandtl numbers . . . . . . . 24

1.7 Inclined layer convection (ILC) $\ldots \ldots$. . . . . . . . . 26

1.8 Pitchfork bifurcation and imperfect bifurcation . . . . . . . . . . 29

2.1 Side and top view of an inclined convection cell (schematic). . . . 37

2.2 Onset of straight rolls as a function of $\gamma$ and $\varphi$. . . . . . . . . 40

2.3 Critical wave number as a function of $\gamma$ and $\varphi \ldots . . . . . .41$

3.1 Sketch of the convection apparatus . . . . . . . . . . 46

3.2 Shadowgraph image of the forced and the unforced cell . . . . . . 47

3.3 Overview of the experimental apparatus . . . . . . . . . . 50

3.4 Schematic of the bottom plate surface . . . . . . . . . . . . 51

3.5 Schematic drawing of the shadowgraph arrangement . . . . . . . 57

3.6 Light rays and light intensity due to a sinusoidal refraction grating 59

3.7 Shadowgraph intensity in dependence on the axial distance . . . . 60

3.8 Shadowgraph intensity due to bottom plate texture . . . . . . . . 62

3.9 Standard deviation of the intensity in dependence on the shadowgraph setting . . . . . . . . . . . . . . . 64

3.10 Standard deviation of the shadowgraph signal for forced and unforced convection in dependence of the control parameter $\varepsilon$. . . 65

3.11 Shadowgraph images of the forced and the reference cell at different $\varepsilon 66$ 
3.12 Consideration of the amplitude of specific modes . . . . . . . . . . 68

3.13 Demodulation of stripe pattern . . . . . . . . . . . 70

3.14 Demodulation of Varicose Pattern . . . . . . . . . . . . . . . 71

4.1 Schematic of the convection cell for forced ILC . . . . . . . . . . . 74

4.2 Convection amplitudes for forced and unforced convection as a

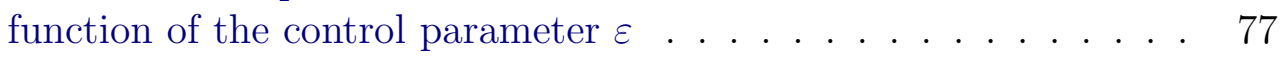

4.3 Phase diagram for longitudinal forcing . . . . . . . . . . . . . 79

4.4 Straight rolls in unforced and forced convection experiments . . . 80

4.5 Varicose pattern . . . . . . . . . . . . . . . 81

4.6 Calculated varicose pattern . . . . . . . . . . . . . 82

4.7 Modulation of the phase field of varicose pattern . . . . . . . . . . 82

4.8 Spatio-temporal chaotic state for horizontal convection and its corresponding Fourier transform . . . . . . . . . . . 83

4.9 Harmonic undulations . . . . . . . . . . . . . . . . . . . . 84

4.10 Undulation pattern and its phase field . . . . . . . . . . . 86

4.11 Undulation chaos and crawling rolls . . . . . . . . . . . . . 89

4.12 Time evolution of transverse bursts (TB) . . . . . . . . . . . 90

4.13 Fourier transform transverse bursts . . . . . . . . . . . . . . . 91

4.14 Amplitude of transverse bursts as a function of time . . . . . . . 92

4.15 Transverse rolls and their Fourier transform . . . . . . . . . . 93

4.16 Fourier-filtered transverse rolls after removing the forced mode $\vec{q}_{f} \quad 94$

4.17 Time evolution of a longitudinal burst . . . . . . . . . . . . 94

4.18 Single kink line, subharmonic resonances and kink cluster. . . . . 96

4.19 Varicose pattern at higher inclination angles . . . . . . . . . . 97

4.20 Crawling kinks . . . . . . . . . . . . . . . . . . . . . . . 98

4.21 Localized crawling kinks . . . . . . . . . . . . . . . . . . . 99

4.22 Phase diagram (transverse forcing) . . . . . . . . . . . . . . 101

4.23 Longitudinal rolls at high $\varepsilon \ldots \ldots$. . . . . . . . . . . . . . . . 102

4.24 Evolution of rhombic pattern with time at very small inclination angles . . . . . . . . . . . . . . . . 103

4.25 Fourier transform of varicose instability during evolution of rhombic pattern . . . . . . . . . . . . . . 103

4.26 Rhombic pattern and its Fourier transform . . . . . . . . . 104 


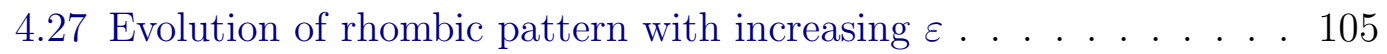

4.28 Rhombic pattern at $\gamma=5^{\circ}$ and $\varepsilon=3.7 \ldots$. . . . . . . 106

4.29 Hexarolls and its corresponding Fourier transform . . . . . . . . . 107

4.30 Calculated hexaroll pattern . . . . . . . . . . . . 108

4.31 Hexarolls and the transition to crawling rolls . . . . . . . . . . . . 109

4.32 Bimodal pattern and its corresponding Fourier transform . . . . . 110

4.33 Mode evolution of bimodal pattern . . . . . . . . . . . . . . . 112

4.34 Bimodal pattern, calculated with Eq. 4.10 . . . . . . . . . 112

4.35 Scepter pattern . . . . . . . . . . . . . . . . . . 113

4.36 Scepter pattern as a convolution . . . . . . . . . . . . 114

4.37 Amplitude development of scepter pattern for several modes . . . 116

4.38 Fourier decomposition of scepter pattern . . . . . . . . . . . . 118

4.39 Calculated scepter pattern . . . . . . . . . . . . . . . 120

4.40 Heart pattern . . . . . . . . . . . . . . . . . . . 121

4.41 Decomposition of Heart pattern . . . . . . . . . . . . . . . 121

4.42 Comparison between switching diamond panes and heart pattern . 122

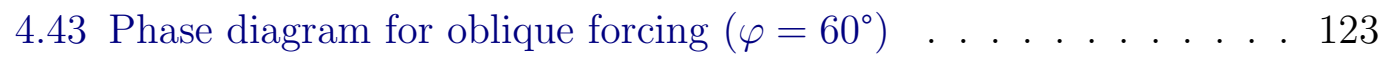

4.44 Kinks and oblique longitudinal rolls . . . . . . . . . . . . . 124

4.45 Rhombic and oblique hexarolls . . . . . . . . . . . . . . . 125

4.46 Transition from oblique bimodal to oblique hexarolls . . . . . . . 126

4.47 Development of crawling rolls . . . . . . . . . . . . . . . . 127

4.48 Pretzel pattern . . . . . . . . . . . . . . . . . 127

5.1 Busse balloon for $\operatorname{Pr}=1.27$. . . . . . . . . . . . . . . 130

5.2 Straight rolls, Varicose Pattern and spatio-temporal chaos at $q_{f}=$ $1.08 q_{c} \ldots \ldots \ldots \ldots \ldots 131$

5.3 Development of the varicose mode $\vec{q}_{1}$ as a function of $q_{f} \ldots \ldots 132$

5.4 Amplitude evolution of the varicose modes for $q_{f}=1.08 q_{c}$. . . . 133

5.5 Kinks and their phases . . . . . . . . . . . . . . 135

5.6 Comparison between kinks and skewed varicose . . . . . . . . . 136

5.7 Kink lines for different forcing wave numbers . . . . . . . . . . . 136

5.8 Development of kink lines as a function of $q_{f} / q_{c} \ldots \ldots$. . . . . 138

5.9 Length scales in x-direction and typical angle of VP and kinks . . 139

5.10 Cross roll pattern . . . . . . . . . . . . . . . . 140 
5.11 Transition from cross rolls to spatio-temporal chaos . . . . . . . . 141

5.12 Brickwall pattern and its corresponding Fourier transform . . . . 142

5.13 Small areas of hexagons in the unforced reference cell due to nonBoussinesq effects . . . . . . . . . . . . . . . . 143

5.14 Development of brickwall pattern . . . . . . . . . . . . . . 144

5.15 Instability of forced rolls with various wave numbers . . . . . . . . 146

6.1 Temperature profiles due to internal heating . . . . . . . . . . . . 151

6.2 Experimental setup of an optical forcing experiment . . . . . . . . 153

6.3 Imperfect bifurcation due to spatial periodic internal heating . . . 155

6.4 Sequence of shadowgraph images during a fast increase of the temperature difference . . . . . . . . . . . . . . . . 157

6.5 Convection pattern due to optical forcing . . . . . . . . . . . 160

6.6 Schematic drawing of the $\mathrm{CS}_{2}$ filling system . . . . . . . . . . 162

7.1 Banded vegetation in arid regions . . . . . . . . . . 165 


\section{Symbols and Abbreviations}

$\Delta T$ temperature difference between top and bottom plate

$\Delta T_{c}$ critical temperature difference for the onset of convection rolls in an unforced system

$\gamma \quad$ inclination angle

$\kappa \quad$ thermal diffusivity

$\nu \quad$ kinematic viscosity

$\operatorname{Pr} \quad$ Prandtl number

$R a \quad$ Rayleigh number

$R a^{I}=\cos (\gamma) R a \ldots$ Rayleigh number in inclined layer convection

$\rho \quad$ dimensionless grating strength parameter as defined in Sec. 3.3.1

$\theta \quad$ deviation of the temperature field from $T_{\text {cond }}$

$\varrho \quad$ mass density

d cell height

$q_{c}, \lambda_{c}$ critical wave number and critical wavelength, at which the neutral curve has its minimum

$q_{f}, \lambda_{f}$ forcing wave number and forcing wavelength

$T_{0} \quad=\frac{T_{b}+T_{t}}{2} \ldots$ averaged temperature

$t_{\kappa} \quad=d^{2} / \kappa \ldots$ vertical thermal diffusion time

$T_{b} \quad$ temperature at the bottom plate

$T_{t} \quad$ temperature at the top plate

$T_{\text {cond }}$ temperature distribution in the purely conductive regime 
$T_{\text {mod }}$ term of the temperature field which contains the temperature modulation

$z_{\max }^{g}$ distance between the convection cell and the imaging plane at which a caustic would occur regarding geometrical optic

$z_{\max }^{p}$ distance between the convection cell and the imaging plane where regarding physical optics a first maxima exists

BM Bimodal pattern

CR Crawling rolls

HP Heart pattern

HR Hexarolls

ILC Inclined Layer Convection

KL Kink lines

LB Longitudinal bursts

LR Longitudinal rolls

OB Oblique bimodals

OR Oblique rolls

OX Oblique hexarolls

PZ Pretzel pattern

RBC Rayleigh-Bénard Convection

RO Rhombic pattern

SP Scepter pattern

TB Transverse bursts

TR Transverse rolls

VP Varicose pattern 
Les formes de courants que j'ai ainsi pu observer dans des liquides n'offrant d'autre hétérogénéité que des différences de températures, présentent, si je ne me trompe, l'intérêt tout spécial et nouveau d'un phénomène physique, remarquablement simple, créant de toutes pièces cette structure cellulaire qui, jusqu'à présent, semblait particulière aux êtres vivants et caractéristique du monde organique.

The shape of the currents I was able to observe in liquids offering no other heterogeneity than temperature differences are, if I am not mistaken, especially interesting and novel in that they are examples of remarkably simple physical phenomena able to create from the scratch a cellular structure that seemed, up until now, to be particular to living beings and characteristic of the organic world.

Henri Bénard, 1900 



\section{Chapter 1}

\section{Introduction}

\section{$1.1 \quad$ Turing's Legacy}

In 1952, Alan Turing, the brilliant British mathematician, suggested a model to describe morphogenesis, the differentiation process which forms an embryo out of a cell cluster [2]. This was especially remarkable since during that period modern molecular biology had just begun. Not until a year later did Francis Crick and James Watson reveal the structure of the DNA-molecule [3]. Turing's model was astonishingly simple and only a theoretical construct, but nevertheless it turned out over the following years to be very successful, not only in describing simple patterns on the skin and pelts of animals, but also in understanding pattern forming processes in general. It is remarkable that out of a very simple model, a large variety of different static and dynamical patterns can occur. Although almost forty years had to pass until the first Turing pattern was observed experimentally [4], his influence to the scientific community was enormous [5].

Turing was trying to answer the question of how a cluster of identical cells with identical genetic information differentiate and form specialized cells, tissues and organs. And furthermore, at which point cells "know" whether they should become a skin cell or part of the heart tissue, for example. The genetic code itself does not contain any information about it. Turing assumed that certain molecules, so called morphogenes, influence the expression of genetic information and their realization in a cell. Genes which control the further development of the cell are either activated or deactivated, depending on the concentration of a specific morphogen.

In Turing's model, only two kinds of molecules are involved. The first one, called the activator, is autocatalytic, meaning it stimulates its own production. On the other hand it also stimulates a second reaction, in which a second molecule is produced - the inhibitor. The inhibitor reduces the reaction rate of both reactions. Therefore, a high concentration of activator leads to a high reaction 


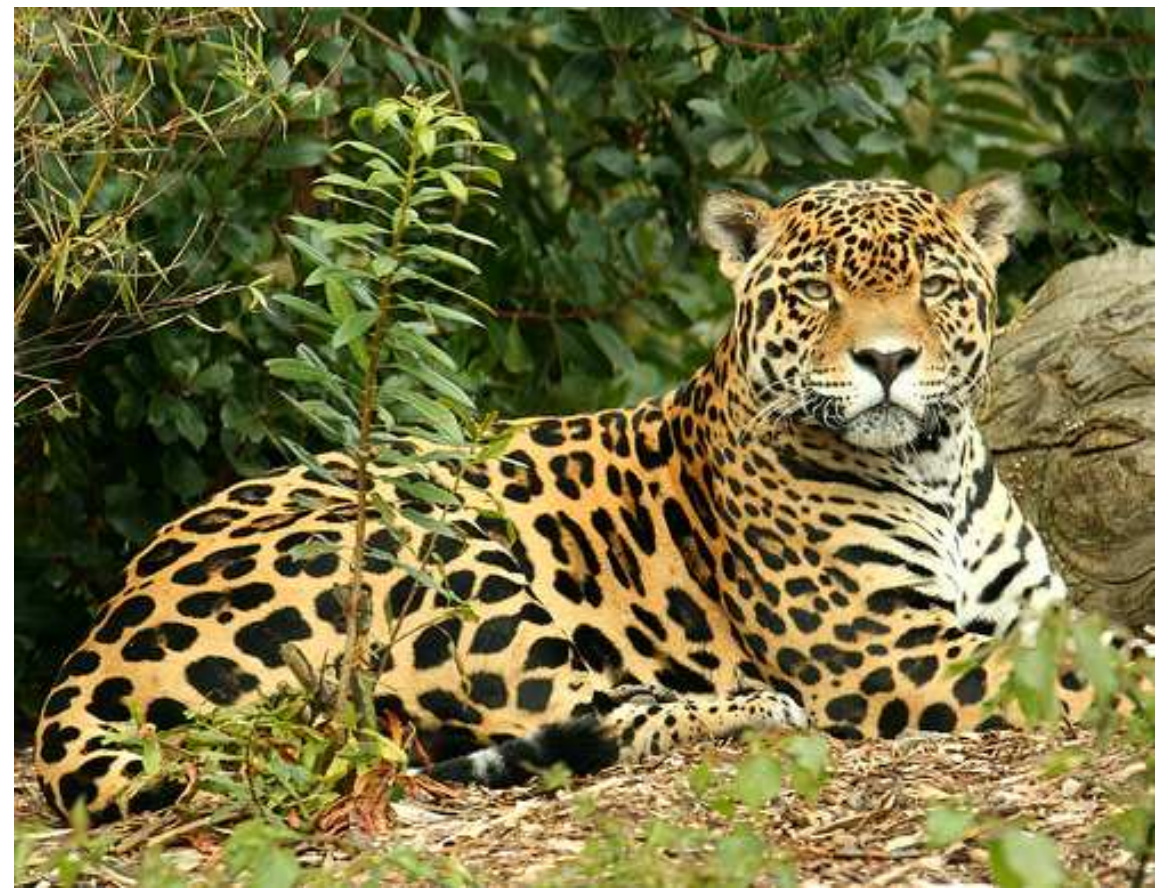

Figure 1.1: It is suggested, that a Turing-mechanism is responsible for the pattern on pelts and skins of the jaguar (Panthera onca) and other animals (copyright: public domain).

rate, while a high concentration of the inhibitor leads to a lower reaction rate. In this way the production rates of the two molecules depend strongly on their relative concentrations.

Both chemicals diffuse from cell to cell with different diffusion rates ${ }^{1}$. If the inhibitor diffuses faster than the activator, the inhibitor concentration becomes overwhelming and stops both reactions, at areas further away from regions of high activator concentration. A spatial pattern might be created with alternating areas of high and low activator concentrations. In dependence on the diffusion constants, the exact reaction kinetics, and the boundaries a variety of steady and dynamical pattern can be created by this mechanism.

In recent years, several different kinds of pattern, created by a Turing-like mechanism, were found in chemistry $[4,6,7,8]$ and biology [9, 10]. Nowadays, in the time of modern molecular biology, members of the protein families such as WNT ("wingless int") and DKK ("Dickkopf") are known to function as signal molecules, which influence genetic expressions in cells $[11,12]$. Very recent experiments [13] on mice show that these proteins even act as activator and inhibitor

\footnotetext{
${ }^{1}$ As Turing showed qualitatively it does not matter, whether one consider discrete cells or a continuous medium in which diffusion takes place.
} 
molecules, and thus are an example of morphogenes exactly as Turing described more than 50 years ago. In fact, it is expected that the homogeneous distribution of hair follicle on the skin of animals is driven by a Turing mechanism. By increasing the concentration of the inhibitor molecule in the experiment the averaged area per follicle increased in the same way as predicted by numerical simulations. The distribution of hair follicle and the well known pattern on zebras, shells and leopards, are just very simple realizations of possible patterns. It is assumed that the whole complexity of organisms is caused by only a handful of signal molecules [14]. Without these mechanisms every cell in an organism would be the same.

\subsection{Basic Pattern Formation}

Turing patterns are just one fascinating example, for the formation of complex patterns, as a result of very simple underlying mechanisms. Nowadays a huge variety of different pattern forming systems (PFS) is known. From simple fluid dynamics [15, 16], over neuroscience [17] to economy [18], periodic structures in space and time occur spontaneously out of homogeneous states, without any external ordering force $[15,19]$. In order to understand the complexity in our world it is important to study the basic mechanisms of pattern forming processes.

Surprisingly, nature shows a set of "standard" patterns, like stripes, hexagons or spirals in complete different systems. Sand ripples at the beach, stripes of a zebra or the flow in electroconvection have totally different underlying mechanisms but very similar phenomenologies. Thus, quite often it is possible to describe similar patterns in different systems by similar equations. Even if the underlying mechanisms are not understood in detail, in many cases one can say something about the evolution of the system, or its stability.

Many PFS are far from thermal equilibrium and are driven by a constant energy flux, which obtains its order. Without this flux, regarding the second law of thermodynamics, the system would turn to an equilibrium, whereas any periodicity would disappear resulting in a complete homogeneous state. By controlling the energy flux one can control the state of the system externally and turn it from an isotropic homogeneous state to an inhomogeneous anisotropic one.

Such transitions are usually described by means of control and order parameters. While the control parameter is usually an easy to change parameter in the system, the order parameter describes the state of the system. The order parameter is zero in the homogeneous state and becomes finite when the system turns to the inhomogeneous anisotropic state. Simple equations, that describe the relation between the order parameter and the control parameter can often be derived from the complex governing equations of the system. Control and order parameter are not a special tool to describe pattern forming systems, but are rather well established in describing phase transitions in general [20]. A well known 
example therefor are ferromagnets under the absence of a magnetic field. If the temperature is decreased below a critical point, a transition takes place from the paramagnetic phase, where the spins are randomly oriented, to a ferromagnetic phase with parallel aligned spins. Here the term "order parameter" really characterizes the mean degree of spin order. It is zero, if the spins are random and increases if the spins start to align parallel.

But there are also non-dissipative pattern forming systems that are in thermal equilibrium such as in example the Rosensweig instability [21]. ${ }^{2}$ This instability occurs in ferrofluids - suspensions of 5-10 nm small ferromagnetic particles in a carrier liquid. If one applies a magnetic field on a ferrofluid one observes a hysteretic transition of the free liquid surface, when the magnetic field exceeds a certain critical value $H_{c}$. The formerly flat surface changes spontaneously its shape and spikes occur, sitting on a hexagonal lattice with a well defined wavelength [21]. The control parameter here is the strength of the magnetic field and the height of the spikes can be seen as the order parameter. The transition from an isotropic homogeneous state to a periodic state with a well defined wavelength by changing a control parameter (here the magnetic field) is a very typical characteristic of a pattern forming system.

Although simple processes can create complex patterns, the detailed mechanisms of a PFS are often not known, or the governing differential equations are highly nonlinear and thus not easily solved. However, full solutions of realistic equations are mostly not needed. Thus, one often extracts from each system only these features that are relevant for the pattern forming process and incorporates them in new simpler model equations. One such simple model equation that reproduces several universal features of a PFS is the Swift-Hohenberg equation [23]. Originally suggested to describe thermal convection close to its onset, the Swift-Hohenberg equation (SHE) became very successful in describing pattern forming processes [15] in general. In its simplest form it describes the evolution of a concentration or temperature field $\theta(\vec{r}, t):^{3}$

$$
\frac{\partial \theta}{\partial t}=\left[\varepsilon-\left(\nabla^{2}+q_{c}^{2}\right)^{2}\right] \theta-\theta^{3} .
$$

In this equation, $\varepsilon$ is a control parameter and $q_{c}$ a constant. A solution $\theta=0$ becomes unstable as $\varepsilon$ increases from negative to positive values. We assume a small perturbation of the form

$$
\theta=\theta_{0} e^{\sigma t+\mathrm{i} \vec{q} \cdot \vec{x}}
$$

\footnotetext{
${ }^{2}$ Another example would be a block copolymer, where at sufficiently small temperature the two component segregate and form microscopic domains [22].

${ }^{3}$ Note that this form cannot evolve a hexagonal solution, since it is invariant under a change of the sign of the field. In order to get an equation, which can also describe hexagons, a quadratic term has to be added.
} 
After neglecting non-linear terms, one sees immediately the dependency of the growth rate $\sigma$ on $\varepsilon$ and on the wavelength $q$ :

$$
\sigma=\varepsilon-\left(q^{2}-q_{c}^{2}\right)^{2}
$$

Small perturbations are damped for $\sigma<0$ whereas perturbations grow if $\sigma>0$. For a given wave number $q$, an $\varepsilon$ exists, at which the system is marginal or neutral stable $(\sigma=0)$. The curve with all points $(q, \varepsilon)$ for which the system is marginally stable is termed neutral curve (Fig. 1.2). In isotropic systems, such as approximated by the isotropic Swift-Hohenberg equation (Eq. 1.1) the neutral curve only depends on the modulus of the wave vector $(q)$ and is therefore really a curve. In general, $\varepsilon$ for which the system is marginally stable is a function of the three components of the wave vector $\left(q_{x}, q_{y}, q_{z}\right)$ and therefore the neutral curve is a volume in the otherwise four-dimensional parameter space $\left(\varepsilon, q_{x}, q_{y}, q_{z}\right)$. The neutral surface of the two-dimensional isotropic Swift-Hohenberg equation is shown in Fig. 1.2. While here in the isotropic case the surface is radially symmetric, this would not be the case anymore under anisotropic conditions.

Let's consider the neutral curve for the isotropic case as shown on the left of Fig. 1.2. Along the neutral curve all perturbations with wave number $(q)$ are marginal stable. The curve has its minimum at $\varepsilon=0$ and $q=q_{c}$, where only patterns with wave number $q_{c}$ become unstable. For $\varepsilon>0$ a band of possible wave numbers with $\sqrt{q_{c}^{2}-\sqrt{\varepsilon}}<q<\sqrt{q_{c}^{2}+\sqrt{\varepsilon}}$ can appear. Such a neutral curve with a critical wave number $q_{c}$ at its minimum is common in a variety of pattern forming systems and therefore in many PFS certain patterns occur with a well defined wave number, which is mostly the wave number that become first unstable $\left(q_{c}\right)$.

Often, one is only interested in the relation between the amplitude of a periodic pattern and the control parameter $\varepsilon$. Considering a simple roll pattern with the critical wave number and a constant amplitude $A$ in lateral direction:

$$
\theta=A e^{\mathrm{i} q_{c} x}+\text { c.c. }
$$

One can now deduce from the SHE an equation for the amplitude of this steady stripe pattern with wave number $q=q_{c}$ by inserting Eq. 1.3 into Eq. 1.1 (see e.g. $[24,19])$ :

$$
0=\varepsilon A-A^{3} \text {. }
$$

This equation is the order parameter equation and connects the order parameter $A$ with the control parameter $\varepsilon$. It describes a pitchfork bifurcation for the amplitude $A$ at $\varepsilon=0$ (Fig. 1.3). For negative $\varepsilon$ only one stable solution with $A_{1}=0$ exist. When $\varepsilon$ exceeds zero, two other possible solutions occur:

$$
A_{2}=\sqrt{\varepsilon} \text { and } A_{3}=-\sqrt{\varepsilon} \text {. }
$$



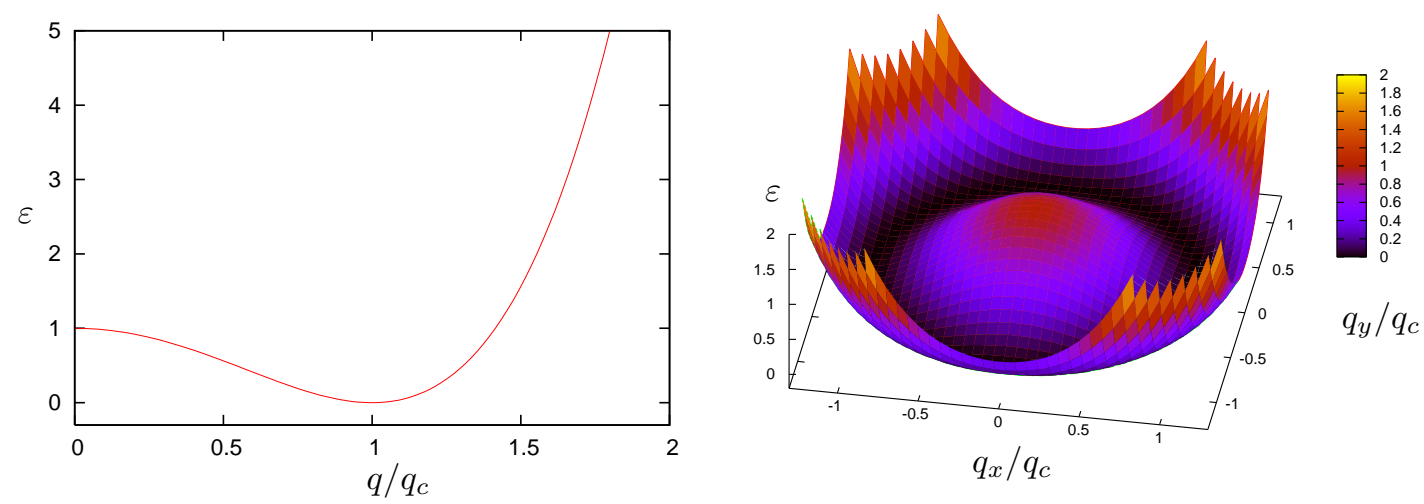

Figure 1.2: Neutral curve (left) and neutral surface (right) of rolls for the twodimensional isotropic Swift-Hohenberg equation (Eq. 1.1).

Via linear stability analysis one can show, that at the bifurcation point $\varepsilon=0$, the solution $A_{1}$ becomes unstable, while the new occurring solutions $A_{2}$ and $A_{3}$ are both stable.

This is a very simplified view. Since for $\varepsilon>0$ a band of possible wave numbers exists, the amplitude $A$ is not constant, but can vary slowly in space and time. In this case the amplitude is usually called envelope. Out of the SHE one can derive via multiple-scale perturbation analysis equations, that describe the evolution of the envelop [24]. In example, for the one dimensional case the complex amplitude $A=A(x, t)$ can be described by the Ginzburg-Landau equation with real coefficients $[25,26]$ :

$$
\frac{\partial A}{\partial T}=\varepsilon A-|A|^{2} A+\frac{\partial^{2} A}{\partial X^{2}} .
$$

Note, that in this equation $T$ and $X$ are rescaled (slowly varying) variables of time $(t)$ and space $(x)$. For very simple pattern forming systems an amplitude equation like Eq. 1.5 can be derived out of first order principles through a weakly nonlinear analysis.

Equations such as the Ginzburg-Landau equation are very helpful since they provide simplified models which nevertheless show all characteristic features of a pattern forming system. Especially the hydrodynamics of Newtonian fluids, where the governing equations are well known, is suitable to study pattern formation, since here it is often possible to derive order parameter equations similar to Eq. 1.4 and Eq. 1.5 out of first principles. The flow between two counter rotating cylinders (Taylor-Couette system) or the surface deformation of a fluid under vertical vibrations (Farraday instability) are just two examples for hydrodynamic pattern forming systems. Another archetype and probable the best studied is a 


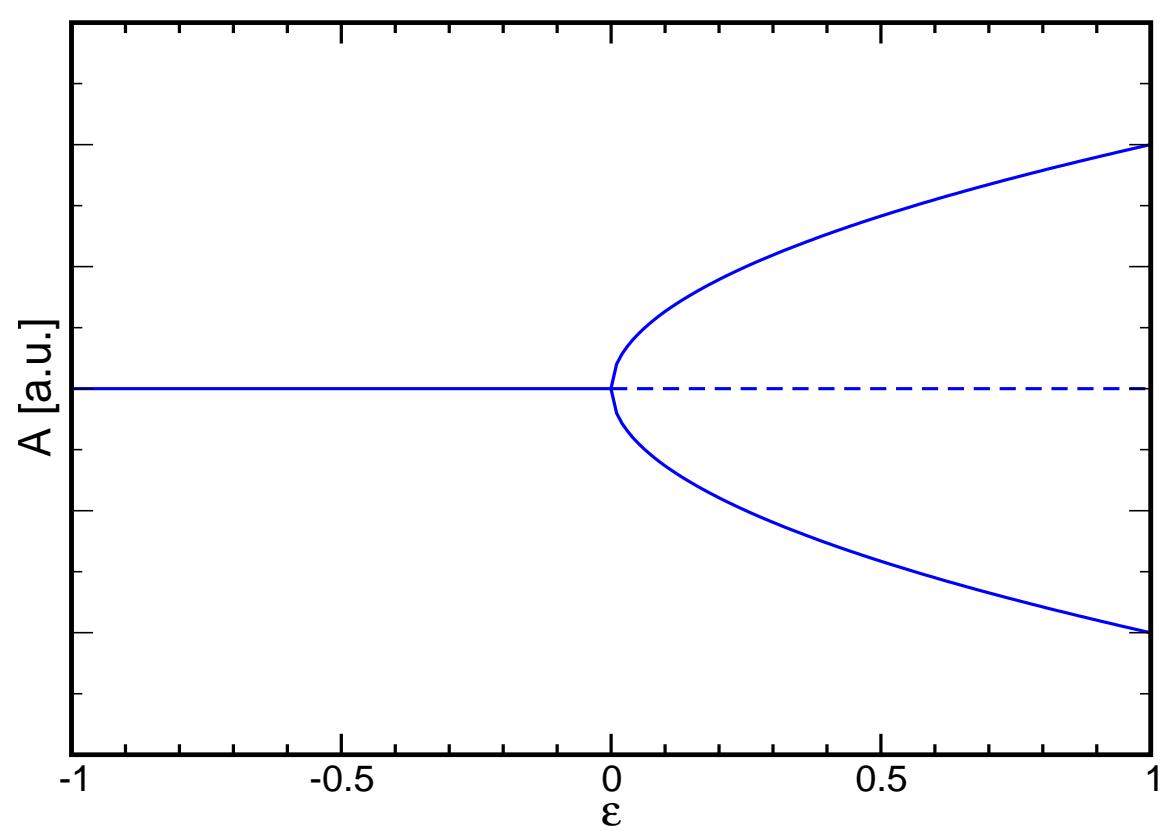

Figure 1.3: Pitchfork bifurcation. The solid line mark stable solution and the dashed line marks the unstable solution of Eq. 1.4.

fluid driven by a thermal gradient - thermal convection.

\subsection{Convection Driven by a Thermal Gradient}

Fluid motion driven by thermal convection is an important ingredient in all kinds of natural processes. For temperatures found on earth, it is the most efficient way to transport energy in fluids. It is not only the driving force for the circulation in oceans and the atmosphere, but it also causes the earth magnetic field [27, 28] and is a major force for the continental drift [29]. Beside this, thermal convection is a very convenient model system to study pattern formation [30]. It shows a large variety of different patterns like parallel rolls, hexagons, and spirals. In addition other general features of a PFS can be observed and studied in detail, such as a neutral curve with a critical wave number, several transitions between different states and their corresponding bifurcation phenomena, or a variety of instability mechanisms (e.g. Eckhaus, zig-zag, skewed-varicose) that drive the pattern to a new stable state. Here, the underlying mechanisms are well known and an order equation which describes quantitatively the onset of convection can be derived out of basic principles.

Convection occurs, where a fluid is exposed to a sufficiently large temperature gradient so that the buoyancy of a warm - low dense - fluid element is large 


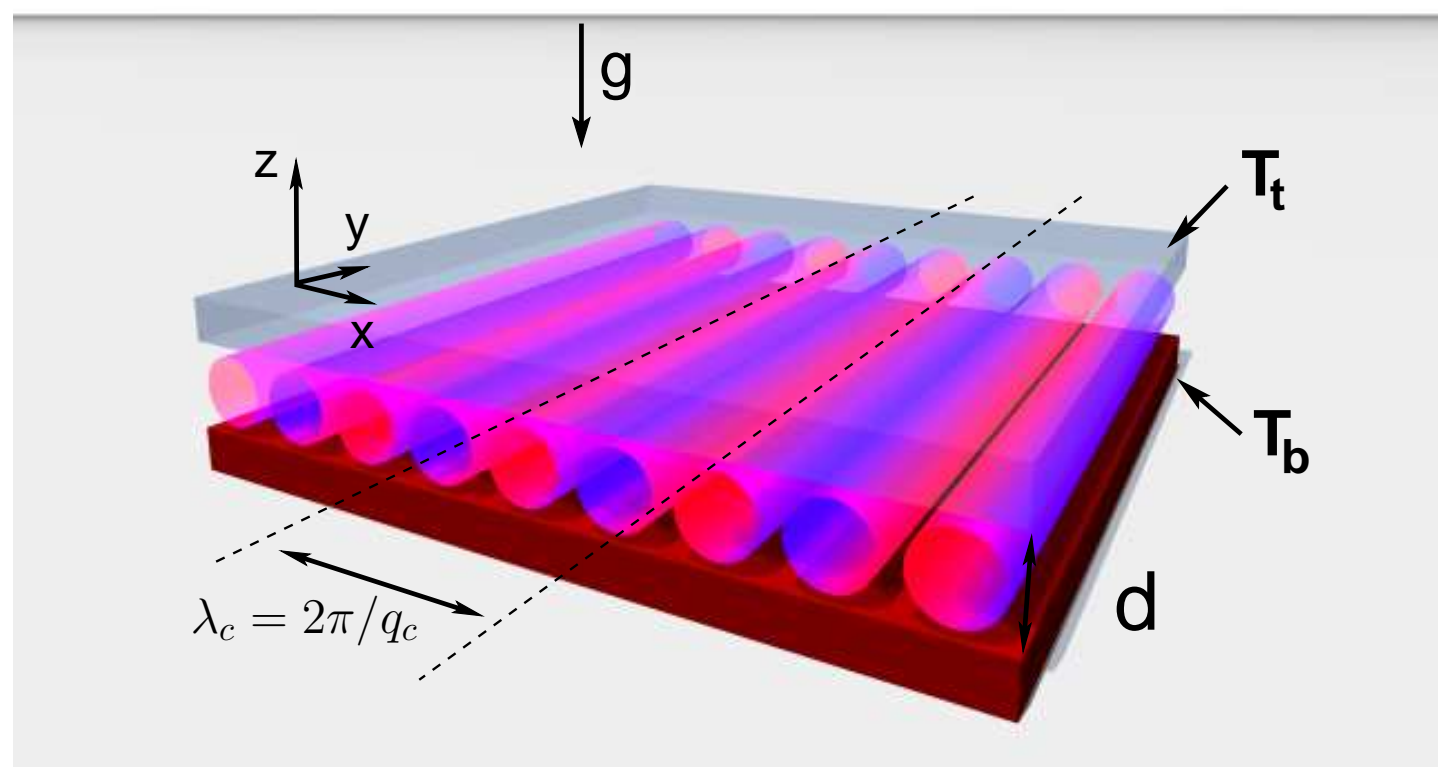

Figure 1.4: Schematic of a convection cell with periodic fluid motion.

enough to overcome the viscous drag and the damping effect of heat diffusion to its vicinity. Experimentally this can be realized by a fluid heated from below and cooled from above. While for the first serious publication on thermal convection by Henri Bénard [31] experiments were done in an open container, in later experiments the fluid was confined in vertical direction by two solid plates (see [30] for an overview of recent experiment). In that way the influence of surface tension at the free surface of the fluid is eliminated. In fact, as it turned out later, the influence of the temperature on the surface tension is so large (Marangoni effect), that Bénards convection was mainly driven by this effect and buoyancy only played a minor roll [32].

Consider a horizontal fluid layer of height $d$ between two plates of constant temperature as shown in Fig. 1.4. For small temperature differences $\Delta T=T_{b}-T_{t}$ between the confining plates, all the heat is transported by thermal conduction while the fluid is at rest. In this case small fluctuations in temperature are immediately damped by thermal diffusion while the viscosity damps fluctuations in fluid motion. If the temperature difference exceeds a critical value $\Delta T_{c}$, small perturbations are not damped anymore but amplified. The fluid starts to flow in a periodic way. As a function of the fluid properties and the boundary conditions of the system, either parallel rolls, squares or hexagons occur. This system is known as the Rayleigh-Bénard system or Rayleigh-Bénard convection (RBC). 
RBC can be described by two dimensionless parameters. The first one is the Rayleigh number:

$$
R a=\frac{\alpha g \Delta T d^{3}}{\kappa \nu}
$$

which is the ratio between the driving buoyancy and the damping mechanisms viscous drag and thermal diffusion. Here $d$ is the fluid height, $g$ the acceleration of gravity, $\alpha$ the thermal expansion coefficient and $\kappa$ and $\nu$ are thermal diffusivity and kinematic viscosity, respectively. The second parameter is the Prandtl number

$$
\operatorname{Pr}=\nu / \kappa,
$$

which is the ratio of the two damping mechanisms viscosity and heat conduction. While the Prandtl number only contains parameters which are given by the properties of the working fluid, the Rayleigh number contains the temperature difference $\Delta T$, which can easily be changed during the experiment. Therefore, $R a$ is considered as the control parameter of the system.

Via linear stability analysis one can calculate the neutral curve (see e.g. [16]) in the $q-R a$ phase diagram at which the growth rate of small perturbations with wave numbers $q$ is zero (Fig. 1.5). The minimum of this curve is at

$$
R a_{c}=1707.8 \text { and } q_{c}=3.117 / d .
$$

This means, that at $R a=R a_{c}$ convection sets in and rolls appear with a wave number of $q=q_{c}$. For increasing $R a$ a band of possible wave numbers opens.

Nonlinear analysis [33] shows the existence of a small region where straight rolls with wave number $q$ are stable. This region, the so called Busse balloon, is enclosed by various kinds of instabilities (Fig. 1.6) which are mechanisms to adjust the wave number to a value inside the Busse balloon. The shape of that region in the $q-R a$ phase space depends on $\operatorname{Pr}$ as well, and therefore one can plot the stability region in a 3-dimensional phase space spanned by $q, R a$, and $\operatorname{Pr}$.

Cross sections of the Busse balloon at $\operatorname{Pr}=0.7$ and $\operatorname{Pr}=7.0$ are shown in Fig. 1.6. For $\operatorname{Pr}=0.7$, the region of stable rolls (streaked area) is confined for large $R a$ by a skewed-varicose instability. That is a long wavelength instability which leads to a pinch off effect resulting in a pair of dislocations and a decrease of the wave number [34]. In that way the system can lower its wave number and stay in a region where straight rolls exist. On the other hand if the system shows a wave number smaller than $q_{c}$, the Busse balloon is constrained on the left side by oscillatory-, Eckhaus-, zig-zag- or crossroll instabilities which lead to an increase of the wave number [34].

While the 3-dimensional stability region for straight rolls is largely explored theoretically, most experiments where conducted in silicone oil $(\operatorname{Pr}=16 \ldots 126)$ [35, 36], water $(\operatorname{Pr}=7)[34]$, and compressed gases $(\operatorname{Pr} \approx 1)[37,30]$. 


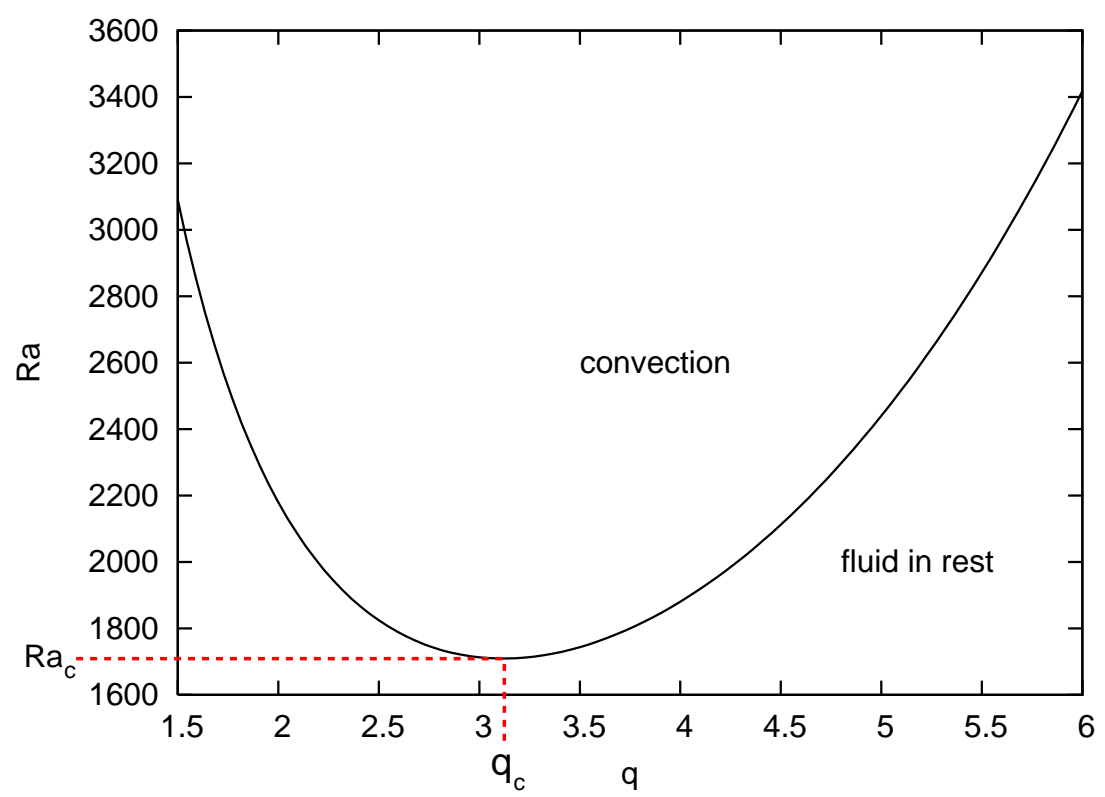

Figure 1.5: Neutral curve for Rayleigh Bénard convection
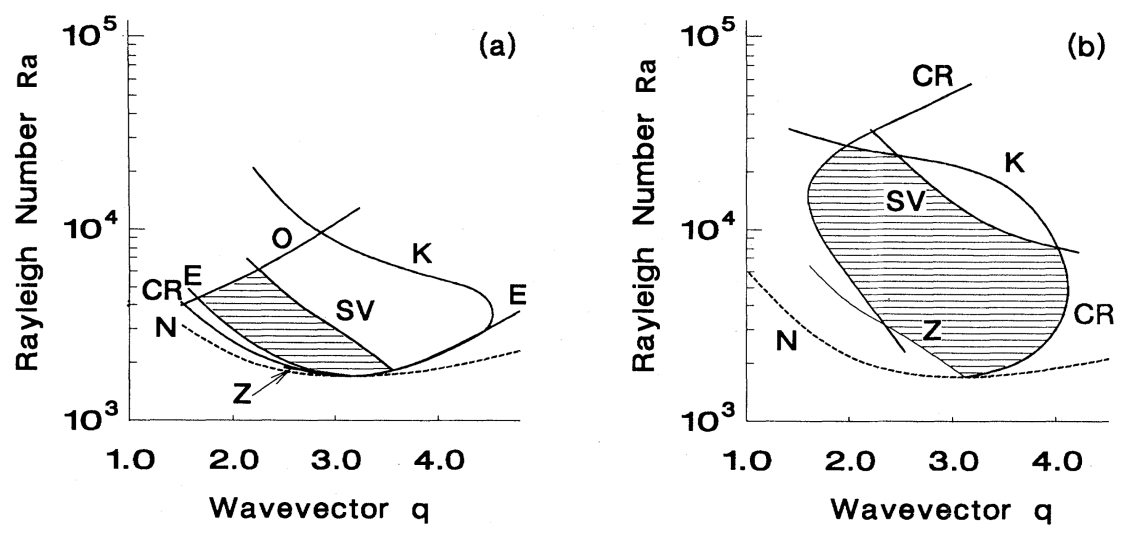

Figure 1.6: Stability regions of ideal straight rolls (streaked area), for Prandtl numbers $\operatorname{Pr}=0.71$ (left) and $\operatorname{Pr}=7.0$ (right). Dashed line, labeled with $\mathrm{N}$ shows neutral curve. Solid lines mark different instability mechanism such as: cross-rolls (CR), Eckhaus (E), zig-zag (Z), skewed-varicose (SK), knot (K), and oscillatory $(\mathrm{O})$. Figures from [15] 
Several of the occurring instability mechanisms were found theoretically by Busse, Whitehead and Clever [36, 35, 33, 34]. For an experimental verification, rolls with a specific wave number were forced via a $500 \mathrm{~W}$ incandescent lamp [38, 36, 35]. The IR radiation of the lamb was absorbed by the convective silicone oil and thus, energy was deposited inside the working fluid. By using a mask with a periodic stripe array, the energy deposition was modulated, resulting in a local heating of the water along parallel stripes. In this way, convection rolls with a well defined wave number could be forced. After switching off the light, the system either kept the forced wave number if forcing occurred within the Busse balloon or it showed one of several instabilities in order to adjust the system to a stable wave number. In this way the stability region was explored.

\subsection{Pattern Formation in Anisotropic Systems}

Pattern formation in general is associated with a spontaneous symmetry breaking. In idealized model systems, such as in RBC in large aspect ratio cells, symmetry breaking occurs under isotropic conditions. In nature, pattern formation often takes place in anisotropic systems and under the influence of non-ideal and often inhomogeneous boundaries. This might be the topography of the earth surface during cloud formation [39], the stress field during the development of fingerprints [40] or even several boundary condition during plankton growth in the sea [41]. Therefore, in the past pattern formation was studied in various anisotropic systems such as in ferrofluids [42], in chemical surface reactions [43] or on structured soil surfaces in arctic and alpine regions [44]. In all these cases an anisotropy did not only orient the occurring pattern, but also changed their morphology. An hexagonal pattern under isotropic condition often turns into a stripe like pattern if the rotational symmetry is broken ( see [44, 42, 45]).

Breaking the in-plane isotropy of the Rayleigh-Bénard system can easily be achieved by tilting the convection system. Now, the gravitational field does not act perpendicular to the confining plates anymore, but has also a component parallel to the convection plane. In the first experiments on inclined layer convection (ILC) by U. Kurzweg [46], it was shown that the critical temperature difference for the transition from conduction to convection, increases with increasing inclination angle $\gamma$ by $\Delta T_{c}(\gamma)=\Delta T_{0} / \cos (\gamma)$, whereas $\Delta T_{0}$ is the critical temperature difference in the non-inclined case. This increase in $\Delta T_{c}$ is due to the reduced effective gravitational acceleration by exactly the factor $\cos (\gamma)$, resulting in a reduced buoyancy of warm fluid elements (Fig. 1.7).

Further experiments and theoretical considerations have been done on ILC in recent years $[47,48,49,50,51]$. Besides an increase in the critical temperature difference, it was found for inclination angles smaller than a critical angle $\left(\gamma_{c d}\right)$, that the occurring parallel rolls at convection onset are aligned parallel to the 
(a)
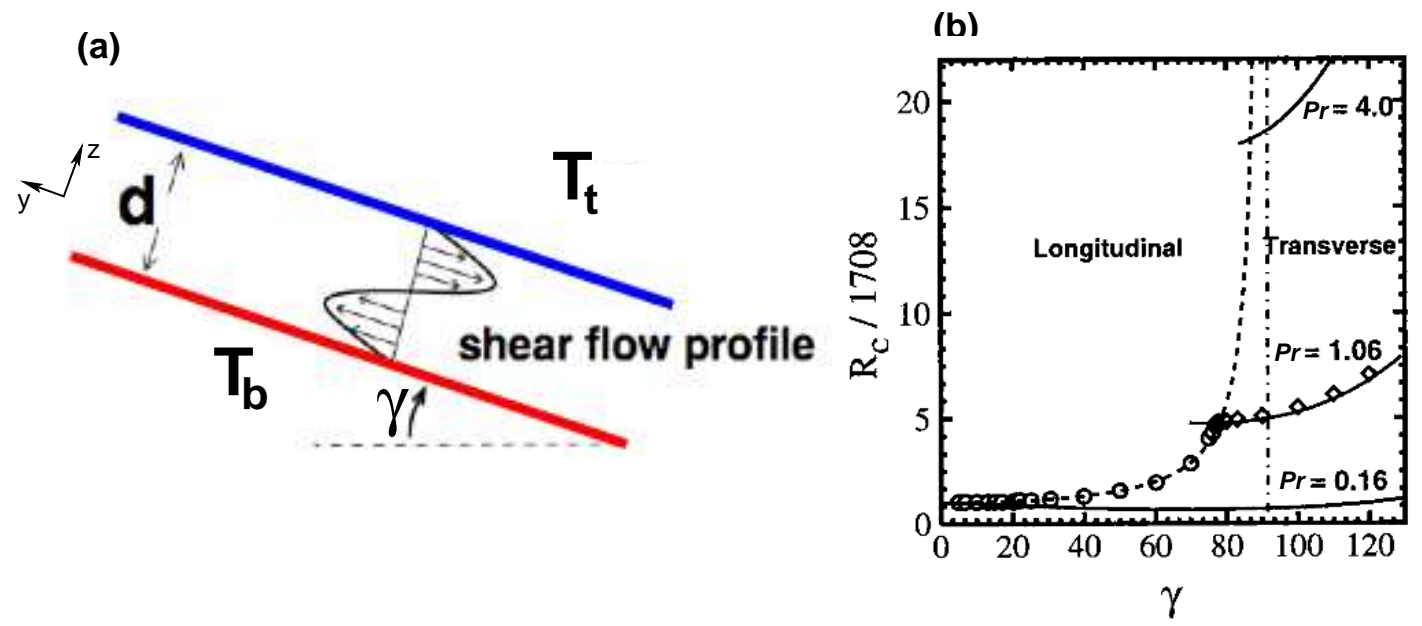

Figure 1.7: Inclined layer convection (ILC). (a): Due to inclination a cubic shear flow occurs which breaks the symmetry and aligns the occurring convection rolls parallel to gravity for $\gamma<\gamma_{c d}$. (b): Onset of convection in dependence of the inclination angle $\gamma$ for various $\operatorname{Pr}$ for longitudinal rolls (dashed) and transverse rolls (solid). The dotdashed line marks inclination angles of $90^{\circ}$. The points are experimental data, taken in compressed gas at $\operatorname{Pr}=1.06$ (figure from [30]).

in-plane gravity component, termed longitudinal rolls. This is due to a large scale shear flow which transports warm fluid along the warm plate in uphill direction and cold fluid along the cold plate in downhill direction (see Fig. 1.7a). A critical angle $\gamma_{c d}$, which depends on the Prandtl number $\operatorname{Pr}$ exists, at which the situation changes. For $\gamma>\gamma_{c d}$ the onset of convection rolls is no longer $\propto 1 / \cos (\gamma)$ but less steep. In addition, the occurring rolls are aligned with their axis perpendicular to gravity and drift slowly in downhill direction (transverse rolls). Note, that the experimentally observed drift was not found in simulations and may be attributed to inhomogeneous fluid properties (non-Boussinesq effects). Transverse rolls are no longer driven by a thermal convection instability but rather by a shear instability of the Kelvin- Helmholtz type [52] and therefore even appear for $\gamma>90^{\circ}$. Due to the shear flow new kinds of steady and dynamical pattern where found for larger Rayleigh numbers, such as ordered and chaotic undulations (described in detail in [50]), subharmonic oscillations, transverse bursts, or longitudinal bursts $[53,48]$. 


\subsection{Temporal and Spatial Periodic Forcing of Pattern Forming Systems}

If one speaks about forcing in the context of pattern forming systems one mostly means that a parameter is modulated periodically in time (temporal), space (spatial) or both (spatio-temporal). Temporal forcing was studied extensively in a variety of non-linear dynamical systems in both theory and experiments (see e.g. $[54,55])$ where it became interesting for engineers in order to control chaotic behavior of non-linear systems [56]. Temporal forcing was also applied to quasi two-dimensional and three-dimensional pattern forming systems. For example, Petrov, Ouyang and Swinney applied time periodic forcing on the BelousovZhabotinsky reaction [57]. They used light pulses to force the light sensitive chemical reaction and observed different frequency-locked pattern, and standingwave pattern as a function of the forcing frequency. The effect of time periodic forcing in three-dimensional excitable media such as in example in heart tissue, was investigated in numerical simulations by Alonso, Sagués and Mikhailov [58]. It was found that also here a spatio-temporal chaotic state could be suppressed by applying a time period forcing. Applications of this knowledge in cardiology was suggested, were certain types of ventricular fibrillation could be terminated with this approach, that would otherwise lead to cardiac death.

Time periodic forcing was also applied on Rayleigh-Bénard convection. In first experiments in the group of G. Ahlers, a temporal periodic modulation of the bottom plate temperature was applied, while the temperature of the top plate was held constant [59]. As predicted in theory (e.g. in [60]), it was found that due to the modulation the conduction state was stabilized and the onset occurred at higher Rayleigh numbers than in the unmodulated case. Furthermore, hexagons were observed at convection onset instead of straight rolls. This was attributed to the broken up-down symmetry of the system by modulating only the temperature at the bottom plate.

Later, the influence of a periodic modulation of the acceleration of gravity was studied in theory and in experiments by two groups in Atlanta and in Bayreuth $[61,62,63]$. One of the main results was the occurrence of patterns with harmonic and subharmonic wave numbers that resulted in superlattice patterns. It was also found that in this configuration, convection also occurs when the top plate is warmer then the bottom plate.

In temporal forcing, the system has the ability to respond with resonances in time. While the forcing of a quasi one-dimensional system is quite similar to that of the time periodic case, in spatial periodic forcing of a quasi two-dimensional system, the system, has a second dimension were it can build resonances. A forcing in only one direction can easily lead to a more complex two-dimensional pattern when in example a mode in the pattern resonates with the forcing mode 
as will be shown several times in this thesis.

As mentioned earlier, in thermal convection spatial forcing was used in experiments by Whitehead, Chen and Busse [38, 36, 35] in order to check the stability of rolls with specific wave numbers. Later on in the 1980s Lowe, Gollub and Lubensky [64, 65] conducted comprehensive experiments on spatial forcing in electroconvection in a thin layer of nematic liquid crystal. There, the forcing was done by a photolithographically fabricated lower electrode. In this way two interdigitated regions with different potentials were created. The ratio of the applied forcing wave number $q_{f}$ and the critical wave number of the systems $q_{c}$ could be varied by changing the thickness of the liquid crystal layer. The result was a rich variety of different new phenomena, like commensurate patterns where the structural periodicity is rationally related to the forced one, or incommensurate patterns consisting of arrays of kink defects or solitons.

In light of the experimental results by Lowe, Gollub and Lubensky [64, 65], Coullet [66] derived an amplitude equation for a one-dimensional periodic pattern under the influence of spatial periodic forcing. Based on Eq. 1.5 he used symmetry arguments and showed that in case of a rational ratio between the forced and the intrinsic wave number $\left(m \cdot q_{f}=n \cdot q_{c}, \quad m, n \in \mathbb{N}\right)$ an additional term has to be added to the one-dimensional amplitude equation:

$$
\frac{\partial A}{\partial T}=\varepsilon A-|A|^{2} A+\delta\left(A^{*}\right)^{n-1}+\frac{\partial^{2} A}{\partial X^{2}} .
$$

Here again, $\varepsilon$ is the control parameter, $T$ and $X$ are scaled (slowly varying) time and space and $\delta$ is the forcing parameter that denotes the forcing strength.

He then considered spatially homogeneous steady state solutions $\left(\frac{\partial A}{\partial t}=0\right.$ and $\left.\frac{\partial^{2} A}{\partial x^{2}}=0\right)$. It turned out that $n$ determines the type of bifurcation that $A$ undergoes as a function of $\varepsilon$. In the resonant case $(n=1)$ the bifurcation is imperfect which means that even for negative $\varepsilon$ the amplitude $A$ never becomes zero (red curve in Fig. 1.8). For $n=2$ the bifurcation is perfect, but shifted to smaller $\varepsilon$, and for $n=3$ the bifurcation becomes transcritical.

Spatially forcing in the Rayleigh-Bénard system was considered theoretically in 1977 by Kelly and Pal [67], who assumed modulated vertical boundaries and derived analytically an amplitude equation very similar to Eq. 1.8. Recently McCoy [68] verified their approach experimentally. He used a quasi one-dimensional surface corrugation of the bottom plate in order to break the translational symmetry of the system. By choosing a forcing wave number close to the critical wave number of the system he could not only show the occurrence of an imperfect bifurcation at onset, but in addition he found for higher $R a$ phase kinks as an instability of the forced rolls [69]. These kinks show remarkable dynamics such as annihilation and formation of large stable clusters. 


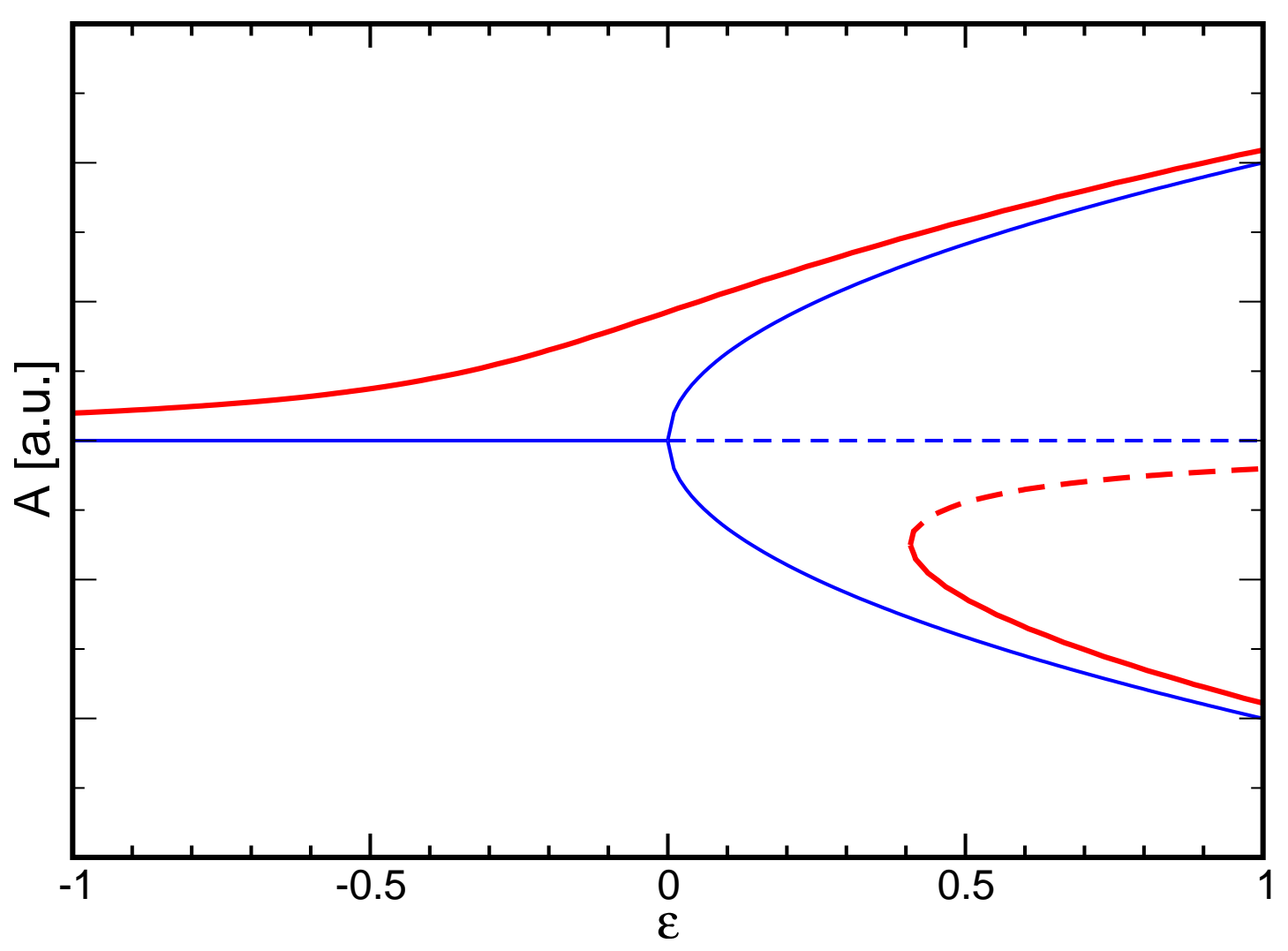

Figure 1.8: Pitchfork bifurcation (blue) and imperfect bifurcation (red) as steady solutions of Eq. 1.8. The solid lines mark the stable solutions and the dashed lines mark unstable solutions. The pitchfork bifurcation is a solution with $\delta=0$ and the imperfect bifurcation is a solution with $\delta>0$ and $n=1$.

The present work is a direct continuation of McCoys work. Here, the influence of different symmetry breaking mechanisms on the pattern forming Rayleigh-Bénard system is studied. In addition to a periodic spatial forcing, also the rotational symmetry is broken by tilting the convection cell. These two different mechanisms can either act together to stabilize the appearing pattern or compete with each other and create new unexpected phenomena, such as hexagonal resonances or superlattice patterns. The relative strength of these mechanisms can be tuned carefully by changing the inclination angle, their relative orientation and the temperature difference. In this way new patterns are observed and instability mechanisms explored.

The following two chapters give a theoretical background (Chapter 2) and explain the experimental setup (Chapter 3). Chapter 4 shows experimental results of boundary induced resonant forcing in an inclined layer convection cell. In Chapter 5 experiments are presented, where the forcing wave number $q_{f}$ was varied in a 
horizontal thermal convection system (standard RBC). The occurring patterns and their stabilities are investigated. In Chapter 5 an outlook is given to future experiments on optical forcing. The thesis ends with a short summary. 


\section{Chapter 2}

\section{Theoretical Overview}

\subsection{Boussinesq Equations}

A fluid, confined in vertical direction by two infinitely conducting plates with temperatures $T_{b}$ and $T_{t}$, is considered (see Fig. 1.4). The plates extend to infinity in lateral direction and are located at positions $z=-d / 2$ and $z=d / 2$. The velocity field $\vec{v}(\vec{r}, t)$, the temperature field $T(\vec{r}, t)$, the pressure $P(\vec{r}, t)$, and the mass density $\varrho(\vec{r}, t)$ are coupled by the following three equations:

- The continuity equation (mass conservation):

$$
\frac{\partial \varrho}{\partial t}+\nabla(\varrho \vec{v})=0
$$

- The Navier-Stokes equation (momentum conservation):

$$
\left(\frac{\partial \vec{v}}{\partial t}+(\vec{v} \cdot \nabla) \vec{v}\right)=-\frac{\nabla P}{\varrho}+\nu \nabla^{2} \vec{v}-g \hat{z}
$$

- The heat equation (energy conservation):

$$
\frac{\partial T}{\partial t}+(\vec{v} \cdot \nabla) T=\kappa \nabla^{2} T
$$

The parameters that occur in these equations are the gravitational acceleration $g$, the kinematic viscosity $\nu$, and the thermal diffusivity $\kappa$. The unit vector in zdirection is denoted by $\hat{z}$. In general, the material parameters $(\varrho, \nu, \kappa)$ depend on 
the temperature and the pressure. For very small temperature differences between the top and the bottom plate and for small cell heights $d$, these parameters can be assumed to be constant. Solely in the buoyancy term of the Navier-Stokes equation, one assumes a linear temperature dependency of the density:

$$
\varrho=\varrho_{0}\left(1-\alpha\left(T-T_{0}\right)\right)
$$

Here, $\varrho_{0}$ denotes the density at the average temperature $T_{0}=\left(T_{b}+T_{t}\right) / 2$ and $\alpha$ is the thermal expansion coefficient. This approximation was suggested by Boussinesq [70] and is the roughest possible approximation. In addition, one assumes an incompressible fluid, which seems to be paradox on the first glance. But it was shown by Spiegel and Veronis [71] that the approximation works well if the temperature difference and the cell height are sufficiently small.

The easiest solution is the conduction solution where the fluid is in rest $(\vec{v}=0)$ and the temperature field is given by a linear profile:

$$
T_{\text {cond }}(z)=T_{0}-\frac{z}{d} \Delta T \quad ;-d / 2 \leq z \leq d / 2 .
$$

One now assumes small deviations from the conduction solution for the temperature $\left(T=T_{\text {cond }}(z)+\theta(\vec{r}, t)\right)$, the pressure $\left(P=P_{0}(z)+p(\vec{r}, t)\right)$ and for the velocity $v(\vec{r}, t)$.

In order to compare different terms in the differential equations, one introduces dimensionless variables. Length is expressed in units of $d$, time in units of the vertical heat diffusion time $t_{\kappa}=d^{2} / \kappa$, the pressure in units of $\varrho_{0} \kappa \nu / d^{2}$, and the temperature in units of $\nu \kappa / \alpha g d^{3}$

With these approximations, Eq. 2.1, 2.2 and 2.3 can be written as:

$$
\begin{aligned}
& \nabla \vec{v}=0, \\
& \frac{1}{\operatorname{Pr}}\left(\frac{\partial \vec{v}}{\partial t}+(\vec{v} \cdot \nabla) \vec{v}\right)=-\nabla p+\nabla^{2} \vec{v}+\theta \hat{z}, \\
& \left(\frac{\partial \theta}{\partial t}+(\vec{v} \cdot \nabla) \theta\right)=\nabla^{2} \theta+R a(\vec{v} \hat{z}) .
\end{aligned}
$$

Note, that often the pressure is expressed in units of $\varrho \kappa^{2} / d$ which leads to $\operatorname{Pr}$ as a factor in front of the $\nabla p$-term (e.g. in [16]). On the first glance this seems to make an important change for large $\mathrm{Pr}$. In fact, the pressure term disappears by applying $\nabla \times$ to the equations later on. 
This set of equations is known as the Boussinesq equations, in which all system parameters are collected in the dimensionless $R a$ and Pr, which are Rayleigh and Prandtl number as defined in Eq. 1.6 and 1.7.

For small perturbations from the conduction state $(v \ll 1$ and $\theta \ll 1)$ one can calculate via linear stability analysis whether the conduction state is stable, (small perturbations are damped) or whether it is unstable (perturbation amplitude grows exponentially). In this way one gets the neutral curve as shown in Fig. 1.5 and discussed in the previous chapter. For $\varepsilon$ under the neutral curve, small fluctuations are damped. If a fluid element is slightly warmer than its surrounding environment, and starts to move due to its buoyancy, its motion will be damped by viscous drag, and it will lose all its heat to its vicinity, before it can reach higher (colder) fluid layers. For larger $R a$ (areas above the neutral curve in Fig. 1.5), any small fluctuation in temperature, fluid velocity, or pressure is amplified and fluid motion (convection) sets in. In case of ridged no-slip boundaries in vertical direction, the neutral curve has a minimum at

$$
q_{c}=3.117 \quad \text { and } \quad R a_{c}=1707.8 \quad \text { (see e.g. [16]). }
$$

Therefore, if one increases $R a$ slowly from values smaller to values larger than $R a_{c}$ straight rolls with wavelength $\lambda_{c}=\frac{2 \pi}{q_{c}} d \approx 2 d$ occur at $R a_{c}$.

In isotropic convection systems the neutral curve does only depend on the wave number $q$ and not on the orientation of the wave vector. The orientation of convection rolls at their onset is random in this ideal case but in real experiments often influenced by the sidewalls (see also Fig. 4.4 and [30]).

\subsection{Surface Corrugation}

A theoretical description for a RBC system, which is forced by periodically modulated boundaries was developed by Kelly and Pal [67], Pesch [72] and McCoy [68]. For simplicity one assumes a bottom plate, whose surface is sinusoidally modulated with a wave number $q_{f} .{ }^{1}$ The amplitude of the one-dimensional surface profile is $\delta$ and the boundaries have constant temperature:

$$
\left.T(x, y, z)\right|_{z=d\left[-\frac{1}{2}+\delta \cos \left(q_{f} x\right)\right]}=T_{b}
$$

It is shown in [67] and [68] that at $R a$ close to $R a_{c}$, a surface corrugation of the lower boundary is equivalent to a periodic temperature modulation of a flat

\footnotetext{
${ }^{1}$ One should note, that experimentally it is difficult to produce a sinusoidal surface and hence, for the experiments described in the next chapters a bottom plate with a rectangular surface profile was used. For quantitative comparison between experiment and theory, the rectangular profile of the bottom plate surface was approximated by its first Fourier mode.
} 
surface. In this case the boundary conditions can be written as:

$$
\begin{aligned}
\left.T(x, y, z)\right|_{z=-d / 2} & =T_{b}\left(1+\delta \cos \left(q_{f} x\right)\right) \\
\left.T(x, y, z)\right|_{z=d / 2} & =T_{t} .
\end{aligned}
$$

A temperature profile, which fulfills the boundary condition and the steady heat equation, is given by a linear profile plus a term, due to the modulation:

$$
\begin{aligned}
T_{\text {cond }}(x, y, z) & =T_{0}+\Delta T \frac{z}{d}+\delta T_{b} \frac{\sinh \left[(d / 2-z) q_{f}\right]}{\sinh \left(q_{f} d\right)} \cos \left(q_{f} x\right) \\
& =T_{\text {lin }}+\delta T_{\text {mod }} .
\end{aligned}
$$

One should mention that $T_{\text {cond }}$ solves the steady heat equation (Laplace equation) but is not a solution for the steady momentum equation (Eq. 2.2) ${ }^{2}$. Nevertheless the new $T_{\text {cond }}$ is very helpful, since now one can write down the temperature field in a similar way to the unforced system:

$$
T(x, y, z)=T_{\text {lin }}(z)+\delta T_{\text {mod }}(x, z)+\theta(x, y, z)=T_{\text {cond }}+\theta,
$$

where $\theta(\vec{r})=0$ at $z= \pm d / 2$.

Due to the incompressibility condition (Eq. 2.6), the velocity field can be decomposed into a toroidal and a poloidal velocity field as :

$$
\vec{v}=\vec{v}_{t}+\vec{v}_{p}
$$

Whereas to each field one can find potentials $f$ and $g$ such that:

$$
\begin{aligned}
\vec{v}_{t} & =\nabla \times g \hat{z}=\hat{\epsilon} g, \\
\vec{v}_{p} & =\nabla \times(\nabla \times f \hat{z})=\hat{\delta} f .
\end{aligned}
$$

Here, following new differential operators were introduced:

$$
\begin{aligned}
\hat{\epsilon} & =\left(\begin{array}{c}
\partial_{y} \\
-\partial_{x} \\
0
\end{array}\right) \text { and } \\
\hat{\delta} & =\left(\begin{array}{c}
\partial_{z} \partial_{x} \\
\partial_{z} \partial_{y} \\
-\left(\partial_{x}^{2}+\partial_{y}^{2}\right)
\end{array}\right)=\left(\begin{array}{c}
\partial_{z} \partial_{x} \\
\partial_{z} \partial_{y} \\
\Delta_{2}
\end{array}\right) .
\end{aligned}
$$

\footnotetext{
${ }^{2} \mathrm{~A}$ pure conduction state cannot exist for any $\Delta T \neq 0$
} 
Up to here, every step is equivalent to the non-modulated thermal convection case. Now, one introduces the temperature modulation into the Boussinesq equations (Eq. 2.6-2.8), simply by substituting every occurrence of $\theta$ by:

$$
\theta+\delta \frac{R a}{\Delta T} T_{\text {mod }}
$$

By inserting the velocity potentials in Eq. 2.6-2.8 and applying $\hat{\delta}$ and $\hat{\epsilon}$ from the left, one gets rid of the pressure term and obtains three equations for the fields $\theta, g$ and $f$ :

$$
\begin{aligned}
& \partial_{t} \theta+\frac{\delta R a}{\Delta T}((\hat{\delta} f+\hat{\epsilon} g) \cdot \nabla) T_{\text {mod }}+((\hat{\delta} f+\hat{\epsilon} g) \cdot \nabla) \theta= \\
& R a \Delta_{2} f+\nabla^{2} \theta \\
& \operatorname{Pr}^{-1}\left[\nabla^{2} \Delta_{2} \partial_{t} f+\hat{\delta} \cdot((\hat{\delta} f+\hat{\epsilon} g) \cdot \nabla)(\hat{\delta} f+\hat{\epsilon} g)\right]= \\
& -\frac{\delta R a}{\Delta T} \Delta_{2} T_{m o d}-\Delta_{2} \theta+\nabla^{4} \Delta_{2} f, \\
& \operatorname{Pr}^{-1}\left[\Delta_{2} \partial_{t} g+\hat{\epsilon} \cdot((\hat{\delta} f+\hat{\epsilon} g) \cdot \nabla)(\hat{\delta} f+\hat{\epsilon} g)\right]=\nabla^{2} \Delta_{2} g \text {. }
\end{aligned}
$$

An analytical solution of these equations is very difficult to calculate. Instead, a Galerkin approach is usually used to solve the system numerically (see [51]).

For small forcing parameter $(\delta \ll 1)$ an amplitude equation can be derived for the convection strength of one-dimensional roll solutions via a perturbation approach (see $[67,68]$ ). For a one-dimensional problem one can set $g=0$ and thus, write the velocity as:

$$
\vec{v}=\left(\begin{array}{c}
\frac{\partial}{\partial z} \frac{\partial f}{\partial x} \\
\frac{\partial f}{\partial z} \\
-\frac{\partial^{2} f}{x}
\end{array}\right)=\hat{\delta} f
$$

In addition, one expands the fluid parameters in powers of a small parameter $\mu$ :

$$
\begin{aligned}
& f(x, z)=\mu f_{1}(x, z)+\mu^{2} f_{2}(x, z)+\mu^{3} f_{3}(x, z)+\ldots \\
& \theta(x, z)=\mu \theta_{1}(x, z)+\mu^{2} \theta_{2}(x, z)+\mu^{3} \theta_{3}(x, z)+\ldots
\end{aligned}
$$

One also expands $R a$ around a $R a_{0}\left(q_{f}\right)$, which is the Rayleigh number on the neutral curve for $q=q_{f}$ :

$$
R a=R a_{0}+\mu^{2} R a_{2}+\ldots
$$

Motivated by a comparison between the cubic and the constant term in the expected amplitude equation, a scaling law $\mu=\delta^{1 / 3}$ is chosen [68]. 
Note, that every expansion is done at a fixed value of $q_{f}$. Roll solutions with wave vector $q_{f}$ can be written in general by:

$$
\left(\begin{array}{c}
g \\
\theta
\end{array}\right)=\left[A \exp \left(\mathrm{i} q_{f} x\right)+c . c\right]\left(\begin{array}{c}
g_{z}(z) \\
\theta_{z}(z)
\end{array}\right) .
$$

With this ansatz, one can calculate an equation for the amplitude $A$, close to $R a_{c}$ (critical Rayleigh number at the unforced convection) for small forcing parameter $(\delta \ll 1)$ and for $q_{f} \approx q_{c}[68]$ :

$$
\frac{R a-R a_{0}\left(q_{f}\right)}{R a_{c}} A-g_{0}(P r) A|A|^{2}+g_{2}\left(q_{f}\right) \delta=0
$$

Here, $g_{0}$ and $g_{2}$ are constants that depend on $\operatorname{Pr}$ and $q_{f}$ (defined in Eq. 4.62 and 4.63 of [68]). For $q_{f} \approx q_{c}$ and a normalization as in [68], the first constant can be written as:

$$
g_{0}(\operatorname{Pr})=4.474 \cdot 10^{-6}-\frac{3.016 \cdot 10^{-8}}{\operatorname{Pr}}+\frac{5.324 \cdot 10^{-8}}{\operatorname{Pr}^{2}} \quad[68] .
$$

The second parameter $g_{2}$ depends on the exact forcing wave number. It can be calculated as shown in [68] (Eq. 4.61 and Eq. 4.62) and is for the experiments presented in the following chapter:

$$
g_{2}=238
$$

The amplitude equation for forced thermal convection describes an imperfect bifurcation, where no sharp onset of convection occurs. Instead, the convection amplitude increases continuously with increasing $R a$. Eq. 2.25 is in principal the same as Eq. 1.8 with $n=1$. While Eq. 2.25 was derived via weakly nonlinear analysis, Eq. 1.8 was derived by simple symmetry considerations out of the Ginzburg-Landau equation [66].

\subsection{Inclined Layer Convection (ILC)}

Initially, unforced inclined layer convection is considered. Boundary forcing is introduced later in this section analog to what was done above. A detailed theoretical description of unforced ILC can be found in [47] and [51]. A very short overview is given here.

The co-ordinate system is chosen as shown in Fig. 2.1. The z-direction is vertical to the convection plane and the $\mathrm{x}$ - and the $\mathrm{y}$-direction are parallel to it. Furthermore the y-axis is chosen to be parallel to the in-plane gravity component 
$\left(\vec{g}_{\|}\right)$. It will be useful to define a variable $\varphi$ as the angle between the y-axis and an arbitrary vector $\vec{r}$ as shown in Fig. 2.1b. This vector will not have any other meaning, than showing the direction of the axis of convection rolls, or the direction of the forcing structure of the bottom plate later on.

(a)

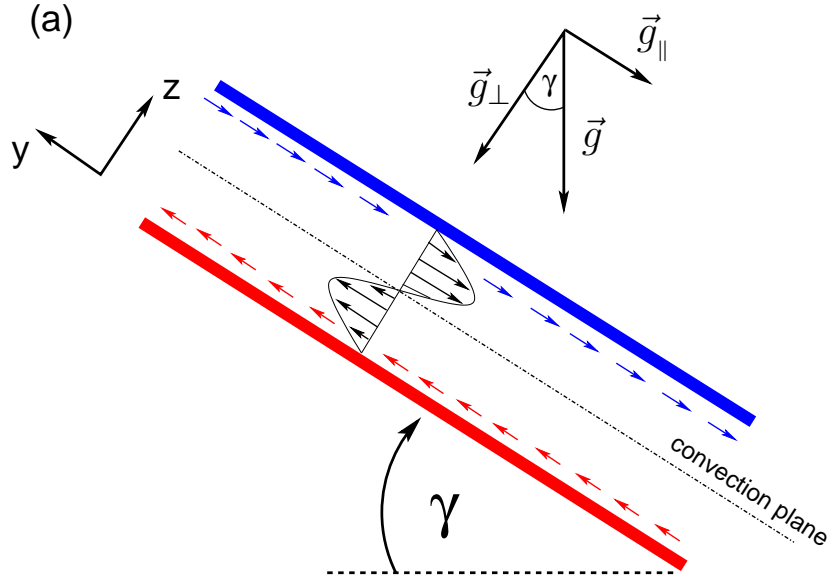

(b)

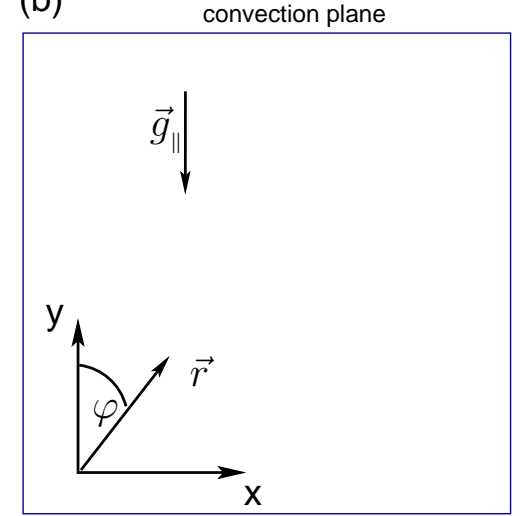

Figure 2.1: Side view (a) and topview (b) of an inclined convection cell with inclination angle $\gamma . \vec{r}$ is a vector that is parallel to the convection roll axis of a straight roll pattern.

With this definition of the co-ordinate system, the gravity has now a component in z-direction and another component in y-direction:

$$
\vec{g}=-g(\hat{z} \cos (\gamma)+\hat{y} \sin (\gamma))=\vec{g}_{\perp}+\vec{g}_{\|} .
$$

Here, only $\vec{g}_{\perp}$ contributes to a buoyancy in the z-direction and therefore a redefinition of the Rayleigh number for inclined layer convection is convenient:

$$
R a^{I}=\frac{\alpha g \cos (\gamma) \Delta T d^{3}}{\nu \kappa}=R a \cos (\gamma)
$$

For any finite temperature difference $\Delta T, R a^{I}$ becomes zero for $\gamma \rightarrow 90^{\circ}$. As a result, no buoyancy driven instability occurs anymore even for very high $\Delta T$.

Taken into consideration, that also the dimensionless temperature scale is related to the new Rayleigh number $\left(\theta \rightarrow \theta \cdot \Delta T / R a^{I}\right)$ the Boussinesq equations become (see e.g. [47] and [51]):

$$
\begin{aligned}
\operatorname{Pr}^{-1}\left(\frac{\partial \vec{v}}{\partial t}+(\vec{v} \cdot \nabla) \vec{v}\right) & =-\nabla p+\nabla^{2} \vec{v}+\theta(\hat{z}+\hat{y} \tan (\gamma)), \\
\frac{\partial \theta}{\partial t}+(\vec{v} \cdot \nabla) \theta & =R a^{I} \vec{v} \hat{z}+\nabla^{2} \theta \\
\nabla \vec{v} & =0 .
\end{aligned}
$$


A steady solution of these equations consists of a conduction profile for $T$ and a flow in y-direction:

$$
\begin{aligned}
T_{\text {cond }} & =\frac{T_{0} R a^{I}}{\Delta T}-R a^{I} \cdot z \\
\vec{U}_{0}(z) & =R a^{I} \tan (\gamma) \frac{1}{6}\left(z^{3}-\frac{1}{4} z\right) \hat{y} .
\end{aligned}
$$

The stationary base flow $U_{0}$ exists for all finite inclination angles when $R a^{I} \neq$ 0 . Warm fluid rises along the warm plate and sinks along the cold plate (see Fig. 2.1a), whereas the flow velocity of $\vec{U}_{0}$ has a cubic dependency on $z$.

It is now convenient to split the velocity into three parts [47]:

$$
\vec{v}=\vec{U}_{0}+\vec{U}_{1}+\vec{u}
$$

with $\vec{u}$ being the fluctuating component of the velocity, which fulfills the condition $\langle u\rangle_{x, y}=0$. The third quantity $U_{1}$ denotes the z-dependent modification of the basic shear flow due to the onset of convection. Out of symmetry one can assume $\vec{U}_{1}=U_{1} \hat{y}$.

For calculations of the temperature field $\theta$ and the velocity field $\vec{u}$, one again writes $\vec{u}$ as a sum of a toroidal and a poloidal field:

$$
\vec{u}=\hat{\delta} f+\hat{\epsilon} g
$$

The fields are then included into the Boussinesq- equations and one gets four differential equations for $\theta, g, f$, and $\vec{U}_{1}$ (see Appendix A.1) which can be solved numerically.

For sufficiently small $R a^{I}$, the fluctuating velocity $\vec{u}$ is zero and only the large scale shear flow $\vec{U}_{0}$ exists. In first order, the inclination does only affect the large scale shear flow $\vec{U}_{0}$ and does otherwise not occur in the equations for $\vec{u}$. Therefore, the critical Rayleigh number $\left(R a_{c}^{I}\right)$, at which $\vec{u}$ becomes finite (convection onset), has the same value then in the horizontal convection case:

$$
R a_{c}^{I}=1707.8
$$

In addition, the rolls occurring at $R a_{c}^{I}=1707.8$ have the same wave number of $q_{c}=1707.8 / d$ as in the horizontal case. This means of course, that the temperature difference, which is necessary to reach $R a_{c}^{I}$, increases with increasing inclination angle as $\Delta T_{c} \propto 1 / \cos (\gamma)$ (see also Fig. 2.2).

The large scale shear flow $\vec{U}_{0}$ breaks the symmetry of the system and leads to rolls aligned with their axis parallel to $\vec{g}_{\|}$(longitudinal rolls). Here, one can make use of the previously defined angle $\varphi$ (see Fig. 2.1), which should be from now 
on the angle between the axis of convection rolls and the y-axis (or the in-plane gravity component $\vec{g}_{\|}$). For longitudinal rolls, this angle would be $\varphi=0^{\circ}$.

Beside convection driven by buoyancy another instability can produce a finite fluctuating component $\vec{u}$. If the large scale shear flow $\vec{U}_{0}$ becomes large enough, the shear stress between the warm rising fluid at the warm plate and the cold sinking fluid at the cold plate is so strong, that the interface between cold and warm fluid becomes unstable with respect to a Kelvin-Helmholtz instability. While for small inclination angles $\gamma<\gamma_{c d}$ the shear instability occurs for $R a^{I}>1707.8$, for $\gamma>\gamma_{c d}$ the shear instability sets in even before the buoyancy driven instability $\left(R a^{I}<1707.8\right)$. Instead of buoyancy driven longitudinal rolls for $\gamma<\gamma_{c d}$, transverse rolls occur for $\gamma>\gamma_{c d}$ due to the shear instability with an angle between their axis and the y-direction of $\varphi=90^{\circ}$. As already discussed in the introduction (Sec. 1.4) and shown in Fig. 1.7b, the exact value of $\gamma_{c d}$ is a function of $\operatorname{Pr}$ and is very small for small $\operatorname{Pr}$ and increases asymptotically to $\gamma=90^{\circ}$ for increasing $\operatorname{Pr}$.

At $\gamma<\gamma_{c d}$ only longitudinal rolls occur at onset, since the neutral surface in the $q_{x}-q_{y}-R a$ phase space is not radial symmetric anymore. The neutral curve as a cross section of the neutral surface - depends also on the orientation $\varphi$ and therefore, the critical Rayleigh number and the critical wave number are both functions of the relative orientation $\varphi$ and of the inclination angle $\gamma$.

The onset of rolls with different $\varphi$ and their stability has been investigated in detail for unforced ILC in [47] and [51]. Figure 2.2 shows the critical temperature difference (normalized) and the control parameter $\varepsilon=R a^{I} / 1707.8-1$ (inlet) as a function of the inclination angle $\gamma$ for different orientations $\varphi$. One can clearly see the situation as explained above. For inclination angles smaller than a critical angle $\gamma_{c d}$, longitudinal rolls with their axis parallel to the inclination direction $\left(\varphi=0^{\circ}\right)$ sets in first at $R a_{c}^{I}=1707.8$. For inclination angles $\gamma>\gamma_{c d}$, transverse rolls set in at $R a^{I}<1707.8$. As shown in Fig. 2.2 the marginally stable curves for rolls oriented with angle $\varphi$ is for small $\gamma$ close to $R a_{c}^{I}=1707.8$ and converges for $\gamma \rightarrow 0^{\circ}$ to the isotropic case $(\gamma=0)$. For increasing $\gamma$, the critical Rayleigh number for each orientation angle $R a_{c}^{I}(\varphi)$ increases as well and reaches a maximum of $R a_{c}^{I}(\varphi) \approx 6000$ at an inclination angle $\gamma_{\max }(\varphi)$. A further increase in $\gamma$ reduces again $R a_{c}^{I}(\varphi)$. At $\gamma_{c d} \approx 80^{\circ}$, the green curve for longitudinal rolls crosses the blue curve for transverse rolls. For $\gamma>\gamma_{c d}$, the onset curve for transverse rolls is below all other onset curves. Therefore, in this regime transverse rolls become first unstable. This codimension-two point $\gamma_{c d}$ depends on $\operatorname{Pr}$ and is shifted for higher values of $\operatorname{Pr}$ to higher inclination angles $(\gamma)$ and for lower $\operatorname{Pr}$ to lower $\gamma$ (see Fig. 1.7b).

The critical wave number for a specific inclination angle $\gamma$ depends on $\varphi$ as well, as shown in Fig. 2.3. It is worth noting that the minimum of the critical wave number for each $\varphi$ occurs at angles for which $R a_{c}^{I}(\varphi)$ has its inflection point. 


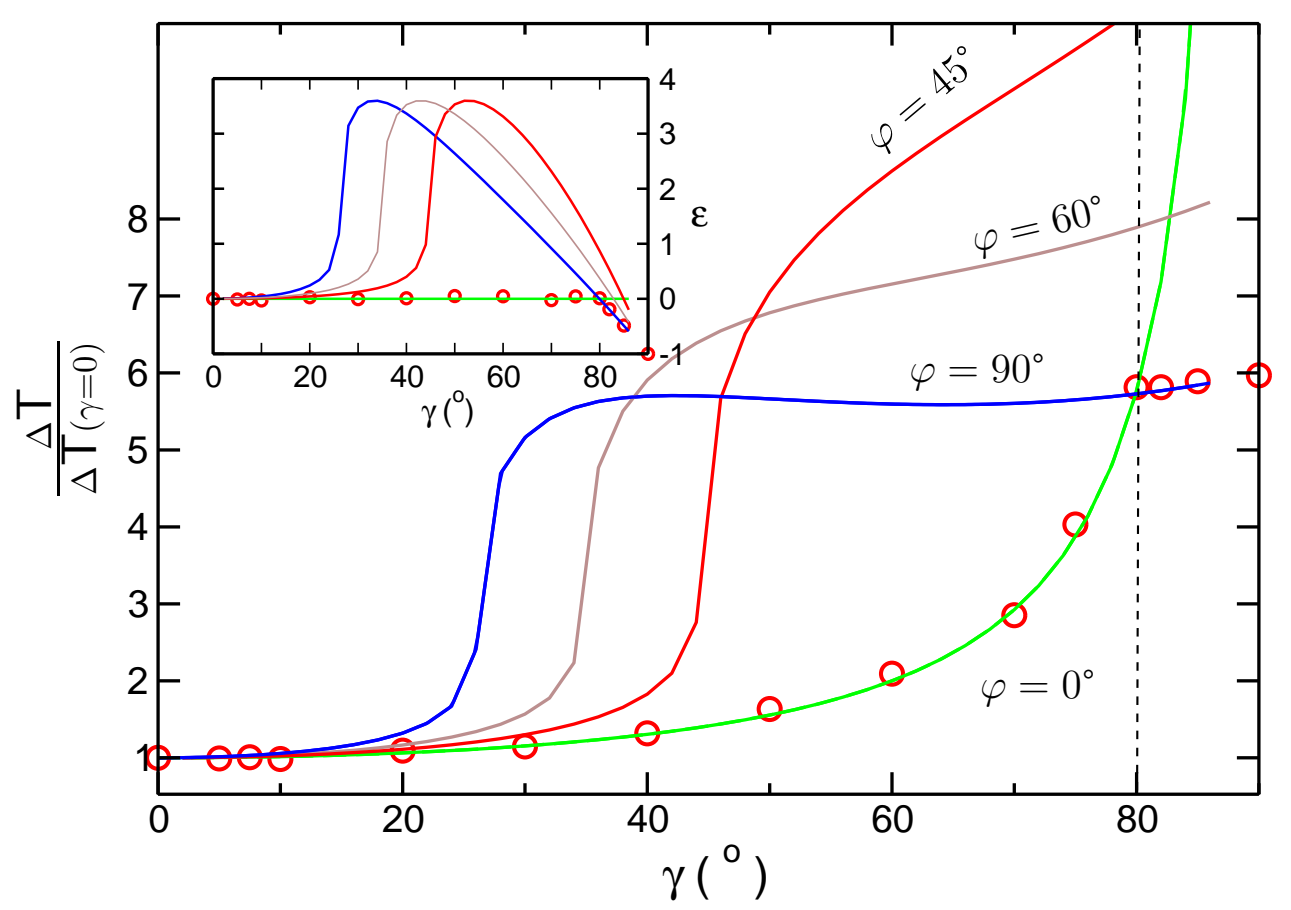

Figure 2.2: Onset of straight rolls as a function of inclination $(\gamma)$ for different angles $\varphi$ and $\operatorname{Pr}=1.3$. Red circles mark experimental values obtained from a reference cell (see later Chapters). At the y-axis, the normalized temperature difference is plotted. The inlet shows the same plots for the control parameter $\varepsilon=R a^{I} / R a_{c}^{I}-1$. The dashed line marks the codimension-two point at $\gamma_{c d} \approx 80^{\circ}$. 


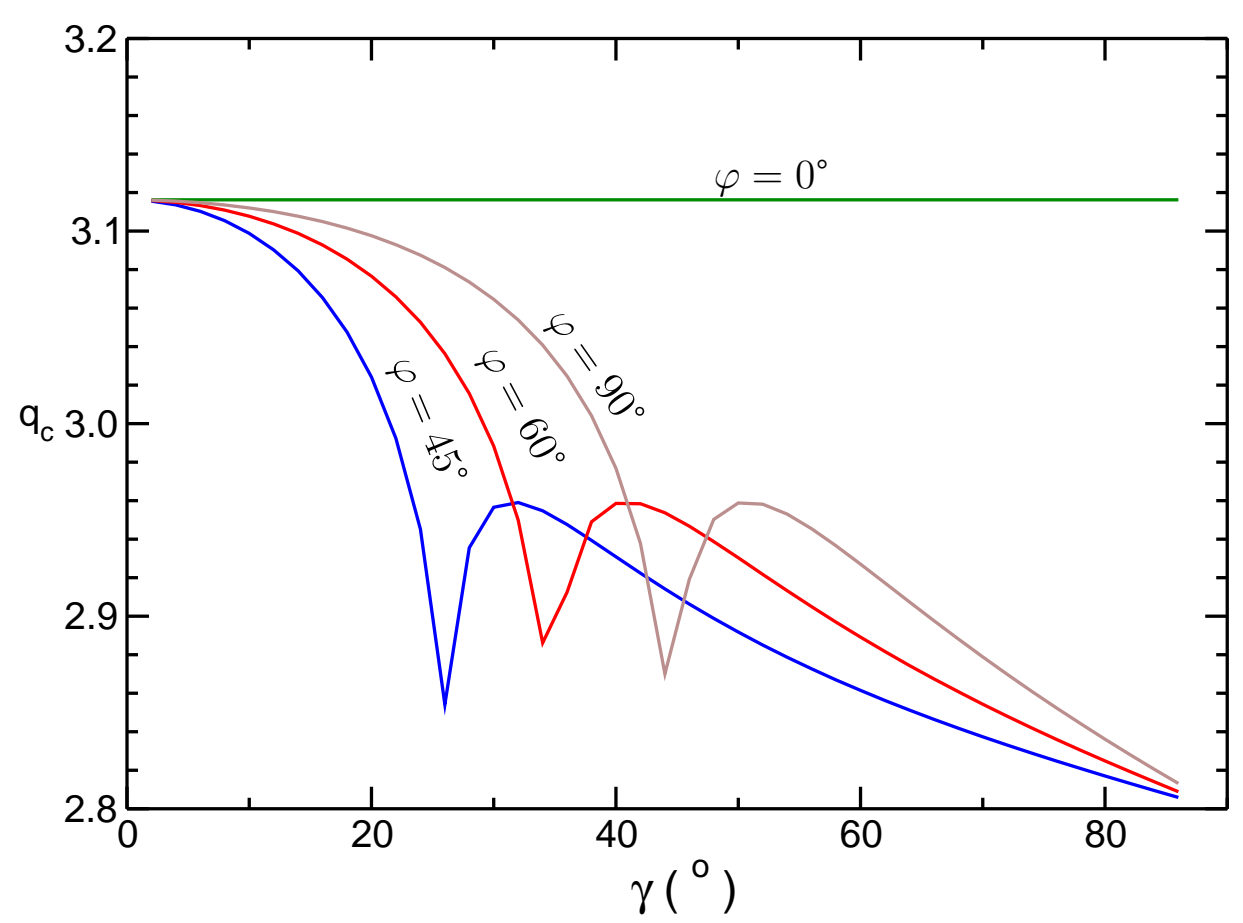

Figure 2.3: Critical wave number as a function of $\gamma$ and $\varphi(\operatorname{Pr}=1.3)$.

Introducing a boundary forcing to ILC can be done in a similar way as for the horizontal case. In addition, a new degree of freedom occurs, which is due to the alignment of the forcing with respect to the gravity in plane component. This degree of freedom is the angle $\varphi$ between the gravity component and the forcing pattern (see Fig. 4.1). Therefore, for the forcing case the modulation part of the temperature has to be written as:

$$
T_{\text {mod }}=-\cos \left(q_{f}(\sin (\varphi) x+\cos (\varphi) y)\right) \frac{\sinh \left(q_{f}(z-d / 2)\right)}{\sinh \left(q_{f} d\right)}
$$

The following procedure is analog to the horizontal forcing case. Every $\theta$ in the differential equations for the unforced inclined layer convection (see Appendix) has to be replaced by $\theta+\delta \frac{R a^{I}}{\Delta T} T_{\text {mod }}$. The resulting equations are highly complex and can be solved numerically. 


\subsection{Numerical Methods}

In general experimental results are presented in this thesis. However, often numerical calculations are used to compare the experimental results with theoretical predictions. The corresponding numerical codes were written by Prof. Pesch and his co-workers at the university of Bayreuth. A detailed description can be found in [51] and [73].

For a vector $\vec{X}=(\theta, f, g)$, the Boussinesq equations can be written in a simplified form as: ${ }^{3}$

$$
\frac{d}{d t} C \vec{X}=L \vec{X}+N(\vec{X} \mid \vec{X})
$$

Here $C$ and $L$ are linear differential operators and $N(\vec{X} \mid \vec{X})$ includes all non-linear terms.

The common procedure to find a stationary solution of Eq. 2.35 is a Galerkinapproach. Thereby the spatial dependencies of $\vec{X}$ are expanded in terms of a normalized orthogonal system. It is important that the chosen basis functions fulfill the boundary conditions. In the $\mathrm{x}$-y-plane one uses Fourier functions as a normalized orthogonal basis, while in z-direction harmonic functions $\left(S_{n}(z)=\right.$ $\sin (n \pi(z-1 / 2)))$ are used for the fields $\theta$, and $g$ and Chandrasekhar functions $C_{n}(z)$ for $f$ (introduced in [52]):

$$
\begin{aligned}
\theta_{0}(x, y, z) & =\sum_{n=1}^{m o z} \sum_{m=-i q}^{i q} \theta_{n m} S_{n}(z) \exp \left[\operatorname{im}\left(q_{x} x+q_{y} y\right)\right] \\
f_{0}(x, y, z) & =\sum_{n=1}^{m o z} \sum_{m=-i q}^{i q} f_{n m} C_{n}(z) \exp \left[\operatorname{im}\left(q_{x} x+q_{y} y\right)\right] \\
g_{0}(x, y, z) & =\sum_{n=1}^{m o z} \sum_{m=-i q}^{i q} g_{n m} S_{n}(z) \exp \left[\mathrm{i} m\left(q_{x} x+q_{y} y\right)\right]
\end{aligned}
$$

moz and $i q$ are cut-off parameters and determine the number of Chandrasekhar functions, harmonic functions and Fourier modes, that are taken into consideration. The ansatz is then included into the stationary equation:

$$
0=L \vec{X}_{0}+N\left(\vec{X}_{0} \mid \vec{X}_{0}\right) .
$$

The nonlinear differential equation becomes a nonlinear algebraic equation and can be solved iteratively with a Newton method (see also [34]).

\footnotetext{
${ }^{3}$ This described approach here is valid for any Boussinesq equations, no matter whether a forced or an unforced, an inclined or a horizontal system is considered.
} 
The stability of the stationary solution $\vec{X}_{0}$ can be checked via a Flouqet ansatz. For this ansatz one assumes a small perturbation of $\vec{X}_{0}$ :

$$
\vec{X}=\vec{X}_{0}+\vec{X}_{s}
$$

and can then linearize Eq. 2.35 around $\vec{X}_{0}$. The ansatz for the perturbation has to use the same system of basis function, since also for the small perturbations, the boundary conditions have to be fulfilled:

$$
\begin{aligned}
& \theta_{s}(x, y, z)=e^{\sigma t} \exp [\mathrm{i}(\vec{s} \vec{r})] \sum_{n=1}^{m o z} \sum_{m=-i q}^{i q} \theta_{n m}^{s} S_{n}(z) \exp [\mathrm{i} m(\vec{q} \vec{r})] \\
& f_{s}(x, y, z)=e^{\sigma t} \exp [\mathrm{i}(\vec{s} \vec{r})] \sum_{n=1}^{m o z} \sum_{m=-i q}^{i q} f_{n m}^{s} C_{n}(z) \exp [\mathrm{i} m(\vec{q} \vec{r})] \\
& g_{s}(x, y, z)=e^{\sigma t} \exp [\mathrm{i}(\vec{s} \vec{r})] \sum_{n=1}^{m o z} \sum_{m=-i q}^{i q} g_{n m}^{s} S_{n}(z) \exp [\mathrm{i} m(\vec{q} \vec{r})]
\end{aligned}
$$

In this way one can calculate the growth rate $\sigma$ for several Floquet vectors $\vec{s}$. If the real part of $\sigma$ is positive for any perturbation with $\vec{s}$, the solution $\vec{X}_{0}$ is unstable for perturbations with $\vec{s}$. 


\section{Chapter 3}

\section{The Experiment}

\subsection{Overview of the Gas Convection Apparatus}

The basic concept of the convection apparatus used for the present work was developed in Guenter Ahlers' lab at the UCSB in Santa Barbara by J. R. de Bruyn and E. Bodenschatz [74]. Later on, Brendan Plapp refined the design and built a new apparatus at Cornell University, under the supervision of E. Bodenschatz [75]. Several students and postdocs used this apparatus for research on Rayleigh-Bénard convection [49, 45].

A sketch of the convection apparatus is shown in Fig. 3.1. Pressurized $\mathrm{CO}_{2}$ gas at 48-50 bar is used as a working (convecting) fluid. The use of gases at high pressure gives rise to several advantages in comparison to using a liquid, like water for example. Due to a large expansion coefficient and a low kinematic viscosity of pressurized gases, $R a$ is always larger at the same $\Delta T$ as in water for example. Therefore, one can work with comfortable temperature differences of the order of a few Kelvin and at the same time uses very thin cells of less then a millimeter. Such small cell heights are an advantage since one wants to have large aspect ratios in order to minimize the influence of the lateral boundaries. In addition, the vertical diffusion time scale $\left(\tau_{\kappa}=d^{2} / \kappa\right)$, is of the order of a seconds and hence the time until the system equilibrates is short enough to investigate nontransient states. If one considers water instead $(\operatorname{Pr}=7)$, one needs a cell height of a centimeter in order to get a similar value for the critical temperature difference $\left(\Delta T_{c} \approx 1.3 \mathrm{~K}\right)$ as in the compressed gas experiments. The longest time scale, the thermal diffusion timescale increases to $\tau_{\kappa} \approx 108 \mathrm{~s}$. In experiments with a similar aspect ratio $L / d$ one end up with times of about $108 \mathrm{~s} \cdot 80^{2}=691200 \mathrm{~s}=192 \mathrm{~h}$, which one has to wait until a pattern reaches its steady state. Transient states are stable for such a long time that it is almost impossible to see in the experiment non-transient states beside simple straight rolls.

Due to the compressibility of gases one can also change their density by increasing 


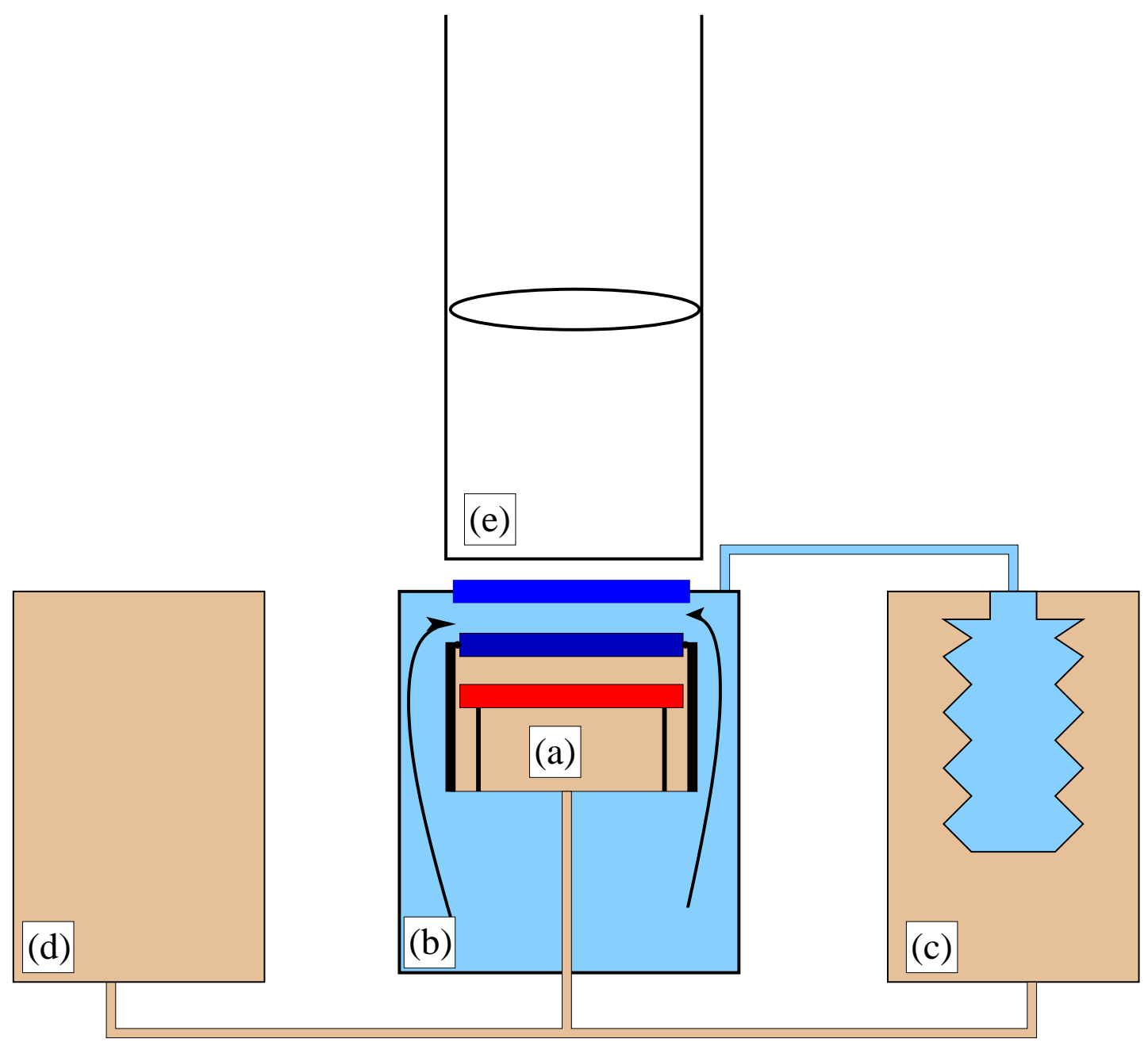

Figure 3.1: Sketch of the convection apparatus with: Convection cell (a) sitting in the main pressure vessel (b), pressure equilibration vessel with bellows (c) and pressure regulation vessel $(\mathrm{d})$ and shadowgraph to image the convection flow pattern (e).

or decreasing the pressure, and thus, change the Prandtl number slightly (within a range of $0.7<\operatorname{Pr}<1.3$. The experiments presented here, were done at room temperature $\left(25^{\circ} \mathrm{C}\right.$ and $\left.27^{\circ} \mathrm{C}\right)$, and $\mathrm{CO}_{2}$ under a pressure of around $48 \mathrm{bar}$ resulting in a Prandtl number of $\operatorname{Pr}=1.3$.

The gas is confined between a single crystalline silicon bottom plate (red in Fig. 3.1) and a single crystalline sapphire top plate (dark blue in Fig. 3.1), each having a diameter and thickness of $101.6 \mathrm{~mm}$ and $9.5 \mathrm{~mm}$, respectively ${ }^{1}$. Both plates have surfaces polished to a flatness of $\lambda / 4(\lambda \approx 633 \mathrm{~nm})$. By using monochromatic laser light the plates were aligned to be parallel to within $0.5 \mu \mathrm{m}$. The bottom plate

\footnotetext{
${ }^{1}$ The polished plates were bought by TYDEX, J.S.Co. St. Petersburg, Russia
} 
is heated by a Kapton-film heater (90 $\Omega$, Vicent Industrietechnik, Lüdenscheid), attached to the lower surface of the bottom plate. The lateral boundaries of the cell are made out of paper slips with a thickness of $80 \mu \mathrm{m}$ each. It was found that by using these slips the thermal properties of the side walls matches best with the thermal properties of the gas and the influence of the boundaries to the flow is minimized [74, 75].

A shadowgraph image of the convection cell is shown in Fig. 3.2. For the boundary forcing experiments presented in the Chapters 4 and 5 the cell was square with side length of $46 \mathrm{~mm}$. Next to it, a smaller square cell was placed with $19 \mathrm{~mm}$ side length, without forcing. This cell served as a reference cell. In previous (unforced) experiments in the same convection system, the cell height was determined via laser interferometry. In these measurements the optically measured cell height always corresponded well with the critical temperature differences so that the critical Rayleigh number was always close to $R a_{c} \approx 1708$. Unfortunately interferometry cannot be used in connection with a modulated bottom plate. Instead, the cell height was calculated over the critical temperature difference at convection onset in the reference cell:

$$
d=\left(1708 \frac{\nu \kappa}{g \alpha \Delta T_{c}}\right)^{1 / 3}
$$

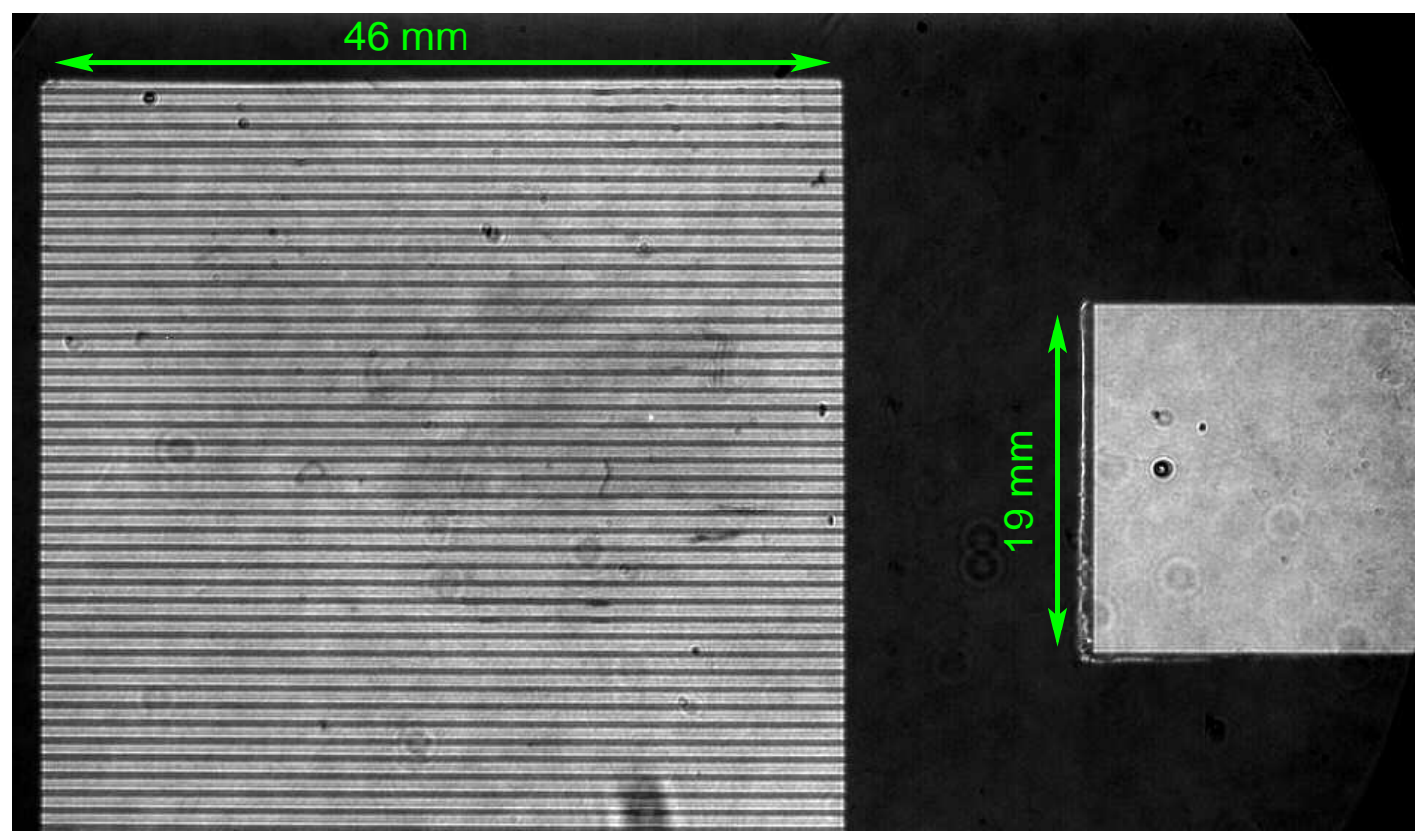

Figure 3.2: Shadowgraph image of the forced cell (left) and the smaller unforced reference cell (right). The dark lines in the forced cell are the SU-8 forcing ridges. 
The convection cell ((a) in Fig. 3.1) sits in a water filled pressure vessel ((b) in Fig. 3.1). The water flows over the top surface of the top plate and cools it, while the other sides of the cell are thermally well insulated against the surrounding cooling water. For precise temperature measurements of the bottom plate, two semiconductor thermistors (NTC-thermistor, $200 \mathrm{k} \Omega$ at $20^{\circ} \mathrm{C}$ ) are attached to its lower surface - one at its center and the other at half way to the edge. Three similar thermistors located at different positions inside the bath measure the temperature of the bath and hence the temperature of the upper surface of the top plate. The heat conductivity of the sapphire and silicon plate are $35 \mathrm{~W} / \mathrm{m} \cdot \mathrm{K}$ and $148 \mathrm{~W} / \mathrm{m} \cdot \mathrm{K}$, respectively [76] and hence more than 1500 times larger than that of $\mathrm{CO}_{2}\left(0.023 \mathrm{~W} / \mathrm{m} \cdot \mathrm{K}\right.$ at $25^{\circ} \mathrm{C}$ and $\left.48 \mathrm{bar}\right)$ [77]. The temperature measurement takes place at the top side of the top plate and the bottom side of the bottom plate, resulting in an overestimation of the temperature difference of $\approx 0.9 \%$ (for $d=$ $540 \mu \mathrm{m}$ and $\Delta T=\Delta T_{c}$ ). In all experiments, this was taken into consideration and the real temperature difference was adjusted by the measured values, assuming a conduction state. For the convective state this recalculation still overestimates the real temperature difference, since the energy flow is larger as in the conduction state. This energy transport rate cannot be calculated easily and hence was not taken into consideration.

A heat exchanger, connected to a NESLAB thermo bath circulator (model RTE7) and an additional heater inside the bath, makes it possible to set the temperature of the cooling water and thus, the temperature of the top plate. In order to distribute the heat and to achieve a homogeneous temperature field inside the cooling bath, an aquarium pump produces a turbulent flow via a flow distributor, to the top plate.

Two other pressure vessels are also part of the system. One vessel is a regulation vessel for the gas pressure inside the apparatus ((d) in Fig. 3.1). It is a reservoir for the working gas with a heater inside and a heat exchanger connected to a NESLAB bath circulator. The temperature inside this vessel is set to the gasliquid transition temperature and thus, liquid $\mathrm{CO}_{2}$ accumulates inside the vessel. A slight change in temperature changes the vapor pressure and thus, the pressure of the working gas can be controlled. After filling the water side of the system completely, the gas side is pressurized with $\mathrm{CO}_{2}$. A bellows in a third vessel ((c) in Fig. 3.1) is necessary to compensate the small decrease of the water volume due to the weak but finite pressurization and thus, avoid pressure differences between the gas and the water side.

A digital multimeter (Keithley 2001) measures the resistance of each thermistor with a resolution of up to 7.5 digits. It is located in a thermally insulated box at a constant temperature in order to avoid temperature induced changes of the resistance of the measurement circuit. The resistance values are sent by the Keithley 
via a GPIB interface to the controlling Linux-PC ${ }^{2}$, and are converted there to temperature values via a calibration table. Using Proportional-Integral-Differential (PID) algorithms, heating power for bath and bottom plate are calculated, and the corresponding voltage is applied at the output of the D/A card of the controlling PC. The output voltage of the computer controls three DC-power supplies, which in turn drive the bath and bottom plate heaters. Pressure regulation is done in the same way. The Keithley 2001 multimeter reads the output voltage of a Sensotec pressure sensor (model S-TJE) and sends these information to the controlling PC where the actual pressure is calculated. Via a PID algorithm the PC calculates a new voltage for the heater inside the pressure regulation vessel.

In this way, the temperature difference of the two plates can be set to a chosen value within a precision of $\pm 0.5 \mathrm{mK}$, and the pressure is held constant to within 0.01 bar. Note that the accuracy mentioned here only refers to the temperature difference and not to the absolute temperature. A calibrated PT-100 (PT-103-70H, LakeShore) was used to calibrate the thermistor against an absolute temperature with an error of less than $25 \mathrm{mK}$.

The main pressure vessel together with the convection cell is mounted on the foot of an old milling machine which makes rotation of the vessel about two axes possible. With a weight of approximately $1000 \mathrm{~kg}$, this foot gives enough stability to incline the whole experiment to angles $\gamma$ up to $100^{\circ}$. An image of the whole apparatus is shown in Fig. 3.3.

Visualization of was done with a shadowgraph ((e) in Fig. 3.1) and will be explained in Sec. 3.3.1.

\subsection{Surface Corrugation}

Forcing the convection pattern is achieved by modifying the bottom plate surface. Via photo lithography a periodic surface corrugation was printed on the bottom plate as shown in Fig. 3.4. The one-dimensional corrugation consists of a long rectangular stripe every millimeter with a height of $65 \mu \mathrm{m}$ and a width of $100 \mu \mathrm{m}$. The epoxy based transparent photoresist $S U-8$ by MicroChem Corp. ${ }^{\mathrm{TM}}$ was used for this purpose. For this commonly used negative photoresist, well developed recipes are provided by MicroChem Corp. ${ }^{\top M}$ and can be found on their website [78]. Initially all these recipes were developed for lithography on silicon substrates which are less than a millimeter thick, and therefore, these recipes had to be adjusted to work on our $10 \mathrm{~mm}$ thick bottom plate. These adjustments mainly affect the backing and cooling times due to the much higher heat capacity of our

\footnotetext{
${ }^{2}$ In former experiments the real time operating system QNX was used. However, in experiments presented here no time critical phenomena were investigated and the precision given by LINUX was sufficient for our purposes.
} 


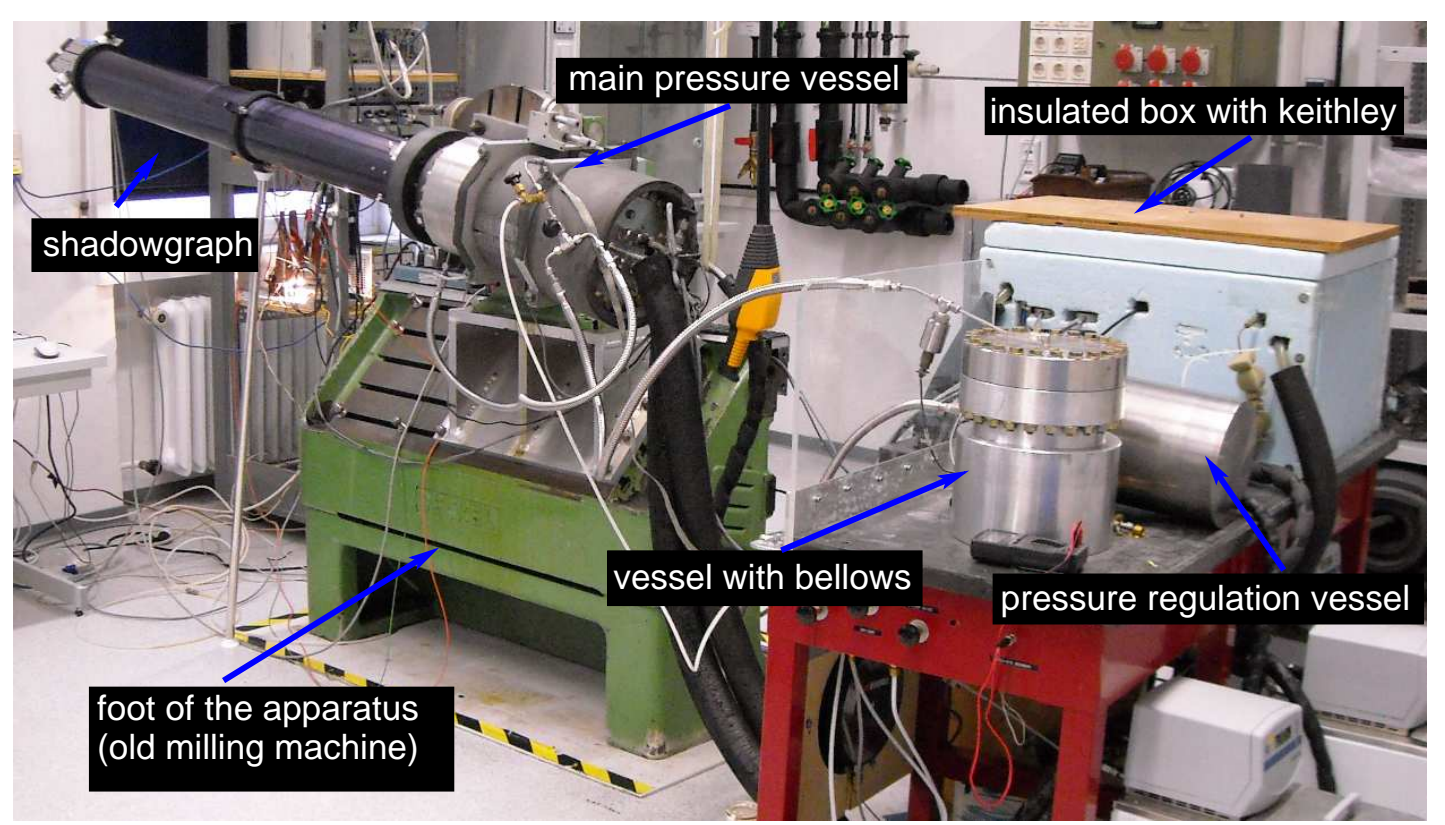

Figure 3.3: Overview of the experimental apparatus

thick plate. Adjusted recipes were developed by John McCoy and can be read in detail in his thesis [68]. Nevertheless, we give a short overview of the lithographic process here, since some additional changes to his recipe were made because some special problems occurred.

The lithographic process consists of three main processes: Spin coating, exposure and developing. In addition baking is done in order to evaporate solvent of the photoresist and to accelerate cross linking of the exposed molecules.

Spin coating. The purpose of spin coating is the deposition of a thin homogeneous layer of photoresist on the substrate. Therefore, the substrate has to be clean and free of particles or residues like fat or oil which might alter the wetability. Thus, the substrate has to be cleaned first with acetone and isopropanol. A good way to do this is by using the spin coater at a rotation speed of about $2500 \mathrm{rpm}$. After the coater has reached its maximum speed, one squirts a steady stream of acetone to the center of the substrate for approximately $10 \mathrm{~s}$. While squirting acetone on the rotating substrate, one begins to add a steady stream of isopropanol and to hold both for a few seconds. The acetone stream can be stopped and isopropanol alone is squirted for another $10 \mathrm{~s}$. This procedure should remove most of the organic residuals and produce a sufficiently clean substrate surface. Due to this cleaning process the silicon surface often becomes hydrophilic because of water molecules attached to it. In order to remove this thin water layer, the wafer has to be heated up to $200^{\circ} \mathrm{C}$ for about $10 \mathrm{~min}$. It is very important to wait after the heating is finished, until the plate cooled down 


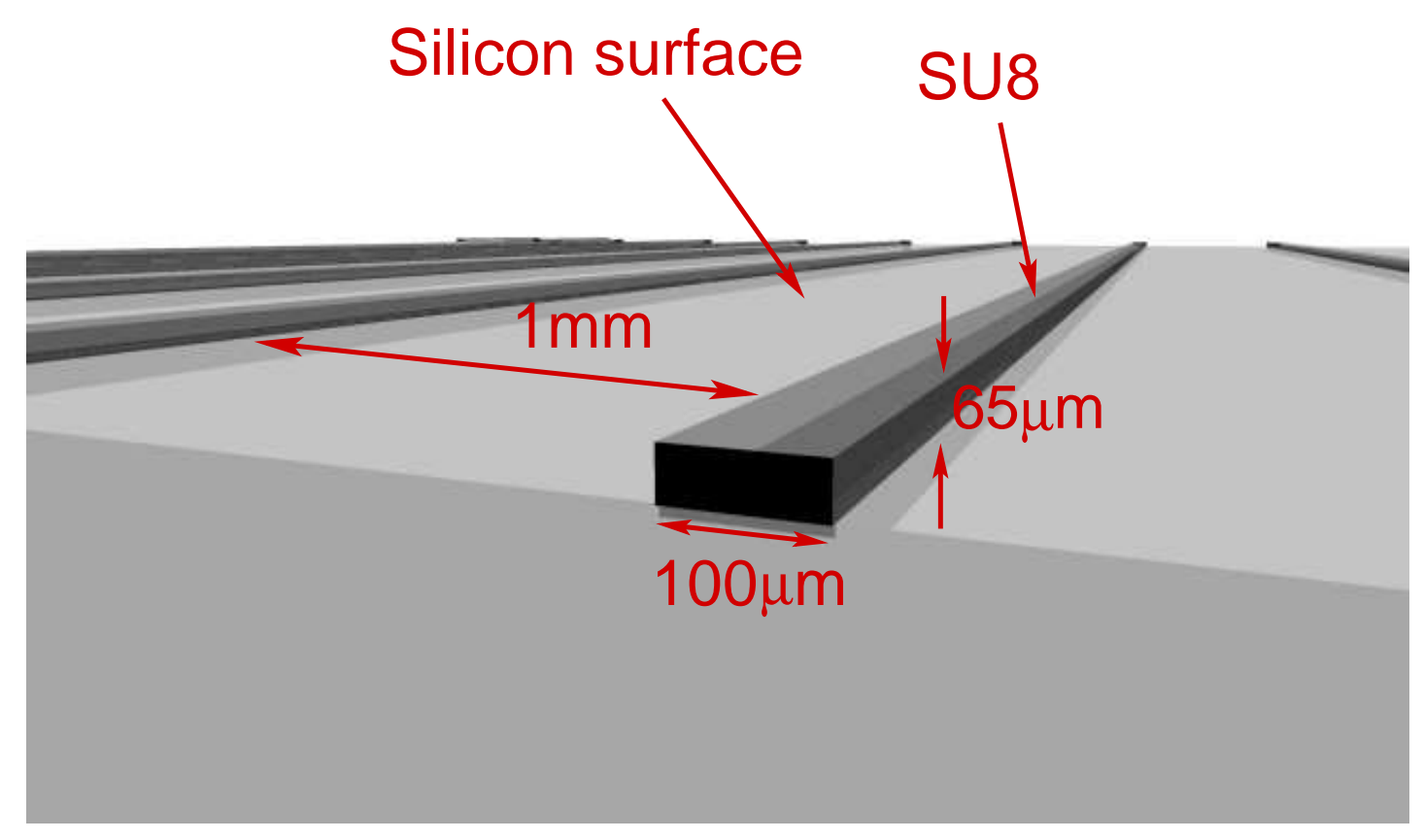

Figure 3.4: Schematic of the surface of the bottom plate.

to room temperature $\left(\approx 22^{\circ}\right)$, before starting with spin coating of the SU-8 onto the wafer. Since the viscosity of SU-8 depends on the temperature, the film thickness will be too low if the plate is still warm. It turned out that the baking in order to remove the adhesive water is an essential step. Without it, the SU-8 will not wet the substrate properly or might start to dewet the substrate during the subsequent baking process.

The main component of a spin coater ("Ramgrabber" - SIEMENS ) is the chuck - a rotating table with a hole in its center. A vacuum pump connected to the hole creates a low-pressure and thus sucks the wafer onto the chuck. Side-pins hold the wafer in lateral direction and prevent it from flying away at high rotation speed. Unfortunately, the diameter of our silicon bottom plate is slightly larger than the standard silicon wafer and so does not fit onto the chuck. A suitable adapter was designed and built out of aluminum.

If the silicon plate is clean and dehydrated, spinning of the SU-8 can be performed. The high viscosity of the SU-8 makes it difficult to pour the fluid onto the substrate. Care has to be taken so that no bubbles form during this process, 
since they lead to strong inhomogeneities in the film thickness. The best way is to pour the SU-8 directly out of the bottle onto the center of the substrate. After poring a sufficient amount of SU-8 onto the wafer, the spin coating should be performed in two steps. The first step, in which the rotating speed is very low, distributes the SU-8 over the whole substrate. In the second step, the speed is set to the required value to decrease the film thickness to the desired level. If everything was done carefully, the result of this procedure will be a bottom plate covered with a homogeneous film of viscous SU-8. Due to light interferences on the SU-8 surface one can see with the naked eye how homogeneous the film thickness is and where inhomogeneities are located.

Soft bake. Now the bottom plate has to be backed to evaporate the solvent and to densify the film. This step is performed on hot plates whereas care has to be taken that the plates are leveled precisely. Since a very fast rise of temperature results in tensions and cracks within the SU-8 layer, heating has to be done either in several steps, or even better, by slowly ramping the temperature until the desired temperature is reached. Since the bottom plate has a high heat capacity and cannot be heated up as quickly as a $0.5 \mathrm{~mm}$ thick silicon wafer, heating in two steps turned out to be fine. In the first step, the Si-plate is baked for 8:20 min at $70^{\circ} \mathrm{C}$. In the second step, the temperature is set to $102^{\circ} \mathrm{C}$ and the Si-plate is baked for $23 \mathrm{~min}$, which is sufficient to evaporate most of the solvent. Instead of rising the temperature of the plate for the second step, two hot plates were used and the Si-plate was moved after 8:20 min from the colder to the hotter plate.

Exposure. When the silicon plate has reached room temperature $\left(\approx 22^{\circ}\right)$ after removing it from the hot plate, exposure can be performed. SU-8 is a negative photoresist, meaning that SU-8 which was exposed to light will cross-link during the following baking, while the non-exposed areas can be dissolved again and washed away by a corresponding developer. SU-8 strongly absorbs UV-light with a wavelength smaller than $400 \mathrm{~nm}$. A mercury lamp is used for illumination of the SU-8, which has a sharp line at $\lambda=365 \mathrm{~nm}$ (i-line).

A mask, which is made out of glass covered with a thin chromium layer, was designed and made by J. McCoy at the Cornell Nanofabrication Facility (CNF). Although the mask aligner $\left(\mathrm{EV} 620-\mathrm{EVG}^{\mathrm{TM}}\right)$, was designed to work with wafers up to $5 \mathrm{~mm}$, no problems occurred while using it with the $10 \mathrm{~mm}$ thick bottom plate. Using the mask aligner is a straightforward thing to do as long as all the non-specific parameters ${ }^{3}$, are set up correctly. The only thing the user has to do is to set the correct exposure time. Too short an exposure will only cross-link the upper layer of the SU-8 but not the lower one so that the SU-8 will not adhere to

\footnotetext{
${ }^{3}$ Non-specific parameter are all parameter which should not be changed at all, like the applied nitrogen pressure, the light intensity or the vacuum pressure. Specific parameter on the other hand have to be set for each exposure to a convenient value. Such parameters are e.g. the exposure time, soft- or hard-contact mode and so on. All important values for the non-specific parameter can be found in the manual [79].
} 
the substrate and will be washed away during the developing process. Too long an exposure, on the other hand, will decrease the sharpness of vertical structures of the photoresist and will also enlarge the exposed areas in lateral direction. MicroChem recommends for a SU-8 film thickness of $65 \mu \mathrm{m}$ an exposure dose of $250-450 \mathrm{~mJ} / \mathrm{cm}^{2}$. It turned out, that doses below $450 \mathrm{~mJ} / \mathrm{cm}^{2}$ where to low and the exposed SU-8 was always removed during the development process. Therefore a dose of $450 \mathrm{~mJ} / \mathrm{cm}^{2}$ was used. With the lamp (mercury lamp with ) set to $350 \mathrm{~W}$ and a right adjustment of the mask aligner the dose was reached by exposure of the light with $10 \mathrm{~mW} / \mathrm{cm}^{2}$ for $45 \mathrm{~s}$.

Developing. After exposure, another backing has do be done in order to selectively cross-link the exposed portions of the film. Again, heating the hot plates in at least two steps is important to avoid cracks in the film. Backing is followed by the final development process. The specially designed SU-8 developer by MicroChem Corp. ${ }^{T M}$ was used to remove the parts of the film which have not been cross-linked. While the bottom plate bathes in the developer for a couple of minutes, agitating the bath container helps to dissolve the SU-8 and to remove dissolved material from the substrate. The plate can now be taken out of the developer bath and must be rinsed by a stream of isopropanol to wash away developer with the dissolved SU-8. When the developer is washed away the structured substrate should be dried immediately by dry nitrogen. If a white film occurs on the substrate during rinsing, development was not fully accomplished. This is in general not a problem. One can repeat the whole process in order to remove the residual SU-8.

OmniCoat. Thin silicon wafers usually used in photo lithography cost around 10-30 Euro. If the lithographical process was not successful, one can start again with a new wafer and discard the old one. On the other hand, $10 \mathrm{~mm}$ thick high quality single crystalline wafers as used for this experiment are significantly more expensive (380 Euro, by TYDEX, J.S.Co, St. Petersburg, Russia) and the delivery time of these plates is on the order of several months. Therefore, it is important to remove the SU-8 from the substrate without destroying its surface. For that purpose a very thin layer $(<1 \mu \mathrm{m})$ of OmniCoat (by MicroChem Corp. ${ }^{\mathrm{TM}}$ ) was spin cast on the substrate before coating with SU-8. Later on this layer could be removed by using a warm bath of Remover PG (MicroChem Corp. ${ }^{\mathrm{TM}}{ }^{\text {). However, }}$ the surface could not be perfectly restored and was never the same as it was before the first lithographic process. Although with the naked eye no layer was visible anymore, the wetability of the substrate at the areas where the SU-8 was located was reduced and it became difficult to create another homogeneous OmniCoat layer. Another disadvantage of OmniCoat is its solubility. While SU-8 attaches to the silicon surface very well, the OmniCoat was attacked by the $\mathrm{CO}_{2}$ inside our cell and thus the SU-8 stripes started to detach from the substrate after several weeks and a new texturing had to be made.

The surface of the bottom plate surface was later be checked with a white light 
interferometer (Wyko NT1100 by Veeco, Metrology Group) and the exact step height of the surface texture was measured to be $(65 \pm 2) \mu \mathrm{m}$.

Recipe. The following procedure was done in order to produce a texture with a height of $65 \mu \mathrm{m}$ :

1. Clean the substrate

(a) cleaning substrate with acetone and a tissue

(b) spinning with $2500 \mathrm{rpm}$

(c) cleaning with acetone and isopropanol while spinning as described above

(d) baking on hot plate at $\approx 200{ }^{\circ} \mathrm{C}$ for $10 \mathrm{~min}$

(e) let the wafer cool down to room temperature $\left(\approx 22^{\circ} \mathrm{C}\right)$

2. OmniCoat

(a) dispensing OmniCoat on the wafer until at least $50 \%$ of the surface is covered

(b) accelerate with $100 \mathrm{rpm} / \mathrm{s}$ to $500 \mathrm{rpm}$ and hold that speed for $10 \mathrm{~s}$

(c) accelerate with $300 \mathrm{rpm} / \mathrm{s}$ to $3000 \mathrm{rpm} / \mathrm{s}$ and hold for $35 \mathrm{~s}$

(d) put the wafer on the hot plate preheated to $216^{\circ} \mathrm{C}$ and keep it there for $6.5 \mathrm{~min}$

(e) let the wafer cool down to room temperature of $\approx 22^{\circ} \mathrm{C}$

3. Spinning

(a) pore carefully SU-8 onto the wafer (no bubbles !!)

(b) ramp to $500 \mathrm{rpm}$ with $100 \mathrm{rpm} / \mathrm{s}$ and keep the speed for $10 \mathrm{~s}$

(c) increase speed to $1700 \mathrm{rpm}$ with $300 \mathrm{rpm} / \mathrm{s}$ and hold that speed for $35 \mathrm{~s}$

4. Soft Bake

(a) baking for $8: 20 \mathrm{~min}$ at $70^{\circ} \mathrm{C}$

(b) baking for $23 \mathrm{~min}$ at $102^{\circ} \mathrm{C}$

(c) let the wafer cool down $\left(\approx 22^{\circ} \mathrm{C}\right)$

5. Exposure

(a) exposing SU-8 to $450 \mathrm{~mJ} / \mathrm{cm}^{2}$ of UV light with wavelength $365 \mathrm{~nm}$ $\left(10 \mathrm{~mW} / \mathrm{cm}^{2}\right.$ for $\left.45 \mathrm{~s}\right)$ 
6. Post Bake

(a) bake at $70^{\circ} \mathrm{C}$ for $3: 20 \mathrm{~min}$

(b) bake at $102^{\circ} \mathrm{C}$ for $8: 30 \mathrm{~min}$

7. Development

(a) bath the plate in SU-8 developer for $10 \mathrm{~min}$

(b) rinsing with isopropanol

(c) drying with nitrogen

\subsection{Visualization}

\subsubsection{Shadowgraphy}

For flow visualization in transparent media, and in particular for thermal convection close to its onset, the shadowgraph technique is the method of choice $[80,81,74]$. With this technique, the changes of the refractive index in lateral direction are imaged. These changes occur since in a gas the refractive index is a function of its density and therefore depends on temperature field. The areas of cold sinking and warm rising gas can be identified with this technique.

Experiments were done with the shadowgraph arrangement shown in Fig. 3.5. The light of a $100 \mathrm{~W}$ halogen bulb was filtered to become monochromatic $(\lambda=$ $632 \pm 10 \mathrm{~nm}$ ) and was coupled via an optical fiber into the shadowgraph tube. In the shadowgraph tube (see Fig. 3.3), the light is reflected by a pellicle beam splitter onto a large telescope lens with a diameter of $12 \mathrm{~cm}$. The distance of the light path from the exit of the optical fiber to the telescope lens corresponds with its focal length of $600 \mathrm{~mm}$, in order to produce collimated light behind the lens. After passing the top sapphire window of the main pressure vessel and the top plate of the convection cell, the light is refracted due to the spatially periodic modulation of the refractive index of the convective gas. Then it is reflected back by the mirror surface of the bottom plate and passes again through the convecting gas, where it is refracted a second time. The refraction of the light is very weak $\left(d n / d T=3 \cdot 10^{-4}\right.$ for $\mathrm{CO}_{2}$ at 48 bar, $25^{\circ} \mathrm{C}$ [82]), while the distance $z_{0}$ between the cell and the $600 \mathrm{~mm}$ lens is very small, so that the reflected light is still nearly collimated when it passes the telescope lens a second time. Passing through the big lens a second time, the light is now focused towards a digital CCD-camera (Qimaging Retiga 1350EX or Qimaging QICam B) and imaged to the CCD-Chip by a $50 \mathrm{~mm}$ camera lens $\left(\mathrm{NIKON}^{\mathrm{TM}}\right)$.

The top sapphire pressure window is slightly tilted with respect to the optical axis. Therefore, light which is reflected at the top surface of the pressure window 
does not follow the optical axis, but is reflected with an angle to it (blue line in Fig. 3.5). A small pinhole at a distance from the telescope lens of exactly its focal length $(\mathrm{f}=600 \mathrm{~nm})$ makes sure, that the light that is reflected from the top sapphire is blocked out and thus does not disturb the image at the CCD chip.

Below, it will be explained how a laterally periodic temperature field leads to a periodic modulation of collimated monochromatic light. It will also be shown, that the modulation amplitude of the light intensity depends strongly on $z$, which is the distance between the location, where the refractive index is modulated (the convection cell) and the imaging plane. The modulation amplitude is close to zero for $z \rightarrow 0$, then the imaging plane is very close to the convection cell. Since the change in the refractive index with temperature is very low $\left(d n / d T=3 \cdot 10^{-4}\right.$ for $\mathrm{CO}_{2}$ at $48 \mathrm{bar}, 25^{\circ} \mathrm{C}$ [82]), the distance $z$ needs to be large in order to have a significant light intensity modulation. The telescope lens now is important for a second reason. Beside the fact, that it collimates the light from the light source, it also reduces the distance one needs in order to have a significant intensity modulation of the reflected light.

Let's assume that the imaging lens is set up so, that a plane with distance $\Delta z$ below the pin hole is imaged to the CCD-chip. The light modulation at that plane is equivalent to the light modulation at a distance $z$ from the cell if there would be no telescope lens. By applying the equation for thin lenses one can calculate $z$ to be:

$$
z=z_{0}+\frac{f(f-\Delta z)}{\Delta z} \cdot[74]
$$

The distance between the telescope lens and the convection cell is denoted as $z_{0}$ whereas the cell height $d$ is negligible $\left(d \ll z_{0}\right)$. The focal length of the telescope lens is given by $f$, which was $f=600 \mathrm{~mm}$ in the experiment. In other words, the light intensity at the object plane at distance $z$ above the cell is imaged by the telescope lens to the imaging plane at distance $\Delta z$ below the pinhole.

This conversion is important, since one is interested in a quantity that is independent of the optical elements. In theoretical models the light intensity is calculated in dependence of $z$. By changing the position of the imaging plane $\Delta z$ one does change the actual parameter $z$. Changing $\Delta z$ is easily achieved by changing the position of the camera and the camera lens (see Fig. 3.5), which could be done on turning knobs at the outside of the shadowgraph. In the following, when the shadowgraphy is explained more detailed, only $z$ is important and it will be assumed, to consider a system without any optical elements.

The camera is connected via IEEE 1394 FireWire $^{T M}$ to a Macintosh computer where the images are stored in TIFF format and processed later on. The camera is triggered by the controlling LINUX-PC. This PC is only used for controlling temperature and pressure of the experiment, and for triggering the camera. Even though LINUX is not a realtime operating system, it is accurate enough to trigger 


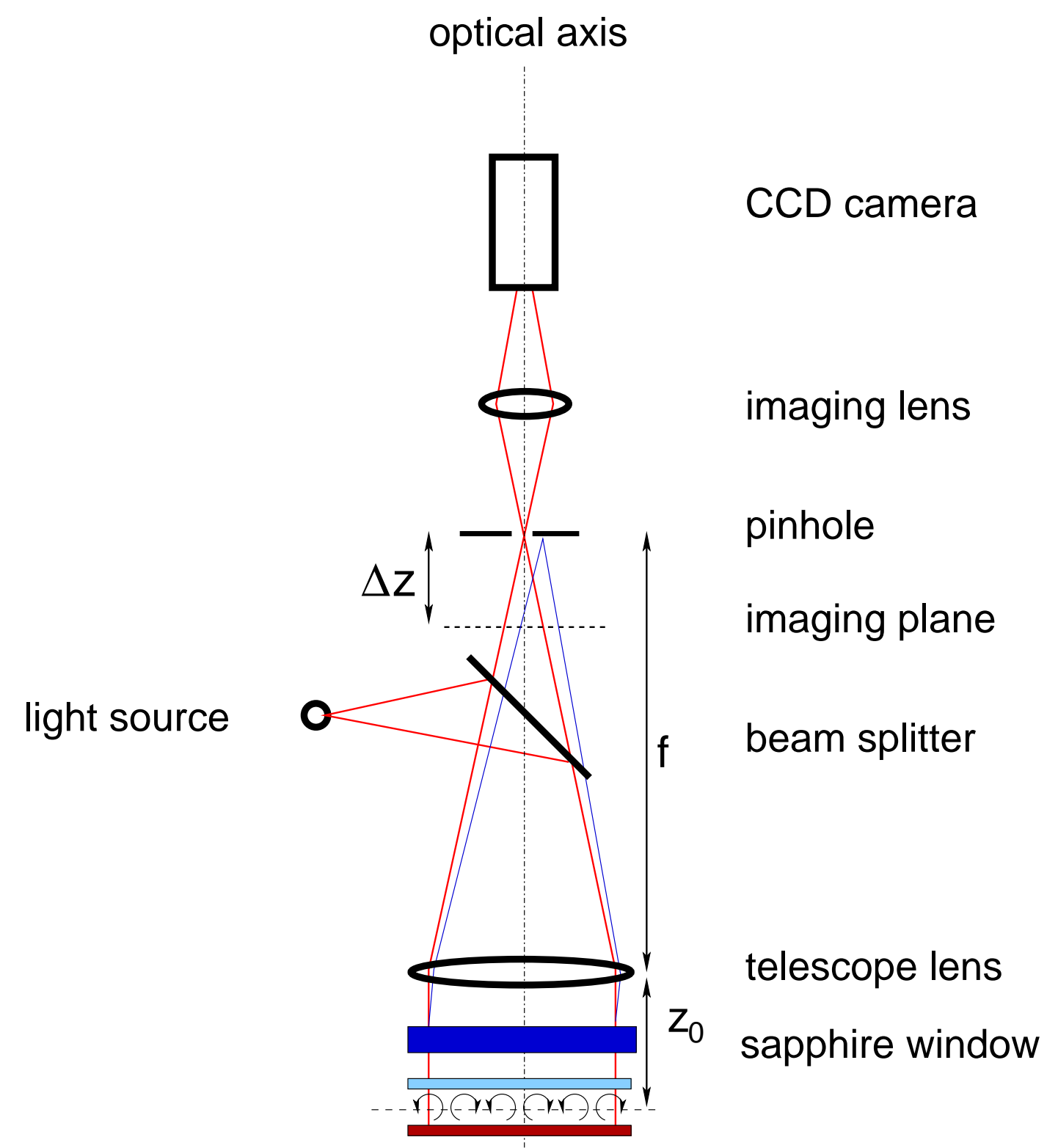

Figure 3.5: Schematic drawing of the shadowgraph arrangement. All optical components are build in a long opaque metal tube. The red lines are representative light rays that are modulated by the convecting gas and finally build up the image in the camera. The blue lines mark the light rays, that are reflected on the top sapphire window. 
the camera up to $10 \mathrm{fps}$. As was checked with an oscilloscope no observable time delay $(<1 \mathrm{~ms}$ ) occurred during the time for a typical experiment.

Shadowgraphy can be understood qualitatively by using geometrical optics [81]. The spatial periodic temperature field of the convection cell results in a periodic density field and thus, in a periodic modulation of the refractive index. Cold areas have a higher density and thus a higher refractive index as warm areas. A change in the refractive index always leads to a refraction of light. From this viewpoint, the periodically modulated field of the refractive index can be seen as an array of small lenses [83]. Collimated light, which passes through the gas, is modulated by that lens array, so that light rays are refracted towards regions of higher refractive index - regions of cold sinking fluid, as shown in Fig. 3.6a. Thus, for a sufficiently small distance $z$ above the cell - how small will be explained later in that section - the intensity of the reflected light is modified in $\mathrm{x}$ - and $\mathrm{y}$-direction so that higher light intensity occurs above cold areas, while low intensity occurs over warm upflow areas. The amplitude of the modulation of the light intensity does depend on $z$. Ray optics predicts a first maximum of the light modulation at a distance $z=z_{\max }^{g}$ which would be the focal length of the lens array (Fig. 3.6a). In fact, at this position a caustic occurs above cold regions, where the calculated light intensity becomes infinite (see e.g. [81]).

These caustics with a infinite intensity are somehow not physical, since at least diffraction effects would limit the energy density. Therefore, a more quantitative view must consider physical optics. This was done for a sinusoidal refraction grating $[84,83]$ and also for thermal convection with higher amplitude, where the modulation of the refractive index is not sinusoidal anymore, but still periodic [83].

In these cases, the occurring light intensity modulation does not only depend on the position of the imaging plane $(z)$, but also on the wavelength of the light. For a one dimensional sinusoidal grating the dimensionless parameter $\rho=z_{\text {max }}^{g} l / \lambda^{2}$ was introduced in [84]. Here, $z_{\text {max }}^{g}$ is the distance from the grating at which caustics would occur regarding geometrical optics, $\lambda$ is the wavelength of the refraction grating (the wavelength of the convection rolls) and $l$ is the wavelength of the light. For large $\rho$ diffraction effects become more important, while for $\rho \rightarrow 0$, the intensity field becomes more similar to that predicted by geometrical optics. Fig. 3.6b was calculated regarding [84] with $\rho=1 / 15$, which is a typical value at convection onset for the experiments shown in this work. ${ }^{4}$ For larger convection strength, this value decreases. As can be seen, for $z>z_{\text {max }}^{g}$ the intensity along a plane does not necessarily correspond anymore with the temperature modulation in the convection cell. Areas above cold regions, that where bright before might become dark and vice versa. One can therefore not relate a bright spot in the

\footnotetext{
${ }^{4}$ The value is calculated for $\mathrm{CO}_{2}$ at $48 \mathrm{bar}, 25^{\circ} \mathrm{C}$ a cell height of $540 \mu \mathrm{m}$ and a light with wavelength $l=630 \mathrm{~nm}$.
} 

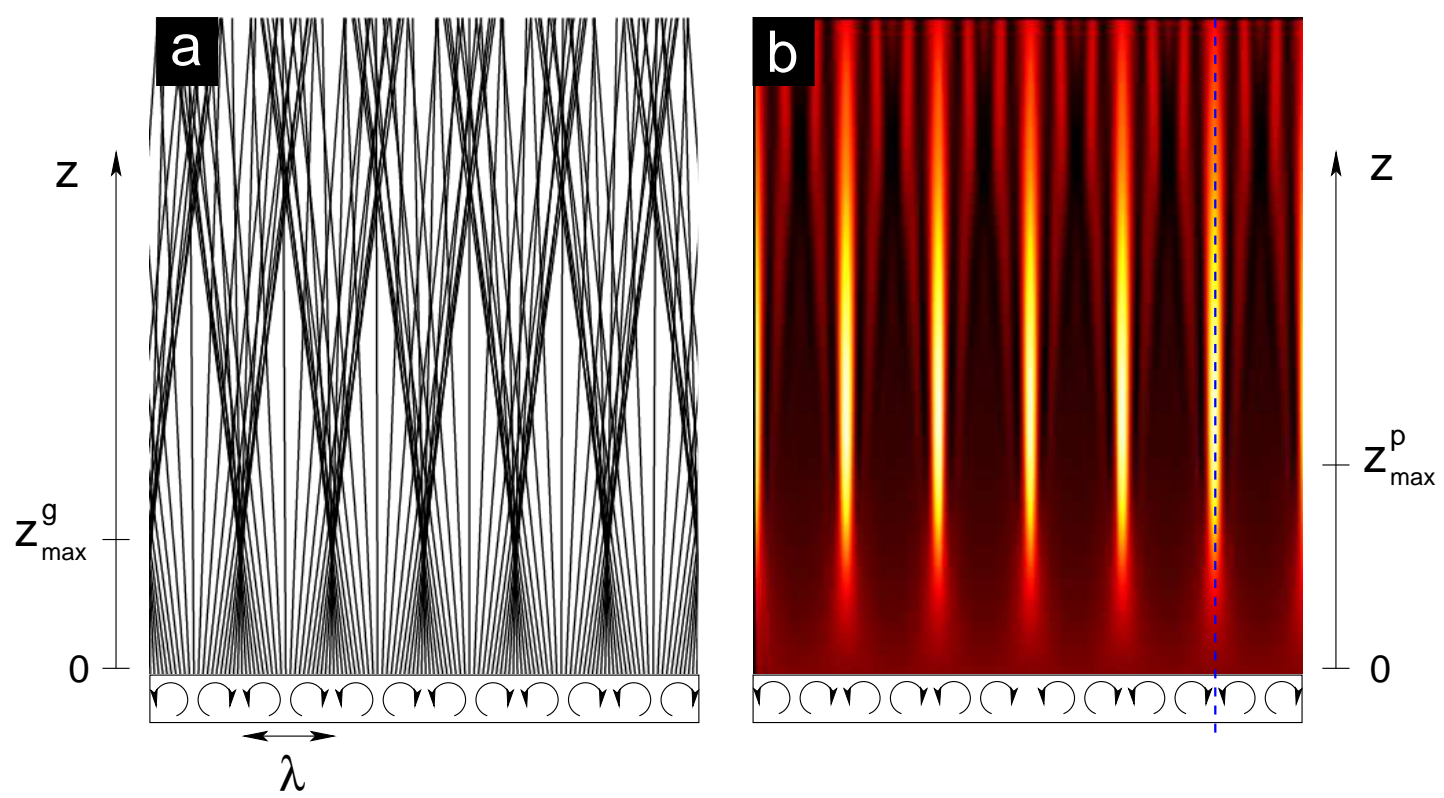

Figure 3.6: Light rays and light intensity due to a sinusoidal modulation of the refractive index in the convection cell. (a) Light rays with caustics at $z_{\max }^{g}$ in the geometrical optics. (b) Light intensity distribution for a physical optics approach. The intensity field was calculated for $\rho=1 / 15$ (see text) regarding [84]. The dashed blue line lies above a cold region. The light intensity along this line is plotted in Fig. 3.7.

shadowgraph image with a cold spot in the convection cell, if one images planes with $z>z_{\text {max }}^{g}$.

In Fig. 3.7, the light intensity above a cold region (along the dashed blue line in Fig. 3.6b) is plotted as a function of $z$, for $\rho=0$ (ray optics), $\rho=1 / 15$, and $\rho=1 / 50$. The light intensity is normalized with the intensity at $z=0$. In all three cases, the intensity grows first with increasing $z$ until it reaches a maximum at $z_{\max }^{g}\left(\right.$ for $\rho=0$ ) and $z_{\max }^{p}$ (for $\rho>0$ ) and then falls down again to reach a local minima. For $\rho>0$ the intensity grows again and reaches a second maximum, smaller than the first one (not shown in Fig. 3.7). Note, that the exact location of the first maximum $\left(z_{\max }^{p}\right)$ increases with increasing $\rho$. Since $\rho$ decreases with increasing convection strength, the location of $z_{\max }^{p}$ also decreases with increasing convection amplitude and moves closer to $z_{\text {max }}^{g}$. As can be seen in Fig. 3.7, geometrical optics approximates the physics well if one images planes that are close enough to the convection cell $\left(z \lesssim 0.7 z_{\text {max }}^{g}\right)$. In all experiments care was taken not to get close to $z_{\max }^{g}$.

Assuming a temperature field $T\left(\vec{x}_{h}, z\right)$, which is periodic in the horizontal direction with wave number $q$, then the shadowgraph intensity $\left(I\left(\vec{x}_{h}, \Delta T\right)\right)$ at a given 


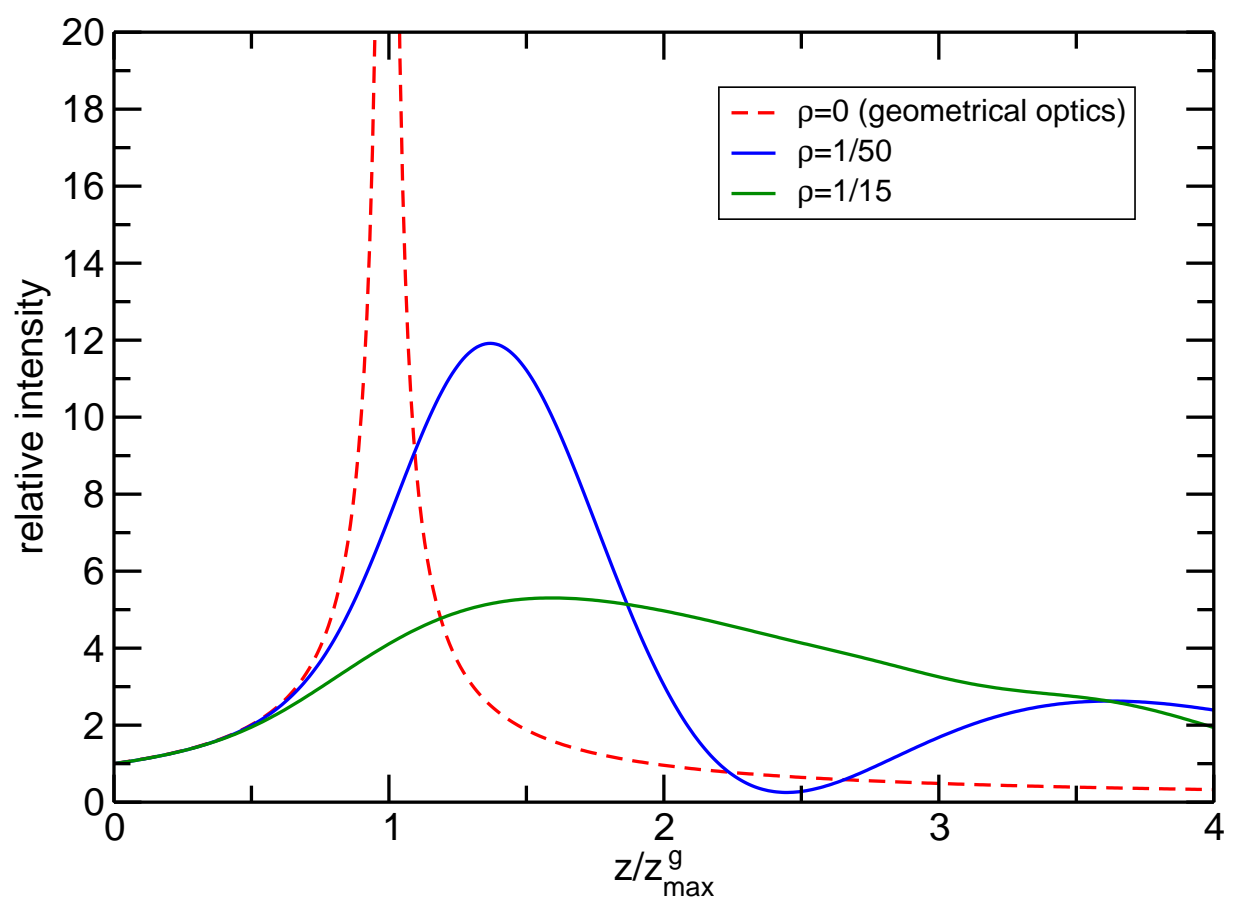

Figure 3.7: Shadowgraph intensity above a cold region (along the dashed blue line in Fig. 3.6) in dependence on the normalized axial distance to the convection cell (from [83]).

distance to the convection cell $(z)$ is given by:

$$
I\left(\vec{x}_{h}, \Delta T\right)=I_{0}\left(\vec{x}_{h}\right)\left(1+S\left(\vec{x}_{h}, \Delta T\right)\right) .
$$

Here $I_{0}\left(\vec{x}_{h}\right)$ is the intensity at $\Delta T=0 \mathrm{~K}$ and $\vec{x}_{h}$ denotes the horizontal coordinates $(x, y)$. If the shadowgraph focuses sufficiently close to the convection cell (small $z)$, the relative modulation of the intensity in real space $S(\vec{x}, \Delta T)$ is for small $\Delta T$ (Boussinesq approximation), proportional to $\langle\hat{T}(\vec{x}, z)\rangle_{d}$, the over the z-direction averaged lateral temperature modulation (averaged over the whole cell height):

$$
S(\vec{x})=G\left(\frac{q}{q_{c}}\right)^{2}\langle\hat{T}(\vec{x}, z)\rangle_{d} .
$$

The lateral temperature modulation is nothing else than the temperature field without the linear conduction profile: $\hat{T}=T-T_{\text {cond }}$. $G$ is a conversion factor, consisting all properties of the optical path including the imaging distance. The factor $q / q_{c}$ comes from the second derivative of the temperature field. 


\subsubsection{Image Processing}

Each image taken by the camera was digitized to an 8-bit gray value image (256 intensity levels) and saved as TIFF-file. To calculate the shadowgraph intensity $S(\vec{x})$ at a certain temperature difference $\Delta T$, the intensity of the raw image $I(\vec{x}, \Delta T)$ was divided by a background image $I_{0}(\vec{x})$ taken at $\Delta T=0 \mathrm{~K}$. In this way, one eliminates additional modulation effects by inhomogeneous illumination or reflection of the bottom plate, for example, or other imperfections in the optics. In order to get the shadowgraph modulation $(S(\vec{x})$ from Eqn. 3.2) one has to subtract 1 from the normalized intensity:

$$
S(\vec{x}, \Delta T)=\frac{I(\vec{x}, \Delta T)}{I_{0}(\vec{x})}-1
$$

Unfortunately, besides the shadowgraph signal produced by convection rolls, the SU-8 texture on the bottom plate surface produces an additional contribution to the shadowgraph image. During a typical convection experiment at $T_{a v}=25^{\circ} \mathrm{C}$ and $p=48.3 \mathrm{bar}$, the modulation of the index of refraction of the gas is around $\delta n / n=10^{-4}-10^{-3}$. On the other hand, the refractive index of SU-8 is $n=1.6$ [85], so the light modulation due to the surface corrugation is (with respect to the different heights) 100 times larger. ${ }^{5}$ It is not obvious how to eliminate the SU-8 effect in the shadowgraph signal for quantitative measurements. Thus, we will deal with that problem in the following paragraphs.

In the following $z_{\max }$ denotes the position at which a first maximum of the light intensity appears above cold areas in the experiment. In Fig. 3.8, shadowgraph images for $z=0, z \approx z_{\max } / 2$ and $z=z_{\max }$ are shown for the quiescent state $(\Delta T=0)$. The light is not influenced by any flow and the shadowgraph signal is solely due to the bottom plate texture. If the camera focuses on the bottom plate (left image in Fig. 3.8), refraction does not play a role and the modulation of the light is only due to a stronger absorption of light at the SU-8 ridges. If one moves the imaging plane away from the cell to a position between the cell and the first maxima, the shadowgraph effect, caused by the SU-8, comes into play, resulting in a spreading of the black areas (middle image in Fig. 3.8). The main contribution to the signal comes from the edges of the SU-8 stripes, since here the spatial derivative of the refractive index is maximal. One should note that the modulation of the intensity does not have the shape of ridges. Instead the intensity is high at the edges of the bright stripes and drops in its center. Imaging a plane at $z=z_{\max }{ }^{6}$ leads to the right hand image in Fig. 3.8. The white stripes are thin, as predicted by physical and geometrical optics.

\footnotetext{
${ }^{5}$ The refractive index of $\mathrm{CO}_{2}$ under the experimental condition is $n=1.023$.

${ }^{6}$ The position $z_{\max }$ was found qualitatively by moving the imaging plane to the position where the width of the white stripes above areas between the SU-8 ridges become minimal and their intensity maximal.
} 

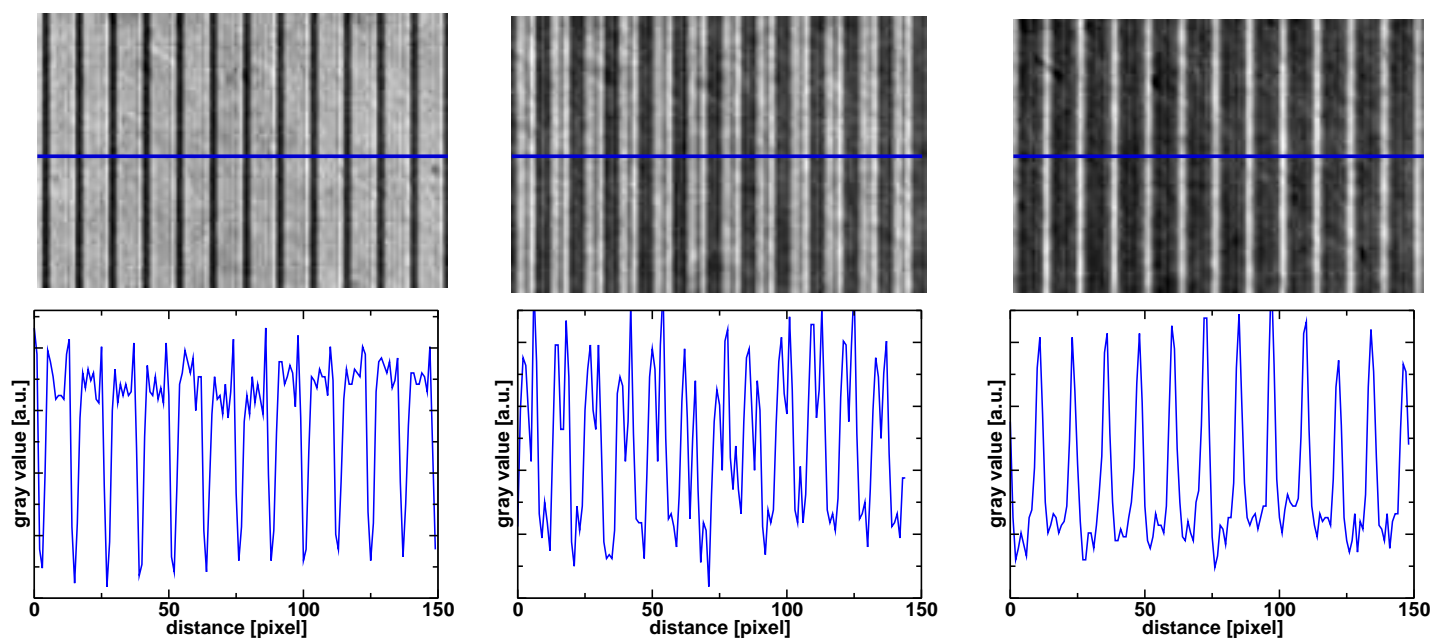

Figure 3.8: Shadowgraph intensity due to bottom plate texture. Shadowgraph images (upper row) and corresponding intensity profile plots (lower row) at $\Delta T=0 \mathrm{~K}$. Columns correspond to images of different planes in z-direction. Left: $z=0$, middle: $z \approx z_{\max } / 2$ and right: $z=z_{\max }$. Lower plots show intensity plot profile of the blue line in the images above.

The addition of light modulation due to convection rolls acts like adding more lenses to the existing SU-8- "lens array". In this way, it becomes very difficult to recalculate quantitatively the amplitude of the temperature modulation out of the shadowgraph signal and one needs to be special careful when interpreting the images of patterns. While for very weak convection, simple division by a background image is sufficient, for larger convection, a quantitative error occurs in the measurement due to this SU-8 effect, as shown in Fig. 3.9. Here, a measurement of the convection amplitude as a function of the control parameter $(\varepsilon)$ is plotted for different shadowgraph settings. As a measure for the amplitude of the convection, the standard deviation of the grayscale in each shadowgraph image is calculated and plotted in Fig. 3.9 both for the forced and the unforced case and for different shadowgraph settings. Here, we just want to compare qualitative features of the amplitude curves caused by the influence of the bottom plate texture. Quantitative experimental results on the imperfect bifurcation will be discussed in the next chapter.

As pointed out in previous chapters, it is expected that the convection amplitude for the forced and the unforced cases converge asymptotically for high $\varepsilon$. In Fig. $3.9 \mathrm{a}$, the imaged plane is very close to the convection cell. Both curves come close together for high $\varepsilon$ and although they do not converge, they end up having the same slope for high $\varepsilon$. In fact as will be shown in later chapters, the amplitude of the shadowgraph signal is not proportional to the convection amplitude but an additional term has to be added which itself is proportional to the Rayleigh 
number (see Chapter 4). In Fig. 3.9b, the shadowgraph focuses further away from the bottom plate, and while the signal of the reference is still in the linear regime (far away from the first maximum) the signal from the forced cell gets an additional amplification by the SU-8 and comes now very close to the first maxima. Here, the shadowgraph signal is no longer linear with the convection amplitude, due to the SU-8 lens effect, but increases strongly when it comes closer to the first maximum $z_{\max }$. Hence, the shadowgraph signal of the reference cell and that one of the forced cell diverge. The focus plane for the experiments shown in Fig. $3.9 \mathrm{c}$ is very close to $z_{\max }$, where the intensity signal of the pure SU-8 is almost maximal. Due to an increase in the convection amplitude the axial position were the intensity is maximal $\left(z_{\max }\right)$ is reduced and lies now below the imaged plane. The relative shadowgraph intensity increases much slower with $\varepsilon$ than the convection amplitude. While the convection amplitude increases, the conversion factor between the amplitude and the shadowgraph signal intensity decreases. Therefore, for high $\varepsilon$ the intensity signal for the forced cell is lower than for the reference cell which is not expected. For all plots shown here, a background division was done.

It is not obvious how to recalculate the convection amplitude out of the shadowgraph signal. The easiest way is to assume just a linear transformation and to introduce a correction factor $M$ which depends on the shadowgraph setting. For each setting one can choose $M$ so, that the forced amplitude curve converges for higher $\varepsilon$ with the unforced one. ${ }^{7}$ This was done in Fig. 3.9d. The curves of Fig. 3.9(b and c) are multiplied by an appropriate $M$ and do collaps on a master curve, even though they were taken with different shadowgraph settings. For quantitative experiments presented in the following chapter, the shadowgraph was focused very close to the cell. In addition, $M$ was considered as an additional fit parameter during fitting of a cubic amplitude equation.

The effect of the bottom plate structure on the shadowgraph signal can be seen, if one increases the convection amplitude to values, so that $z_{\max }$ crosses the imaging plane with increasing $\varepsilon\left(z<z_{\max } \longrightarrow z>z_{\max }\right)$. The corresponding amplitude plot is shown in Fig. 3.10. Representative shadowgraph images are shown in Fig. 3.11. For small $\varepsilon$ the shadowgraph amplitude is as expected and at $\varepsilon=0$ convection sets in, in the reference cell. But the shadowgraph signal of the forced convection and that one of the unforced convection do not converge asymptotically but the forced convection amplitude reaches a maxima at around $\varepsilon=0.23$ and decreases again, while the amplitude of the unforced cell still increases. Here, in the shadowgraph image of the forced cell, thin bright

\footnotetext{
${ }^{7}$ Not that this is only true for small forcing parameter $\delta$ and small $\varepsilon$, as predicted by theory. However one can assume, that the relative influence of forcing increases with increasing $\varepsilon$ and the behavior becomes more and more similar to the unforced case. From this point of view one can expect at least the same slope for the forced and unforced amplitude curves at high $\varepsilon$. In any case, this paragraph should motivate the additional fit parameter $M$.
} 

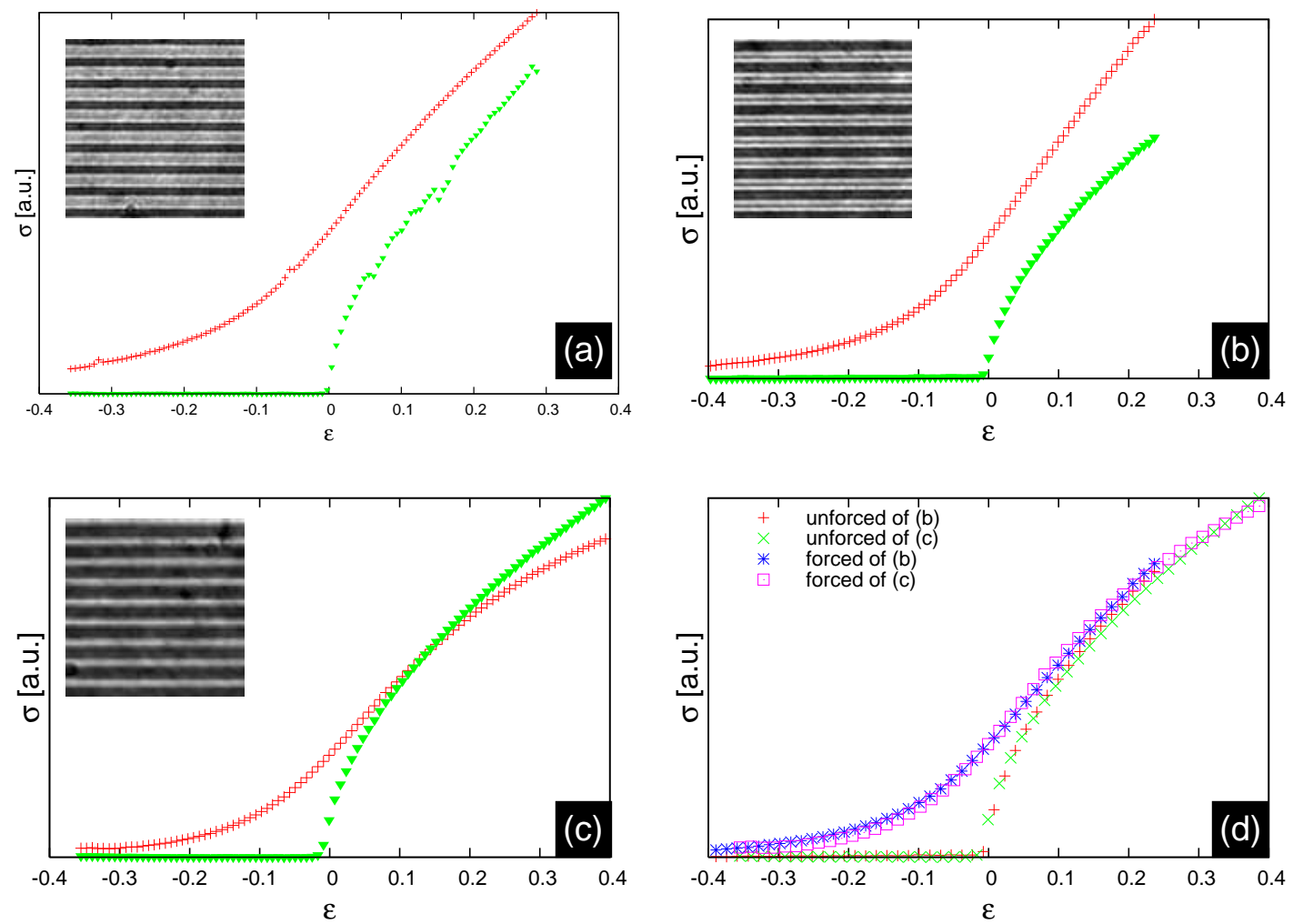

Figure 3.9: Standard deviation of the intensity for reference cell $(\boldsymbol{\nabla})$ and forced cell $(+)$, for different shadowgraph settings. Focusing close to the bottom plate (a), between bottom plate and first maximum (b) and at the first maximum (c). The inlets show a small section of the corresponding background image. (d): Via a linear transformation plots (b) and (c) sit on the same master curve.

stripes occur at the dark area between the wide white stripes. In the reference cell the stripes just become first thinner and later on in a similar manner thin white stripe also occur here at former dark areas (at around $\varepsilon=0.3$ ). The dark areas become a bit less dark in this way and the bright become a bit less bright. The standard deviation of the shadowgraph signal of the forced and the unforced case decrease until in both cases a minimum is reached at $\varepsilon \approx 0.8$ (forced convection) and $\varepsilon \approx 1.3$ (unforced convection). With increasing $\varepsilon$ the intensity of the small white stripes which appeared first in the dark regions, increases as well and finally become brighter as the original white stripes. Therefore, the standard deviation of the shadowgraph images increases again to reach a second maximum. The curve for the reference cell becomes messy at $\varepsilon \approx 1.65$ since here spiral defect chaos sets in. 


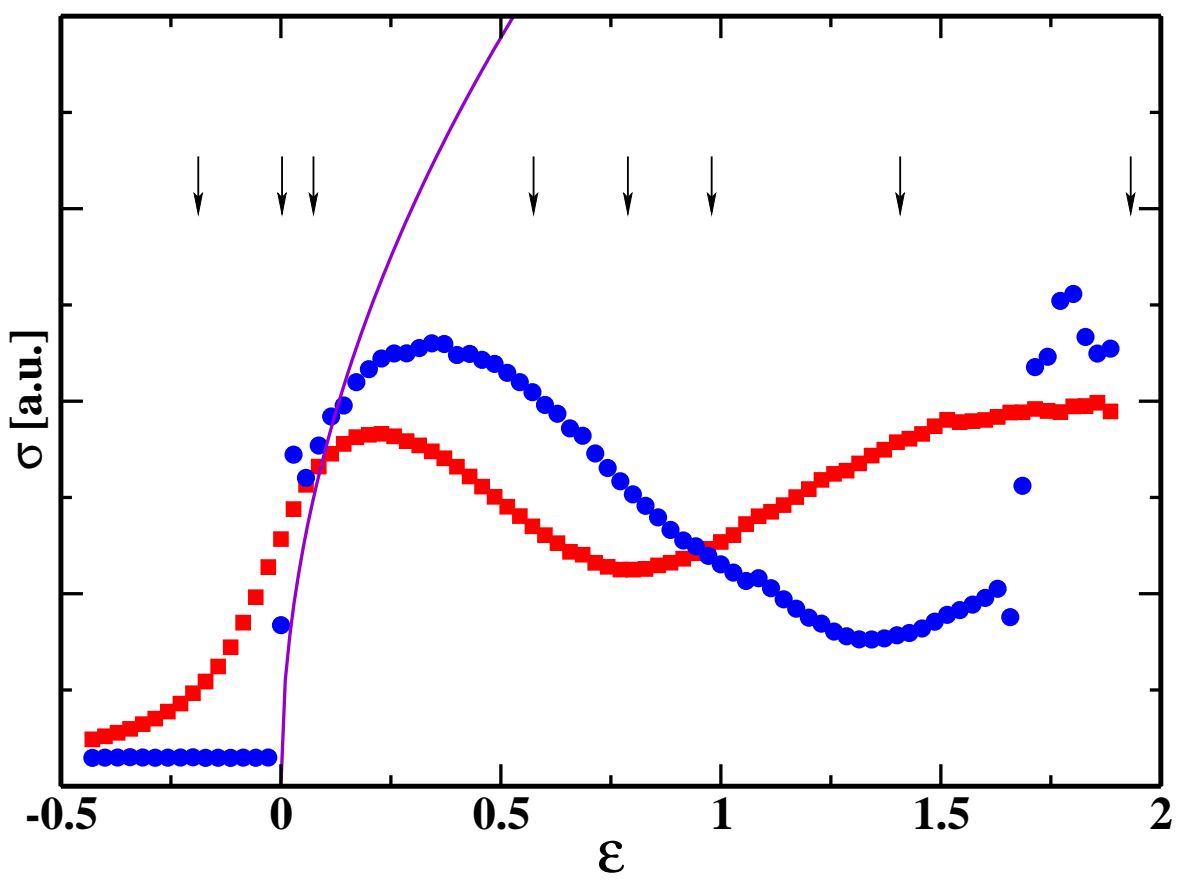

Figure 3.10: Standard deviation of the shadowgraph signal for forced (red squares) and reference cell (blue circles) in dependence on the control parameter $\varepsilon$. The image plane is at approx $z \approx 2 \cdot z_{\max }$. The violet solid line is a fit for the reference cell data. Small arrows mark the positions of the representative images for Fig. 3.11.

\subsubsection{Some Remarks about Fourier Analysis}

In this work a large variety of different patterns found in forced thermal convection is presented. Mostly the shape of certain patterns were of interest but not the exact temperature distribution in the cell. In order to analyze a specific pattern Fourier transformation is an extremely helpful tool. Fourier transformation was done via a Fast Hartley Transform $(\mathrm{FHT})^{8}$ as implemented in the image processing software ImageJ [86].

Fourier spectra shown in this thesis represent the modulus of the Fourier transform $|F(\vec{q})|$. It is often necessary know the amplitude of the periodic temperature modulation in a specific direction. This amplitude corresponds in general with

${ }^{8} \mathrm{~A}$ Hartley transform is very similar to a Fourier transform, but it transforms real functions to real functions by using $\sin ()$ and $\cos ()$. 

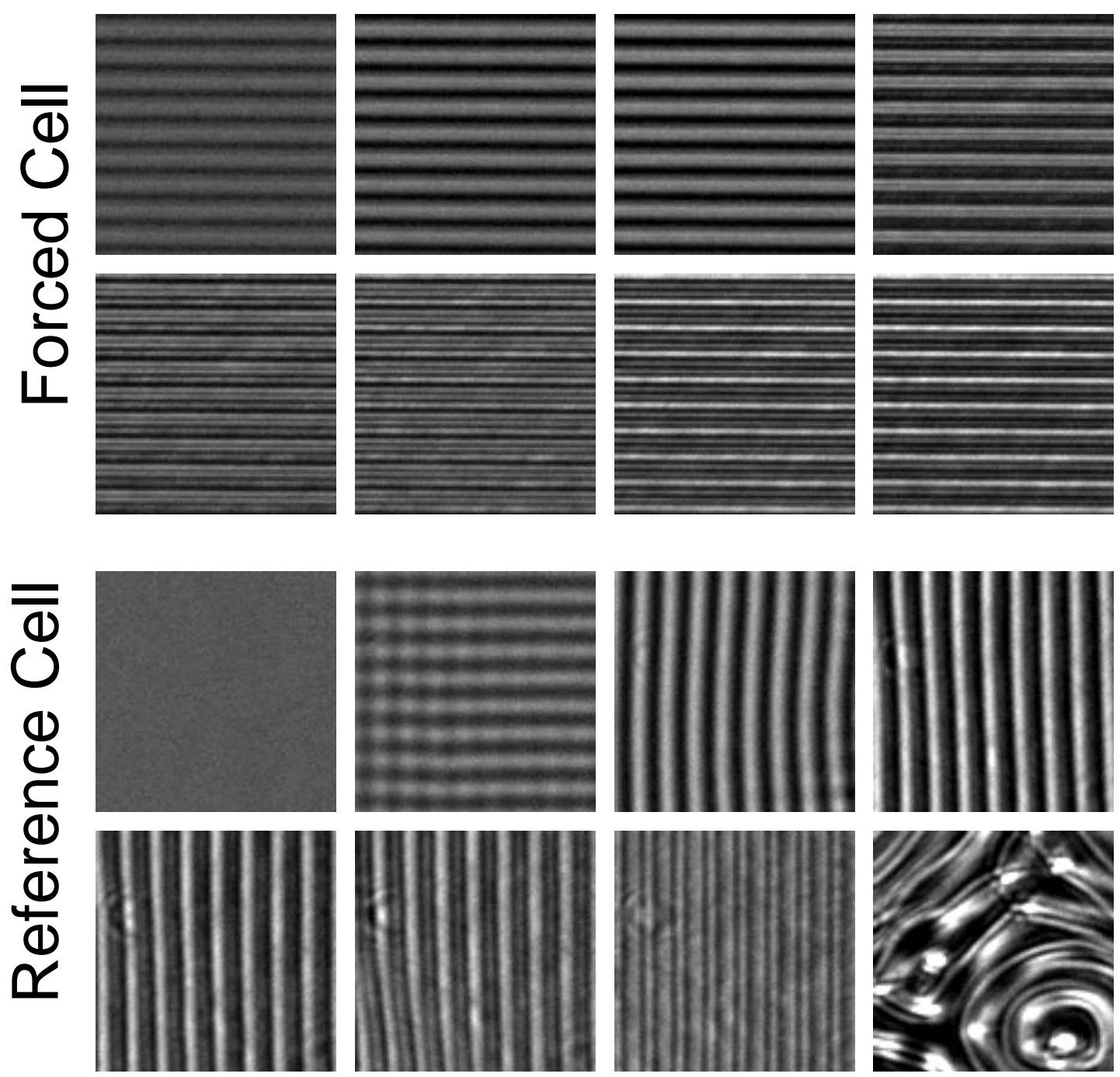

Figure 3.11: Shadowgraph images of the forced and the reference cell taken at: $\varepsilon=$ $-0.143 ; 0.001 ; 0.057 ; 0.572 ; 0.800 ; 0.971 ; 1.371 ; 1.886$ (from top left to bottom right). The position of each $\varepsilon$ is shown by arrows in Fig. 3.10. 
the height of a certain peak in the Fourier space. However, the shape of a peak for the forcing mode is very sharp and strongly localized, while peaks of side modes are usually smeared out. The temperature modulation in forcing direction is usually phase locked with the underlying SU-8 structure and does not have any defects. This is not the case for side modes. There, the defects in phase and amplitude lower the peak height in the Fourier spectra and increase their width.

An example is shown in Fig. 3.12. Here a bimodal pattern is considered, taken at an inclination angle $\gamma=75^{\circ}$ and $\varepsilon=0.13$. In the Fourier spectra (Fig. 3.12b) one sees clearly the forced mode (red square) and an additional second side mode (blue square). One can decompose the original image into the two modes, by using a mask for the Fourier transform where only an area around a peak is taken and the rest of the Fourier transform is set to zero. Afterwards, one does an inverse Fourier transformation and gets a real space image where only the chosen mode exists. In this way Fig. 3.12(c and d) were calculated. One sees clearly that in (c), where only the forced mode was used, the stripes are free of defects and phase locked with the SU-8. In comparison, the stripe pattern calculated by using only the second (unforced) mode (Fig. 3.12d) shows phase defects (green encircled), localized amplitude modulations (yellow) and in addition the stripes are also not perfect straight but their phase shows slow random modulations in space.

All these effects result in a broadened peak for the unforced mode. In Fig. 3.12(e and f) 3D plots of the forced and unforced peaks are shown. One should note the different scales used for the z-axis in the plots (maximal values at the axis are $14 \cdot 10^{5}$ in (e) and $9 \cdot 10^{5}$ in (f) in arbitrary units). This broadening effect makes it very difficult to compare the development of both peaks with each other. For a bimodal pattern one can write the equation for the temperature modulation and hence the modulation of the shadowgraph intensity:

$$
I=A_{1} \cos \left(q_{1} x\right)+A_{2} \sin \left(q_{2} y\right) .
$$

Here, one would like to know the evolution of $A_{1}$ and $A_{2}$ in dependence of the control parameter or time. What one can do is to consider the standard deviation of the original image for each row to get a measure for $A_{1}$ and if one calculates the standard deviation for each column, one gets $A_{2}$. The method works well if the modes are aligned with the rows and the columns of the pixels, but becomes complicated if they are aligned in other directions. Already for a bimodal pattern as in Fig. 3.12a this approach fails for the amplitude of the forced mode $\left(A_{2}\right)$, since the unforced modulation is not perfectly aligned with the columns of the image (as shown in Fig. 3.12b).

If both modes would be equally blurred it would not matter whether one measures the height of a peak (maximal gray value), if one measures the integral over a peak or if one just measures the standard deviation of an area around the peak. The peak height $(\max )$, its mean average intensity (mean) and its standard deviation 

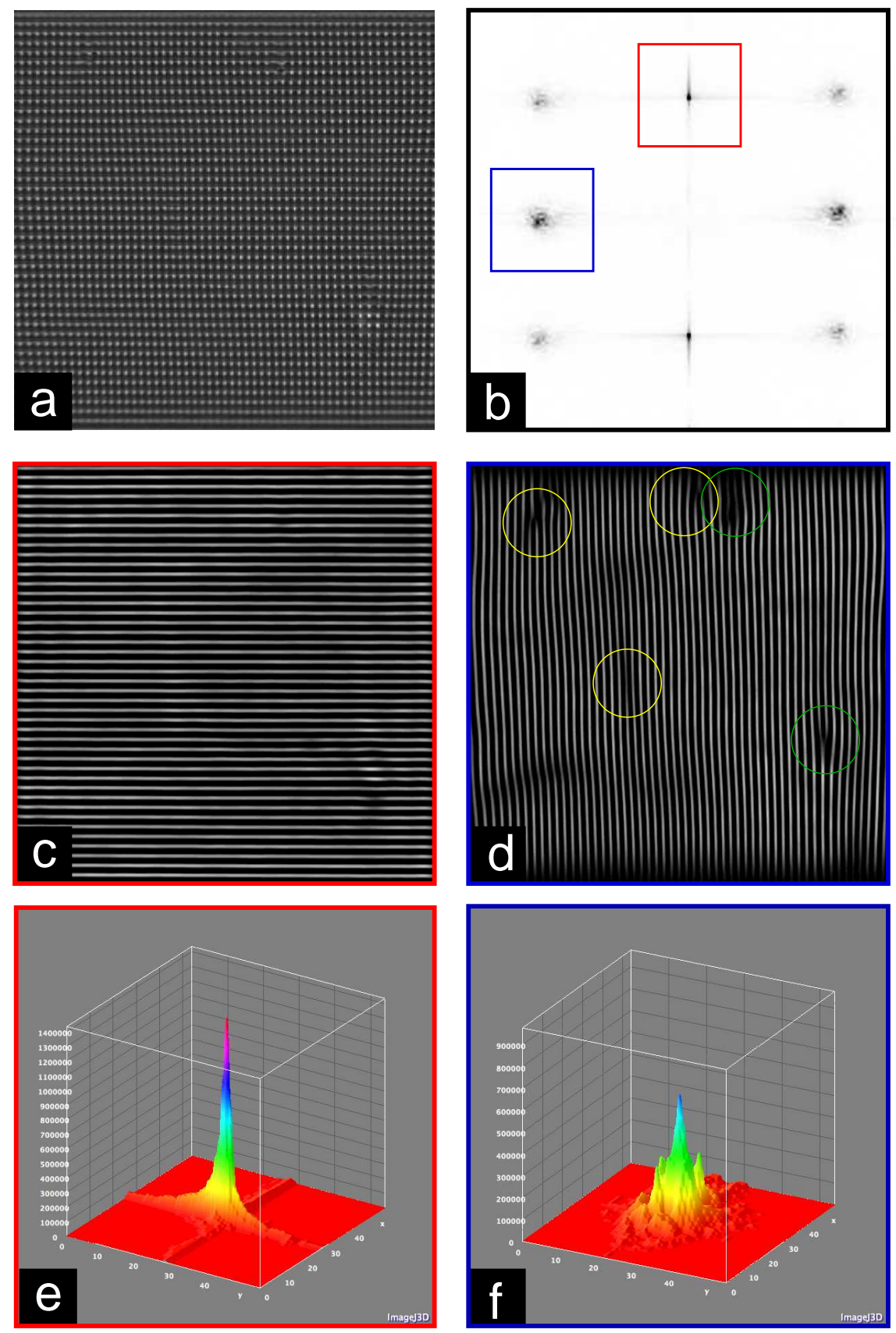

Figure 3.12: Consideration of the amplitude of specific modes. As an example a bimodal pattern was chosen (a). In (b) the Fourier spectra is shown. The forced mode and the second unforced mode are marked with a red and a blue square. (c) and (d) show decompositions of (a) where in (c) everything but the forced mode was erased in the Fourier spectra and later on transformed back to real space. For (d) everything but the unforced mode was erased. Amplitude and phase defects are marked with yellow and green circles. In the lower row 3D plots of the forced (e) and the unforced peaks (f) are shown. 
(std) are listed in table 3.1 for the forced mode peak $A_{2}$ and the side peak $A_{1}$. To calculate these values a threshold was first set in order not to count very small fluctuations of the intensity. As expected, the peak maximum is for the forced peak two times higher than for the side peak. But surprisingly the mean intensity of the side peak is 1.67 times larger than for the forced peak. If one considers the standard deviation one gets a ratio between the forced peak and the side peak of 0.96 .

Another way to measure the amplitudes of the modes is to calculate the standard deviation of the Fourier filtered image of Fig. 3.12(c and d). The resulting values are shown in the last column of Table 3.1. The ratio $A_{1} / A_{2}$ as calculated in this way gives a value of 0.98 , which is very close to the value if one takes the standard deviation of an area around a peak (third column in Table 3.1). Also in a simple simulation were a image was generated with a sharp mode and another blurred mode, the standard deviation of an area around a Fourier peak gives the best result. For this simulation, equation 3.4 was used and white noise was added in the phase of the mode in $y$-direction.

\begin{tabular}{|c|c|c|c|c|}
\hline & $\max [$ a.u.] & mean [a.u.] & std [a.u.] & std after filtering [a.u.] \\
\hline \hline$A_{1}$ & 12.16 & 0.15 & 0.54 & 2929 \\
\hline$A_{2}$ & 24.00 & 0.09 & 0.56 & 2980 \\
\hline$A_{1} / A_{2}$ & 0.51 & 1.67 & 0.96 & 0.98 \\
\hline
\end{tabular}

Table 3.1: Characteristic values of Fourier peaks.

Apparently the standard deviation around a peak is the best measure for the actual amplitude of a specific mode. One would expect that the standard deviation decreases with the peak height, but this is partially counterbalanced by the mountain like structure at the tails of the peak. To get quantitative results on the amplitude development of certain modes the Fourier transform was calculated, and a threshold filter was used in order to suppress small amplitudes in the Fourier space. Than the standard deviation of an area around the first order mode was calculated and used as a measure of its amplitude.

\subsubsection{Phase Demodulation}

It is often interesting for the analysis of certain patterns to know the underlying phase field. The shadowgraph signal itself can be considered as the real part $\Re(\psi)$ of a complex field $\psi(x, y)=|\psi(x, y)| \exp (\mathrm{i} \phi(x, y))$, with modulus $|\psi|$ and phase $\phi$. To reconstruct $\psi$, one first Fourier transforms the original pattern and sets the complex conjugated points to zero. The inverse Fourier transform now gives a complex pattern with a modulus and a phase. 
This procedure was done for a simple stripe pattern in Fig. 3.13a. The pattern is a forced thermal convection stripe pattern, phase locked to the forcing stripes of the bottom plate (taken at $\gamma=0^{\circ}$ and $\varepsilon=2.2$ ). Its Fourier transform (Fig. $3.13 \mathrm{~b})$ shows two very sharp peaks at $\left(0, \pm q_{f}\right)$ with a high amplitude (red circle). The corresponding phase field of the stripe pattern is shown in Fig. 3.13c. The value of the phase goes from $-\pi$ (black) to $\pi$ (white).
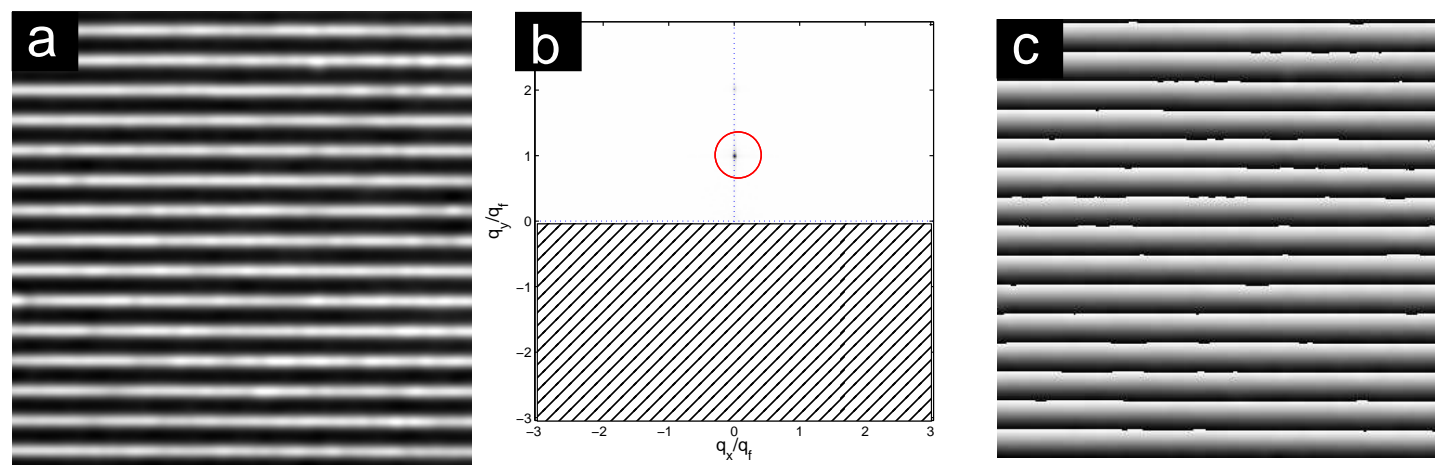

Figure 3.13: Stripe pattern (a), its Fourier spectra (b) and its phase field (c). The mode in (b) is encircled red, since the peak is very sharp due to the straight defect-free stripe pattern.

For a simple stripe pattern as in Fig. 3.13 the phase is a linear function of the space:

$$
\phi(x, y)=x q_{x}+y q_{y}
$$

with $q_{x}=0$ and $q_{y}=q_{f}$, regarding Fig. 3.13. For more complicated patterns, the phase itself can be modulated in an arbitrary manner:

$$
\phi(x, y)=x q_{x}+y q_{y}+\beta(x, y)
$$

The modulation of the phase field $\beta(x, y)$ can be calculated by subtracting a background phase field - the phase of a forced striped pattern (Fig. 3.13c) - from the modulated phase field $\phi$. This is shown in Fig. 3.14 for the Varicose Pattern, which will be described in detail in the next chapter (Sec. 4.2.1).

Fig. 3.14a shows the Varicose pattern at $\gamma=0^{\circ}$ and $\varepsilon=2.58$. The corresponding phase field is shown in Fig. 3.14b. Figure 3.14c shows the modulation part of the phase field $\beta$, i.e. the difference between the phase field of the varicose pattern and the phase field of convection rolls with the forcing wave number $q_{f}$.

In the following whenever a phase field is mentioned, only the modulated part $\beta(x, y)$ is meant. 

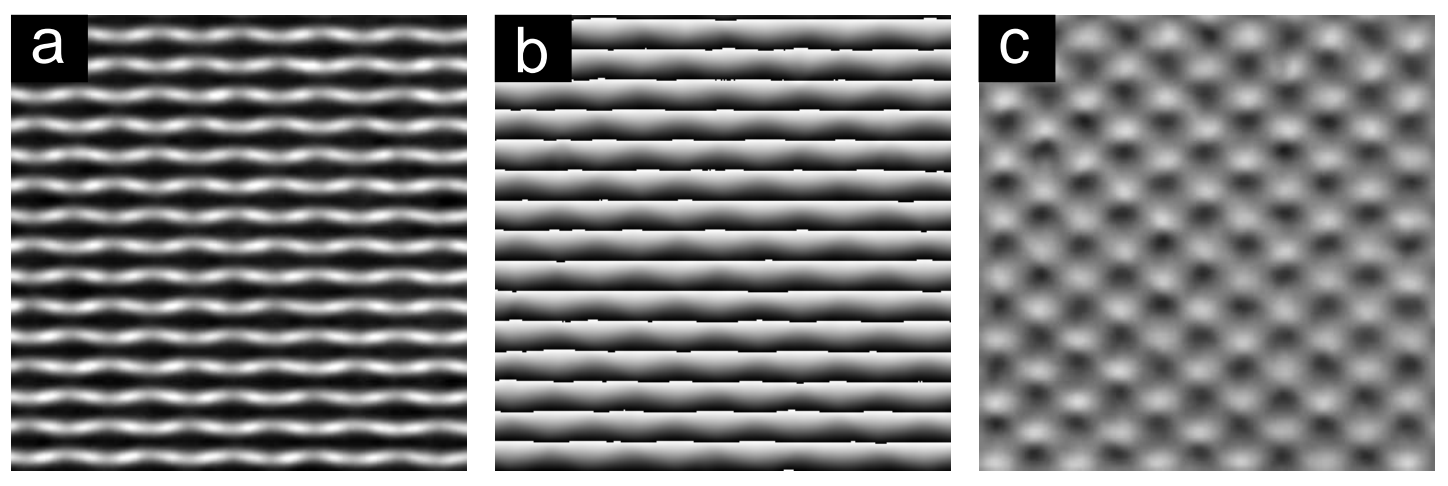

Figure 3.14: Varicose Pattern (a), its phase field (b) and only its modulated part (c). The measurement was done at $\gamma=0^{\circ}$ and $\varepsilon=2.58$. 


\section{Chapter 4}

\section{Inclined and Noninclined Resonant Forcing}

In this chapter we consider the case of resonant forcing with a corrugation wave number close to the critical wave number of the unforced system $\left(q_{f} \approx q_{c}\right)$. In addition, the system can be inclined giving a second symmetry breaking mechanism.

The relative orientation of both is given by $\varphi$, the angle between the gravity and the SU-8 ridges (see Fig. 4.1). By changing the inclination angle $\gamma$ and the temperature difference $\Delta T$, one is able to tune the relative strength. Several results presented in this chapter are already published in [87].

The chapter consists of four sections. Section 4.1 considers the amplitude of straight forced rolls without inclination. The other sections report experiments and analysis on forced inclined layer convection with angles $\varphi=0^{\circ}$ (Sec. 4.2), 90 (Sec. 4.3), and $\varphi=60^{\circ}$ (Sec. 4.4).

\subsection{Forced Rolls and Amplitude Equation}

Here, we describe the evolution of the amplitude of the forced rolls. Instabilities are considered in the next sections. In fact, this is a repetition of a previous work by McCoy [68]. However, here $q_{f}$ is closer to $q_{c}\left(q_{f}=12 / 11 q_{c}\right.$, instead of $q_{f}=6 / 5 q_{c}$ in [68]) and in addition, in this work the shadowgraph effect of the underlying SU-8 texture is taken into consideration by the additional fit parameter $M$ as introduced in Sec. 3.3.2.

As described in Sec. 2.2, due to harmonic forcing $\left(q_{f} \approx q_{c}\right)$ an additional constant term is expected to appear in the amplitude equation (Eq. 2.25). The amplitude of the forced convection rolls follows therefore an imperfect bifurcation instead of a pitchfork bifurcation as in the unforced case (see e.g., [88] and [15] ). This 


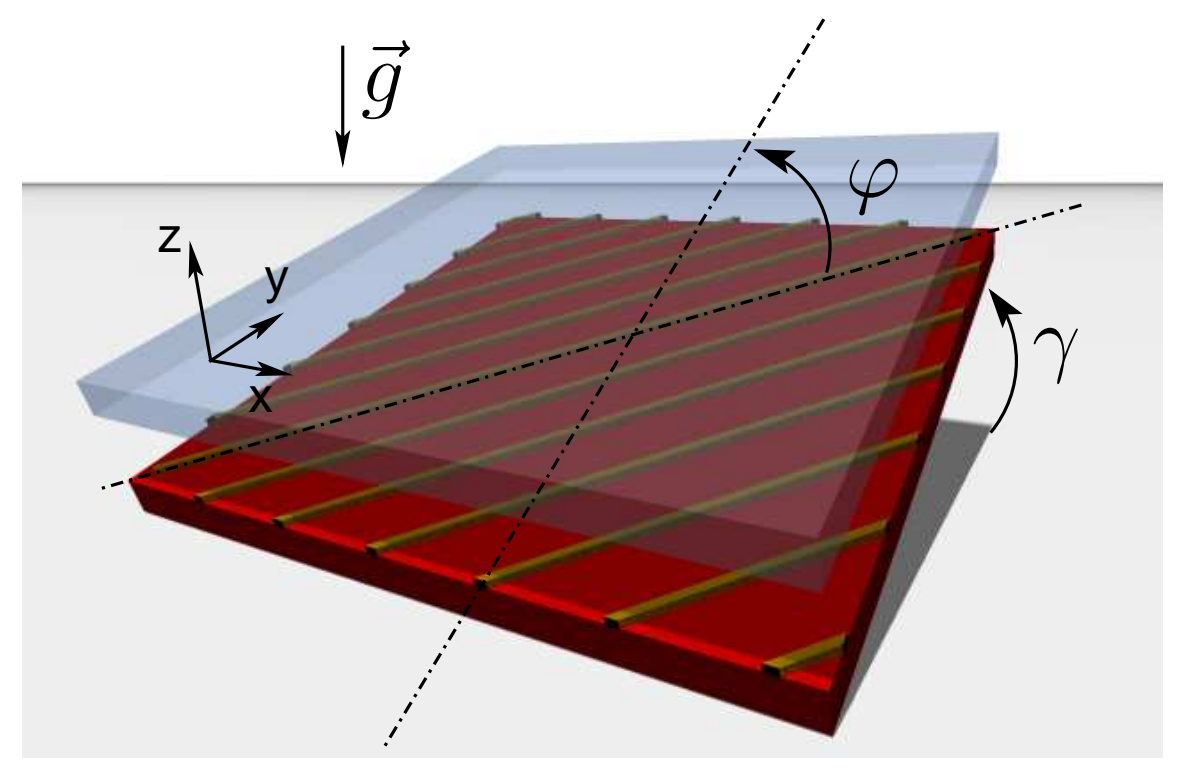

Figure 4.1: Schematic of the convection cell for forced ILC. The cell can be inclined in two independent directions corresponding to two independent parameters: The inclination angle $\gamma$ and the angle between the SU- 8 structure and the in-plane gravity component $\varphi$.

means that even for very small temperature differences $(\varepsilon<0)$ convection occurs. We consider Eq. 2.25, which describes the amplitude of convection rolls as a function of the Rayleigh number $R a$. Equation 2.25 was derived via weakly nonlinear theory and is valid for $R a$ close to $R a_{0}\left(q_{f}\right)$, where $R a_{0}\left(q_{f}\right)$ is the Rayleigh number at the neutral curve for a specific $q_{f}$ (see Sec. 2.2). For forcing wave numbers $\left(q_{f}\right)$ close to, but not exactly equal to the critical wave number of the system $\left(q_{f} \approx q_{c}\right)$ one can approximate the neutral curve as a parabola (as shown e.g., in [16]):

$$
R a_{0}\left(q_{f}\right) \approx R a_{c}\left(1+\xi_{0}^{2}\left(q_{f}-q_{c}\right)^{2}\right) \quad \text { with } \quad \xi_{0}^{2}=0.1479
$$

Including the expression for $R a_{0}$ into the amplitude equation (Eq. 2.25) together with the control parameter $\varepsilon=\left(R a-R a_{c}\right) / R a_{c}$ one gets:

$$
\left.\left[\varepsilon-\xi_{0}^{2}\left(q_{f}-q_{c}\right)^{2}\right)\right] A-g_{0} A^{3}+g_{2} \delta=0 .
$$

This equation represents a imperfect bifurcation, that is shifted on the $\varepsilon$-axis to the right by $\left.\xi_{0}^{2}\left(q_{f}-q_{c}\right)^{2}\right)$. As explained in the previous chapter (Eq. 2.26 and 2.27) The values of the coefficient are:

$$
g_{0}=4.4823 \cdot 10^{-6} \text { and } g_{2}=238 \text {. }
$$


Since the cell height for this experiment was set to $520 \mu \mathrm{m}$ and the periodicity of the SU- 8 stripes is $1 \mathrm{~mm}$, the forcing wave number is $q_{f}=(12 / 11) q_{c}$. In order to verify Eq. 4.1 one first has to consider the relation between the shadowgraph amplitude $A_{s}$ and the actual convection amplitude $A$. The shadowgraph signal $S(x, y)$ of the forced cell can be written as [68]:

$$
S(x, y)=G\left(\frac{q_{f}}{q_{c}}\right)^{2} \int_{-1 / 2}^{1 / 2}\left[T_{\text {mod }}(x, z)+\frac{\Delta T}{R a} \theta(x, y, z)\right] .
$$

$G$ is an unknown conversion parameter which depends only on the optical system. It includes the setting of the shadowgraph, the light intensity and the camera settings.

For small amplitude convection an equation for the shadowgraph amplitude $A_{s}$ can be derived (see [68] for a rigorous derivation):

$$
A_{s}=G^{\prime}\left(\frac{q_{f}}{q_{c}}\right)^{2}\left[\left(\delta \frac{\cosh \left(q_{f}\right)-\cosh (0)}{2 q_{f} \sinh \left(q_{f}\right)}\right) R a+\left\langle g_{1}\right\rangle_{z} A\right] .
$$

All constants are absorbed by $G^{\prime} .\left\langle g_{1}\right\rangle_{z}$ is the z-dependent part of the solution of the linearized Boussinesq equation ${ }^{1}$, averaged over the cell height, it is $\left\langle g_{1}\right\rangle_{z}=$ $0.611[68]$.

While in the unforced case, the shadowgraph amplitude is proportional to the convection amplitude $\left(A_{s}=G^{\prime}\left\langle g_{1}\right\rangle_{z} A\right)$, here $A_{s}$ contains also an additional constant term that is independent of $A$. This constant term exist, due to the temperature splitting (Eq. 2.13) into a linear part $\left(T_{\text {lin }}\right)$, a modulated part $\left(T_{\text {mod }}\right)$ that fulfills the boundary conditions and the heat equation, and in a convective part $(\theta)$. One can say, that even without a convective fluid motion $(\vec{v}=0, \theta=0)$, a temperature modulation would exists due to the SU-8 texture and therefore an additional signal is produced in the shadowgraph even for $A=0$.

In order to determine $G^{\prime}$, measurements from the unforced reference cell are used. The amplitude equation for the shadowgraph signal there is:

$$
\varepsilon A_{s}-g_{0} \frac{A_{s}^{3}}{G^{\prime 2}\left\langle g_{1}\right\rangle_{z}^{2}}=0
$$

One can fit the curve $A_{s}=\left(G^{\prime}\left\langle g_{1}\right\rangle_{z}\right) \cdot \sqrt{\frac{\varepsilon}{g_{0}}}$ and gets in this way $G^{\prime}$, which is then used for the fit of the forced convection amplitude.

Amplitude measurements for forced and unforced convection without inclination are shown in Fig. 4.2. The blue circles show the shadowgraph amplitude in the

\footnotetext{
${ }^{1} \mathrm{~A}$ solution of the linearized Boussinesq equation can be written as: $\theta_{1}(x, z)=g_{1}(z)\left[A_{1} \exp \left(\mathrm{i} q_{f} x\right)+c . c.\right]$
} 
reference cell as a function of the control parameter $\varepsilon$. Following a pitchfork bifurcation the amplitude is zero for negative $\varepsilon$ and increases according to a square root law for positive $\varepsilon$. The solid blue line is a fit and gives the value for $G^{\prime}$, which is then used to calculate the amplitude $A$ in the forced cell.

To compensate the influence of the SU-8 texture on the shadowgraph signal, the measured amplitude of the forcing rolls was multiplied by a factor $M$ (see Chapter 3). This linear correction is the simplest possible one and assumes, that the measured shadowgraph $\left(A_{s}^{m}\right)$ amplitude contains an additional term, which results from the bottom plate texture and that is proportional to the theoretical convection amplitude $\left(A_{s}\right)$ :

$$
A_{s}^{m}=A_{s}+\Gamma A_{s}=(1+\Gamma) A_{s}=M \cdot A_{s}
$$

Here $\Gamma$ is only a proportionality factor for the additional correction, but will not be used in the following, since it is absorbed into $M$, which is used as an additional fit parameter.

Let us now calculate the value of $\delta$ based on Eq. 2.9. $\delta$ is given by the ratio of the height of the bottom plate texture and the cell height $(\mathrm{h} / \mathrm{d}=65 \mu \mathrm{m} / 520 \mu \mathrm{m})$. Since the texture has a step-like profile instead of a sinusoidal one as assumed in theory, the profile has to be Fourier approximated to first order. Together with the first Fourier coefficient $a_{1}=0.196$ the forcing parameter is:

$$
\delta_{\text {exp }}=\frac{a_{1} h}{d}=0.0245 .
$$

A theoretically predicted curve, following Eq. 4.1 and with $\delta=\delta_{\exp }$ is plotted in Fig. 4.2 (green). The red triangles in Fig. 4.2 show the convection amplitude in the forced cell. The experimental points follow qualitatively the theoretical curve, even though the experimental values are always larger.

In order to compare quantitatively the experimental results with the theory, all parameters, but the forcing parameter $\delta$ were held constant and $\delta$ and the correction parameter $M$ were fitted. For the fit, only values $-0.05<\varepsilon<0.05$ were used, since Eq. 4.1 is only valid for small $\varepsilon$. The resulting value is:

$$
\delta_{\text {fit }}=0.0322 \text { and } M=0.90
$$

Both values $\left(\delta_{f i g}\right.$ and $\left.\delta_{\text {exp }}\right)$ are of the same order, but differ by $25 \%$. There are three possible explanation for the discrepancy. First, the perturbation theory leading to Eq. 4.1 is only valid for small $\delta$ and $\varepsilon \approx 0$, i.e., the expansion parameter $\mu=\delta^{1 / 3} \ll 0$ (introduced in Eq. 2.21) has to be much smaller than one. With $\delta \approx 0.03$ the corresponding $\mu=0.31$, which cannot be considered $\ll 1$. As can be seen in Fig. 4.2 theory and experiment come closer for $\varepsilon \approx 0$ and differ more for larger and smaller $\varepsilon$. 


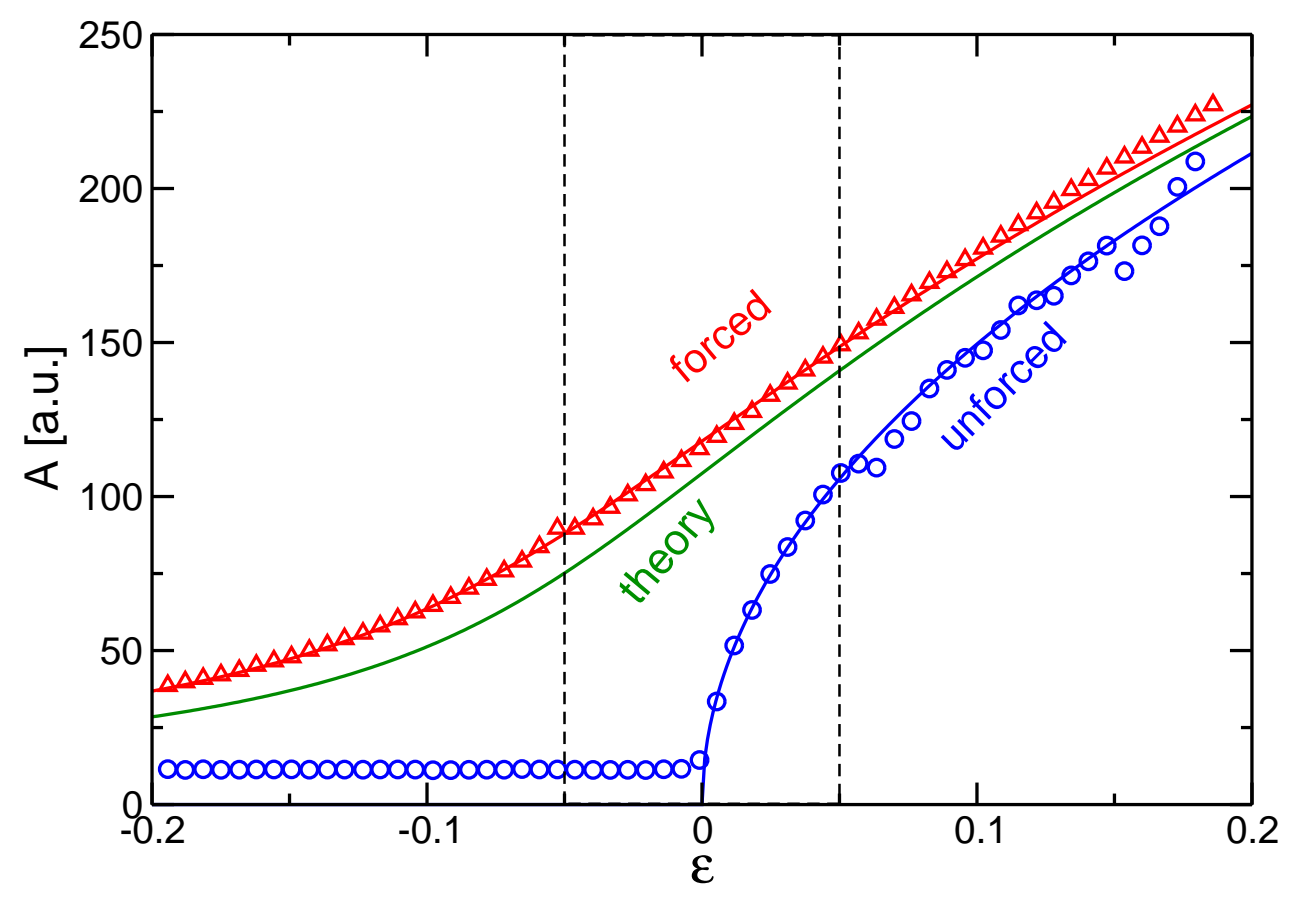

Figure 4.2: Measured amplitude as a function of $\varepsilon$ for the forced $(\triangle)$ and the unforced cell $(\mathrm{O})$. The blue and red solid lines mark the corresponding fitted curves. The solid green line shows the theoretical prediction. For the fit, only the values between the two black dashed lines were used.

Second, the periodic bottom plate corrugation, which has the form of a step function was approximated only by the first Fourier term, a harmonic function with wave number $q_{f}$. And as a third reason, there is still the nonlinear dependency between shadowgraph amplitude and the real convection amplitude, which cannot be completely compensated by introducing the factor $M$. In spite of all these assumptions a good qualitative agreement was found.

\subsection{Forcing with a Wave Vector Perpendicular to Gravity - Longitudinal Forcing}

In unforced inclined layer convection (ILC), longitudinal rolls, aligned with their axis parallel to the gravity component, occur at convection onset for inclination 
angles $\gamma<\gamma_{c d}$. For the experiments presented here, the Prandtl number is $\operatorname{Pr}=1.27$ and the codimension-two point is located at $\gamma_{c d} \approx 80^{\circ}$, as shown in Fig. 2.2. In this section, experiments are reported for which $\varphi=0^{\circ}$ was chosen (see Fig. 4.1), i.e., the bottom plate forcing supports the development of longitudinal rolls and therefore fosters for $\gamma<\gamma_{c d}$ the natural (unforced) pattern selection process (longitudinal forcing).

Figure 4.3 summarizes the experimental results. Different symbols mark different observed patterns. The solid black lines are guides to the eye and delimitate boundaries between different patterns. Each pattern will be discussed in detail in the following subsections. Note that the critical temperature difference for the convection onset without forcing increases with increasing inclination angle. This limited the experiments for $\theta>80^{\circ}$ to $\varepsilon \approx 0.2$ since the maximal possible temperature difference of $\Delta T \approx 11 K$ of the experiment was reached. ${ }^{2}$

The control parameter $\varepsilon$ is defined as the relative distance above the convection onset in the unforced reference cell $\Delta T_{c}^{3}$ :

$$
\varepsilon=\frac{\Delta T-\Delta T_{c}}{\Delta T_{c}}
$$

For inclination angles $\gamma<80^{\circ}$, straight longitudinal rolls (LR) bifurcate in an imperfect bifurcation. The occurring rolls are defect free and perfectly aligned with the forcing pattern (Fig. 4.4). Secondary instabilities of forced LR occur for $\gamma<72^{\circ}$ at larger $\varepsilon$ than for unforced LR (dashed line in Fig. 4.3) [48]. One can therefore conclude, that resonant forcing of longitudinal rolls in an ILC experiment stabilizes the natively occurring longitudinal rolls.

Somehow unexpected is the secondary instability for high inclination. For inclination angles close to the codimension-two point $\left(73^{\circ}<\gamma<82^{\circ}\right)$ the longitudinal rolls become unstable at $\varepsilon=0$ to transverse bursts (TB), while for even higher inclination angle, the longitudinal rolls are stable up to $\varepsilon=0.04$ and then turn to transverse rolls (TR). Why should the forced longitudinal rolls be more stable for higher inclination angles? This might be due to a strong coupling of the forcing mode $\vec{q}_{f}$ and a second side mode that together build up the transverse bursts (see Sec. 4.2.3).

Let us now consider the primary instabilities, when the forced longitudinal rolls become unstable and bifurcate into new spatio-temporal patterns. These patterns will be explained in detail in the following. Starting with the horizontal case $(\theta=0)$, the pattern at the right of the phase diagram will be explained first,

\footnotetext{
${ }^{2}$ For higher $T_{a v}$ and small modifications of the system, the maximal temperature difference between bottom and top plate could be increased to $15 \mathrm{~K}$. However, for these conditions the Boussinesq approximation can not be considered to be valid anymore.

${ }^{3}$ Note the difference to $\varepsilon$ in Fig. 2.2. $\frac{\Delta T-\Delta T_{c}}{\Delta T_{c}} \neq \frac{R a-1708}{1708}$. Both definitions are equal for $\gamma<\gamma_{c d}$.
} 

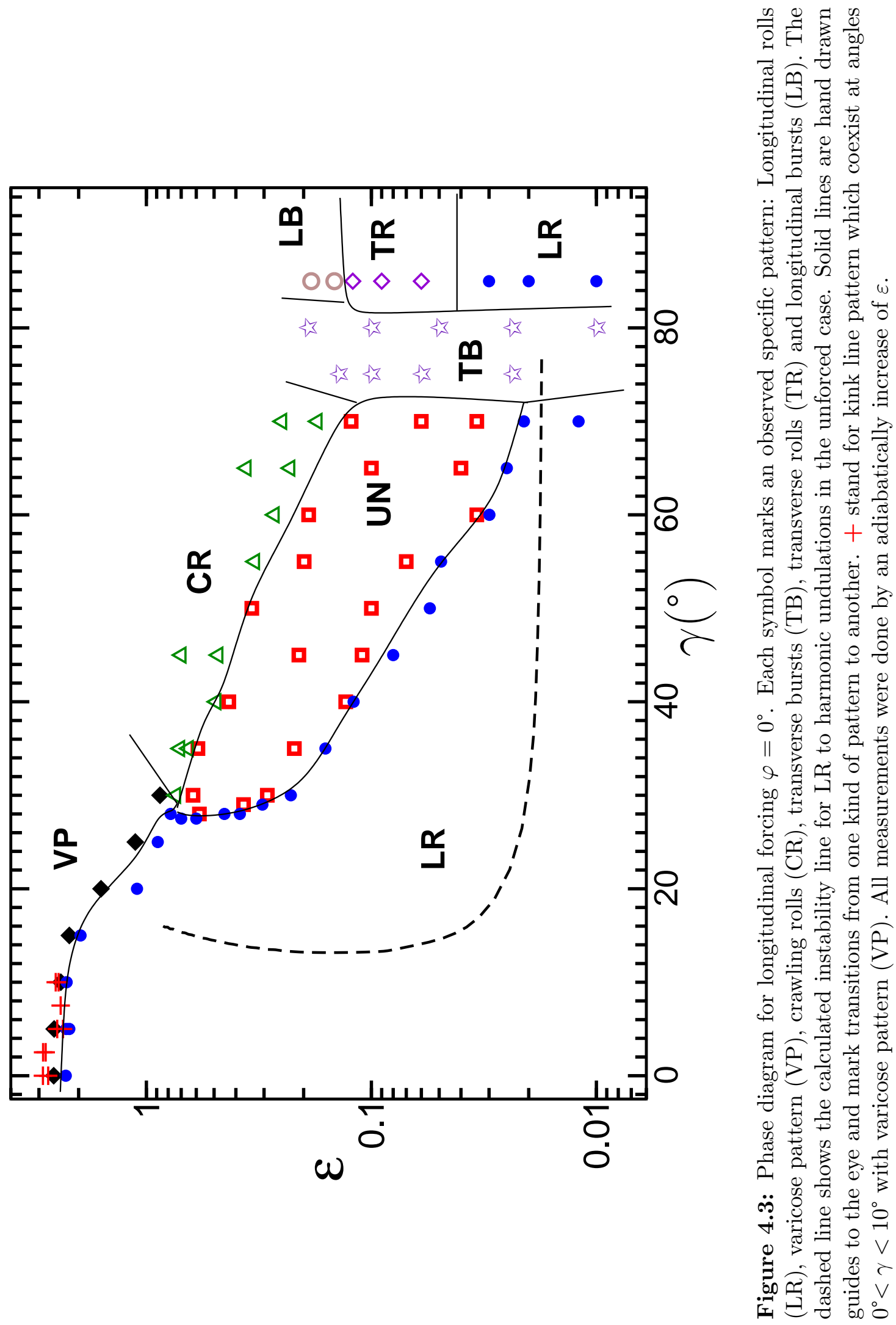


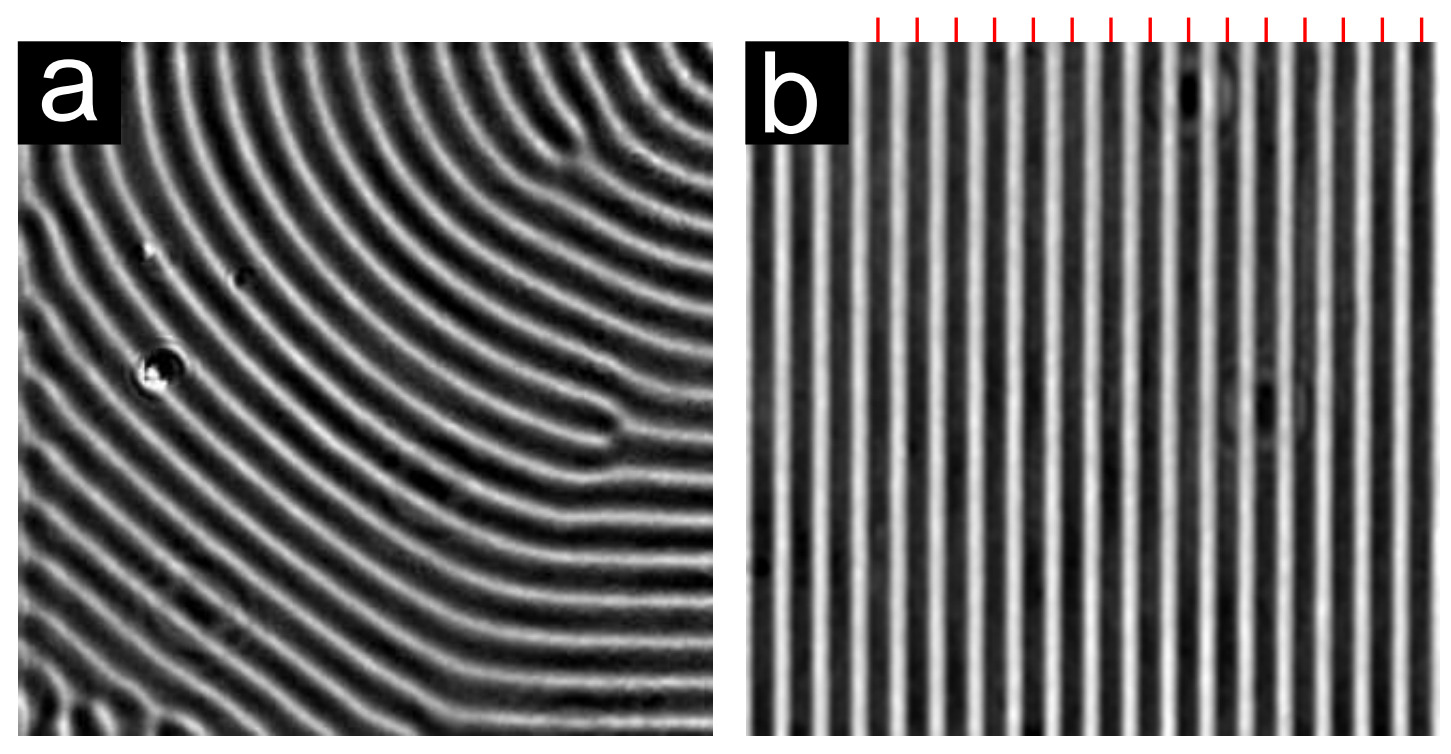

Figure 4.4: Straight rolls in unforced (a) and forced (b) convection experiments. Both pictures were taken without inclination at $\varepsilon=0.16$ and show a squared section of the cell of a width $28 d$. While in the unforced cell, the convection rolls are curved in order to be aligned perpendicular to the boundaries, the forced convection rolls are perfectly aligned with the underlying bottom plate texture (red stripes at the top).

followed by the moderate and large inclination angle patterns. At the end of the section we will consider several different phenomena that occur at very small inclination angles, such as kinks ( + in 4.3 ) and others that are not explicitly labeled in the phase diagram. All images that are shown in this section are aligned with the gravity, so that the in-plane gravity component $\left(\vec{g}_{\|}\right)$points from up to down. In addition, in most of the images the location of the bottom plate texture is marked with red lines.

\subsubsection{Varicose Pattern}

For $\gamma=0^{\circ}$ straight rolls were unstable at $\varepsilon \approx 2.4$ to a varicose pattern (VP) as shown in Fig. 4.5. Here, the rolls bent periodically in a subharmonic way, meaning the phase modulation of neighboring rolls have opposite signs and thus the modulation of each roll is in-phase again with the next but one roll. The Fourier spectrum in Fig. 4.5 reveals the modes. In addition to the forced mode $\vec{q}_{f}=\left(q_{f}, 0\right)$, two new modes occur which are symmetric regarding the x-axis and build an angle of $36^{\circ}$ with the forcing wave vector $\vec{q}_{f}$ :

$$
\vec{q}_{1,2} \approx q_{f}\left(\begin{array}{c}
0.5 \\
\pm 0.35
\end{array}\right) \approx 0.62 q_{f}\left(\begin{array}{c}
\cos \left(36^{\circ}\right) \\
\pm \sin \left(36^{\circ}\right)
\end{array}\right)
$$



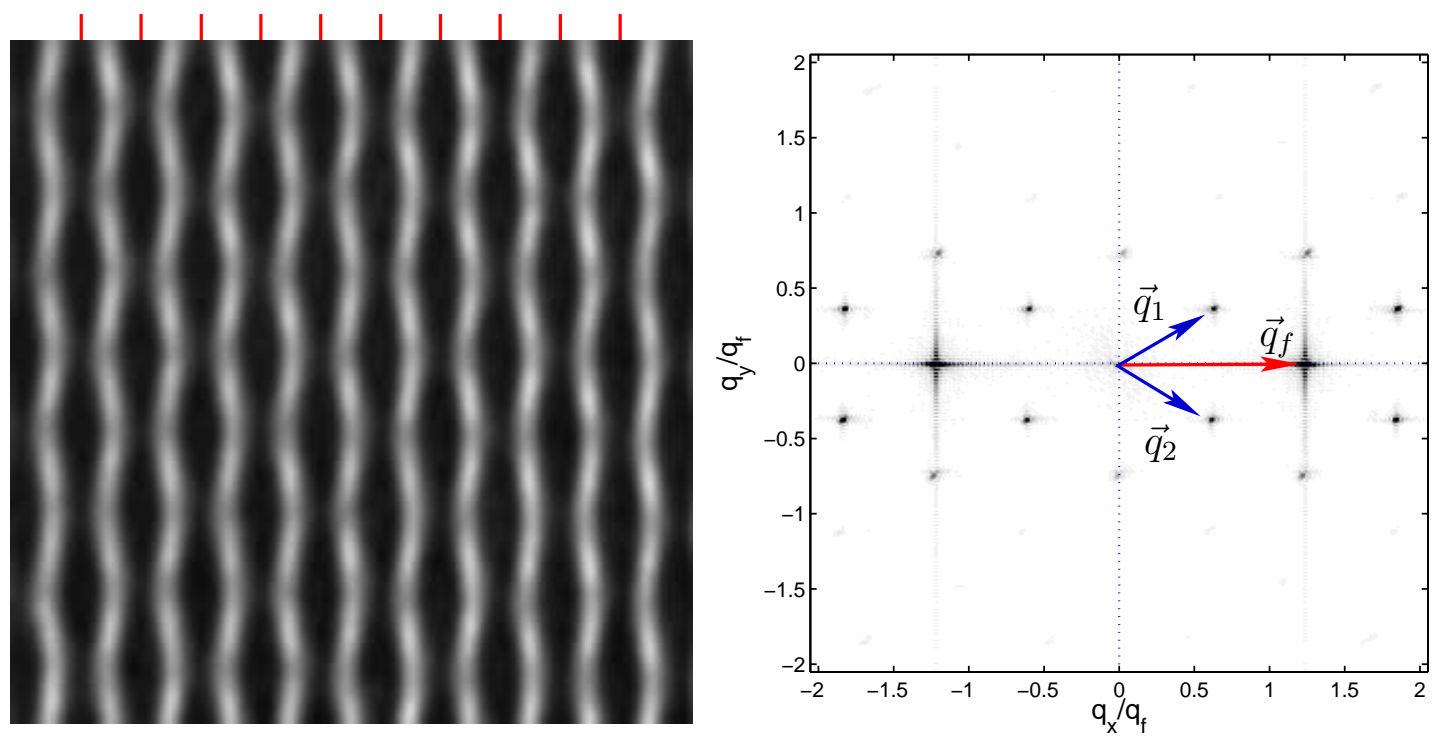

Figure 4.5: Varicose pattern (left) and the central part of its Fourier transform (right). The image was taken for $\gamma=0^{\circ}$ and $\varepsilon=2.7$. The red lines in the left image mark the locations of the forcing ridges.

These three modes fulfill the resonant triade condition:

$$
\overrightarrow{q_{f}}=\overrightarrow{q_{1}}+\overrightarrow{q_{2}}
$$

In fact, the modes modulate the phase of the rolls in a periodic way similar to a skewed varicose instability but with two symmetric wave vectors instead of only one. Such a pattern can be described mathematically by following formula:

$$
I_{V P}(\vec{r})=A_{0} \cos \left(\vec{q}_{f} \vec{r}+A_{1}\left[\cos \left(\vec{q}_{1} \vec{r}\right)+\cos \left(\overrightarrow{q_{2}} \vec{r}\right)\right]\right) .
$$

An intensity distribution calculated from Eq. 4.4 is shown in Fig. 4.6. Here, the following amplitudes were chosen: $A_{0}=1$ and $A_{1}=0.3$.

In Fig. 4.7 the modulated part of the phase regarding Eq. $4.4\left(A_{1}\left(\cos \left(\overrightarrow{q_{1}} \vec{r}\right)+\right.\right.$ $\left.\left.\cos \left(\vec{q}_{2} \vec{r}\right)\right)\right)$ and the calculated phase field from an experiment are compared. The image in Fig. 4.7b is a false color version of Fig. 3.14c taken at $\gamma=0^{\circ}$ and $\varepsilon=2.58$.

The varicose pattern is steady in time, defect free and stable up to $\varepsilon \approx 2.7$.

Neither the length nor the direction of $\vec{q}_{1}$ and $\vec{q}_{2}$ changes significantly when $\varepsilon$ is increased. Only the strength of the phase modulation, expressed by the amplitude $A_{1}$, grows with increasing $\varepsilon$ which manifests in a stronger wiggling. At $\varepsilon \approx 2.7$ the wiggling is so strong that two rolls come close enough to merge and produce a 

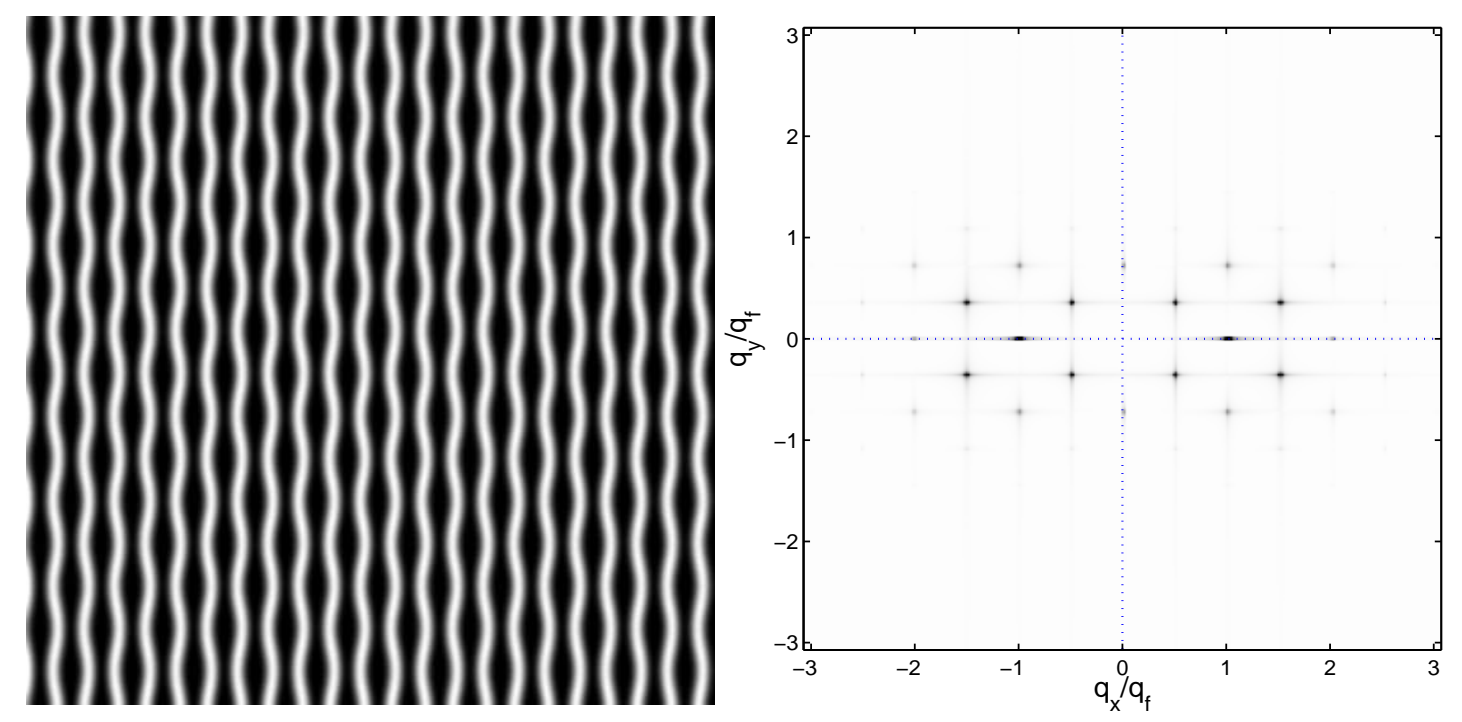

Figure 4.6: Calculated varicose pattern regarding Eq. 4.4 (left) and the central part of its Fourier transform (right). The amplitudes here are $A_{0}=1$ and $A_{1}=0.3$.
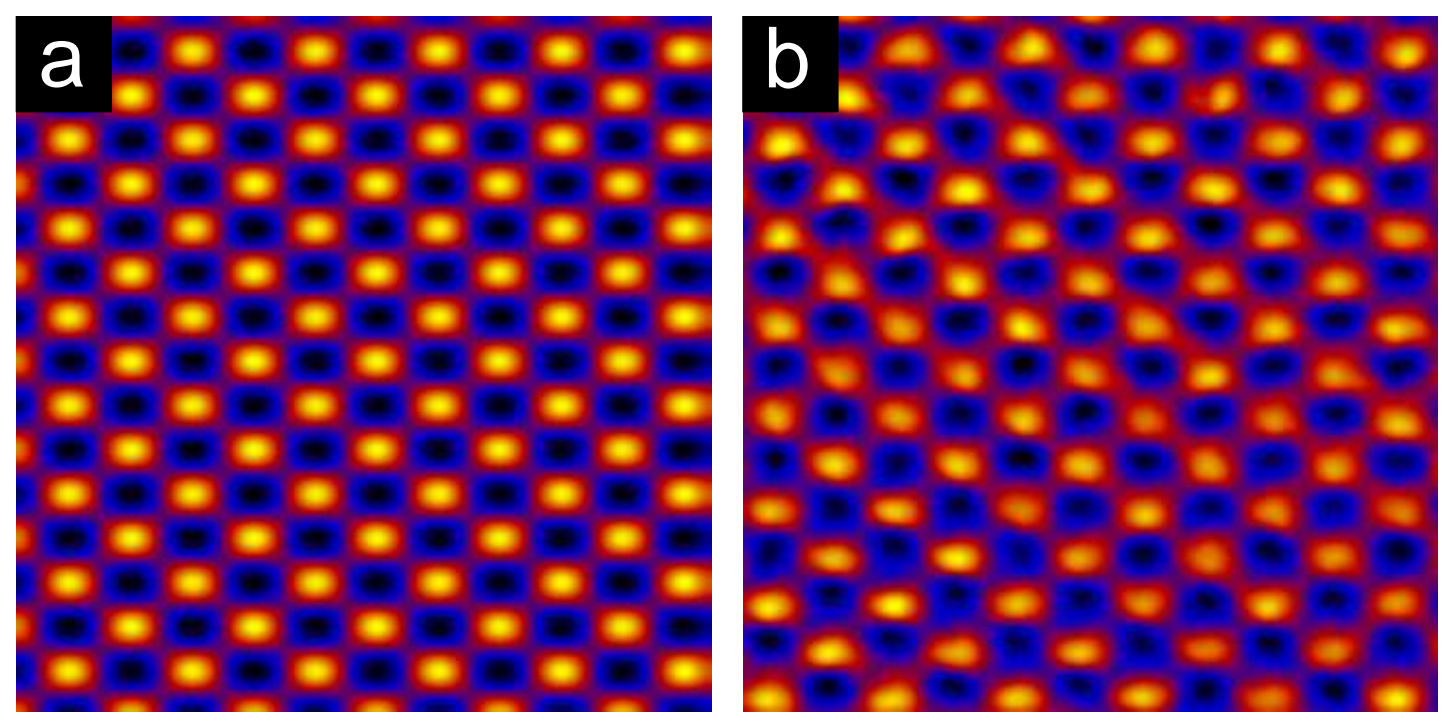

Figure 4.7: Modulation of the phase field of varicose pattern. (a) shows the calculated phase field of Eq. 4.4: $A_{1}\left(\cos \left(\overrightarrow{q_{1}} \vec{r}\right)+\cos \left(\vec{q}_{2} \vec{r}\right)\right)$ with $A_{1}=0.3$. (b) is the phase field calculated from the experimental VP at $\gamma=0^{\circ}$ and $\varepsilon=2.58$. 
defect. Immediately the pattern turns everywhere in a highly mobile and spatiotemporal chaotic state as shown in Fig. 4.8 (movie available on enclosed CDROM). The same chaotic state was observed by McCoy [68] already for much smaller control parameter values $(\varepsilon>1.4)$ and for larger forcing wave number $\left(q_{f}=6 / 5 q_{c}\right.$ in his case and $q_{f}=12 / 11 q_{c}$ here). In his experiments he also found varicose pattern, but only as a transient state while increasing $\varepsilon$ very fast. For a slow adiabatic increase of $\varepsilon$ he found localized phase kink defects instead, that travel through the cell and that can either cluster together or annihilate each other [68, 69]. Similar kinks were also found in forced inclined layer convection and are described later in this thesis. The influence of a change in the forcing wave number on the pattern selection process in horizontal convection is investigated in Chapter 5.
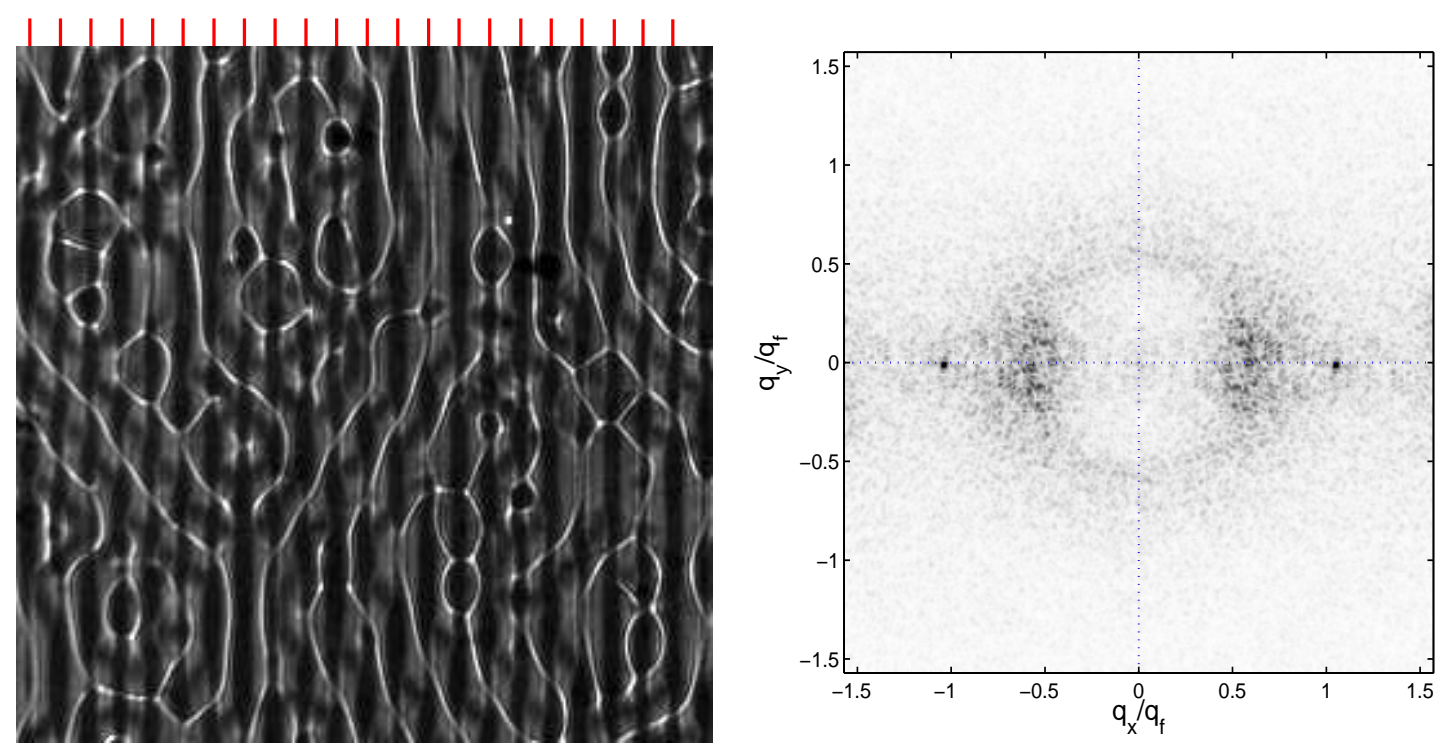

Figure 4.8: Spatio-temporal chaotic state for horizontal convection (left) and its corresponding Fourier transform (right). The experiment was done at $\varepsilon=2.65$. The red lines mark the location of the bottom plate texture. Note that the shadowgraph was (unintentionally) set here to image a plane very close to $z_{\max }$. Therefore the occurring white (cold) areas appear as as very thin lines.

VP existed not only for the horizontal case but did also occur for inclination angles up to $\gamma=28^{\circ}$. Neither the magnitude nor the angle of $\vec{q}_{1}$ and $\overrightarrow{q_{2}}$ change significantly with increasing inclination angle. While for small inclinations $\left(\gamma<7.5^{\circ}\right)$ $\mathrm{VP}$ are steady, for increasing angle, i.e., shear flow, VP drift in downhill direction (e.g. $v_{\text {drift }}=0.53 d / \tau_{\kappa}$ at $\gamma=10^{\circ}$ and $\varepsilon=2.31$ ). From their morphology, VP are similar to subharmonic oscillations found in experiments and theory at similar parameter range for unforced ILC [48, 51]. From this point of view, VP are a stabilized version of subharmonic oscillations. 
While VP existed over a broad range of inclination angles, for $2.5^{\circ}<\gamma<10^{\circ}$ this state coexists with other interesting phenomena (' + ' in phase diagram Fig. 4.3), such as coherent kink lines, subharmonic oscillations or crawling rolls. All these patterns will be explained in a later section.

\subsubsection{Harmonic Undulation and Crawling Rolls}

In unforced ILC at inclination angles $\gamma>12^{\circ}$ an instability occurs which is of the zig-zag type. As discussed in [47], this zig-zag instability saturates at one point and a so called wavy pattern, or undulation (UN) survives. The theoretically predicted instability of longitudinal rolls to undulations is shown as a dashed line in the phase diagram in Fig. 4.3. The experimentally observed instability curve [48] agreed well with the theoretical prediction. However, in the experiment a competition and bistability between ordered undulations and undulation chaos was found. Applying forcing stabilizes the longitudinal rolls against the zigzag instability significantly and the onset occurs at much higher $\varepsilon$ and larger inclination angles $\gamma$ than in the unforced case (' $\square$ ' in Fig. 4.3).
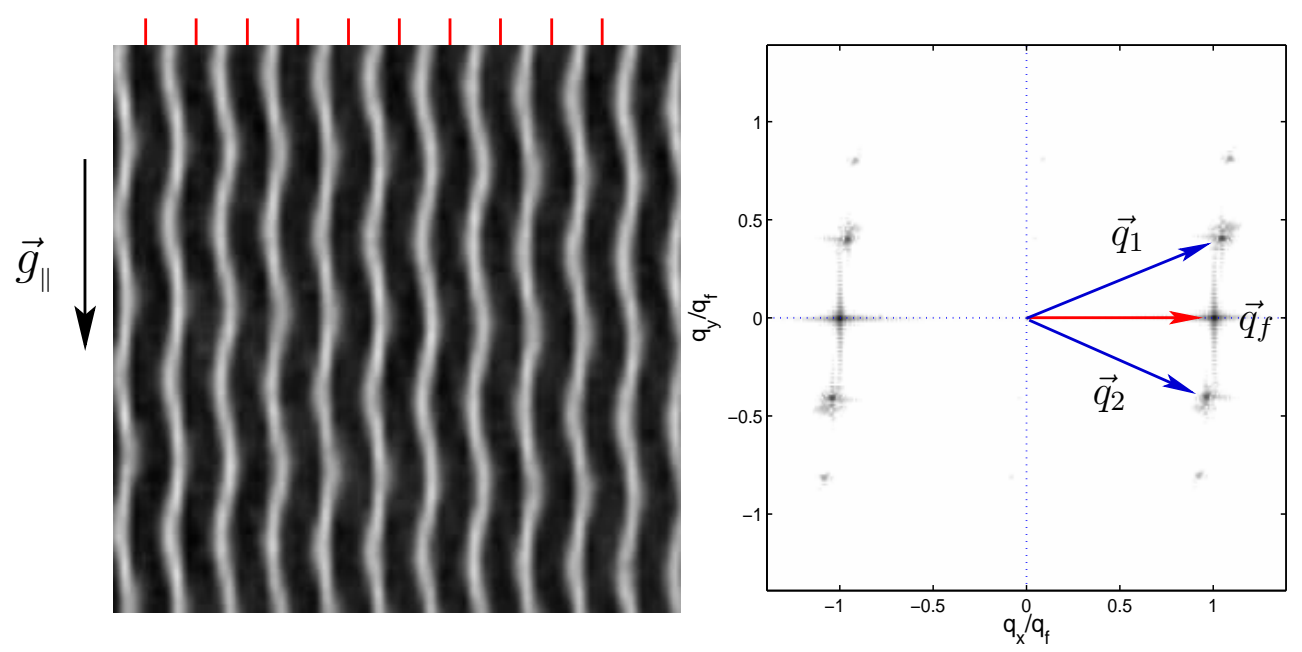

Figure 4.9: Harmonic undulations (left) and their corresponding Fourier spectrum (right) for $\gamma=55^{\circ}$ and $\varepsilon=0.28$. The red lines mark the locations of the forcing ridges.

Figure 4.9 shows a shadowgraph image of the forced undulations and the center of its Fourier transform. In contrast to undulation chaos in unforced ILC $^{4}$, undulations in forced ILC are nearly defect free and show only a slight uphill drift with increasing speed for larger shear flow (movie available on enclosed CD-ROM).

\footnotetext{
${ }^{4}$ Unforced undulations often switches between a more ordered state and a defect turbulent state as shown in [50].
} 
The drift velocity ranges from $v_{\text {drift }} \approx 0.07 \lambda_{f} / t_{\kappa}\left(\right.$ at $\gamma=50^{\circ}$ and $\varepsilon=0.1$ ) to $v_{\text {drift }} \approx 0.4 \lambda_{f} / t_{\kappa}\left(\right.$ at $\gamma=65^{\circ}$ and $\left.\varepsilon=0.1\right)$.

In the Fourier transform of the forced undulation pattern in Fig. $4.9 \mathrm{~b}$ one can identify three different modes, which are the forcing wave vector $\vec{q}_{f}=\left(q_{f}, 0\right)$ and the instability modes $\vec{q}_{1}=\left(q_{f}, q_{y}\right)$ and $\overrightarrow{q_{2}}=\left(q_{f},-q_{y}\right)$.

These modes resonate with each other:

$$
2 \vec{q}_{f}=\vec{q}_{1}+\vec{q}_{2}
$$

As measured in Fig. 4.9, for $\gamma=55^{\circ}$ and $\varepsilon=0.28$, the specific values are:

$$
\vec{q}_{1,2} \approx q_{f}\left(\begin{array}{c}
1 \\
\pm 0.42
\end{array}\right) \approx 1.07 q_{f}\left(\begin{array}{c}
\cos \left(23^{\circ}\right) \\
\pm \sin \left(23^{\circ}\right)
\end{array}\right)
$$

In general, the y-component of $\vec{q}_{1}$ and $\vec{q}_{2}$ depends on the inclination angle and varies from $q_{y}=0.47 q_{f}$ at $\gamma=30^{\circ}$ to $q_{y}=0.24$ at $\gamma=65^{\circ}$, as measured at onset of undulations. Often, $\vec{q}_{1}$ and $\vec{q}_{2}$ are not fully symmetric but slightly tilted. This might be attributed to a weak large scale circular flow in the horizontal plane due to slightly inhomogeneities in the lateral boundaries.

A clearer indication of a circular component of the large scale shear flow is given by the drift motion of the phase and the motion of defects. The phase field of the forced undulations is shown in Fig. 4.10b. The phase field is periodic in y-direction and shows dislocations (blue circles) and also amplitude variations (green circle). A simple PIV algorithm was used in order to determine the drift of the phase field. Fig. 4.10c shows the relative drift field of the phase, which is the difference between the drift field and a mean drift. ${ }^{5}$ While in a large region at the bottom of the picture, the phase drifts in the direction of gravity (parallel to $\vec{g}_{\|}$), at the left boundary the phase drifts in the opposite direction (antiparallel to $\vec{g}_{\|}$).

This leads to the occurrence of dislocations at the interface between the two regions (blue circle in Fig. 4.10b), that glide along the interface in y-direction (blue arrow). The gliding motion of these defects stops at maybe $2 / 3$ of the cell length and the defects move then along the stripes (climbing) in x-direction. In this analysis one discovers also a modulation of the amplitude in the upper $1 / 3$ of the cell, that drift constantly in x-direction (blue circle and blue arrow in Fig. $4.10 \mathrm{~b})$. It is expected, that these phenomena are due to a component of the large scale shear flow in $\mathrm{x}$ - and $\mathrm{y}$ - direction. ${ }^{6}$

\footnotetext{
${ }^{5}$ Therefore, the mean of the relative drift field in Fig. 4.10 is zero.

${ }^{6}$ In previous (unforced) experiments this was avoided by using a rectangular cell. However, the geometry of the textured area of the bottom plate constrains the size of the cell. A rectangular cell would thus lead to a smaller aspect ratio and the lateral boundaries would have a stronger influence on the occurring pattern.
} 

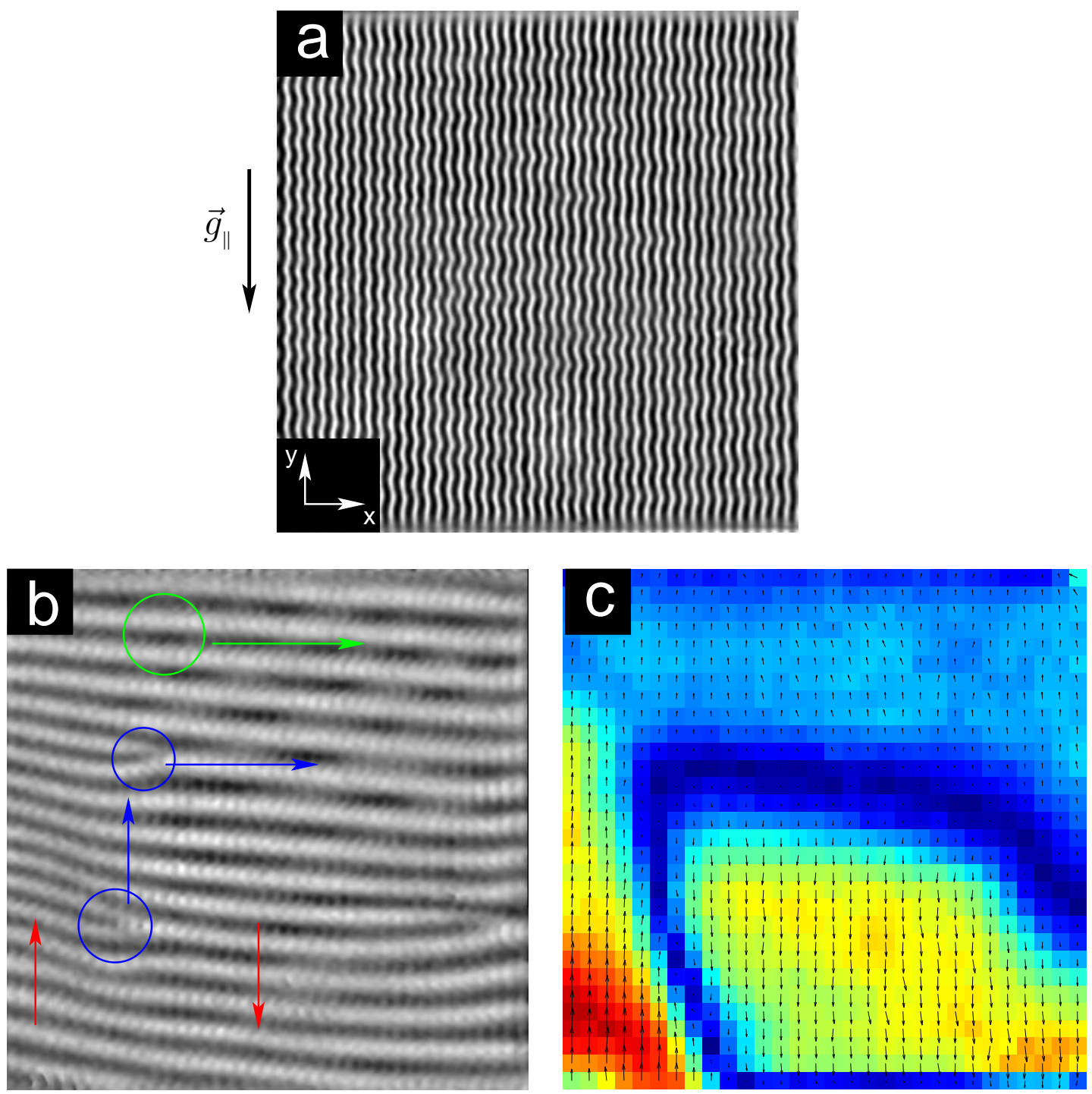

Figure 4.10: Undulation pattern (a) and its phase field (b). The red arrows mark the drift of the phase field. The blue arrows mark the motion of dislocations (blue circle) and the green arrow mark the motion of the periodic modulations of the phase field (green circle). In (c) the averaged displacement of the phase field is shown. The experiment was done at $\gamma=55^{\circ}$ and $\varepsilon=0.28$. 
Undulations are phase modulated longitudinal rolls. They can be written as:

$$
\left.I_{U N}(x, y)=\cos \left(q_{f} x+\beta(x, y)\right) \quad \text { with a periodic function } \beta(x, y)=A \sin \left(q_{y} y\right)\right) \text {. }
$$

For $A \ll 1$ one can approximate:

$$
\begin{aligned}
I_{U N}=\cos \left(q_{f} x+A \sin \left(q_{y} y\right)\right) & \approx \cos \left(q_{f} x\right)-A \sin \left(q_{f} x\right) \sin \left(q_{y} y\right) \\
& \approx \Re\left(e^{i \vec{q}_{f} \vec{r}}+\frac{A}{2}\left(e^{\mathrm{i} \vec{q}_{1} \vec{r}}-e^{-\mathrm{i} \vec{q}_{2} \vec{r}}\right)\right) .
\end{aligned}
$$

In this formula one clearly sees the existence of all three modes $\left(\vec{q}_{f}, \overrightarrow{q_{1}}, \overrightarrow{q_{2}}\right)$. This is only an approximation, since the $\sin \left(\sin \left(q_{y}\right)\right)$-term was expanded to the first order, to get to Eq. 4.6. But if one would take more terms in the expansion, one would get higher combinations of the same three modes.

In [50] a detailed analysis of undulation and undulation chaos is given for the unforced ILC and compared with theoretical simulations. It was shown that undulations are stable within a restricted area in the $q_{x}-q_{y}$ parameter space. Only vectors $\left.q_{1,2}=\overrightarrow{(} q_{x}, q_{y}\right)$ within this areas can exist. The shape and the size of this area depends strongly on $\varepsilon$, whereas for larger $\varepsilon$ the area becomes smaller and its center moves to larger values of $q_{y}{ }^{7}$. Therefore, one would expect an increase for $q_{y}$ with increasing $\varepsilon$ in the forcing experiments. While for small $\gamma$ no such behavior was observed within the measurement error of $\pm 0.015 q_{f}$, with increasing inclination $\left(\gamma>40^{\circ}\right)$ the opposite effect was observed. The wave number in y-direction became slightly smaller for increasing $\varepsilon$. For an inclination angle of $\gamma=60^{\circ}$, the value of $q_{y}$ changed from $q_{y}=0.24 q_{f}$ (at $\varepsilon=0.057$ ) to $q_{y}=0.22 q_{f}($ at $\varepsilon=0.100)$.

Beside the drift, with increasing $\varepsilon$, the phase modulation becomes more disordered. Phase and amplitude defects occur (such as shown in Fig. 4.10) and introduce more dynamic into the system. The fast traveling defects destroy more and more the order and periodicity of the system and turn it to a less ordered state, similar to undulation chaos as described in [50]. A further increase in $\varepsilon$ results finally in crawling rolls (CR) that were also observed in unforced ILC [48]. In contrast to the unforced case (see [48]), here undulation chaos is a transition state between ordered undulations and crawling rolls and therefore not explicitly marked by a symbol in the phase diagram in Fig. 4.3. The transition can also be seen in the Fourier spectra of the pattern. As shown in Fig. 4.11 the peaks for $\vec{q}_{1}$ and $\vec{q}_{2}$ get blurred and smaller modes (green circles) appear next to $\vec{q}_{1}$ and $\vec{q}_{2}$. These small modes correspond to perturbations with a long wavelength of the amplitude of the phase modulation ( $A$ in Eq. 4.5 and 4.6). These perturbations

\footnotetext{
${ }^{7}$ Note the different orientation of the co-ordinate system here and in [50].
} 
are also visible in the shadowgraph images of Fig. 4.11. Here, areas with a strong phase modulation (green rectangle in Fig. 4.11) alternate with areas of only weak phase modulation (small $A$ ). A bistability of ordered and disordered pattern was also observed in unforced ILC and discussed in detail in [50].

For a further increase in $\varepsilon$, the amplitude of the phase modulation increases while the function $\beta(x, y)$ is no longer a periodic function anymore but becomes chaotic in space and time. The small peaks in the Fourier spectra disappear and the spectra gets blurred. Even for fully developed crawling rolls, the peaks of the forcing mode is still visible.

The final state, of fully developed crawling rolls (bottom row in Fig. 4.11) has still a periodicity in $\mathrm{x}$-direction due to forcing but the phase function $\beta$ is chaotic in space and time, which cause the modes in the Fourier transform to be blurred out. Comparing this state with the crawling rolls in the unforced ILC, one sees a stronger periodicity in $\mathrm{x}$-direction due to forcing. Otherwise the phenomenology is very similar (movie available on enclosed CD-ROM).

In other experiments, described in the next sections, where transverse $\left(\varphi=90^{\circ}\right)$ and oblique $\left(\varphi=60^{\circ}\right)$ rolls are forced, crawling rolls were found as well which have a slightly different morphology.

\subsubsection{Patterns Driven by Shear Instability}

As can be seen in Fig. 2.2 for unforced ILC and inclination angles larger than $\gamma \approx 78^{\circ}$ instead of longitudinal rolls, transverse rolls with $\varphi=90^{\circ}$ become first unstable with increasing Rayleigh number. This is due to the large scale shear flow: Along the warm bottom plate fluid moves uphill while at the top plate cold fluid moves downhill. The shear stress at the interface between the warm and the cold fluid, causes this interface to become unstable. This is similar to the Kelvin-Helmholtz instability [52]. Therefore, even in inverse ILC, where the inclination angle is larger than $90^{\circ}$ and the warm fluid is above the cold one, rolls do occur with a specific wavelength [30].

For unforced ILC, there is no sharp transition at the codimension-two point $\left(\gamma_{c d}=\right.$ $78^{\circ}$ ) at which for smaller inclination longitudinal rolls and for larger inclination transverse rolls set in. Instead two transition states occur. At angles, slightly smaller than $78^{\circ}$ transverse bursts (TB) occur, while for slightly bigger angles a bimodal (BM) structure is selected [48].

In forced ILC one would expect that due to forcing, longitudinal rolls are stabilized and $\gamma_{c d}$ is shifted to higher inclination angles. Indeed, one observes patterns, very similar to TB and BM of unforced ILC. Surprisingly the range of $\gamma$ where transverse bursts exist, is strongly increased and the boundaries are shifted to both sides of $\gamma_{c d}$ (see Fig. 4.3). 

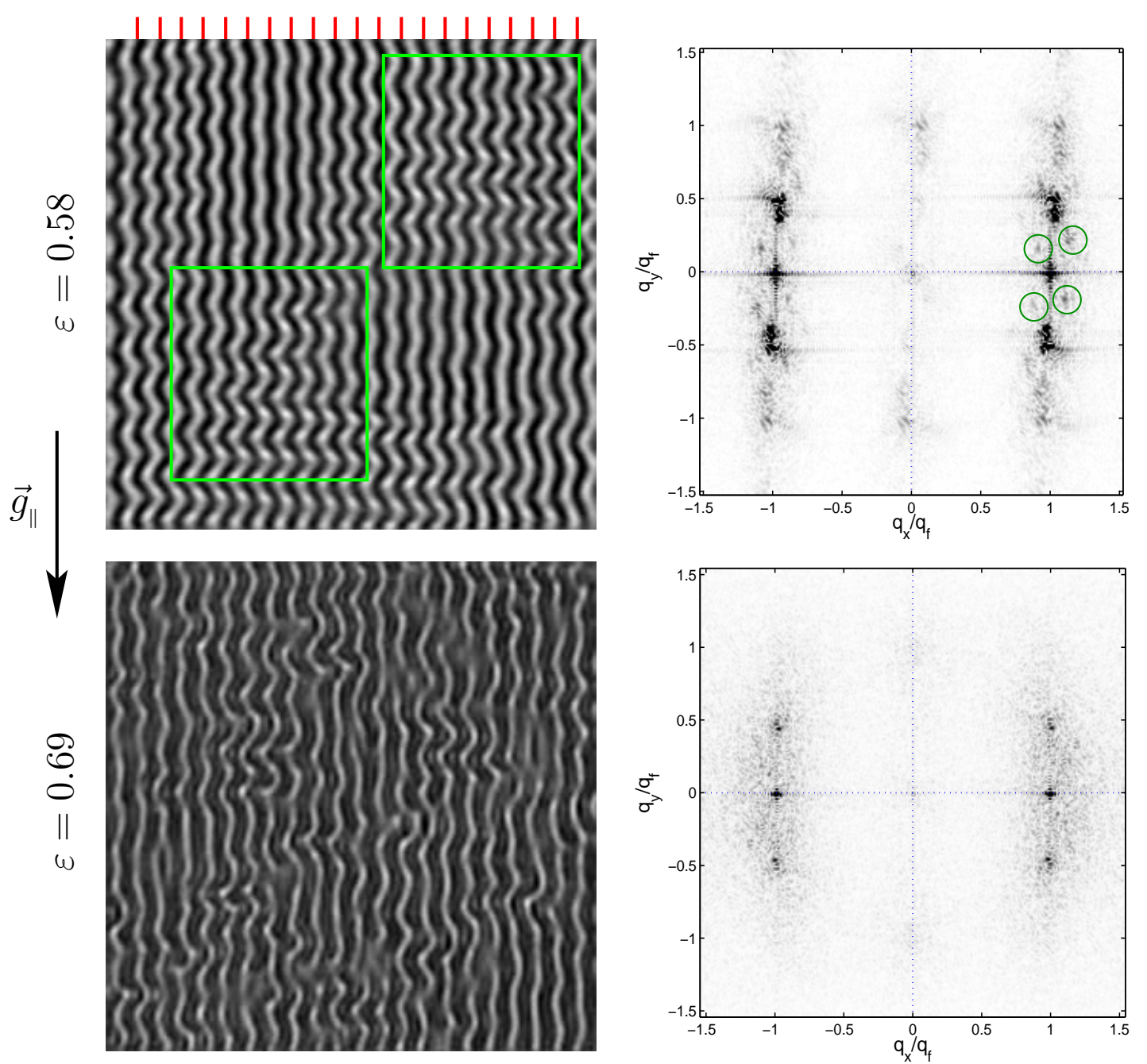

Figure 4.11: Undulation chaos (upper row) and crawling rolls (bottom row). Convection pattern (left) and the corresponding Fourier transform (right) for $\gamma=45^{\circ}$ and various $\varepsilon$. Note that the pattern are sections of size $43 d$ while the Fourier transform was done over the whole cell. Green rectangles mark areas with strong phase modulations. Green circles mark the appearance of small side modes next to the undulation modes (green circles). The red stripes mark the position of the bottom plate texture. 
Transverse bursts (TB) are observed in forced ILC for $75^{\circ} \leq \gamma \leq 82^{\circ}$. These bursts are dynamical 2-dimensional structures and occur spontaneously as a periodic modulation of longitudinal rolls in the transverse direction as shown in Fig. 4.12 (movie available on enclosed CD-ROM). The modulation grows quickly, and the burst pattern reaches its largest extend after $\approx 0.35 t_{\kappa}$. From then, the flow structure becomes unstable and looses its periodical order. It seems as if the periodic structure is flushed away by the large scale shear flow.

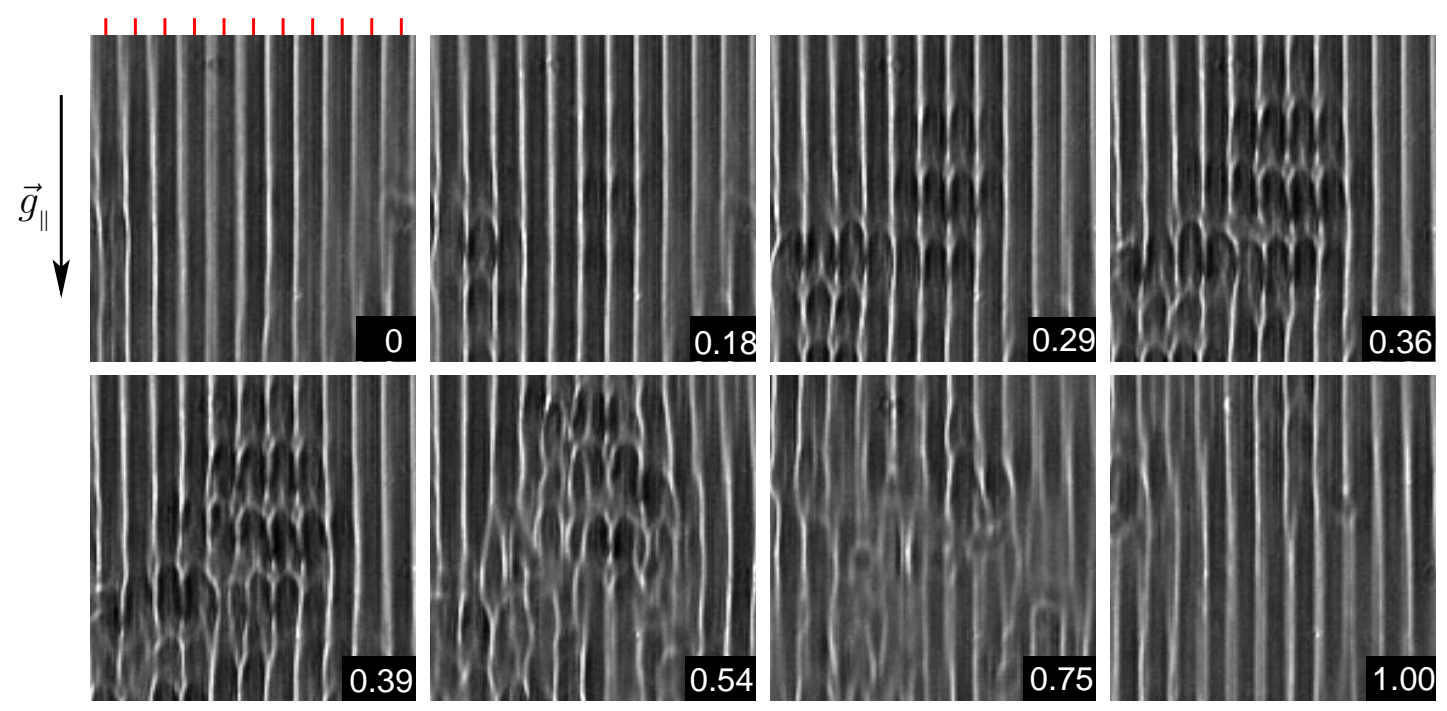

Figure 4.12: Time evolution of transverse bursts (TB). Images are sections of $21 d$ taken at $\gamma=80^{\circ}$ and $\varepsilon=0.17$. Numbers in the bottom left corners of images are times in units of vertical heat diffusion time $\left(t_{\kappa}\right)$. The small red lines in the first image mark the location of the forcing ridges.

These localized bursts do appear in small arrays of three to six bursts in the transverse direction and two to three in the longitudinal direction. The periodicity in longitudinal direction is twice as long as the forcing wave length (wave vector $\left.\vec{q}_{1}=(0,0.5) q_{f}\right)$. It is important to note that the periodicity in the transverse direction is the forcing wave length (Fig. 4.13) (wave vector $\left.\vec{q}_{f}=\left(q_{f}, 0\right)\right)^{8}$

Figure 4.14 shows the standard deviation of the shadowgraph image, the amplitude of the Fourier mode $\vec{q}_{1}$, and the amplitude of the forcing mode $\vec{q}_{f}$ as a function of time for the burst structure shown in Fig. 4.12. At $t=0$ a longitudinal roll pattern is present so that the amplitude of $\vec{q}_{1}$ is nearly zero, while the amplitude of $\vec{q}_{f}$ has already a finite value. When a burst array occurs the amplitudes of both modes increases. The forced mode reaches first a maximum

\footnotetext{
${ }^{8}$ The longitudinal direction is parallel to gravity, while the transverse direction is perpendicular to it. Calling a mode transverse means that the corresponding rolls are transverse, but the wave vector shows in longitudinal direction.
} 

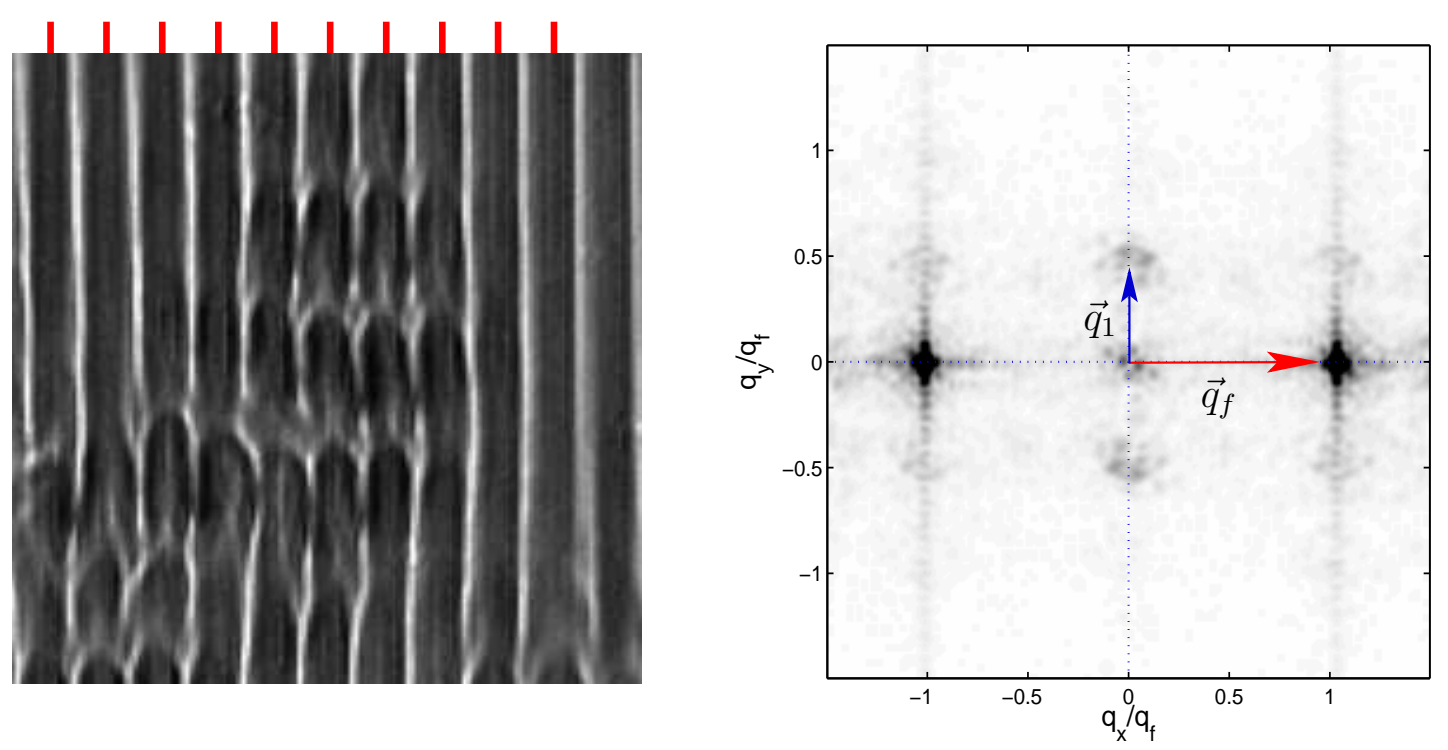

Figure 4.13: Array of transverse bursts and its Fourier transform at $\gamma=80^{\circ}$ and $\varepsilon=0.17$. The red stripes mark the forcing texture.

value after about $0.17 t_{\kappa}$ and starts to decrease from this point on. The amplitude of $\vec{q}_{1}$ continues to increase and reaches a maximum at $t=0.36 t_{\kappa}$ before it decays again. From this point on, the whole pattern becomes unstable and in the shadowgraph image it looks as if the pattern is flushed away (compare Fig. 4.12). It is interesting that the longitudinal mode does almost disappear at $t=0.7 t_{\kappa}$, whereas the mode $\vec{q}_{1}$ has still a small but finite amplitude. The standard deviation of the image in real space reaches its minimum later at $t \approx 0.81 t_{\kappa}$. From that point on, the amplitude of $\vec{q}_{f}$ starts to increase again while the amplitude of $\vec{q}_{1}$ has almost disappear. After $t \approx 1.1 t_{\kappa}$ the burst array has disappeard and longitudinal rolls are reinstalled. If one looks very carefully, one sees, that the amplitude of $\vec{q}_{1}$ starts to increase again for $t>1.1 t_{\kappa}$ since the next burst is going to appear.

The plots reveal a strong coupling of the longitudinal mode and the side mode $\vec{q}_{1}$. A strong mode in longitudinal direction $\left(\vec{q}_{f}\right)$ excites the transverse mode $\left(\vec{q}_{1}\right)$, while a strong transverse mode suppresses the longitudinal mode. These mechanisms lead to a reduced stability of longitudinal rolls. As can be seen in the Fig. 4.3, the onset of TB appears for even lower $\varepsilon$ as for transverse rolls (TR) at higher inclination angles.

A detailed description of TB in unforced ILC is given in [53]. TB in forced and unforced ILC are very similar in their phenomenology and in their short term dynamics. Long time measurements and long term statistics as presented in [53] were not done for the forced case. The difference between the forced and the unforced ILC is only expressed in the range of inclination angles at which TB 


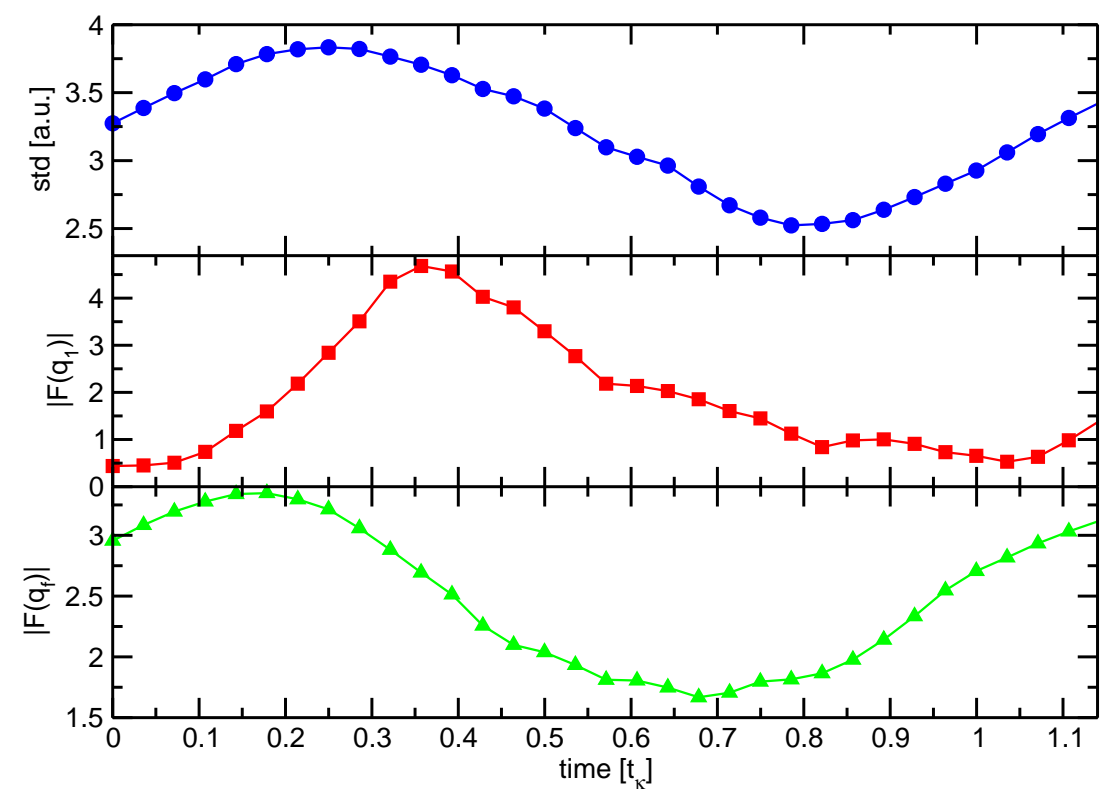

Figure 4.14: Standard deviation of the gray value of the burst array shown in Fig. $4.12(\bullet)$, the Fourier amplitude of the side mode $\left|F\left(\vec{q}_{1}\right)\right|(\square)$ and the Fourier amplitude of the forced mode $\left|F\left(\vec{q}_{f}\right)\right|(\Delta)$ as a function of time. The units of the y-axis are arbitrary units.

were observed. While in the unforced case TB were only found in the direct vicinity of $\gamma_{c d}\left( \pm 1^{\circ}\right)$, in forced ILC, TB were found for the range of $75^{\circ} \leq \gamma \leq 82^{\circ}$. This increase of the $\gamma$-range for which TB exist can be explained by the strong coupling between the longitudinal mode and the transverse mode $\vec{q}_{1}$.

For even larger inclinations, in the range of $82^{\circ} \leq \gamma \leq 90^{\circ}$, transverse rolls (TR) appear for small $\varepsilon$ and longitudinal bursts (LB) for higher $\varepsilon$. Transverse rolls were also observed in unforced ILC experiments [48] and could also be found in numerical simulations [51]. In the forced convection cell, the forced longitudinal rolls become first unstable to transverse rolls at $\varepsilon=0.05$.

These rolls are shown in Fig. 4.15 (movie available on enclosed CD-ROM). Here, even after background division, a strong modulation of TR in x-direction is still visible. This is not surprising, since numerical calculation [51] predicts a strong coupling between the transverse and the longitudinal mode. In unforced ILC a transverse mode is always coupled with a longitudinal mode even though for higher $\varepsilon$ and $\gamma$ the amplitude of the transverse component is dominant over the 
longitudinal one. In our experiments the longitudinal amplitude is even more enhanced by the longitudinal forcing.
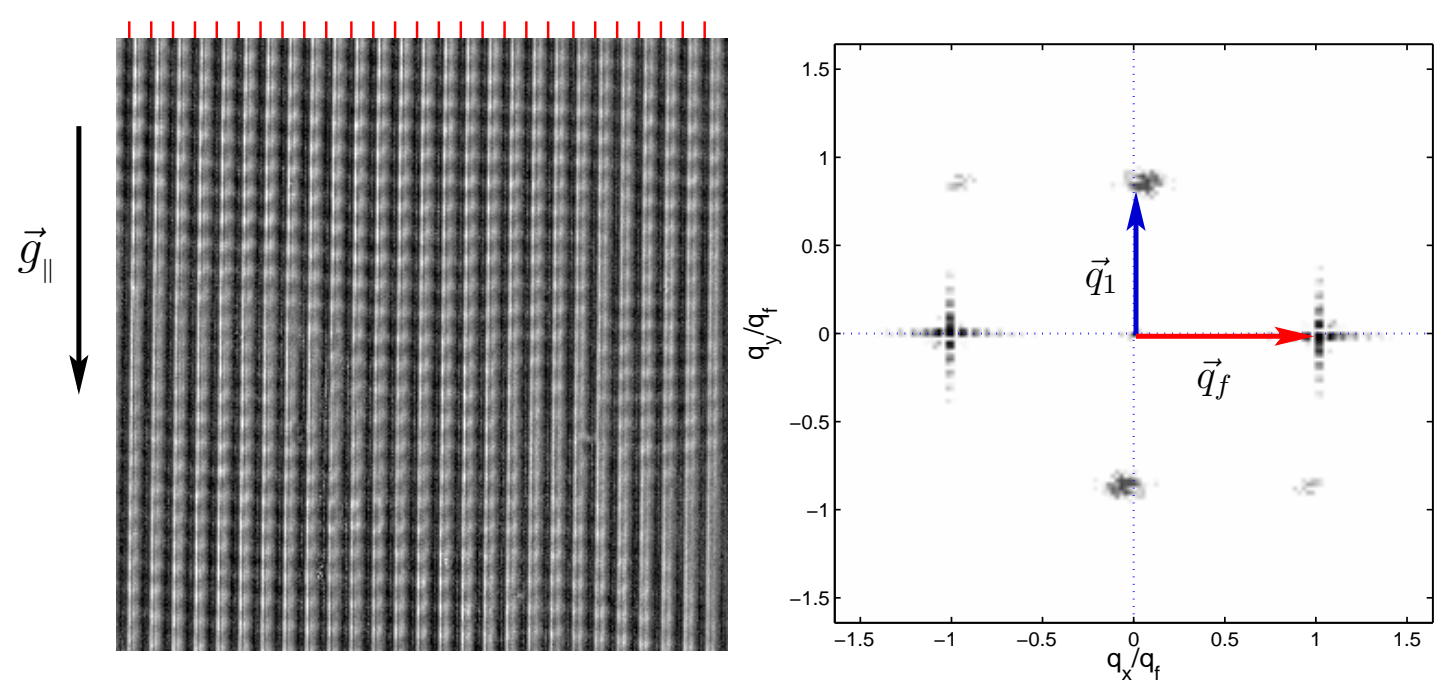

Figure 4.15: Transverse rolls and their corresponding Fourier transform at $\gamma=0^{\circ}$ and $\varepsilon=0.1$. The right image shows a Fourier-filtered version of the left image after removing the forced mode $\vec{q}_{f}$. The red stripes on top of the left image mark the forcing structure at the bottom plate.

Figure 4.15 shows the Fourier modes $\vec{q}_{f}$ and $\vec{q}_{1}$ for the transverse rolls. The second mode has a modulus of $\left|\vec{q}_{1}\right| \approx 0.84 q_{f}=2.86 / d$ which is close to the theoretically predicted value of the unforced ILC of $q_{\text {theory }} \approx \frac{2.82}{d}$ (blue curve in Fig. 2.3). The difference may be explained by a reduced shear flow in the forced cell due to is lower averaged cell height. An in this way reduced shear flow is somehow equivalent to a smaller inclination angle and thus, the occurring transverse rolls have a smaller wavelength (see Fig. 2.3).

Also here, the second mode $\vec{q}_{1}$ has also a small component in x-direction (Fourier transform in Fig. 4.15). The two peaks are expected to be symmetric with respect to the $\mathrm{x}$-axis, since in the unforced case TR occur as a linear instability at convection and is supposed not to couple with onset and but are in fact slightly inclined by an angle of $\approx 4^{\circ}$ against the y-axis. This is again due to a finite $\mathrm{x}$-component of the large scale shear flow as already described above for undulations. The same angle can also be seen after Fourier filtering, by which the forced mode $\vec{q}_{f}$ is removed as shown in Fig. 4.16.

The transversal roll pattern drifts in downhill direction with a velocity of $v_{d r i f t} \approx$ $2 d / \tau_{\kappa}\left(\right.$ for $\gamma=85^{\circ}$ and $\left.\varepsilon=0.1\right)$.

For higher $\varepsilon$ the transverse rolls become localy unstable to longitudinal bursts (LB) (Fig. 4.17). Here, a short roll segment of a length of $2 \lambda_{f}$ decouples from 
Figure 4.16: Fourier filtered transverse rolls after removing the forced mode $\vec{q}_{f}$. The image is the same as in Fig. 4.15.
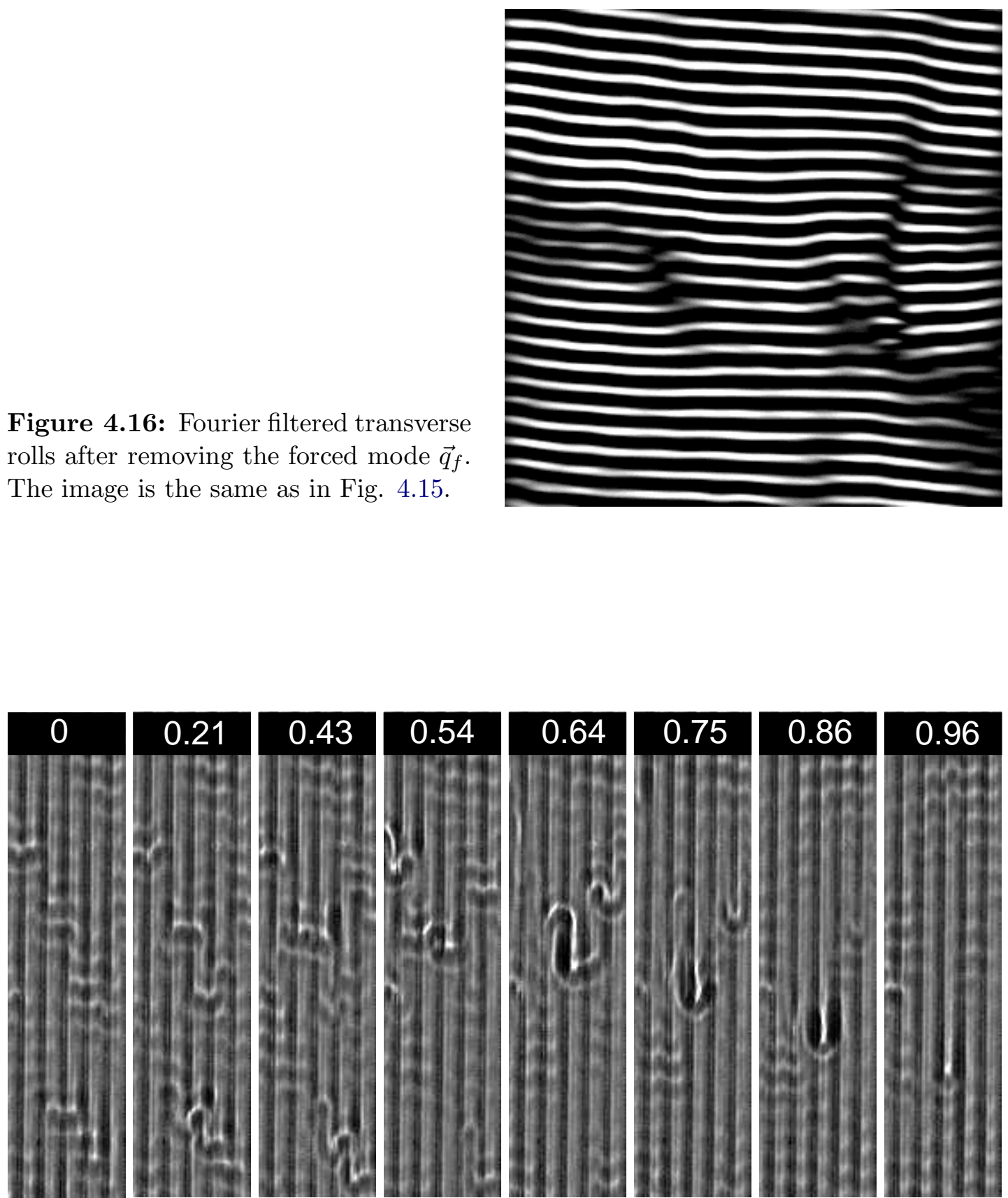

Figure 4.17: Time evolution of a longitudinal burst. The numbers are times in units of $\tau_{\kappa}$. 
the rest of the rolls and moves downward. Behind that roll an area appears, where short longitudinal roll segments appear for various times of a few $t_{\kappa}$. Figure 4.17 shows the time evolution of a burst. The typical relaxation time of a burst is of the order of 1-1.5 $t_{\kappa}$. Often, such a burst initiates also bursts in neighboring areas. These bursts usually occur when the amplitude of transverse rolls gets to large. Longitudinal bursts have been also observed in unforced ILC [48].

\subsubsection{Patterns at Low Inclination Angles}

The last subsection of this section, deals with patterns, that occur at very low inclination angles. These are often similar to patterns that exist in horizontal forced convection and that are discussed in detail in the next chapter. Several of these patterns coexist at the same time in different areas of the cell.

For small but finite inclination angles $\left(5^{\circ}<\gamma<10^{\circ}\right)$ varicose pattern (VP) coexist with coherent kink lines (KL). A very similar state were already found by McCoy and is described in detail in [69]. One kink is a localized phase jump of $2 \pi$ (Fig. 4.18a). These phase jumps are aligned on a line, which often spans over the whole cell. At onset of KL, each longitudinal roll has only one such phase kink. The kink line is mostly straight, but tend to bend towards the sidewalls. With increasing $\varepsilon$ more kink lines occur and are getting closer to each other. At sufficient high $\varepsilon$, kink lines cluster together and form patches of oblique rolls and of subharmonic resonances (Fig. 4.18b). Often varicose pattern form between adjacent kink lines (Fig. 4.18d). The kink lines drift slowly in downhill direction (movie available on enclosed CD-ROM) with a velocity of $\approx 0.7 d / \tau_{t}$ (for $\gamma=5^{\circ}$ and $\varepsilon=2.42$ ). The drift velocity increases with increasing shear flow (increase in $\varepsilon$ and $\gamma$ ).

The hysteretic behavior for varicose pattern and kink lines is different. In the horizontal case and at $\varepsilon$ at which VP exist, a reduction of $\varepsilon$ results in a reduced wiggling amplitude of VP in the same way as this amplitude grows with increasing $\varepsilon$. On the other hand reducing $\varepsilon$ from a spatio-temporal chaotic regime as in Fig. 4.8 or a kink state cannot reproduce VP anymore. Instead, straight longitudinal rolls form at some places of the convection cell, while in other regions kinks and kink clusters still exist (Fig. 4.18c). By reducing $\varepsilon$ the size of the kink cluster shrinks and finally disappears completely at much lower $\varepsilon$ (e.g., $\varepsilon=1.40$ at $\gamma=0^{\circ}$ ) as the instability of straight rolls occurred due to an increase of $\varepsilon$ $\left(\varepsilon=2.65\right.$ at $\left.\gamma=0^{\circ}\right)$.

At $\gamma=10^{\circ}$ one observes at $\varepsilon=2.16$ the onset of a varicose pattern. This varicose pattern is no longer steady in time, but drifts in uphill direction (movie available on enclosed CD-ROM). With increasing $\varepsilon$ one notices that the amplitude of the phase modulation is not constant over the whole cell anymore (see Fig. 4.19). In some areas the wickling - the amplitude of the phase modulation - is very strong 

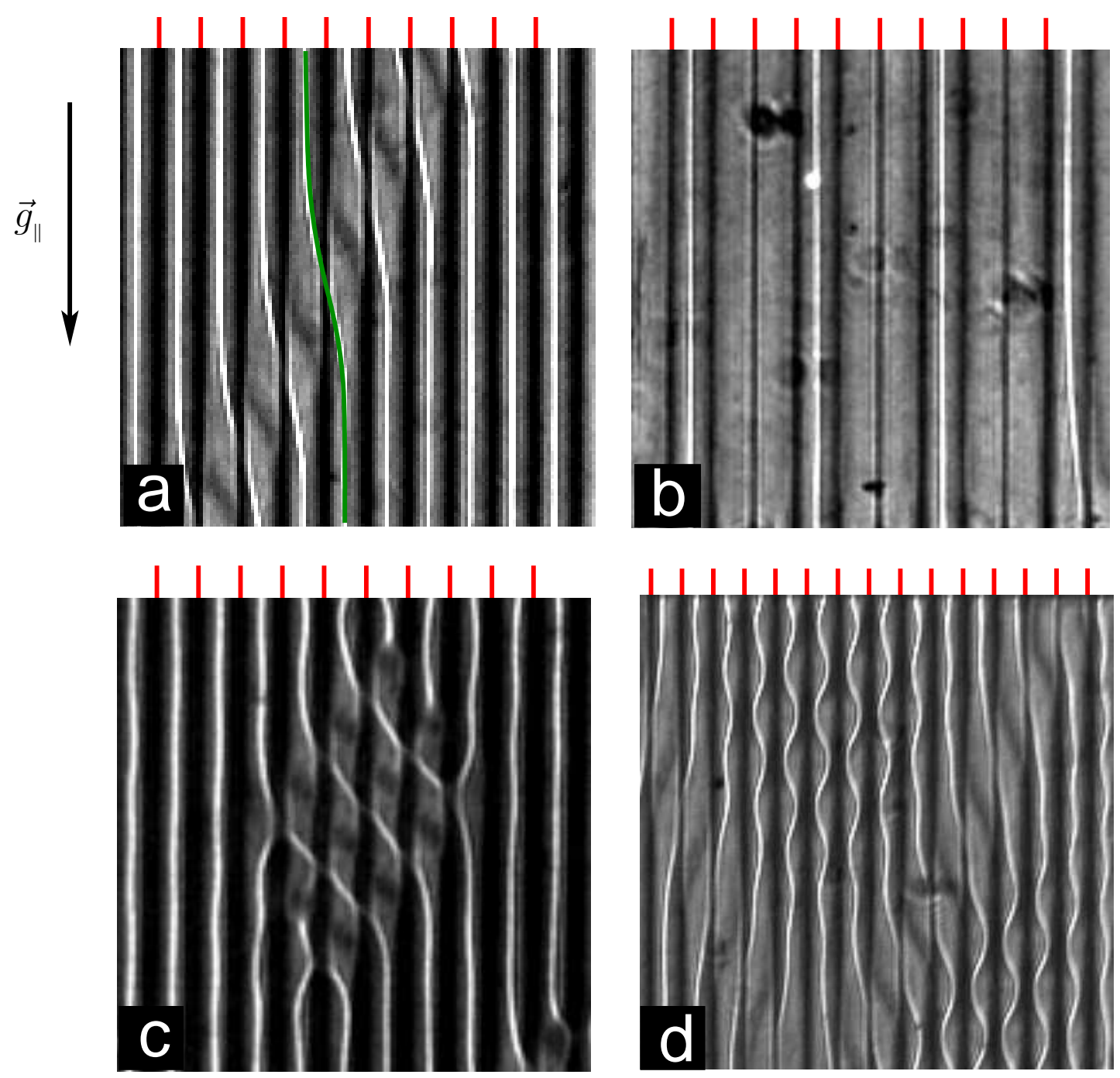

Figure 4.18: Single kink line at $\gamma=5^{\circ}$ and $\varepsilon=2.42$ (a). Subharmonic resonances at $\gamma=5^{\circ}$ and $\varepsilon=3.16$ (b). Kink cluster at $\gamma=0^{\circ}$ and $\varepsilon=1.69$ (c). Often, VP form in between adjacent kinks lines (d). The red stripes on top of each image mark the location of the forcing structure. 
while in other areas the rolls are almost straight.

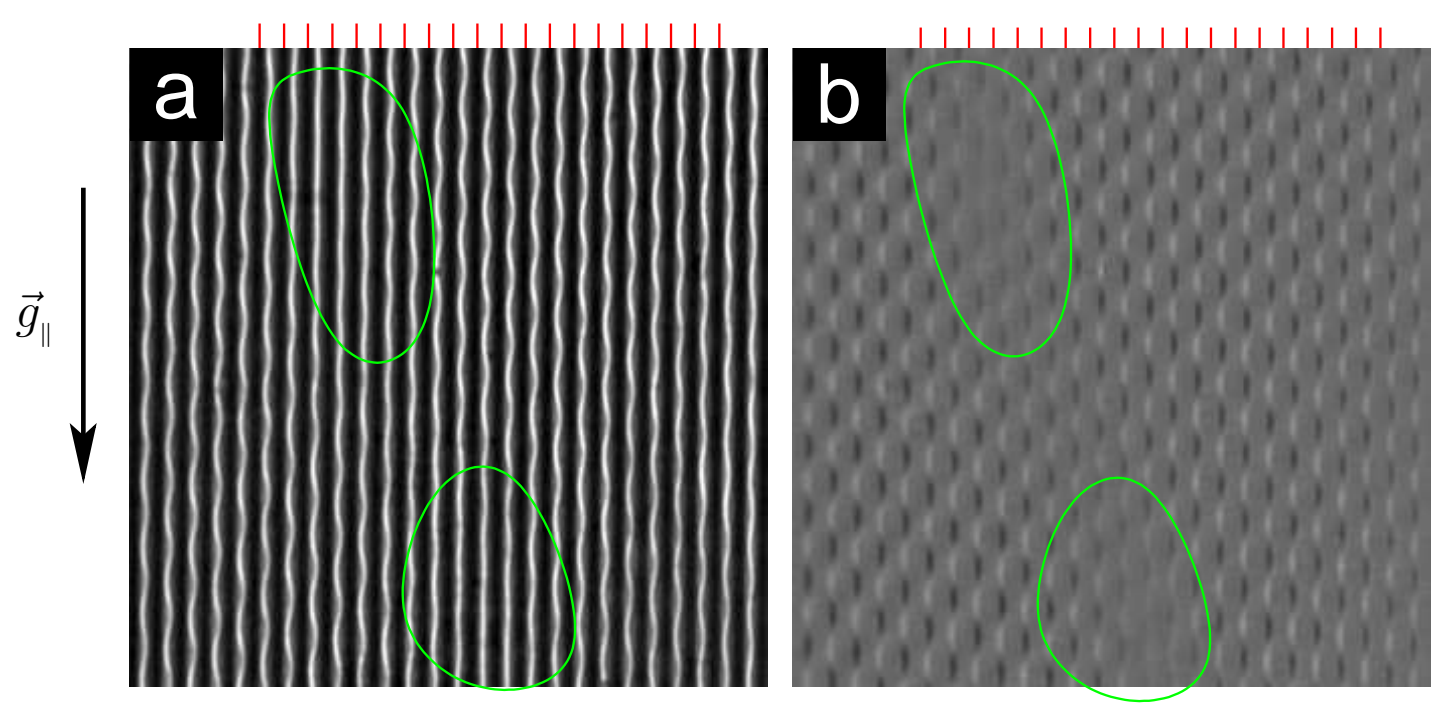

Figure 4.19: Varicose pattern at $\gamma=10^{\circ}$ and $\varepsilon=2.31$. (a) shows a shadowgraph image and (b) is the corresponding phase field. Green encircled areas mark areas, where the amplitude of the phase modulation is reduced. The red stripes on top of each image mark the location of the forcing texture.

At $\varepsilon=2.67$ neighboring rolls merge locally and produce kink defects (Fig. 4.20a). However, these kinks are neither very sharp steps in phase, nor are they aligned along a very straight line. Instead, they are only loose correlated in space and chaotic in time. This state looks like a mixture of varicose pattern, kink lines and crawling rolls. These crawling kinks (CK) are shown in Fig. 4.20b. They were observed only for inclination of $10^{\circ}$ and seem to be a mixed chaotic state, so that they are not marked in the phase diagram in Fig. 4.3.

Very interesting are these patterns if one lowers the control parameter and observes the evolution of CK. Here again a hysteretic behavior is observed. The pattern does not turn back to VP if one lowers $\varepsilon$. Instead, one observes small areas of size $\approx 3 \lambda_{f}$ in $\mathrm{y}$-direction and various length in $\mathrm{x}$-direction where a dynamic state exist (movie available on enclosed CD-ROM). There, dynamic crawling kinks with a subharmonic character were observed, while in the rest of the cell steady longitudinal rolls with wave vector $\vec{q}_{f}$ existed (see Fig. 4.21 and movie on enclosed CD-ROM). One even can observe these localized crawling kinks for $\varepsilon=1.67$. For comparison, in experiments were $\Delta T$ was adiabatically increased, forced longitudinal rolls were stable up to $\varepsilon=2.65$.

The fact that in a bistable regime a localized state is found is not unique to the system, presented here. Similar localized states were also found in other pattern forming systems. In binary convection localized traveling waves were 

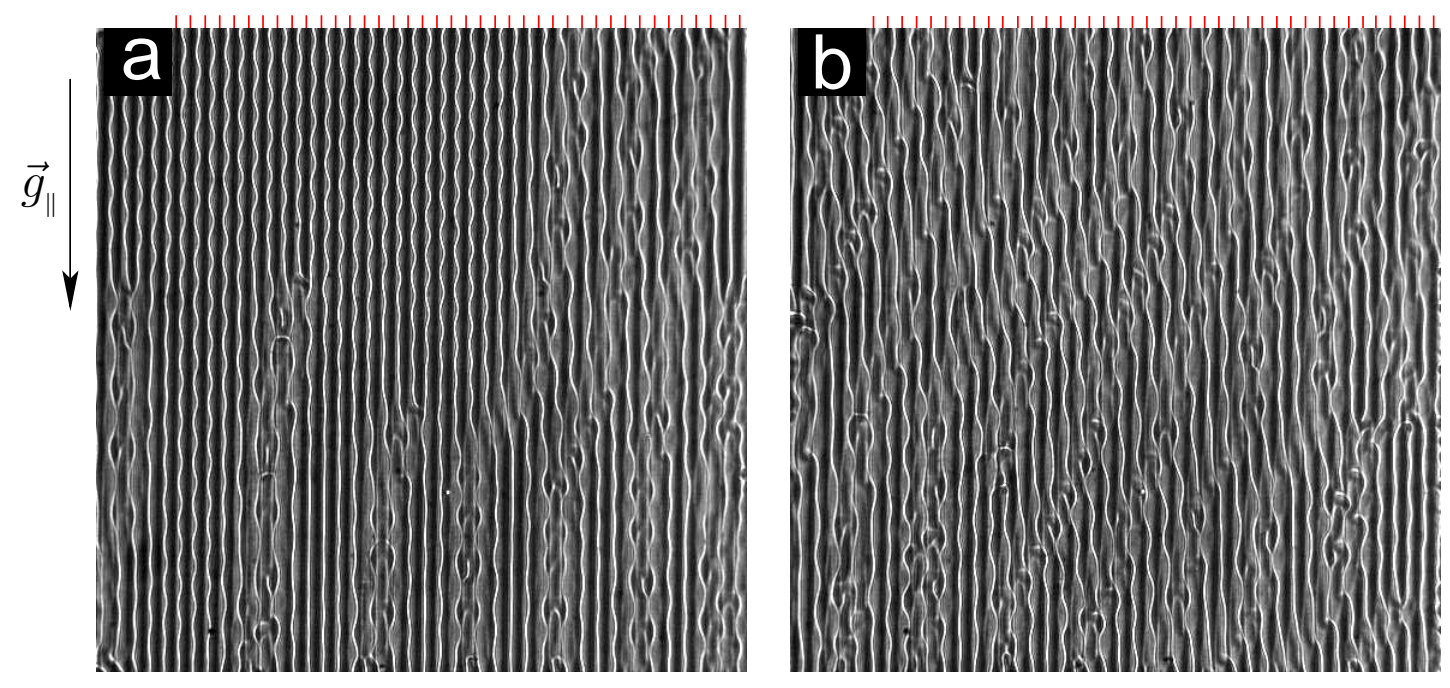

Figure 4.20: Crawling kinks and varicose pattern at $\varepsilon=2.67$ (a). Fully chaotic crawling kinks at $\varepsilon=2.90$ (b). Both images are taken at $\gamma=10^{\circ}$ and show the whole cell. The red stripes on top of the left image mark the forcing texture.

found embedded in on otherwise quiescent fluid, when the Rayleigh number was slightly higher then the critical one [89]. In Taylor Couette flow of dilute polymer solution for example localized vortex pairs where found when the rotation rate were reduced slowly from a state were chaotically oscillating vortices exists, to a simple Couette shear flow [90]. Another example are localized spikes in ferro fluids [90]. These occur when the fluid shows Rosensweig spikes and the magnetic field is slowly reduced. 


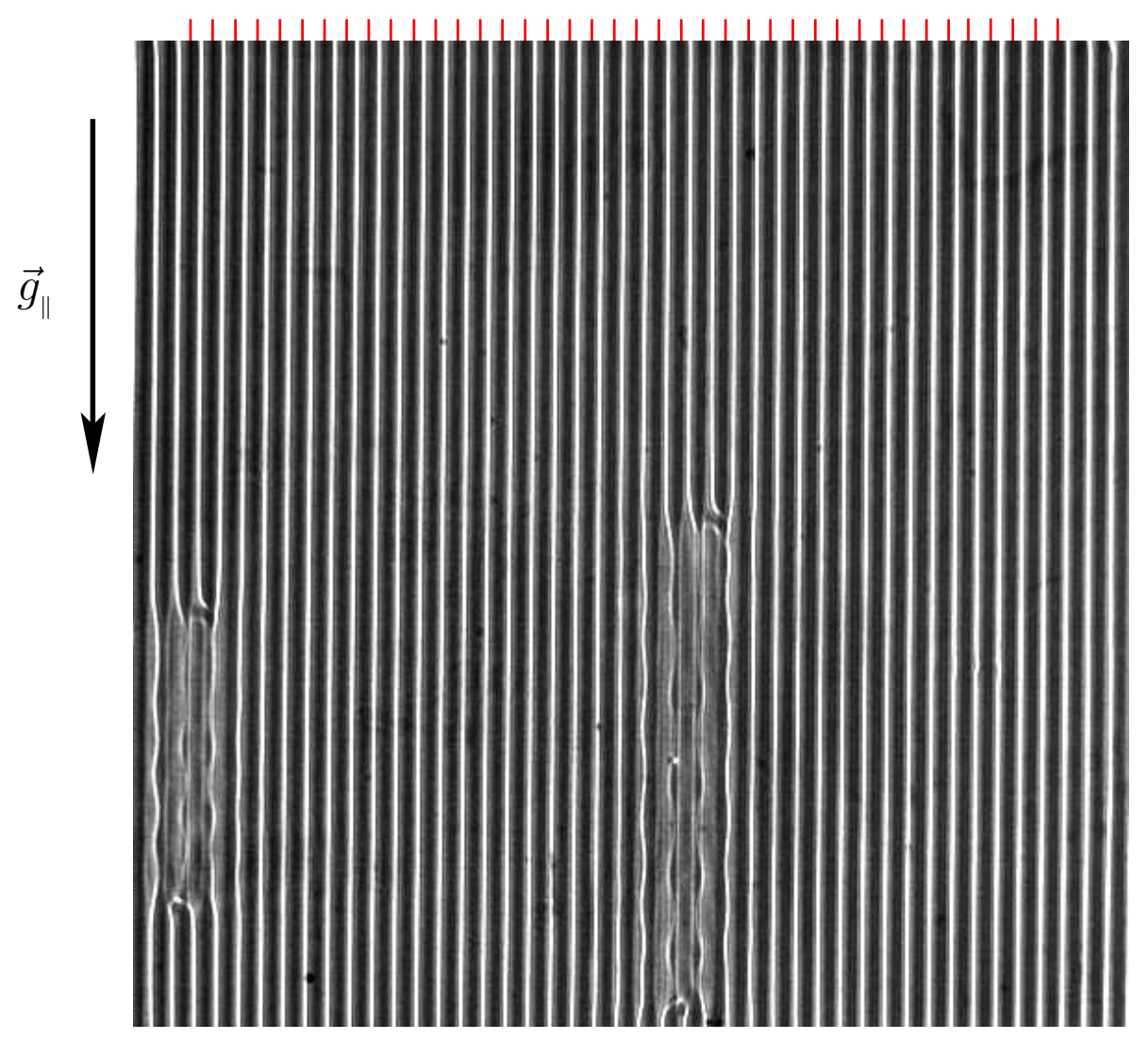

Figure 4.21: Localized crawling kinks at $\varepsilon=1.72$ and $\gamma=10^{\circ}$. The image show the whole cell. The red stripes mark the forcing texture.

\subsection{Forcing with a Wave Vector Parallel to Grav- ity - Transverse Forcing}

Now the case of transverse forcing is considered, where the angle between the in plane gravity component $\vec{g}_{\|}$and the forcing texture is $\varphi=90^{\circ}$. For small and moderate inclination angles $\left(\gamma<\gamma_{c d}\right)$, the two symmetry breaking mechanisms act in different directions. The large scale shear flow, due to inclination forces the convection rolls to be aligned with their axis parallel to gravity, whereas on the other hand, the bottom plate structure forces the convection rolls to be aligned perpendicular to gravity. By changing the inclination one can change the strength of the shear flow and tune in this way the relative strength of the two symmetry breaking mechanisms.

In this scenario, the large scale shear flow is periodically perturbed by the bumps at the bottom plate. While in the parallel forcing case most occurring patterns were similar to patterns found in unforced ILC, now new patterns occur due to 
the competition of both symmetry breaking mechanisms.

Figure 4.22 shows the phase diagram of the selected patterns in the $\gamma-\varepsilon$ parameter space. Patterns, such as rhombic pattern, hexarolls or bimodal pattern, occur due to instabilities of the forced transverse rolls. While these patterns are mostly steady in time, at higher $\varepsilon$ they turn into, dynamical states like crawling rolls or scepter pattern. Black lines in Fig. 4.22 are hand drawn guides to the eye marking the transitions between different states. The transition from forced transverse rolls to a new pattern is easy to recognize in the corresponding Fourier transform, while the transition to secondary instabilities, e.g., to crawling rolls, is driven by defect mediated instabilities resulting in a continuous increase of the dynamic and/or disorder and therefore difficult to recognize. Therefore, instead of plotting symbols for the transition between two patterns, symbols are plotted where a pattern is unambiguous.

Note, the logarithmic scaled $\varepsilon$-axis. That's why only patterns at positive $\varepsilon$-values are shown. For high inclination angles $(80<\theta<90)$, the forced transverse rolls become first unstable already at negative $\varepsilon$. For $\gamma=80^{\circ}$ bimodal pattern (BM) occur first at $\varepsilon \approx-0.18$ and turn at $\varepsilon \approx-0.09$ to heart pattern (HP). For larger inclination angles HP occur first as an instability of forced transverse rolls.

Transverse rolls, forced by the bottom plate structure have wave vector $\vec{q}_{f}=$ $\left(0, q_{f}\right)$. As shown in Fig. 2.2, these rolls are not preferred by the unforced inclined convection system and therefore, transverse rolls become, unstable at much lower $\varepsilon$ than the forced rolls in the longitudinal forcing case. In addition, up to $\gamma_{c d}$ where shear instability becomes important, the curve in the phase diagram (Fig. 4.22) that marks the instability of the transverse tolls has its minimum at $\gamma \approx 30^{\circ}$. This is close to the inclination angle at which the theoretically predicted onset curve for straight rolls with angle $\varphi=90^{\circ}$ has its maximum (dashed violet curve in Fig. 4.22). In sloppy words, at around $\gamma=30^{\circ}$ the system strongly "dislikes" transverse rolls.

\subsubsection{Rhombic Pattern}

For very small inclination angles $\left(\gamma \approx 5^{\circ}\right)$ the forced transverse rolls become unstable at $\varepsilon \approx 2.5$. This value is expected since all different forcing scenarios (all different $\varphi$ ) have to converge for very small inclination angles $\gamma \rightarrow 0$. The instability is induced by the boundaries and results in a pattern of longitudinal rolls. These rolls have defects and are not fully parallel to gravity but slightly oblique with various angles and directions (Fig. 4.23). In addition the roll amplitude is modulated periodically by the underlying forcing texture. At first glance it is not clear whether the obliqueness is produced by the interaction between forcing and inclination, or whether it is due to the influence of the lateral boundaries. Even in unforced RBC convection rolls tend to align perpendicular to lateral boundaries 


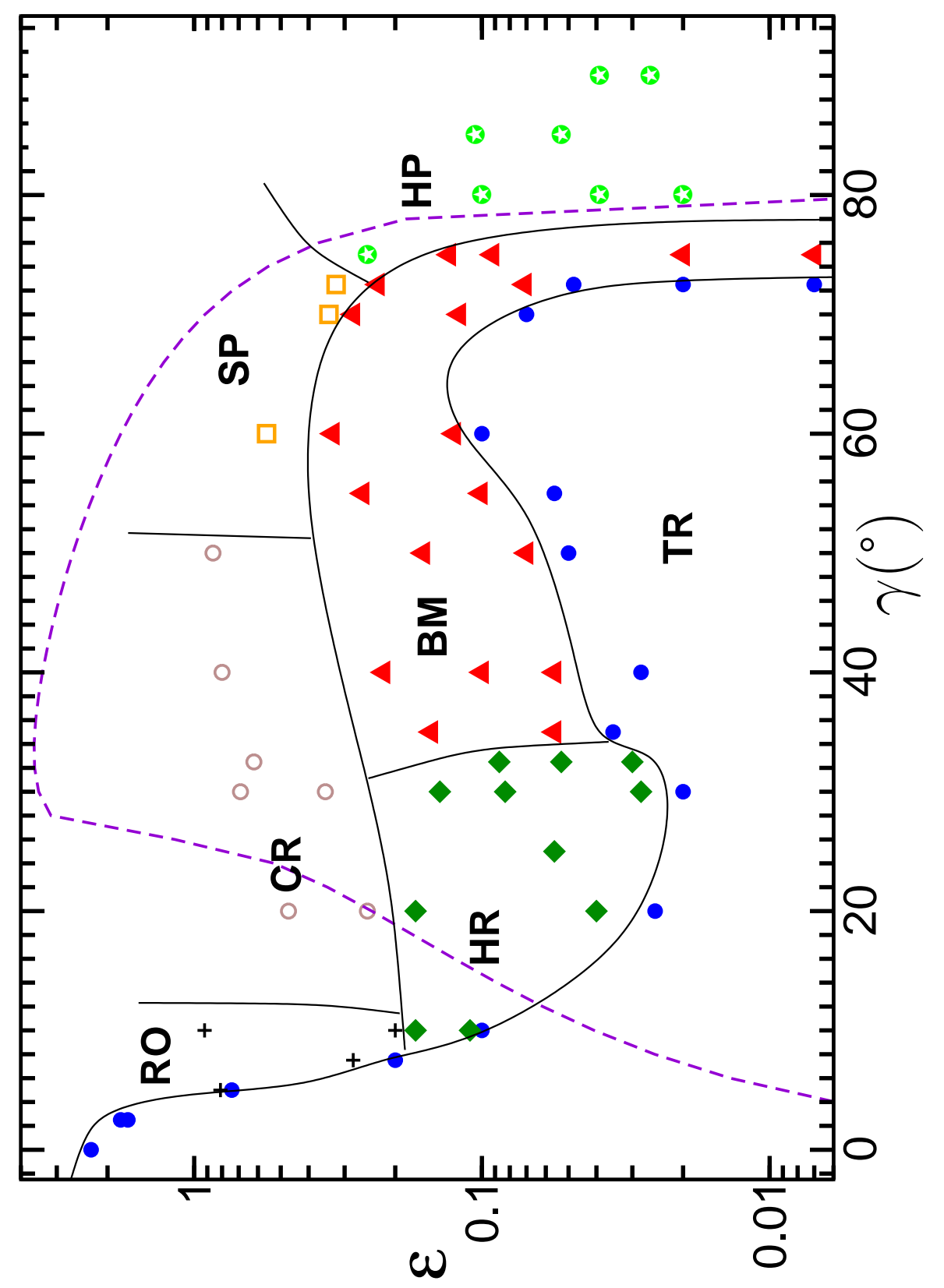

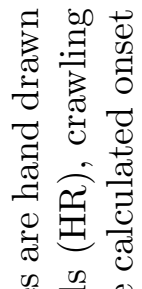

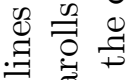

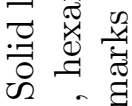

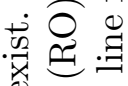

동

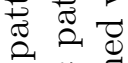

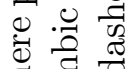

उ

कै च

के

若画

胥 을

a $0 \begin{aligned} & 0 \\ & 0 \\ & 0\end{aligned}$

है

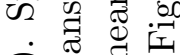

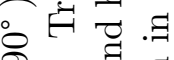

$\varnothing \ddot{\text { ส }}$

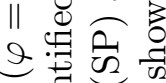

政

葡

跑

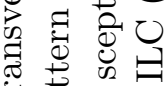

芯官

¿ी

द्व

.0.0

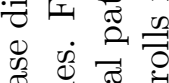

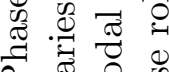

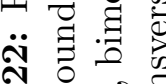

+0 에

을

垔 
and therefore produce curved and oblique rolls [30].

Figure 4.23: Longitudinal rolls at $\gamma=$ $5^{\circ}$ and $\varepsilon=0.81$. The red stripes mark the forcing structure.

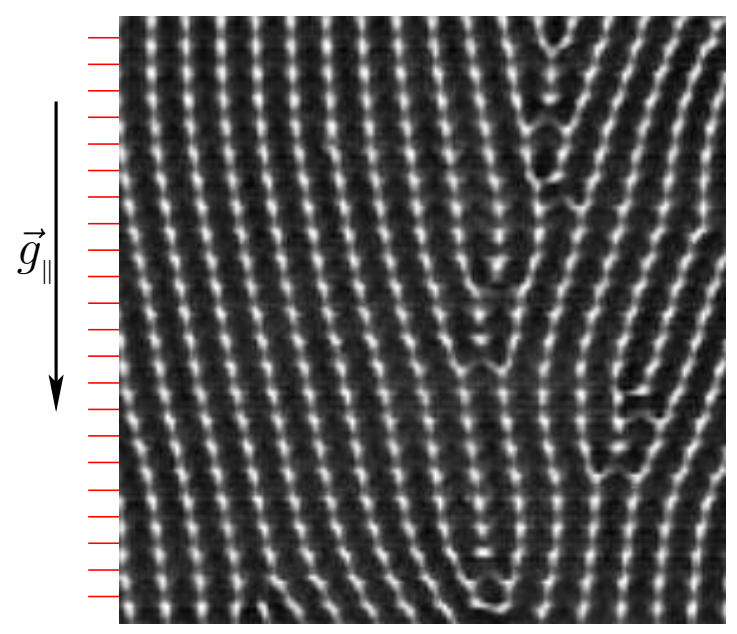

However, if one looks closer to the instability front, during the transition from transverse rolls to the new state, one sees that the instability starts with small periodic perturbations before the transverse rolls turn into longitudinal/oblique rolls 4.24. These modulations are varicose patterns, similar to VP as described in the previous section. Only the exact wave vectors differ slightly and are as can be seen in the Fourier transform (Fig. 4.25):

$$
\vec{q}_{1,2}=0.7 \cdot q_{f}\left(\begin{array}{c} 
\pm \sin \left(45^{\circ}\right) \\
\cos \left(45^{\circ}\right)
\end{array}\right)
$$

The instability results in an intermediate state where rolls are aligned $45^{\circ}$ to gravity and later on they turn to the described longitudinal/oblique roll state (at $500 \mathrm{~s}$ in Fig. 4.24).

A similar instability mechanism destabilizes the transverse rolls for $\gamma=7.5^{\circ}$. Although here, the destabilizing modes are not only visible at the transition itself but can also be seen in the resulting pattern - for sufficiently small $\varepsilon$. A typical example of the occurring pattern is shown in Fig. 4.26 together with its corresponding Fourier spectrum. This rhombic pattern (RO) is build by the forcing mode $\vec{q}_{f}$ and an additional mode:

$$
\vec{q}_{1}=s \cdot q_{f}\left(\begin{array}{c}
\sin (\alpha) \\
\cos (\alpha)
\end{array}\right)
$$

The length $s$ of $\vec{q}_{1}$ and the angle $\alpha$ change as one increases the control parameter, from $s=0.9, \alpha=30^{\circ}$ at $\varepsilon=0.25$ to $s=0.84, \alpha=10^{\circ}$ at $\varepsilon=0.5\left(\right.$ at $\gamma=10^{\circ}$ ).

The rhombic pattern does not occur as a global instability, but it does develop in patches as shown in Fig. 4.27. The patches grow in size with increasing $\varepsilon$. At a specific $\varepsilon$, areas with rhombic pattern coexist with areas of transverse rolls. These 

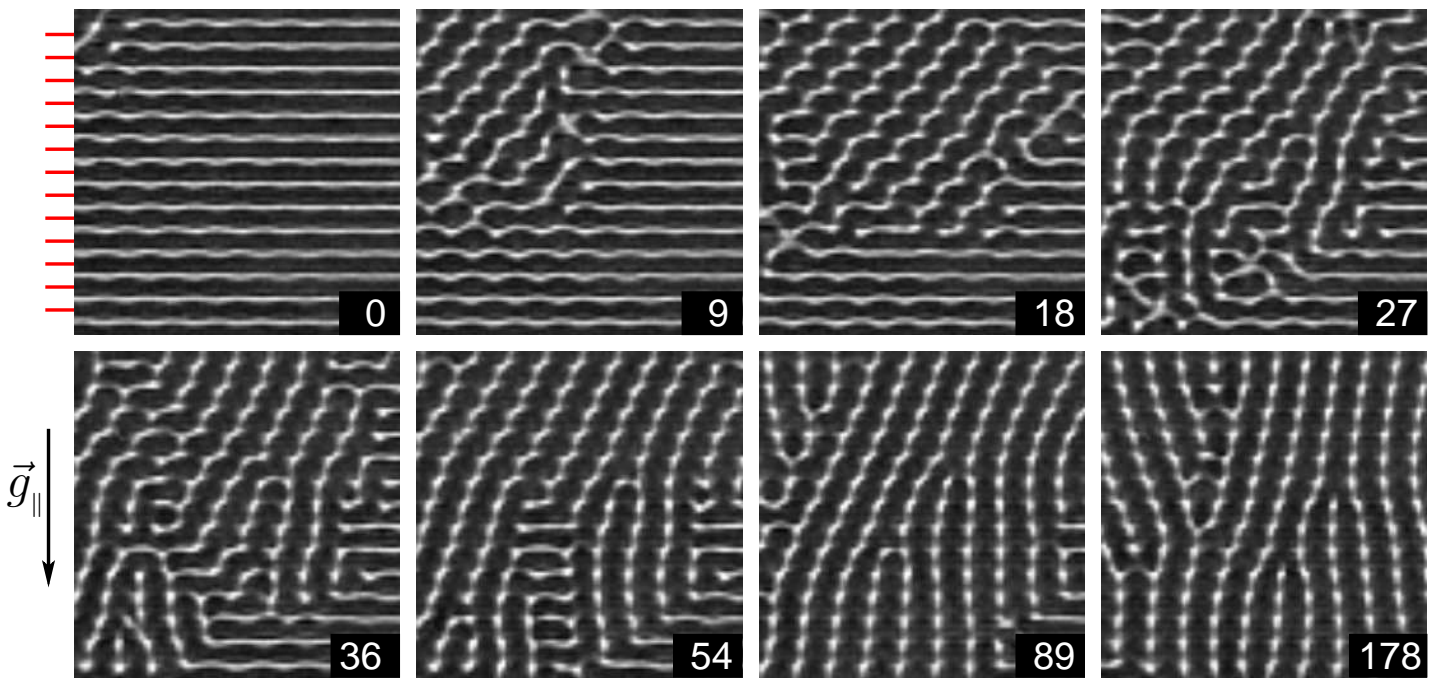

Figure 4.24: Evolution of rhombic pattern with time at very small inclination angles. Images taken at $\gamma=5^{\circ}$ and $\varepsilon=0.81$. The numbers in the corner of each images are the times in units of $\tau_{\kappa}$. The state after $178 \tau_{\kappa}$ is the final stable state of the system. The red horizontal stripes in the first image mark the locations of the forcing texture.

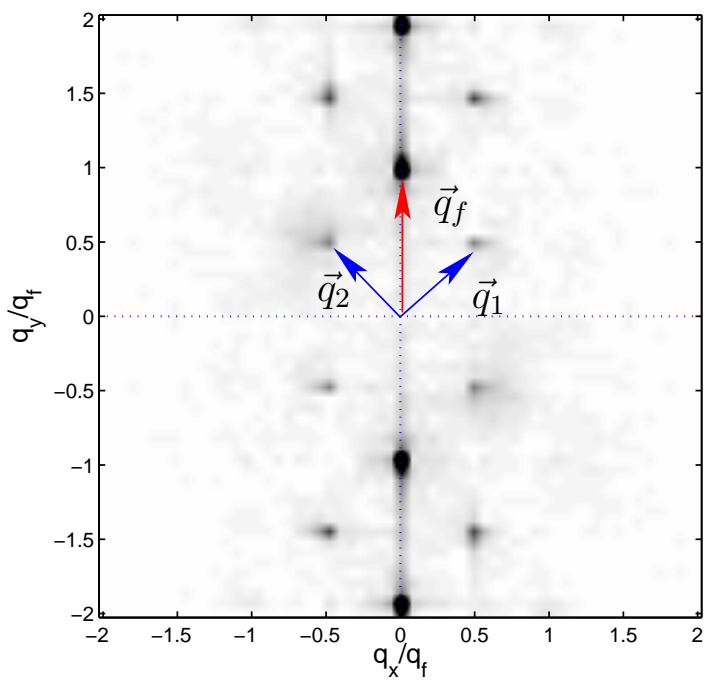

Figure 4.25: Fourier transform of varicose instability during evolution of rhombic pattern. The transform was taken of the first image in Fig. 4.24 

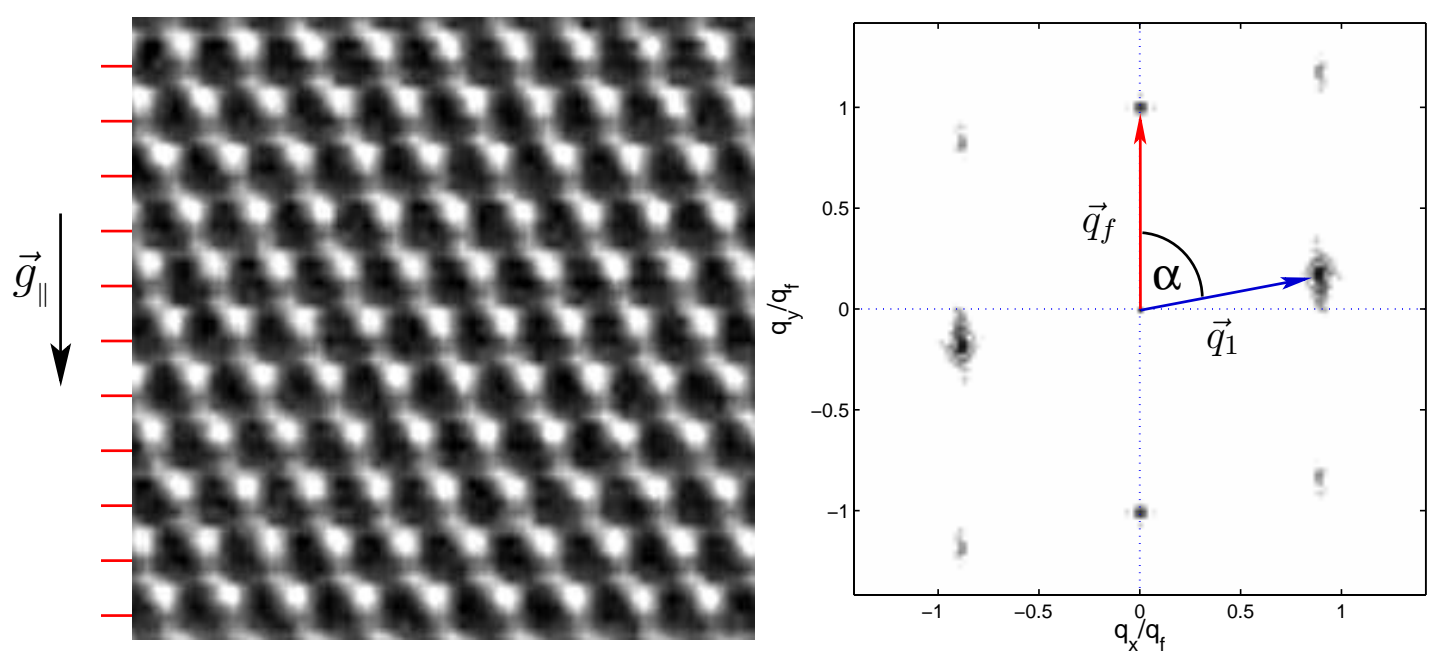

Figure 4.26: Typical rhombic pattern and its Fourier transform. Note that the Fourier transform was done over the whole cell and not only about the section shown in the image to the left. The red stripes mark the forcing texture.

areas fluctuate in time. While in some areas transverse rolls become unstable to rhombic pattern, in other areas rhombic pattern turn back to rolls.

The amplitude plots in Fig. 4.27 present the intensity of several modes in the Fourier spectrum, averaged over the whole cell. An increase of the amplitude of Fourier mode $\vec{q}_{1}$ includes an increase of areas with rhombic pattern. Green triangles represent the convection amplitude of the unforced reference cell, which follows the well known square root law. The amplitude of $\vec{q}_{f}$ is marked with ' + '. As long as transverse rolls are stable the forced amplitude follows a curve similar to the imperfect bifurcation curve shown in Fig. 4.2. At $\varepsilon \approx 0.24$ the instability to RO occurs first at some areas of the cell. Patches of straight rolls and rhombic pattern coexist all over the cell. Fluctuation takes place and while at some areas straight rolls turn into rhombic pattern at other places rhombic pattern turn into straight rolls. Averaged over the whole cell, the amplitude of $\vec{q}_{1}$ increases strongly at onset of RO but only slightly at $\varepsilon \approx 0.45$. At onset of RO, the amplitude of the forced mode $\vec{q}_{f}$ decreases significantly and stays constant for $\varepsilon>0.4$.

For larger $\varepsilon$ the large scale shear flow dominates over the forcing texture in the same way as for $\gamma=5^{\circ}$. This results in rhombic pattern longitudinal rolls that are modulated by the bottom plate texture but that are otherwise not different from rolls in unforced ILC. Such as state with defects and obliqueness due to side wall effects is shown in Fig. 4.28. 

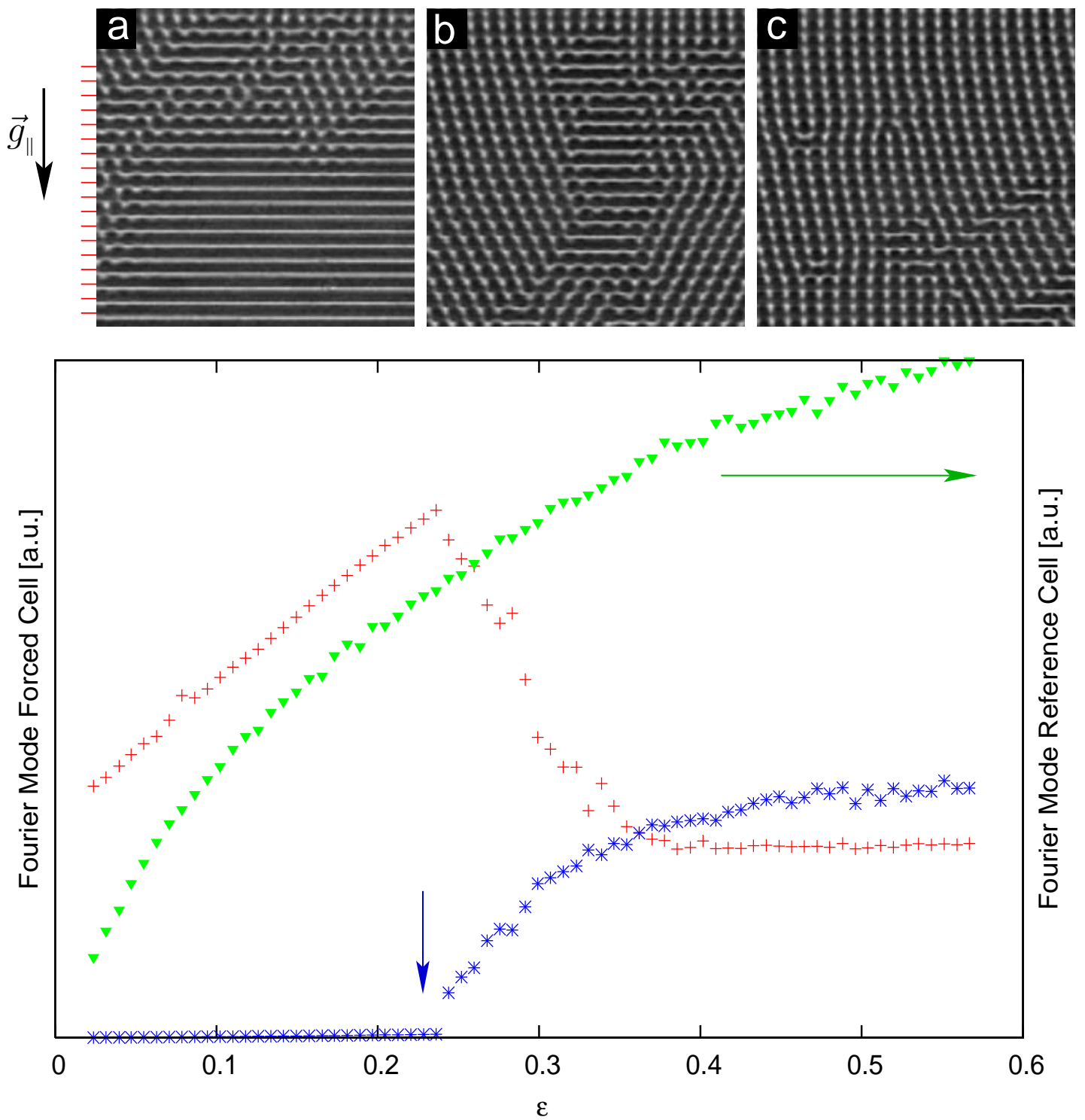

Figure 4.27: Evolution of rhombic pattern as a function of $\varepsilon$. Top: Shadowgraph images taken at $\gamma=7.5^{\circ}$ and $\varepsilon=0.25$ (a), $\varepsilon=0.28$ (b) and $\varepsilon=0.32$ (c). The red stripes on the left of (a) mark the forcing texture. Bottom: Development of the amplitudes of forced mode $\vec{q}_{f}(+)$ and instability mode $\vec{q}_{1}(*)$ in comparison to the convection amplitude in the reference cell $(\nabla)$. Experiments were done at $\gamma=7.5^{\circ}$. The blue arrow marks the instability onset. 


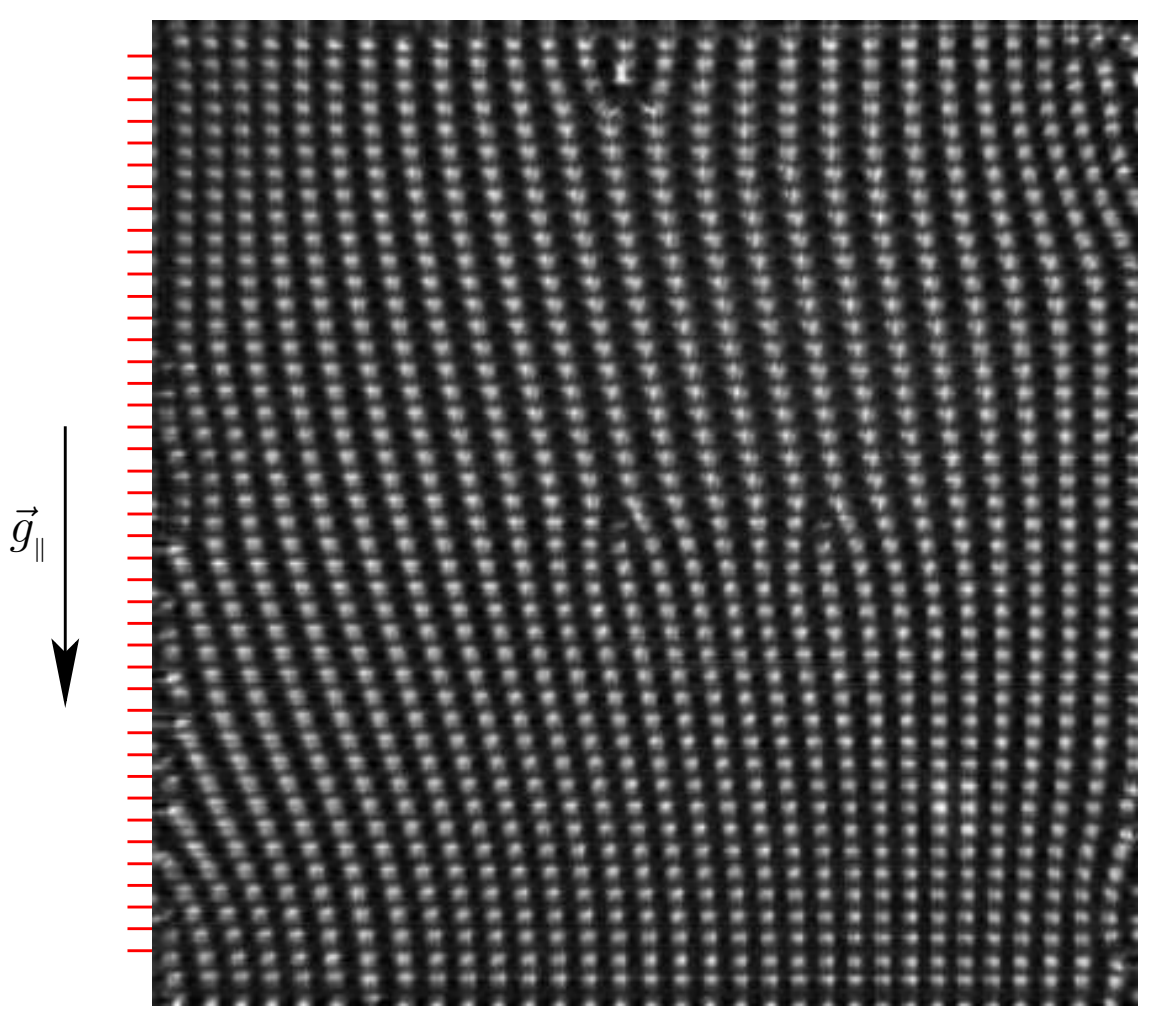

Figure 4.28: Rhombic pattern at $\gamma=5^{\circ}$ and $\varepsilon=3.7$. At that high $\varepsilon$, the large scale shear flow dominates over the forcing texture and aligns the pattern in longitudinal direction. The red stripes mark the location of the forcing structure.

\subsubsection{Hexarolls and Crawling Rolls}

At an inclination of $\gamma=10^{\circ} \mathrm{RO}$ only exist at around $\varepsilon=0.2$ and it is not the first occurring instability. Instead, already at $\varepsilon=0.11$ the forced transverse rolls with wave vector $\vec{q}_{f}=\left(0, q_{f}\right)$ become unstable with respect to two new modes $\vec{q}_{1}$ and $\overrightarrow{q_{2}}$ and a pattern occurs with a basic hexagonal symmetry as shown in Fig. 4.29. The observed patterns are not hexagons, but rolls with a hexagonal modulation. Similar hexaroll patterns (HR) were observed numerically [91, 51] and experimentally [92] in centrifugal convection.

For the whole range of inclination angles $10^{\circ} \leq \gamma \leq 20^{\circ}$ transverse rolls become unstable to hexarolls (left picture in Fig. 4.29). The additional modes as identified from the Fourier transform in Fig. 4.29 are:

$$
\begin{aligned}
& \vec{q}_{1} \approx 0.9 \cdot q_{f}\left(\begin{array}{c}
\sin \left(56^{\circ}\right) \\
-\cos \left(56^{\circ}\right)
\end{array}\right)=q_{f}\left(\begin{array}{c}
0.75 \\
-0.5
\end{array}\right) \\
& \vec{q}_{2} \approx 0.9 \cdot q_{f}\left(\begin{array}{c}
-\sin \left(56^{\circ}\right) \\
-\cos \left(56^{\circ}\right)
\end{array}\right)=q_{f}\left(\begin{array}{c}
-0.75 \\
-0.5
\end{array}\right) .
\end{aligned}
$$



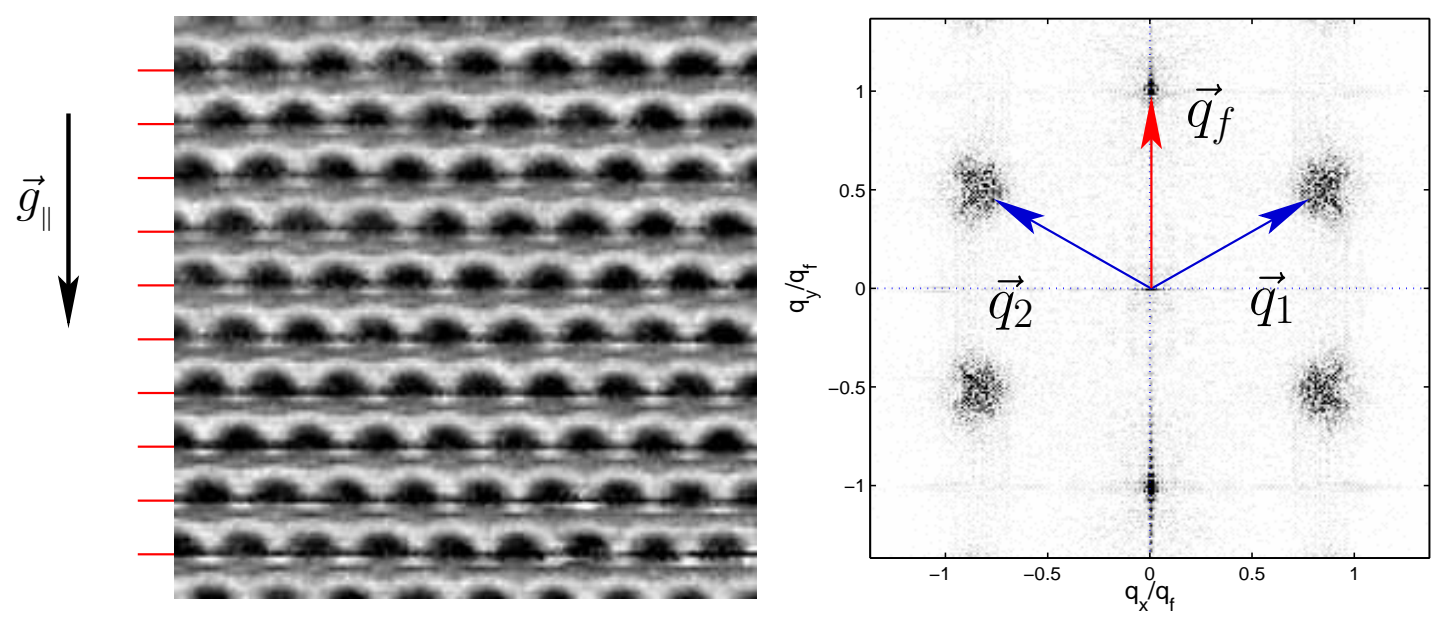

Figure 4.29: Hexarolls and its corresponding Fourier transform (taken at $\gamma=22.5^{\circ}$ and $\varepsilon=0.07$ ). The red stripes mark the location of the forcing structure.

Here again $\vec{q}_{1}$ and $\overrightarrow{q_{2}}$ are not always symmetric with respect to the y-axis but often show an inclination due to the lateral flow in horizontal direction that was mentioned already above. And again, all three wave vectors fulfill the triade resonant condition:

$$
\overrightarrow{q_{f}}+\overrightarrow{q_{1}}=\overrightarrow{q_{2}}
$$

A mathematical expression for hexaroll pattern can be given as a simple superposition of the three modes, whereas the amplitudes for modes $\vec{q}_{1}$ and $\vec{q}_{2}$ are equal and both are smaller than the amplitude of the forced mode $\left(A_{0}<A_{1}\right)$. In addition one has to take care about the right phases, therefore a $\sin ()$ occurs as third term:

$$
I_{H R}=A_{0} \cos \left(\vec{r} \vec{q}_{f}\right)+A_{1}\left(\sin \left(\vec{r} \vec{q}_{1}\right)-\cos \left(\vec{r} \vec{q}_{2}\right)\right) .
$$

Figure 4.30 shows a pattern, calculated with Eq. 4.9 and amplitudes $A_{0}=3$ and $A_{1}=1$. The difference to the experimental pattern in Fig. 4.29 is due to higher harmonics in the experiment that are not taken into account in Eq. 4.9.

In case of equal amplitudes $\left(A_{0}=A_{1}\right)$ one gets an array of triangles.

Hexaroll patterns are very stable in time and do not show any dynamic for sufficient small $\varepsilon$.

For higher $\varepsilon$, defects occur and start to destroy the hexagonal symmetry (Fig. 4.31). The modes in the Fourier spectra spread and start to move to the x-axis. The large scale shear flow is now stronger than the forcing effect of the bottom plate and aligns the rolls along the in-plane gravity component (longitudinal rolls). 
Figure 4.30: Calculated hexaroll pattern regarding Eq. 4.9. The amplitudes are: $A_{0}=3$ and $A_{1}=1$.

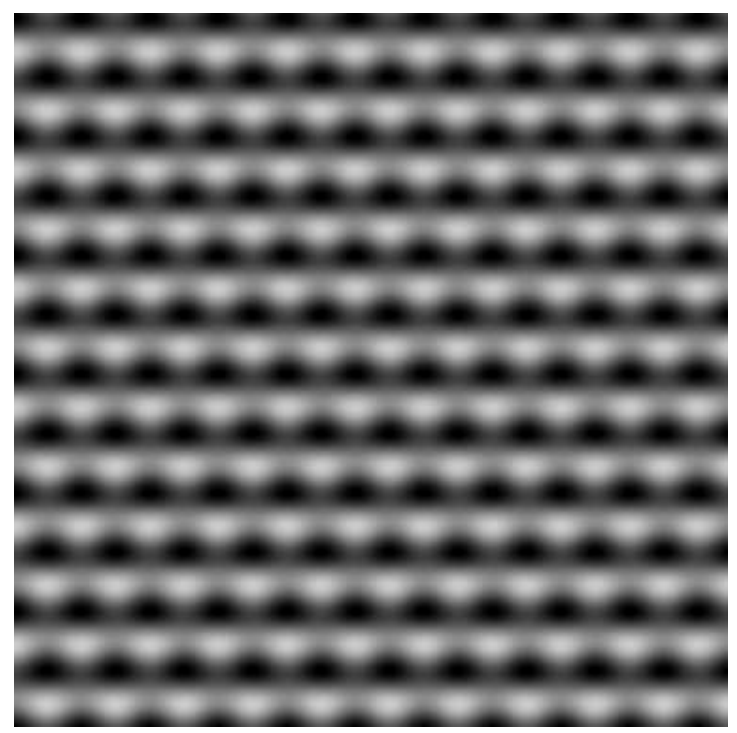

Since even in the unforced case, longitudinal rolls are no longer stable at that large $\varepsilon$, the system develops crawling rolls (CR) instead (movie available on enclosed CD-ROM). As mentioned in the previous section, the exact shape of CR is influenced by the forcing and therefore depends strongly on the alignment of the SU-8 texture. Close to the onset of CR the morphological differences to unforced $\mathrm{CR}$ are larger than for higher $\varepsilon$, since then the relative influence of the boundary texture is reduced.

Figure 4.31 shows the transition from a highly ordered hexaroll state to a strongly dynamical and defect turbulent crawling roll state for $\gamma=22.5^{\circ}$. In Fig. 4.31d, one sees the energy of the first quadrant of the power spectra $\left(|F(\vec{q})|^{2}\right)^{9}$ plotted as a function of the control parameter $\varepsilon$. The first two points are taken before the onset of hexarolls. Then, at $\varepsilon=0.01$, two new modes occur and build up the hexagonal pattern. While the intensity of the forced mode $\left(\vec{q}_{f}\right)$ stays constant, the intensity of the new mode $\vec{q}_{1}$ grows following a pitchfork-like bifurcation. Note, that the intensity in the power spectra is plotted in Fig. 4.31d which is the square of the Fourier mode. Thus, the linear increase of intensity in the power spectra corresponds to a square root increase of the intensity in Fourier space. Arrows are drawn in the left plot of Fig. 4.31 corresponding the $\varepsilon$ at which the images of above were taken. At $\varepsilon \approx 0.17$ the transition from hexarolls to crawling rolls takes place. This results in a stronger increase of intensity in the first FFT quadrant.

Beside the amplitude, the orientation and the length of the vectors $\vec{q}_{1}$ and $\vec{q}_{2}$ change as well in the way shown in the right plot of Fig. 4.31e. During the evolution of hexarolls the length of wave vectors $\vec{q}_{1}$ and $\overrightarrow{q_{2}}$ increase monotonically

\footnotetext{
${ }^{9}$ The first quadrant are all the points with $q_{y}>0$ and $q_{x}>0$. For measurement an area was chosen, so that the forcing peak is not included.
} 
(a)

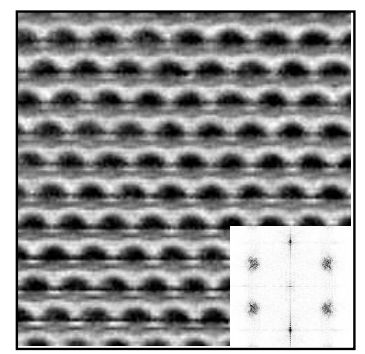

(b)

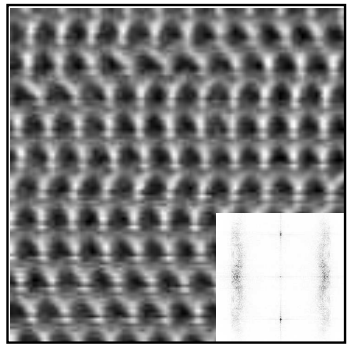

(c)

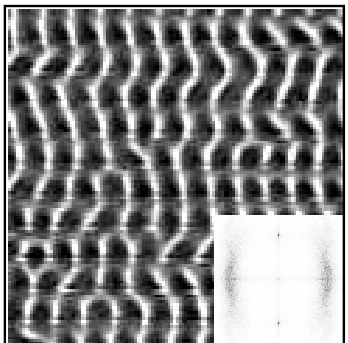

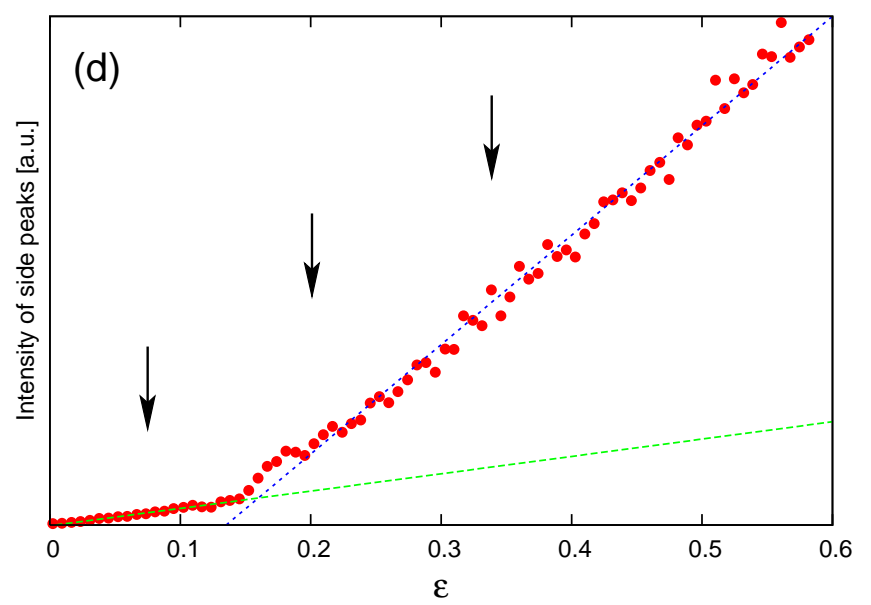

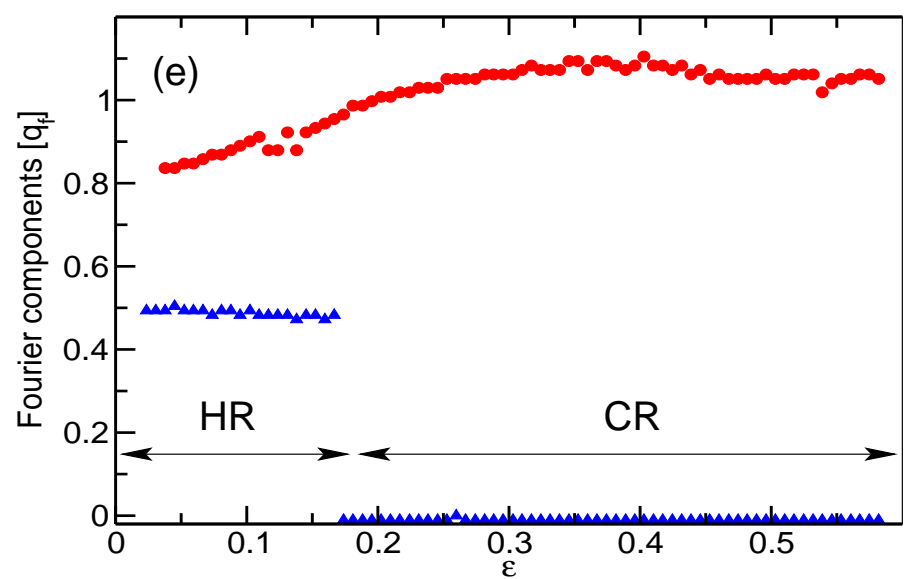

Figure 4.31: Hexarolls and the transition to crawling rolls for $\gamma=22.5^{\circ}$. Pictures show typical pattern at different $\varepsilon$ and their corresponding Fourier transform. The plot (d) shows the integrated intensity of the power spectra over the 1st quadrant as a function of the control parameter $\varepsilon$. Arrows mark the data points for the pictures above. Lines were fitted as a guide for the eyes. Plot (e) shows the length $\left|\vec{q}_{1,2}\right|(\bullet)$ and the y-component $\hat{y} \vec{q}_{1,2}(\mathbf{\Lambda})$ of the position of the maximum in the Fourier transform for $\gamma=22.5^{\circ}$. 

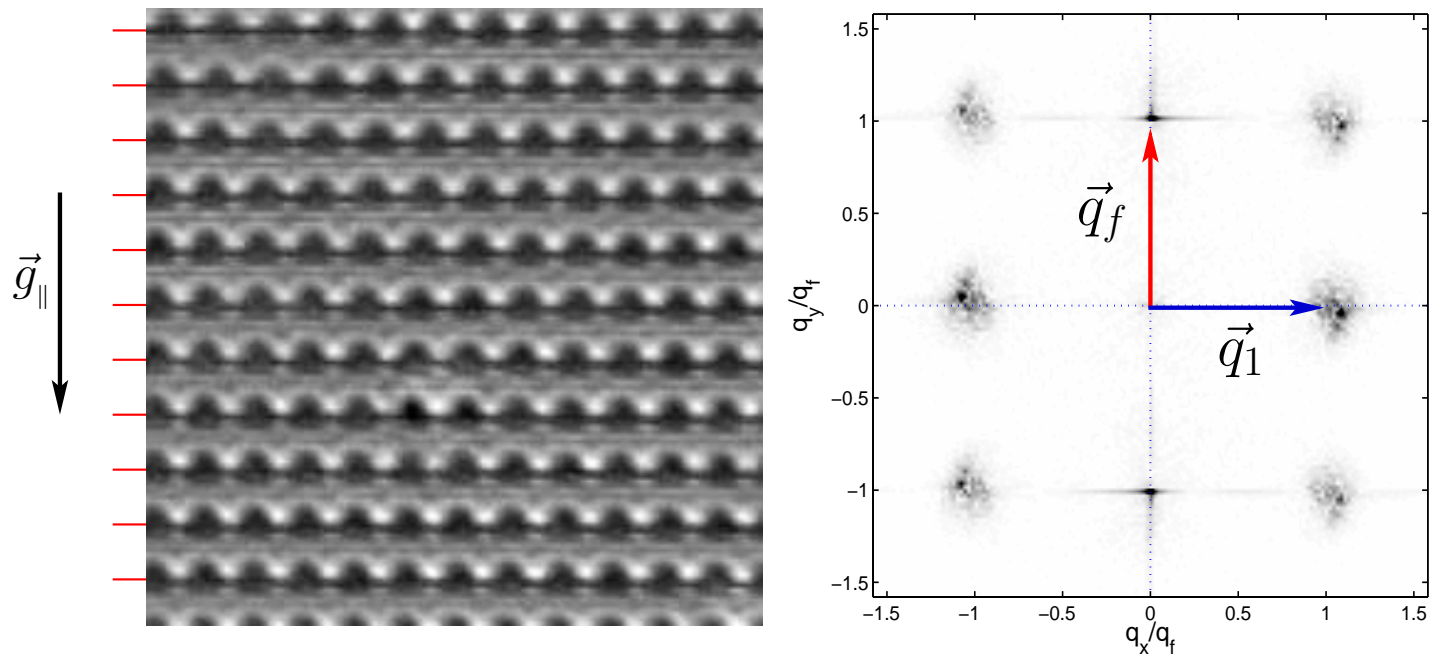

Figure 4.32: Bimodal pattern and its corresponding Fourier transform (taken at $\left.\gamma=55^{\circ}, \varepsilon=0.11\right)$. Red stripes mark the location of the forcing structure.

from $\left|\vec{q}_{1,2}\right|=0.83 q_{f}$ to $0.94 q_{f}$ (red points in right plot of Fig. 4.31). This is due to a monotonic increase of the x-component from $q_{x_{1,2}}=0.66 q_{f}$ at $\varepsilon=0.038$ to $0.80 q_{f}$ at $\varepsilon=0.155$ where CR starts to set in (Fig. 4.31e). At the same time the y-component of $\vec{q}_{1}$ and $\overrightarrow{q_{2}}$ stays constant at $q_{y}=0.5 q_{f}$ for all $\varepsilon$ at which HR exist.

This leads to a change in angle (with respect to $\vec{q}_{f}$ ) for those modes from $\alpha=53^{\circ}$ at HR onset $(\varepsilon=0.038)$ to $\alpha=58^{\circ}$ at onset of $\mathrm{CR}\left(\varepsilon=0.155^{\circ}\right)$.

Here again $\vec{q}_{1}$ and $\vec{q}_{2}$ are slightly asymmetric with respect to the y-axis due to a small circular horizontal flow. Therefore, for the values given in this section, the average of mode $\vec{q}_{1}$ and $\vec{q}_{2}$ were calculated.

\subsubsection{Bimodal Pattern}

For inclination angles $\gamma>33^{\circ}$ one observes an instability of the rolls to a Bimodal Pattern (BM). One mode is given by the forcing wave vector $\vec{q}_{f}$ and the second one is perpendicular to $\vec{q}_{f}$ as shown in Fig. 4.32. The bimodal pattern is not due to a simple superposition of two roll patterns as they appear at a first glance. Instead, the phase of the forced longitudinal rolls is disturbed periodically and a pattern occurs similar to drops sitting on a rectangular lattice. As can be seen in Fig. 4.22, the area where bimodal pattern exist in forced ILC phase diagram is much larger as for unforced ILC and extended to the left side - to smaller inclination angles.

The main modes of the bimodal pattern are shown in the Fourier spectra in Fig. 
4.32:

$$
\vec{q}_{f}=\left(\begin{array}{c}
0 \\
q_{f}
\end{array}\right) \quad \text { and } \quad \vec{q}_{1} \approx 1.1\left(\begin{array}{c}
q_{f} \\
0
\end{array}\right) .
$$

In fact, the length of the side mode $\vec{q}_{1}$ increases with $\varepsilon$ as shown in the inlet of Fig. 4.33. Here, for an inclination angle of $\gamma=55^{\circ}$, the side mode length grows from $\left|\vec{q}_{1}\right|=1.02 q_{f}$ at onset of bimodal pattern $(\varepsilon=0.06)$ to $1.17 q_{f}$ at $\varepsilon=0.3$.

Figure 4.33 shows the evolution of the squared amplitude of the two modes in the Fourier spectra (or the amplitude in the power spectra). Plotting the evolution of the amplitude of the two main modes, reveals clearly the onset of the bimodal instability at $\varepsilon=0.07$. Until the onset of BM, the amplitude of the forced mode grows as expected following the cubic amplitude equation (imperfect bifurcation) as shown in Fig. 4.2. At $\varepsilon=0.07$, the amplitude of the forced mode stops growing and the side mode occurs in a forward bifurcation. The squared of the amplitude can be fitted by a linear curve and thus one can say, that for the longitudinal mode $\left(\vec{q}_{1}\right)$ a pitch fork bifurcation takes place at the onset of bimodal pattern.

As already mentioned, the bimodal pattern can not be explained by a simple superposition of two roll patterns. In the Fourier spectra in Fig. 4.32 two other peaks corresponding to $\vec{q}_{3}=\vec{q}_{f}+\vec{q}_{1}$ and $\vec{q}_{4}=\vec{q}_{f}-\vec{q}_{1}$ are visible as well. Thus, it is very likely that beside the amplitude, also the phase of the forced rolls is modulated periodically. One can write a formula which describes the pattern as follows:

$$
I_{B M}=A_{0} \cos \left(\overrightarrow{q_{f}} \vec{r}+A_{1} \cos \overrightarrow{q_{1}} \vec{r}\right)+A_{2} \cos \left(\overrightarrow{q_{1}} \vec{r}\right) .
$$

A calculated pattern regarding Eq. 4.10 is shown in Fig. 4.34. The amplitudes are:

$$
A_{0}=4, \quad A_{1}=0.5, \quad A_{2}=-2
$$

A very similar pattern was also found and described in centrifugal driven cylindrical convection by Brausch [51] and Auer et. al. [91].

Dynamic is introduced in the bimodal pattern by defects. While the forced mode is free of any defects, in the side mode $\vec{q}_{1}$, dislocations appear and disappear on a short time scale and are accompanied by a localized area of reduced amplitude. For inclination angles $\gamma<50^{\circ}$ the number of dislocations increases with increasing Rayleigh number and the bimodal pattern turn continuously into crawling rolls.

For inclination angles $\gamma>50^{\circ}$ one sees a different behavior. Dislocations start to cluster and rearrange themselves to form a new state of scepter pattern. 


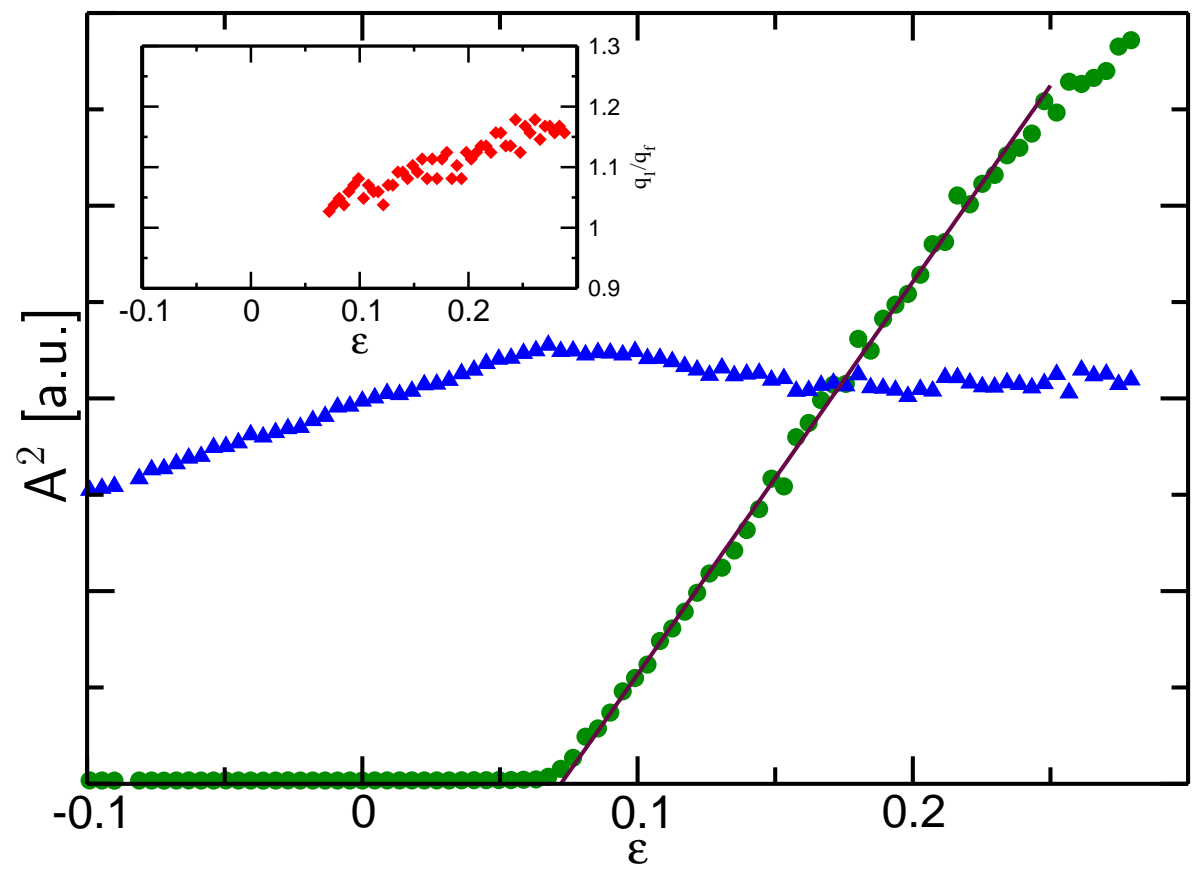

Figure 4.33: Mode evolution of bimodal pattern. Shown are the evolution of the squared amplitudes of $\vec{q}_{f}(\boldsymbol{\Delta})$ and of $\vec{q}_{1}(\bullet)$ for increasing $\varepsilon$. The straight line is a linear fit of the green points. The inlet shows the evolution of the length of the instability vector $\left|\vec{q}_{1}\right|$. Experiments were taken at $\gamma=55^{\circ}$.

Figure 4.34: Bimodal pattern, calculated with Eq. 4.10. The amplitudes are: $A_{0}=4, A_{1}=0.5$ and $A_{2}=-2$.

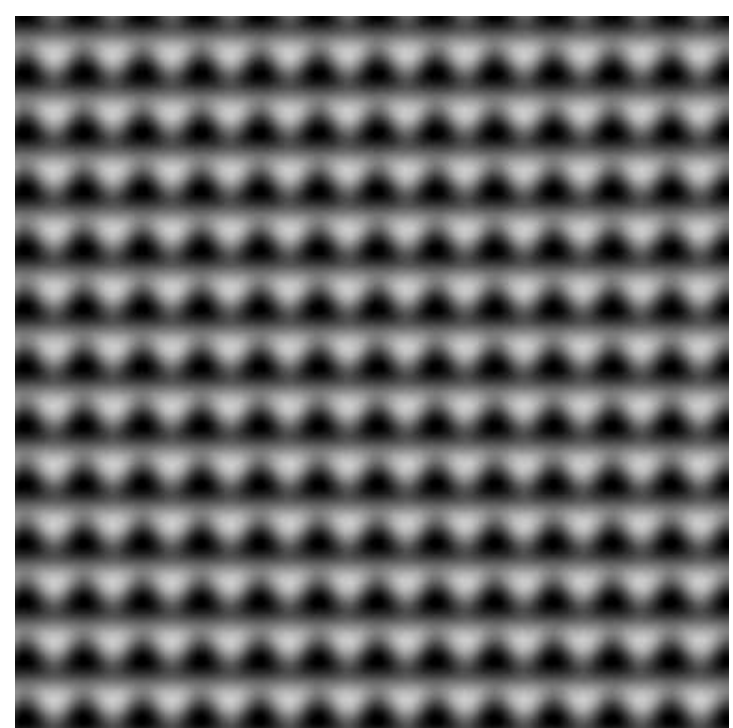




\subsubsection{Scepter Pattern}

For large inclination angles $\left(50^{\circ}<\gamma<75^{\circ}\right)$ and large temperature differences $(\varepsilon>0.2)$ the bimodal pattern do not turn into phase turbulent crawling rolls immediately, but first show a superlattice pattern (shown in Fig. 4.35). Superlattice patterns are patterns that have spatial structure on more than one length scales. Two or more discrete sublattices interact and form a superlattice. Such patterns were observed already in several different pattern forming systems, usually when time periodic forcing was present [93, 63, 94, 95, 96].

In our case, a complex structure similar to a "Y" or a scepter is located at every lattice point at a rectangular lattice. This rectangular lattice has a unit length in x-direction, which is slightly shorter than the forcing wave length $\left(\approx 0.88 \lambda_{f}\right)$, while in $\mathrm{y}$-direction it shows a periodicity of $2 \lambda_{f}$.
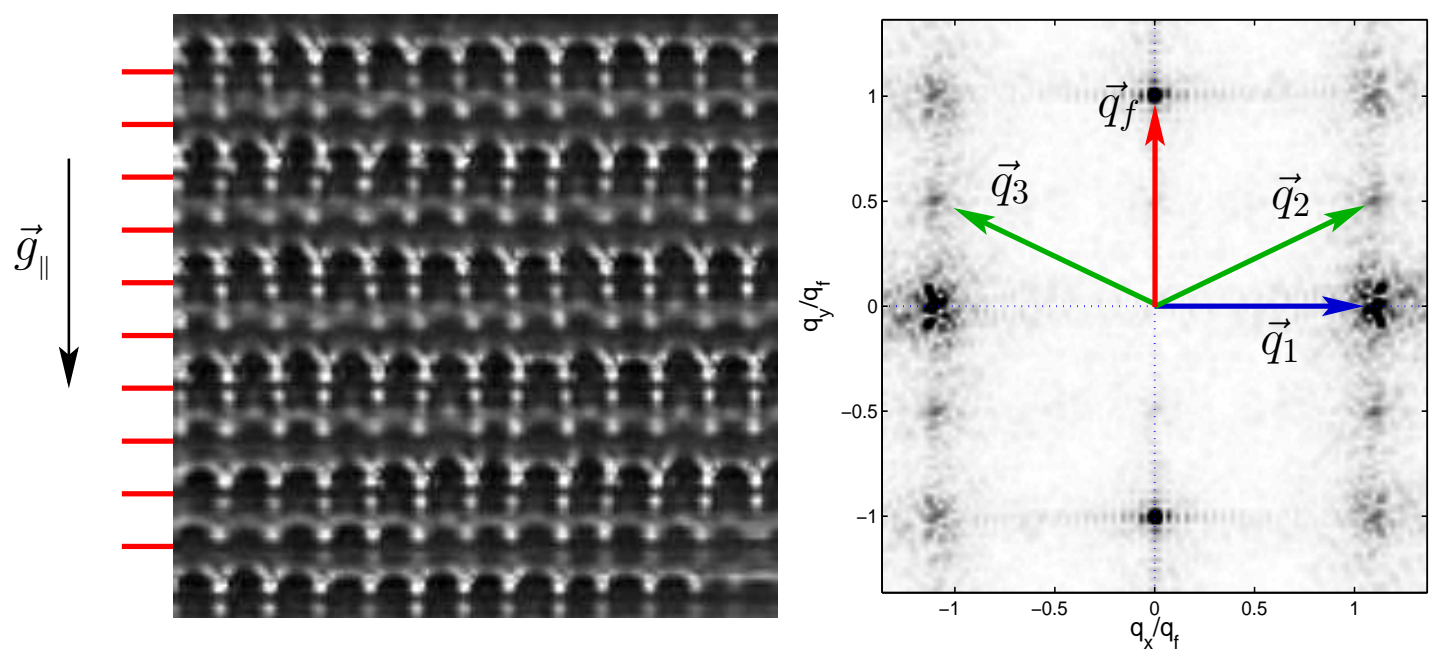

Figure 4.35: Raw image and Fourier transform of scepter pattern, taken at $\gamma=72.5^{\circ}$ and $\varepsilon=0.32$. Red stripes mark the bottom plate texture.

Scepter patterns and their corresponding Fourier transform are shown in Fig. 4.35. The Fourier transform reveals the four underlying modes that build up the two sublattices. One can consider a first rectangular lattice build by the wave vectors:

$$
\vec{q}_{f}=q_{f}\left(\begin{array}{l}
0 \\
1
\end{array}\right) \quad \text { and } \quad \vec{q}_{1}=q_{f}\left(\begin{array}{c}
1.14 \\
0
\end{array}\right) .
$$

The second lattice can be considered as build by the two symmetric modes:

$$
\vec{q}_{2}=q_{f}\left(\begin{array}{c}
1.14 \\
0.5
\end{array}\right) \quad \text { and } \quad \vec{q}_{3}=q_{f}\left(\begin{array}{c}
-1.14 \\
0.5
\end{array}\right)
$$


Also here a resonant triad occurs:

$$
\overrightarrow{q_{f}}=\overrightarrow{q_{2}}+\overrightarrow{q_{3}}
$$

The scepter pattern can also be seen as a convolution of a single scepter and a 2-dimensional delta array (Fig. 4.36) with lattice vectors:

$$
\vec{a}_{1}=\lambda_{f} \cdot\left(\begin{array}{c}
0.88 \\
0
\end{array}\right) \text { and } \quad \vec{b}_{2}=\lambda_{f} \cdot\left(\begin{array}{l}
0 \\
2
\end{array}\right)
$$
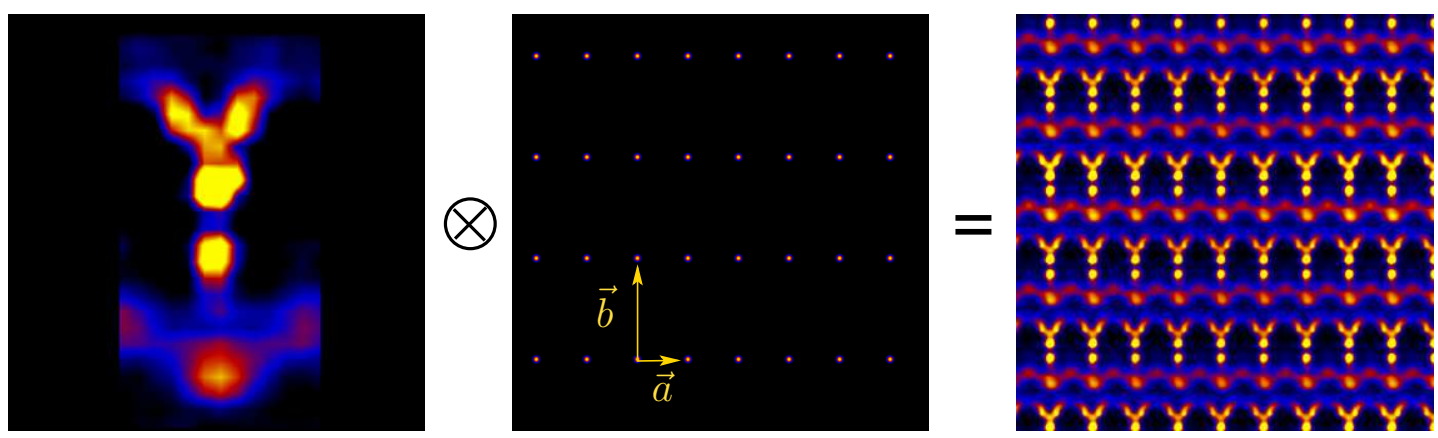

Figure 4.36: Scepter pattern can be seen as a convolution of a single Scepter (left) and a two dimensional $\delta$-array with lattice vectors $\vec{a}$ and $\vec{b}$. The picture on the right is a convolution of the first two and not an experimental result.

If $\vec{a}$ and $\vec{b}$ are the lattice vectors in space, than $\vec{a}^{\prime}=2 \pi \frac{\vec{a}}{|\vec{a}|^{2}}$ and $\vec{b}^{\prime}=2 \pi \frac{\vec{b}}{|\vec{b}|^{2}}$ are the reciprocal lattice vectors, meaning the lattice vectors that build the $\delta$-array in the Fourier space. In fact it is $\vec{a}^{\prime}=\vec{q}_{1}$. A mode that corresponds to the second vector $\vec{b}^{\prime}=\vec{q}_{f} / 2$ is also visible in Fig. 4.35, even though its amplitude is very low. One can build $\overrightarrow{b^{\prime}}$ by a linear combination of the basis vectors:

$$
\overrightarrow{b^{\prime}}=\overrightarrow{q_{2}}-\overrightarrow{q_{1}}
$$

It is important to note that $\overrightarrow{b^{\prime}}$ is equal to the instability vector $\vec{q}_{1}$ of the Transverse Bursts (Fig. 4.13). In addition, TB and scepter pattern occur in similar regions of the $\gamma-\varepsilon$ phase space and therefore, one can assume that both modes are expressions of the same instability. Only here, the pattern itself is stabilized by the forcing. Therefore, one can see the scepter pattern as a stabilized version of the transverse bursts.

Scepter pattern usually never cover the whole cell but only about $50 \%-60 \%$ of it. The rest of the cell is still covered with bimodal pattern, described in the previous section. Due to fluctuations the scepters turn back to bimodals pattern, 
while at other areas of the cell, the bimodal turn into scepter. For higher $\varepsilon$ a continuous transition to a chaotic state occurs.

Scepter pattern have a local lifetime of $\tau \approx 20-50 t_{\kappa}$ in dependence on $\varepsilon$ and $\gamma$. Therefore, they are not just a transient state as Transverse Bursts, but a persisting pattern. SP get destroyed once in a while (every $20-50 t_{\kappa}$ ) by fast traveling defects. These defects cluster together and form an area where no scepter occur for a short while. On short time scales SP show a non-periodic dancing-like behavior, whereby they are either squeezed together or stretched apart. In addition the top of each scepter wiggles with a high frequency $\left(>1 / t_{\kappa}\right)$ to the left and to the right (movie available on enclosed CD-ROM).

The evolution of the three main modes $\vec{q}_{f}, \vec{q}_{1}$ and $\vec{q}_{2}$ is shown in Fig. 4.37. Starting at $\varepsilon=0$ the amplitude of the forced mode grows following an imperfect bifurcation $(+)$ as already shown in Fig. 4.33. At $\varepsilon=0.07 \mathrm{BM}$ set in and the mode $\vec{q}_{1}$ follows a pitchfork-like bifurcation, whereas the amplitude of $\vec{q}_{f}$ reaches a plateau. At $\varepsilon=0.3$ the third mode $\overrightarrow{q_{2}}$ occurs and its amplitude increases slightly, while the amplitude of the other modes decreased at onset of $\vec{q}_{2}$. Unfortunately here, the shadowgraph focuses to close to the first caustic and is not anymore in the regime, where its intensity goes linear with the convection amplitude. Therefore, one cannot make conclusions from the shadowgraph signal with respect to the mode amplitude for $\varepsilon>0.3$.

What are the underlying modes of the scepter patterns and how do they interact in order to form that superlattice structure? One of course knows, that the basic modes are $\vec{q}_{f}, \vec{q}_{1}$ and $\vec{q}_{2}$. But in order to derive an amplitude equation what will not be done here - one needs to know how they interact and where the amplitude and where the phase is modulated. A good way of analysis is to consider only certain modes in the raw picture and remove all the rest. Two effects complicates the whole analysis. The first thing is the shadowgraph setting. Since the shadowgraph was usually set in a way, that it focuses far away from the first caustic for small convection strength, for stronger convection (large $\varepsilon$ ) the axial distance at which the shadowgraph intensity becomes maximal $\left(z_{\max }\right)$ moves down to smaller $z$ and comes closer to the image plane of the shadowgraph. Therefore, the shadowgraph intensity is no longer proportional to the temperature distribution in the fluid. Instead, one sees small areas with high light intensity and large areas with low light intensity, resulting in an higher intensity of the higher harmonics.

A second problem occurs due to the optical effect of the SU-8 texture to the shadowgraph image, since that effect depends also on the convection strength itself and cannot be completely removed by background division as explained in the experimental chapter (Section 3.3.2). Figure 4.38a shows a representative section of the cell of length $14 \lambda_{f}$ at $\gamma=72.5^{\circ}$ and $\varepsilon=0.37$. It shows large areas of scepter pattern but also small areas where no SP exist, but only some small 


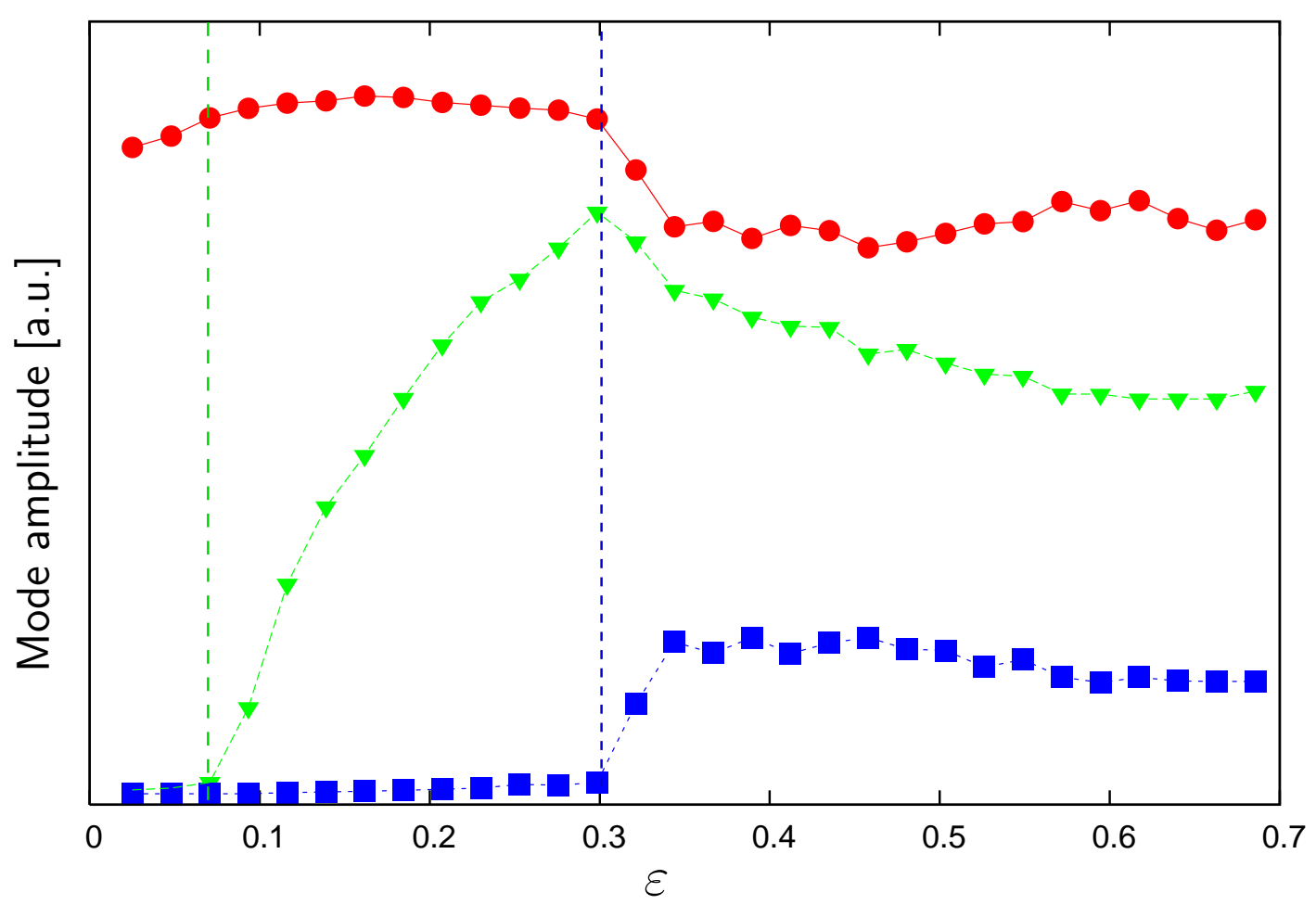

Figure 4.37: Amplitude development during the occurrence of scepter pattern for the forced mode $\vec{q}_{f}(+)$, the bimodal mode $\vec{q}_{1}(\times)$ and the additional scepter mode $\vec{q}_{2}(\star)$. (taken at $\gamma=72.5^{\circ}$ ). The dashed lines mark the onset of the bimodal pattern (green) and the onset of the scepter pattern (blue).

amplitude bimodals. The red lines at the left shows the location of the SU-8 ridges. Hence the distance between two red lines is exactly $\lambda_{f}$. From this area no light comes into the camera and black lines appear in the image. By analyzing the image and if one tries to reproduce the pattern, one has to take into account that the black stripes in the image are not necessarily part of the pattern, but might be only an artefact of the shadowgraph technique. In Fig. 4.38b the Fourier spectra of Fig. 4.38a is shown. Again, the amplitude of the mode with vector $\vec{q}_{f} / 2$ can hardly be seen. But on the other hand the higher mode $\frac{3}{2} \vec{q}_{f}$ is visible. Figure $4.38 \mathrm{~b}$ only shows the central part of the Fourier transform. The amplitudes of the higher harmonics of $\vec{q}_{f} / 2$ which are not shown in the Fourier transform are:

\begin{tabular}{|l|c|c|c|c|c|c|}
\hline Mode: & $0.5 \vec{q}_{f}$ & $\vec{q}_{f}$ & $1.5 \vec{q}_{f}$ & $2 \vec{q}_{f}$ & $2.5 \vec{q}_{f}$ & $3 \vec{q}_{f}$ \\
\hline Amplitude: & 0.20 & 1 & 0.47 & 0.38 & 0.38 & 0.42 \\
\hline
\end{tabular}

As can be seen, the amplitudes of the higher harmonic modes do not decrease 
monotonically, but stays at a constant level. Now, in order to get more information one can remove several modes out of the raw image (Fig. 4.38a) and looks, which modes are the important one to build a scepter pattern. This is done by a simple Fourier filtering, where the modes which should be removed are set to zero in the Fourier transform and the resulting image is transformed back into real space.

By using only the modes in y-direction up to $1.5 q_{f}$ (green surrounded area in Fig. $4.38 \mathrm{~b}$ ) and removing all the other modes, one gets the image shown in Fig. 4.38c. One sees a superposition of the two modes $\left(q_{f}\right.$ and $\left.1.5 q_{f}\right)$ at those places where scepters exists, while at other places (e.g. at the middle of the left half) only one mode is visible, the forced mode. This image also shows nicely that sometimes defects exist at which the scepters are shifted by $\lambda_{f}$ in y-direction (red circle in Fig. 4.38c). One can build a pattern shown in Fig. $4.38 \mathrm{c}$ in the following way:

$$
I_{S P 0}=A_{1} \cdot\left(\cos \left(\vec{q}_{f} \vec{r}\right)+0.5 \sin \left(1.5 \vec{q}_{f} \vec{r}\right)\right) .
$$

The factor of 0.5 in the second term was chosen according to the amplitude of the second harmonic in Fig. 4.38b.

Focusing only on the modes in x-direction and small $q_{y}$ (red rectangle in Fig. $4.38 \mathrm{~b}$ ) and removing all other modes produces the image in Fig. 4.38d. The lines in y-direction are only slightly bended but otherwise well aligned due to gravity. At the left side of the image one sees coherent phase defects resulting in a destruction of scepters at that area.

Figure $4.38 \mathrm{e}$ again shows the reproduction of the pattern by using only modes with a y-component of $q_{y}= \pm 0.5 q_{f}$ (blue framed area in Fig. 4.38b. The zig-zag pattern can be produced by rolls with a wave length of $2 \lambda_{f}$ and phase modulations with a wave number of $1.14 q_{f}$ (which is the x-component of $\overrightarrow{q_{2}}$ as stated above. It is clear, that this mode is very essential. A pattern as in Fig. 4.38e can be written by a formula like for undulations as in Eq. 4.5:

$$
I_{S P 1}=A_{1} \cos \left(0.5 \vec{q}_{f} \vec{r}+P_{1} \cos \left(\overrightarrow{q_{1}} \vec{r}\right)\right) .
$$

Image (f) now uses all the modes with a y-component of $-0.75 q_{f}<q_{y}<0.75 q_{f}$, meaning the modes used for image (d) and (e) together (brown area in Fig. $4.38 \mathrm{~b}$ ). After setting all other modes to zero and calculating the inverse Fourier transform, the image one gets now has astonishing similarities with transverse bursts shown in Fig. 4.12.

Now, let us try to write down an equation for scepter pattern. The basis will be a zig-zag pattern with a basic wave vector $\vec{q}_{f}$ as given in Eq. 4.12. The corresponding gray image is shown in Fig. 4.39a. In addition one uses a similar zig-zag pattern, but with a $\vec{q}_{f}$ as basic wave vector and with a smaller phase modulation:

$$
I_{S P 2}=A_{2} \cos \left(\vec{q}_{f} \vec{r}+P_{2} \cos \left(\vec{q}_{1} \vec{r}\right)\right) .
$$



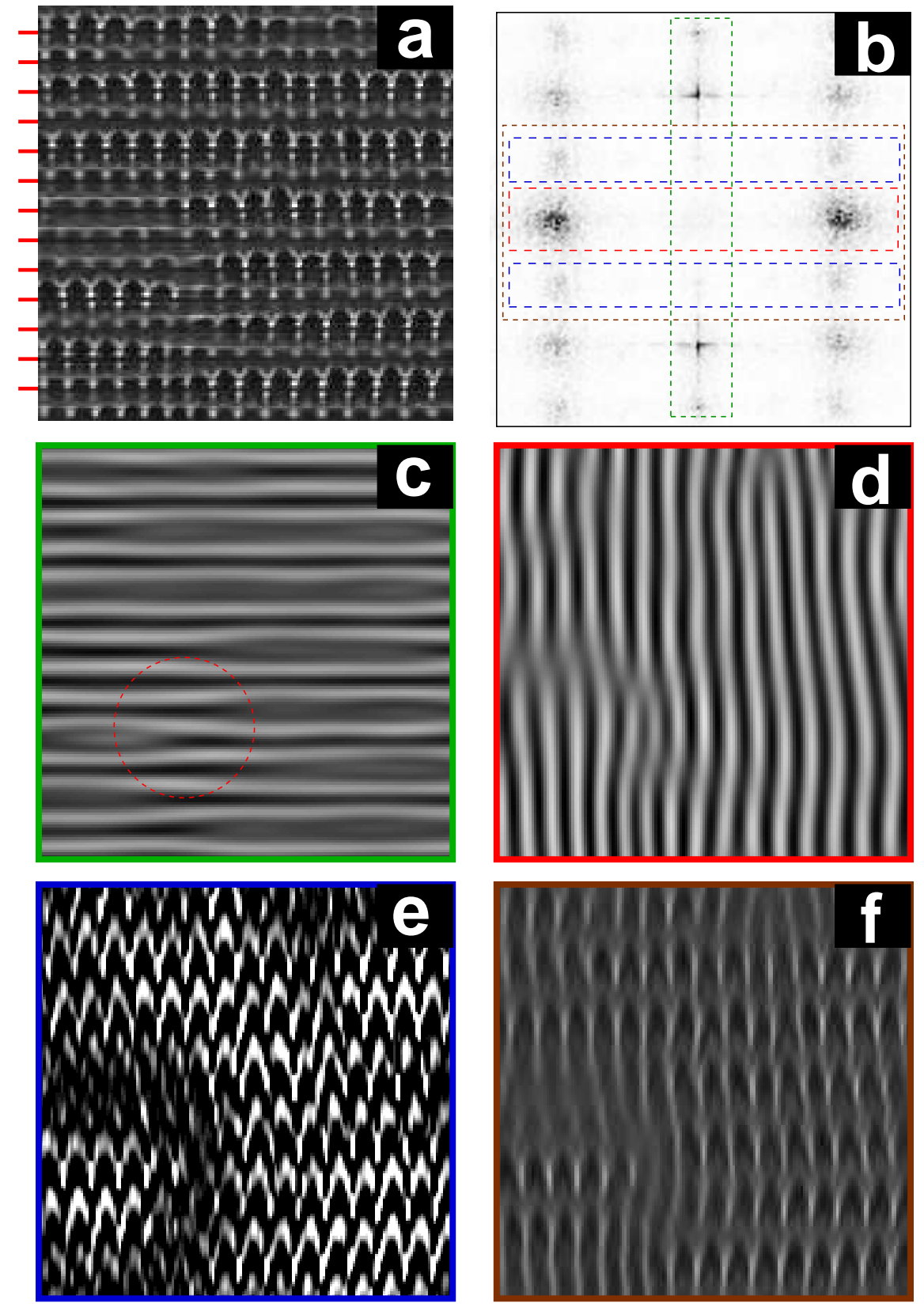

Figure 4.38: Fourier decomposition of scepter pattern. (a): Section with scepter pattern. (b): Fourier spectra. (c-f): Different Fourier filtered versions of (a) where only certain modes are used and the rest are set to zero. The modes which were used to rebuild the pattern are marked with color code in (b). 
As third, one takes a vertical stripe pattern with $\overrightarrow{q_{1}}$ and imposes onto it a modulation of the stripe amplitude in vertical direction with $\vec{q}_{f}$ (Fig. 4.39c):

$$
I_{S P 3}=A_{3} \cos \left(\vec{q}_{1}\right) \cdot\left(P_{3} \cos \left(0.5 \cdot \overrightarrow{q_{f}} \vec{r}\right)-1\right) .
$$

An approximation of the scepter pattern is shown in Fig. 4.39d, which is given by:

$$
I_{S P}=I_{S P 1}+I_{S P 2}+I_{S P 3}
$$

Note that this Formula includes the formula for bimodal pattern (Eq. 4.10) if one sets $A_{1}=P_{3}=0$.

A calculated Intensity regarding Eq. 4.15 and with

$$
A_{1}=2, \quad P_{1}=0.8, \quad A_{2}=2, \quad P_{2}=0.8, \quad A_{3}=1, \quad P_{3}=1
$$

is shown in Fig. 4.39d. One can compare the result with a scepter pattern that was generated by a convolution of a single scepter and a delta array (Fig. 4.39e). The main structures is similar in both cases, but it is also clear, that one needs also higher order modes to produce very sharp structures and structures with length lower than its periodicity.

\subsubsection{Heart Pattern}

A further increase in inclination angle $\left(\gamma>75^{\circ}\right)$ leads to a stronger shear flow and the occurring shear instability is dominant over the buoyancy driven instability. This leads to an instability of the forced transverse rolls even before convection sets in in the reference cell.

The instability leads to a heart pattern (HP) as shown in Fig. 4.40. The basic modes for this pattern are:

$$
\vec{q}_{1}=q_{f}\left(\begin{array}{c}
0.5 \\
0.5
\end{array}\right) \quad \text { and } \quad \vec{q}_{2}=q_{f}\left(\begin{array}{r}
0.5 \\
-0.5
\end{array}\right) \text {. }
$$

Again the system uses these modes in order to resonate with the forcing mode:

$$
\overrightarrow{q_{f}}=\overrightarrow{q_{1}}+\overrightarrow{q_{2}}
$$

One can see the heart pattern as single heart sitting on a square lattice which is inclined $45^{\circ}$ with respect to the gravity component. Or, in other words, it is a convolution of a single heart with a square $\delta$-array with lattice vectors:

$$
\vec{a}=2 \pi \frac{\overrightarrow{q_{1}}}{q_{1}^{2}} \quad \text { and } \quad \vec{b}=2 \pi \frac{\overrightarrow{q_{2}}}{q_{2}^{2}}
$$



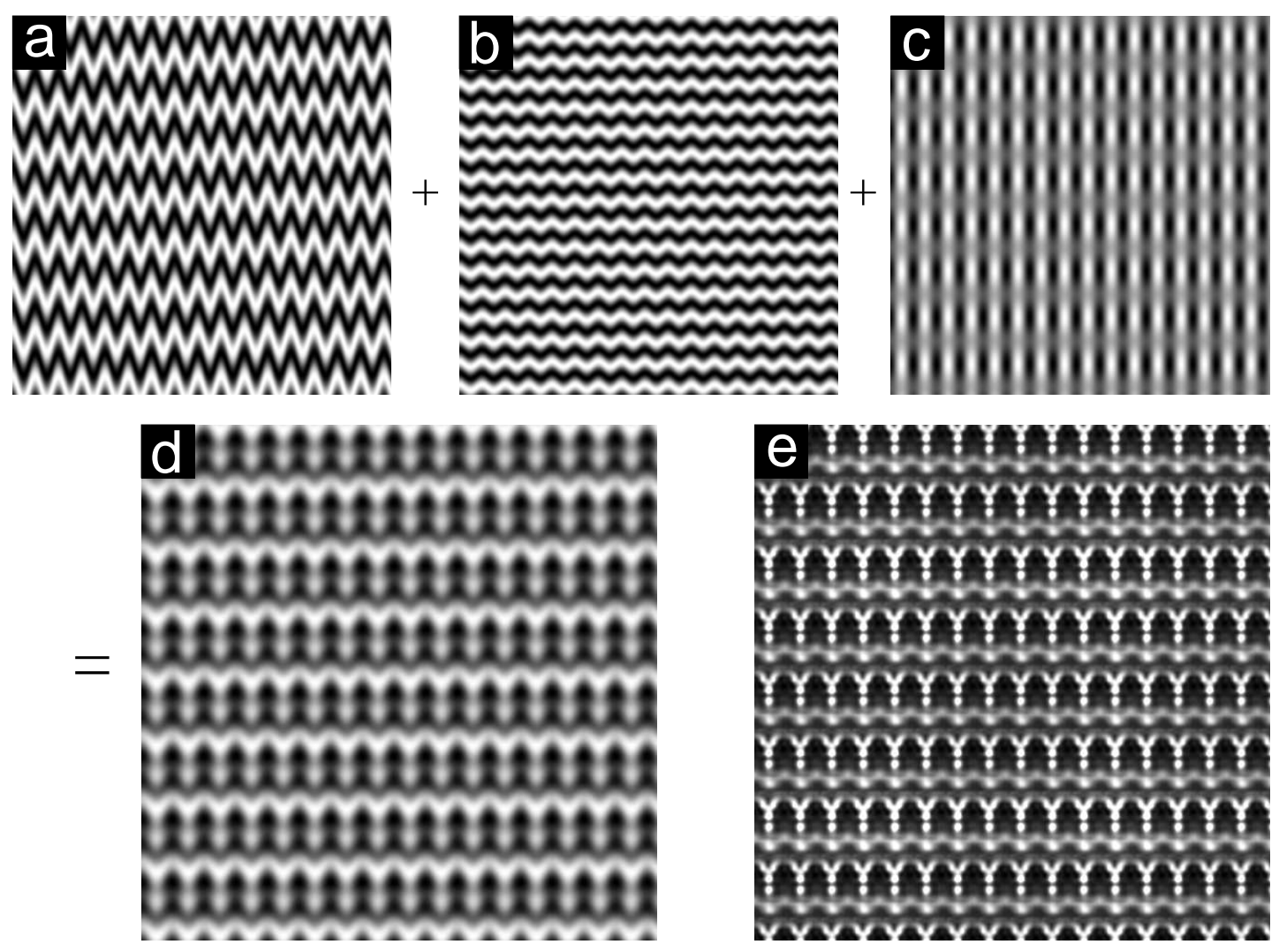

Figure 4.39: Calculated scepter pattern by using Eq. 4.15. Zig-zag pattern with $0.5 \vec{q}_{f}$ as basic vector (a), bimodal pattern (b) and zig-zag pattern with basic wave vector $\vec{q}_{f}$ (c) result in a scepter-like pattern (d). For comparison the scepter pattern, created by convolution (see Fig. 4.36) is shown in (e).

This is illustrated in Fig. 4.41. The right image shows the result of a computer generated convolution between the lattice and the single heart.

Heart patterns show a very slow drift and in addition large scale amplitude and phase modulations. Phase defects occur along this modulation and are aligned with the axis of the underlying lattice. In this way the heart pattern exist in domains, which are separated by these defect lines. HP are in this respect very similar to Switching Diamond Panes (Fig. 4.42) which occur in inclined layer convection without forcing at inclination angles larger than $90^{\circ}[49,51]$.

\subsection{Forcing Oblique Rolls: $\varphi=60^{\circ}$}

The last section of this chapter deals with experiments in which rolls were forced with axis aligned $\varphi=60^{\circ}$ to the in-plane gravity component. The phase diagram 

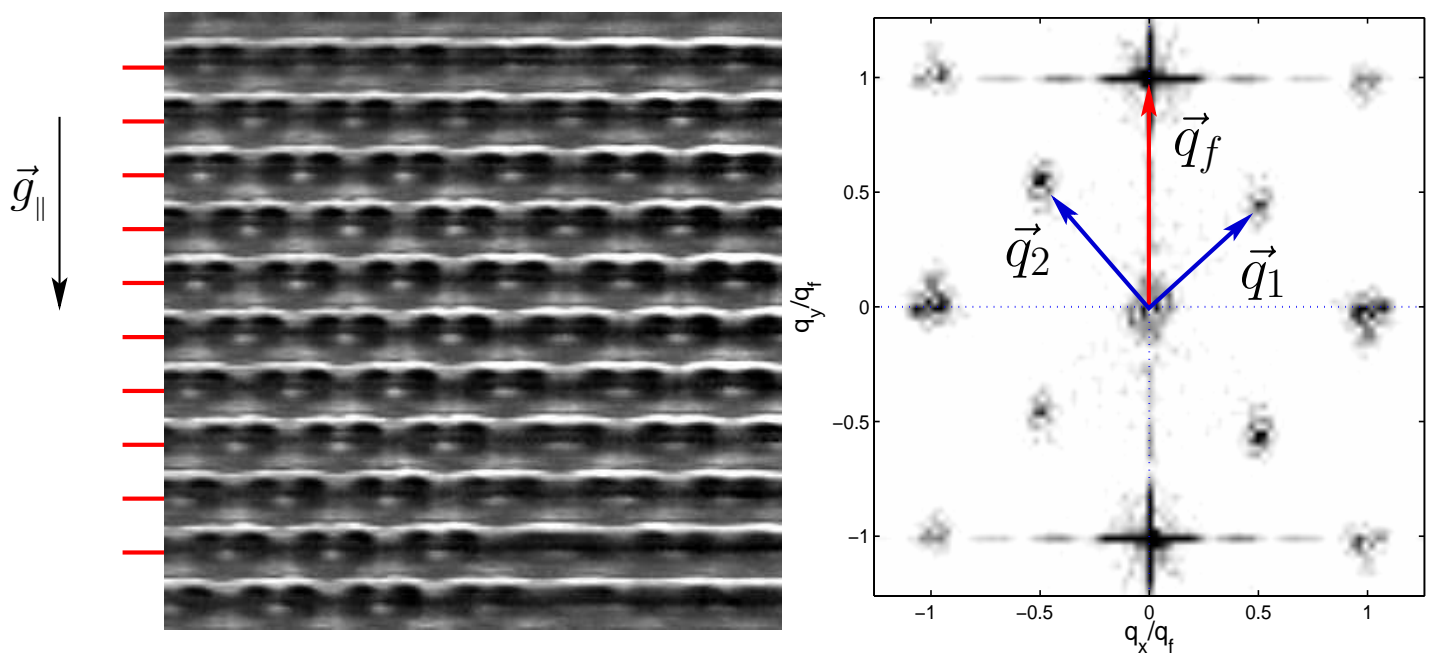

Figure 4.40: Heart pattern and first modes of its Fourier spectrum at $\gamma=90^{\circ}$ and $\varepsilon=0.32$. Red stripes mark the bottom plate texture.
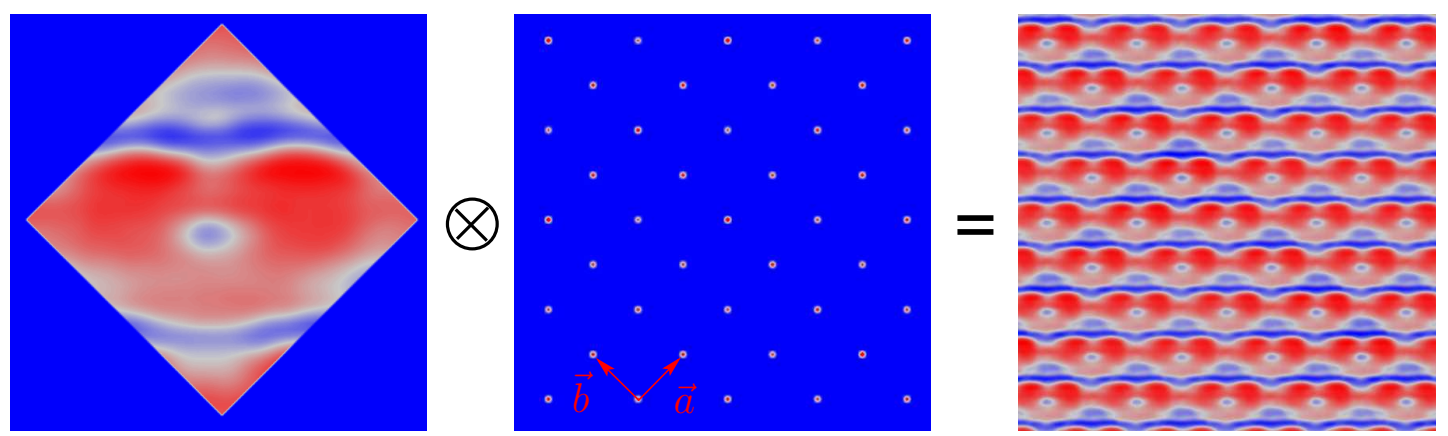

Figure 4.41: Heart pattern can be seen as a convolution of a single heart and squared delta array which is inclined by $45^{\circ}$. The right image shows a computer generated convolution of the left and the middle image. 

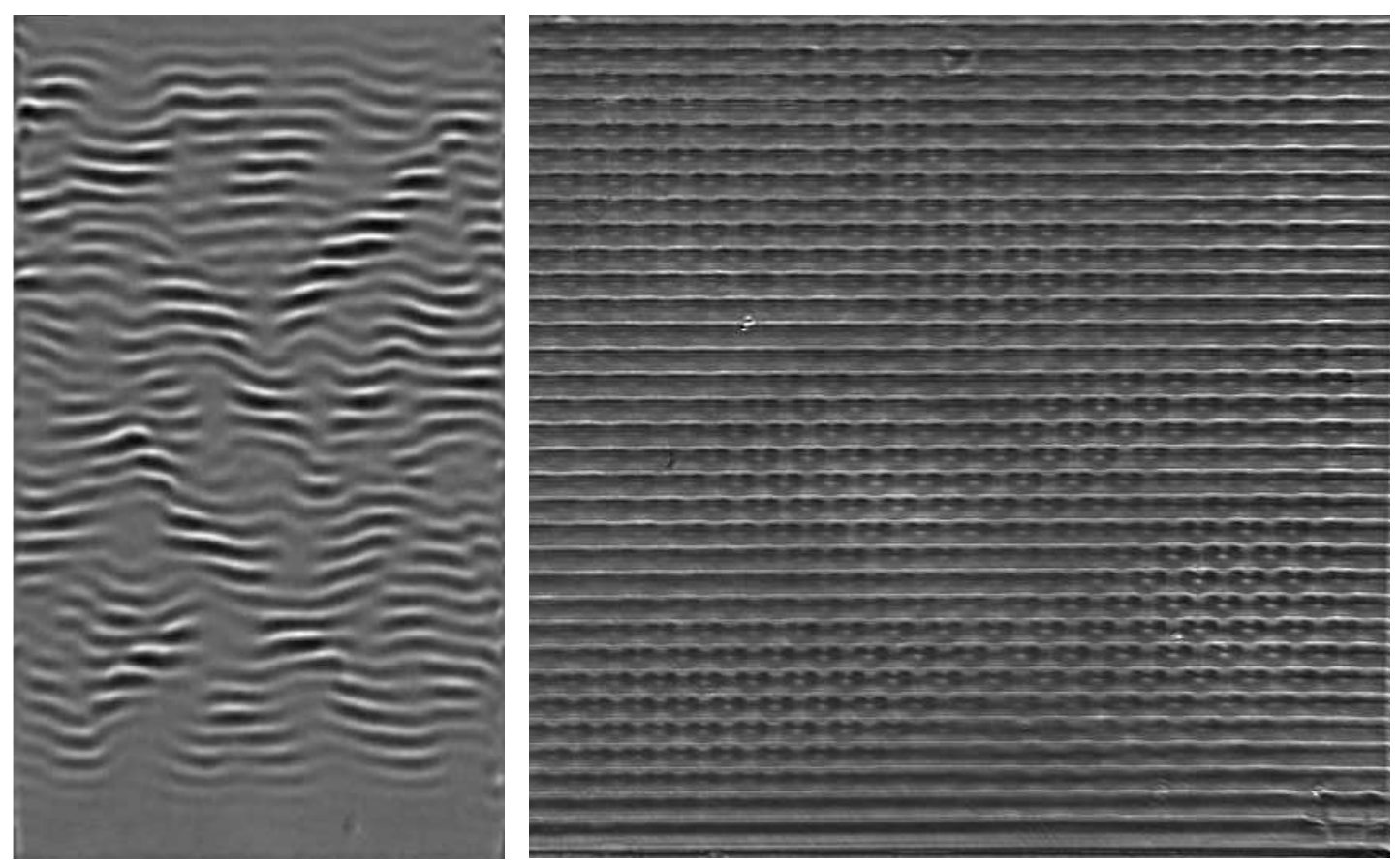

Figure 4.42: Switching diamond panes (left) at $\gamma=100^{\circ}$ and $\varepsilon=0.1$ (from [48]) and heart pattern (right) at $\gamma=90^{\circ}$ and $\varepsilon=0.32$. In both patterns, obliquely aligned areas with strongly reduced amplitude are characteristic.

of the $\gamma-\varepsilon$ phase space shows the different patterns observed. Note that the left part of the phase diagram has a logarithmic scaled $\varepsilon$-axis, in the same way as in previous cases. For $\gamma>75^{\circ}$ a linear scale was chosen, since here interesting patterns occur already for negative $\varepsilon$.

Stable forced oblique rolls (OR) are observed for low $\varepsilon$. These rolls are again in phase with the SU-8 texture and therefore have wave vectors:

$$
\vec{q}_{f}=q_{f}\left(\begin{array}{c}
-\cos \left(60^{\circ}\right) \\
\sin \left(60^{\circ}\right)
\end{array}\right)
$$

When compared to the two preceding scenarios, the instability line of OR lies below the instability line of LR in the $\varphi=0^{\circ}$ case (Fig. 4.3) and slightly above the instability line of $\mathrm{TR}$ in the $\varphi=90^{\circ}$ case (Fig. 4.22). In addition, the minimum value of the instability line is shifted to higher inclination angles, and is now located at $\gamma \approx 35^{\circ}$. These observations are in accordance with the maximum of the onset curve of the corresponding $60^{\circ}$-oblique rolls for unforced ILC (dashed violet line in Fig. 4.43). The maximum of the onset curve for rolls with different orientations $\varphi$ also shifts to larger inclination angles $\gamma$ if the orientation angle $\varphi$ decreases (compare Fig. 2.2). 

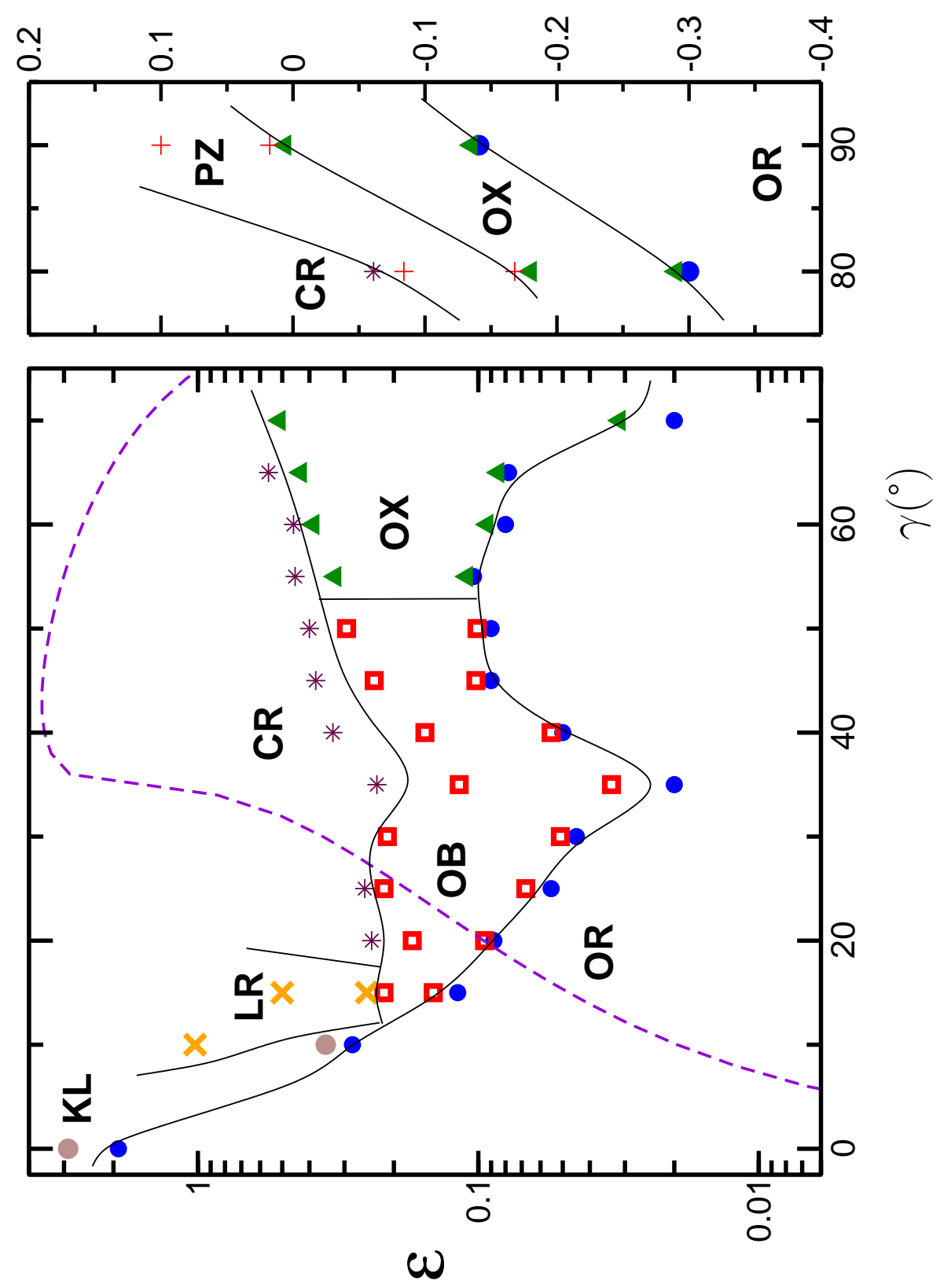

惫富高焉

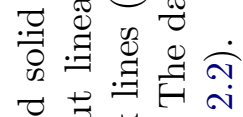

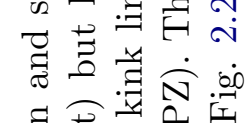

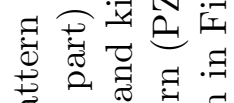

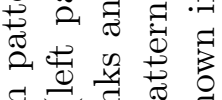

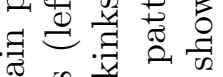

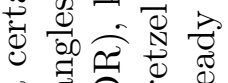

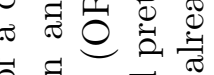

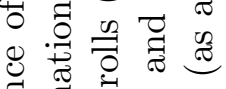

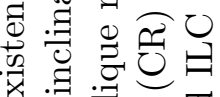

잉

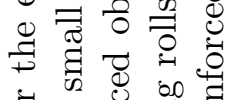

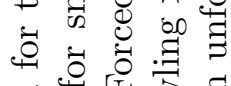

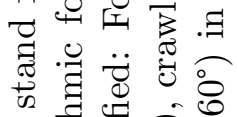

on 要表

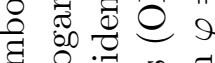

की

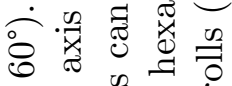

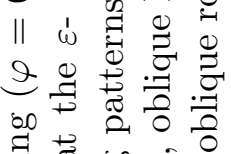

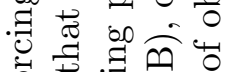

응

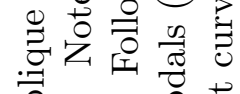

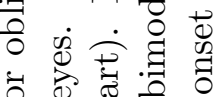

훙

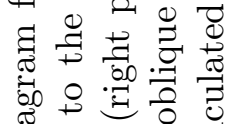

0 कै

每

궁

$\ddot{0}$

+

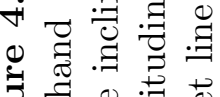

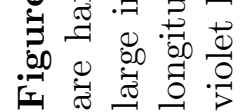


For small inclination angles $\left(\gamma<10^{\circ}\right)$ the forced oblique rolls become unstable to a coherent kink line (KL) structure (• in Fig. 4.43), similar to the state found for the parallel forcing case (Fig. 4.44a). Also in this case, KL accumulate to form larger patches of kink clusters. A further increase in $\varepsilon$ leads to a stronger shear flow which forces the kink clusters to align parallel to gravity, resulting in modulated longitudinal rolls (LR) ( $\times$ in Fig. 4.43) as can be seen in Fig. 4.44b. A similar longitudinal alignment tendency was reported in the occurrence of $\mathrm{RO}$, in the transverse forcing scenario (Sec. 4.3). Also here, the rolls are not exactly parallel to gravity but slightly oblique with an angle of approximately $8^{\circ}$ (averaged over the whole cell).
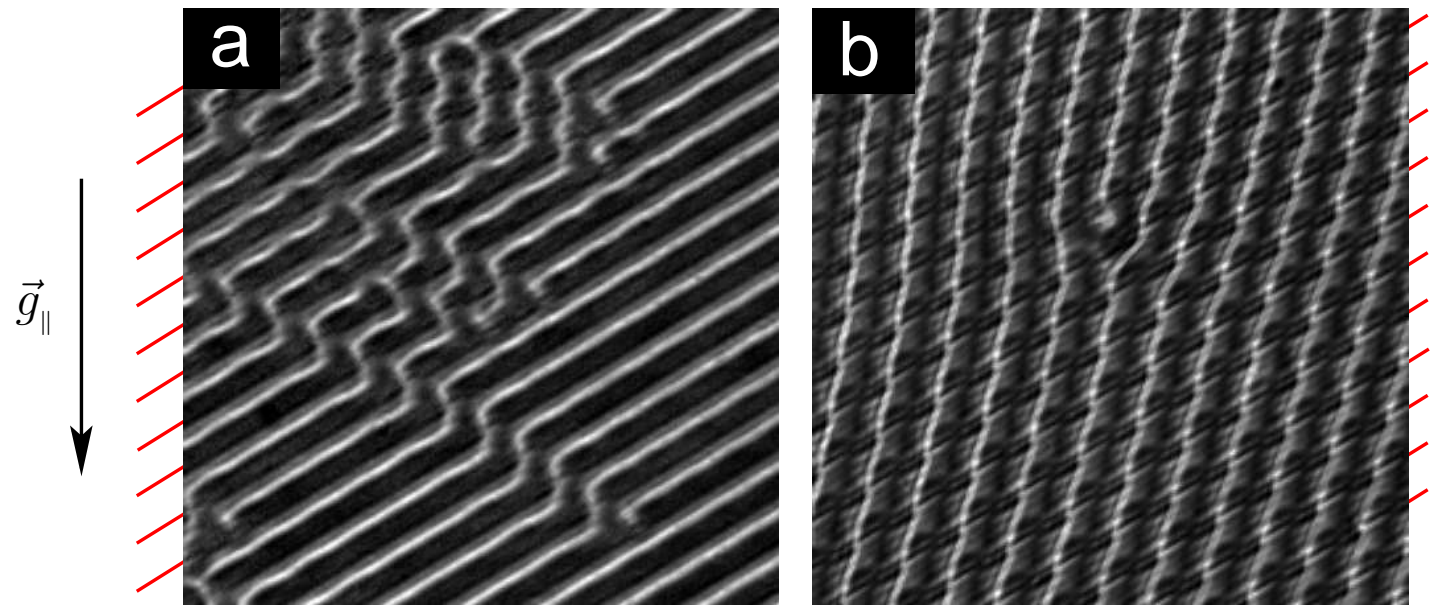

Figure 4.44: Kinks (a) at $\gamma=10^{\circ}$ and $\varepsilon=0.35$ and oblique longitudinal rolls (b) at $\gamma=10^{\circ}$ and $\varepsilon=2.57$. The red stripes mark the forcing texture.

A new pattern occurs for inclination angles $10^{\circ}<\gamma<53^{\circ}$. Here, forced oblique rolls become unstable to an oblique bimodal pattern (OB). In addition to the forcing wave vector $\vec{q}_{f}$ a second mode $\vec{q}_{1}$ occurs, which strongly depends on $\gamma$ (Fig. 4.45). The modulus of $\vec{q}_{1}$ and its angle with respect to $\vec{q}_{f}$ ( $\alpha$ in Fig. 4.45) grows from $\alpha \approx 70^{\circ},\left|\overrightarrow{q_{1}}\right| \approx 0.8 q_{f}$ at $\gamma=15^{\circ}$ to $\alpha \approx 120^{\circ}$ and $\left|\vec{q}_{1}\right| \approx 1.1 q_{f}$ at $\gamma=85^{\circ}$, where it saturates (see Fig. 4.46). Thus the structure changes qualitatively at approximately $\gamma=53^{\circ}$ from a rhombic symmetry to oblique hexarolls (OX).

Especially in the range $\gamma=60^{\circ}-75^{\circ}, \vec{q}_{1}$ (red) has the same length as $\vec{q}_{f}$ and is with an angle of $\alpha=120^{\circ}$ aligned to it. As seen in Fig. 4.45 one can identify a hexagonal structure, where a former higher order mode of the rhombic structure turns to provide a third wave vector $\vec{q}_{2}$ with the same length as $\vec{q}_{f}$ and $\vec{q}_{1}$ and directed $120^{\circ}$ to both. Although it is a continuous transition from rhombic to oblique hexarolls a corresponding transition line can be set at an inclination angle $\gamma=48^{\circ}$. According to this subjective choice rhombic and oblique hexarolls were distinguished in Fig. 4.43. 

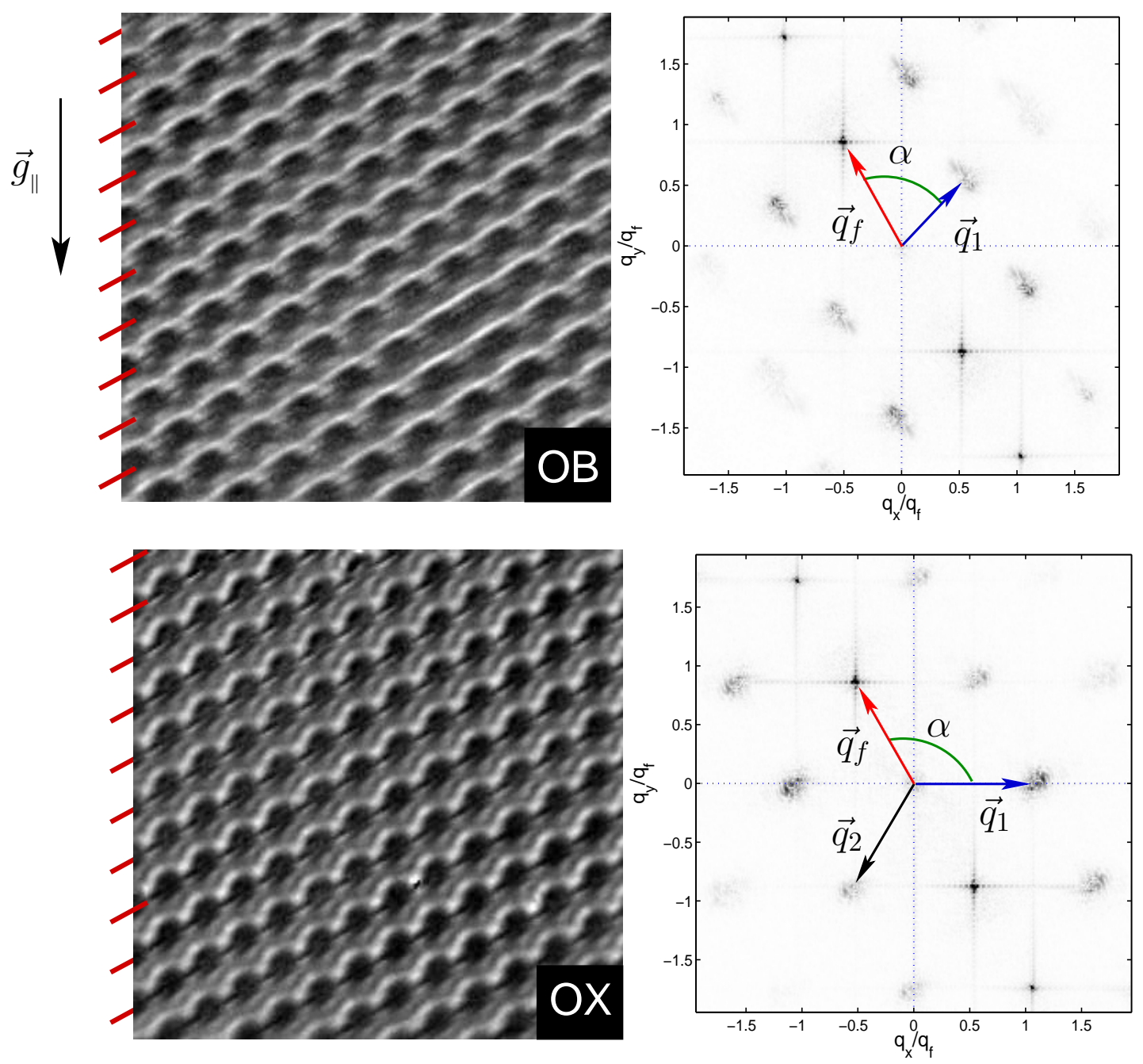

Figure 4.45: Rhombic pattern at $\gamma=20^{\circ}$ and $\varepsilon=0.146$ (top) and oblique hexarolls at $\gamma=70^{\circ}$ and $\varepsilon=0.08$ (bottom) and their corresponding Fourier transform. Red stripes mark the forcing texture at the bottom plate. 


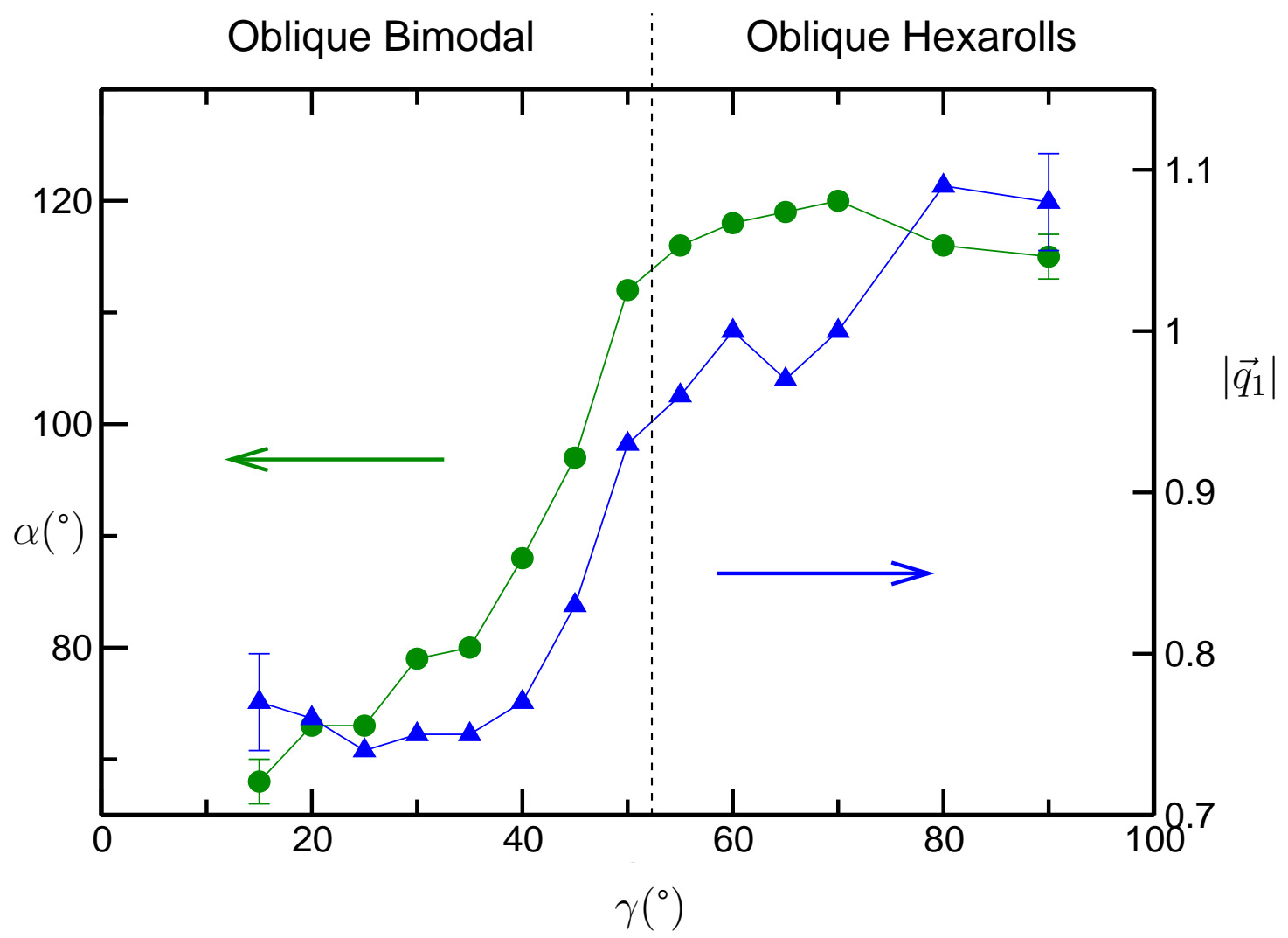

Figure 4.46: Transition from oblique bimodal to oblique hexarolls. The plot shows the development of mode $\vec{q}_{1}$ in dependence of inclination angle $\gamma$. The brown line at around $\gamma=52^{\circ}$ marks the transition from rhombic pattern to oblique hexarolls.

Rhombic and oblique hexarolls are drifting slowly along the SU-8 ridges in downhill direction. In addition, phase and amplitude defects occur with increasing $\varepsilon$ and the pattern becomes more dynamic. When $\varepsilon$ is increased the large scale shear flow is increased as well and becomes more and more dominant over the forcing. The rolls are still phase locked with the underlying SU-8 ridges. If the modulation strength gets large enough, localized vertical connections are created between adjacent rolls. A staircase like pattern occurs, similar to coherent kinks. With increasing $\varepsilon$ one sees more such patterns resulting in a mobile structure of crawling rolls (Fig. 4.47).

While for small inclination angles here the crawling rolls are more like longitudinal rolls with several dynamical "crawling" defects, at higher inclination angles the number of defects increases strongly and the pattern becomes spatio-temporal chaotic with a loss of coherence. Although the morphology of crawling rolls differs significantly from small inclination angles to large angles, a further categorization in different kinds of crawling rolls seems not to be useful. 

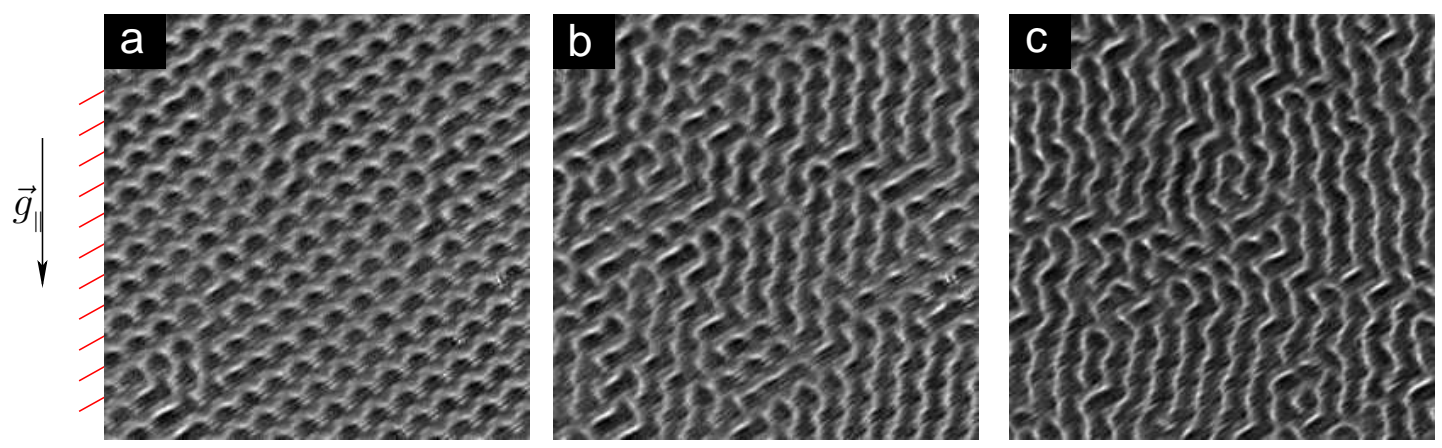

Figure 4.47: Development of $\mathrm{CR}$ at $\gamma=20^{\circ}$. (a) Oblique bimodal pattern with localized defects at $\varepsilon=1.21$. (b) Not yet fully developed crawling rolls as $\varepsilon=1.25$. (c) Fully developed crawling rolls at $\varepsilon=1.4$. The red stripes mark the forcing texture on the bottom plate.
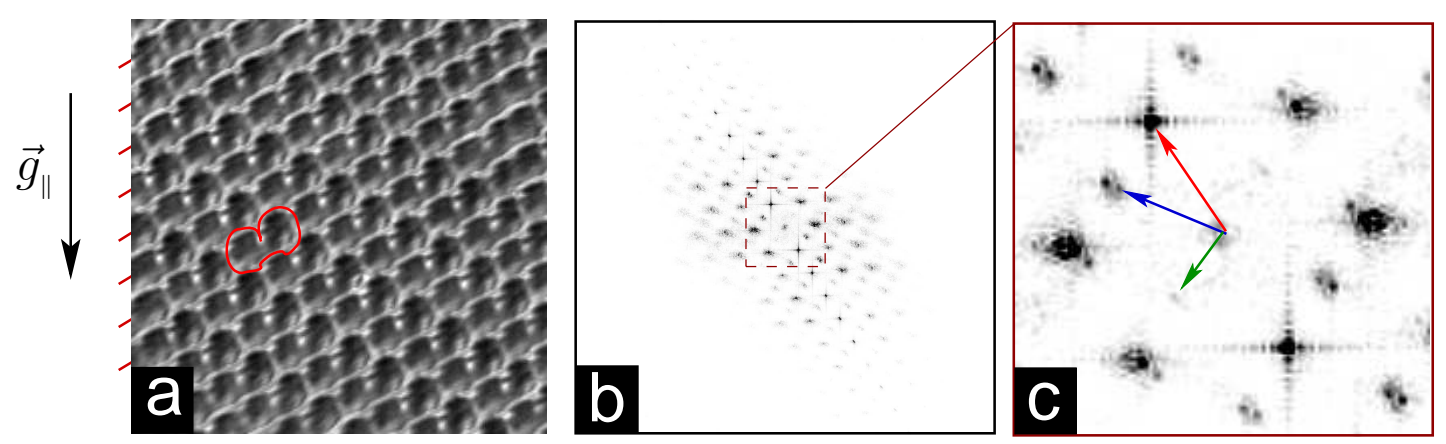

Figure 4.48: Pretzel pattern (a), its Fourier transform (b) and the center of the Fourier spectrum (c) (taken at $\gamma=90^{\circ} \varepsilon=0.06$. Red stripes mark the forcing structure.

For high inclination angles $\gamma>75^{\circ}$ again a superlattice lattice occur as an instability of oblique hexarolls for higher values of $\varepsilon$. Here, a pretzel (PZ) like structure sits on a rhombic lattice. The corresponding reciprocal vectors are: $\vec{q}_{3}=0.5 \vec{q}_{2}$ (green arrow in Fig. 4.48b) and $\vec{q}_{4}=0.5\left(\vec{q}_{f}-\vec{q}_{1}\right)$ (blue arrow in Fig. 4.48) as shown in the Fourier transform. The basic vector $\vec{q}_{4}$ is strongly suppressed by a local minimum in the Fourier spectra of a single pretzel and therefore only higher modes are visible. Increasing $\varepsilon$ to even higher values leads again to phase turbulent crawling rolls (CR). 


\section{Chapter 5}

\section{Forcing with Various Wave Numbers}

In this chapter the effect of one dimensional forcing on conventional RayleighBénard convection without inclination is investigated. The forcing wave number $q_{f}$ is changed and the occurring patterns are shown in a $q_{f^{-}}$phase diagram. These experiments are the direct continuation of work done by John McCoy at Cornell University [68].

Since it is costly and time consuming to buy a bottom plate and print a texture via photo lithography on its surface, only one such bottom plate was used for all experiments. In this way the forcing pattern was constant throughout all experiments. But since all parameter are in units of the cell height $d$, one can change the cell height in order to change the (dimensionless) forcing wave number. This was done for the experiments presented here. Unfortunately, by changing the forcing wave number in this way, one automatically changes the forcing strength $\delta$ which is a direct function of the ratio between the height of the SU-8 stripes $(\approx 65 \mu \mathrm{m}$, see previous chapter $)$ and the cell height $d$. In particular, for small forcing wave numbers $\left(q_{f}=0.7 q_{c}\right)$, the height of the structure reaches $15 \%$ of the whole cell height and one can not consider this boundary forcing anymore as a small perturbation.

Experiments at large wave numbers $\left(q_{f} / q_{c}>1\right)$ were conducted at an average temperature $T_{a v}=25^{\circ} \mathrm{C}$ and a pressure $p \cong 48 \mathrm{bar}(700 \mathrm{psi})$. These two parameters result in an effective Prandtl number of $\operatorname{Pr}=1.27$. For experiments at lower $q_{f} / q_{c}$ the cell height was lowered and therefore the critical temperature difference increased significantly which led to experimental problems. Therefore, in order to reach high Rayleigh numbers, the average temperature was increased to $T_{a v}=27^{\circ} \mathrm{C}$ and the pressure to $p=50.0 \mathrm{bar}(725 \mathrm{psi})$, resulting in a Prandtl number of $\operatorname{Pr}=1.30$.

The instability of forced rolls was also investigated numerically [97]. Regarding 


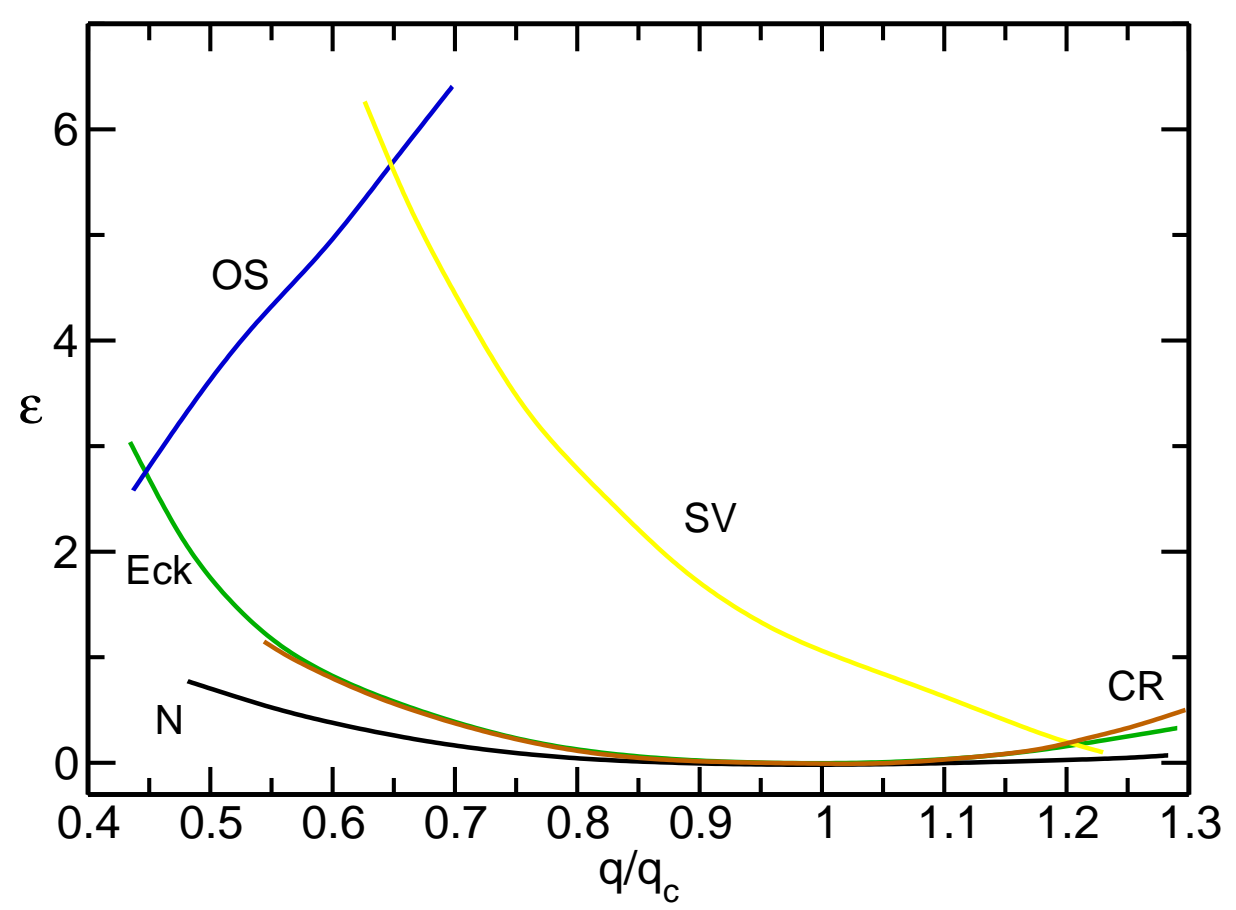

Figure 5.1: Busse balloon for $\operatorname{Pr}=1.27$. The region for stable straight rolls is confined by skewed varicose instability (SV), oscillatory instability (OS), cross roll instability (CR), and the Eckhaus instability (ECK). The solid black line marks the neutral curve $(\mathrm{N})$.

these calculations, the change in $\operatorname{Pr}$ of \pm 0.03 can be neglected. As calculated for $q_{f}=1.05 q_{c}$, the instability of straight rolls would grow from $\varepsilon=1.05$ at $\operatorname{Pr}=1.27$ to $\varepsilon=1.06$ at $\operatorname{Pr}=1.30$. This is less then the size of the symbols in Fig. 5.15.

The Busse balloon for $\operatorname{Pr}=1.27$ is shown in Fig. 5.1. ${ }^{1}$ At higher control parameter $\varepsilon$, straight rolls become unstable with respect to an Oscillatory (OS) or a skewed-varicose (SV) instability. At lower $\varepsilon$ the region of stable straight rolls is confined by an Eckhaus instability (ECK) and a cross roll instability (CR). Note, that the brown line for CR and the green line for ECK lie on top of each other for $q / q_{c}<1$, whereas for larger $q$ CR lies above ECK.

One expects now, that in the forced case rolls with wave number inside the Busse

\footnotetext{
${ }^{1}$ Thanks again to Prof. W. Pesch who provided the appropriate code to check stability of straight rolls with various wavelength
} 
balloon should be stable as well, whereas forcing a wave number outside will lead to new interesting patterns. The system will choose a configuration where it can develop modes inside the balloon which resonate together to the forcing mode. Several of the phenomena that occur for $q_{f} \approx q_{c}$ were already discovered in previous experiments by J. McCoy (see [68, 69]), but are explained and described here in more detail.

As pointed out in previous chapters, spatial periodic forcing leads to convection, even for negative control parameter $\varepsilon$. Interesting now is the behavior of the system if the these rolls become unstable, if e.g. the forcing wave vector $q_{f}$ lies outside the Busse balloon for unforced convection. These instabilities are listed and described in the following sections.

\subsection{Varicose Pattern}

For forcing with wave numbers in the range of $1.00 q_{c}<q_{f}<1.13 q_{c}$, forced rolls become first unstable to a varicose pattern as already discussed in Chapter 4 and shown in Fig. 5.2.
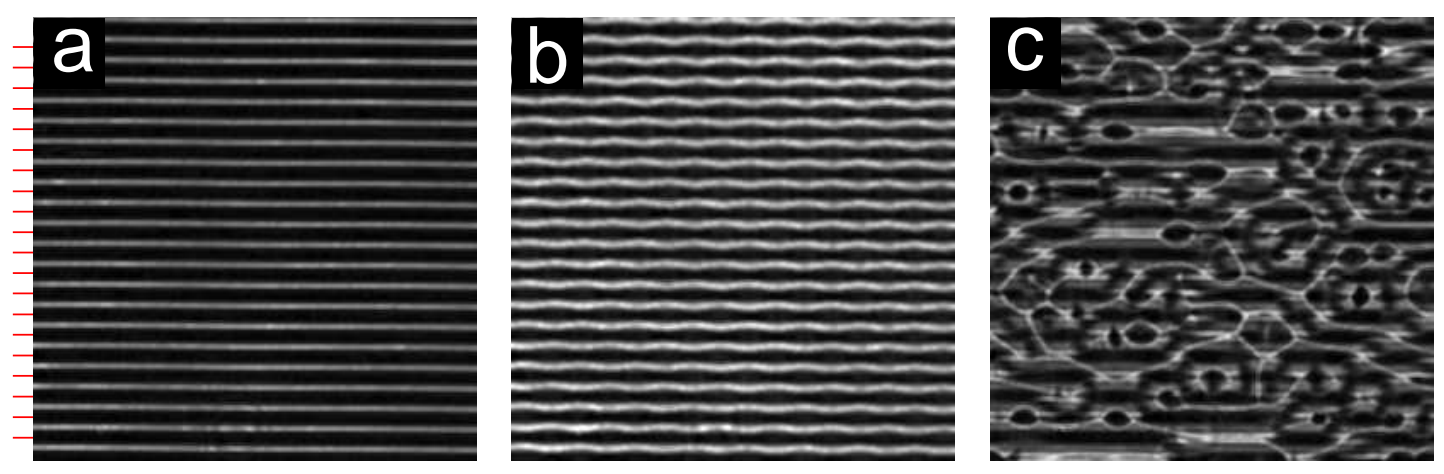

Figure 5.2: Forced Straight rolls at $\varepsilon=2.13$ (a). Varicose Pattern at $\varepsilon=2.76$ (b). Spatio-temporal chaos at $\varepsilon=2.79$ (c). Experiment conducted at $q_{f}=1.08 q_{c}$. Red stripes mark the bottom plate structure.

With respect to Fig. 5.2, varicose pattern consists of the forcing wave vector $\vec{q}_{f}=q_{f}(0,1)$ and two symmetric modes which modulate the phase:

$$
\vec{q}_{1}=\left(\begin{array}{c}
q_{x} \\
0.5
\end{array}\right) \quad \text { and } \quad \vec{q}_{2}=\left(\begin{array}{c}
-q_{x} \\
0.5
\end{array}\right)
$$

In fact, the value of $q_{x}$ shows a slight dependency on the forcing wave number $q_{f}$. As shown in Fig. 5.3, the value of $q_{x}$ increases with increasing forcing wave number $q_{f}$. Note, that this increase is smaller than the error bars and only 


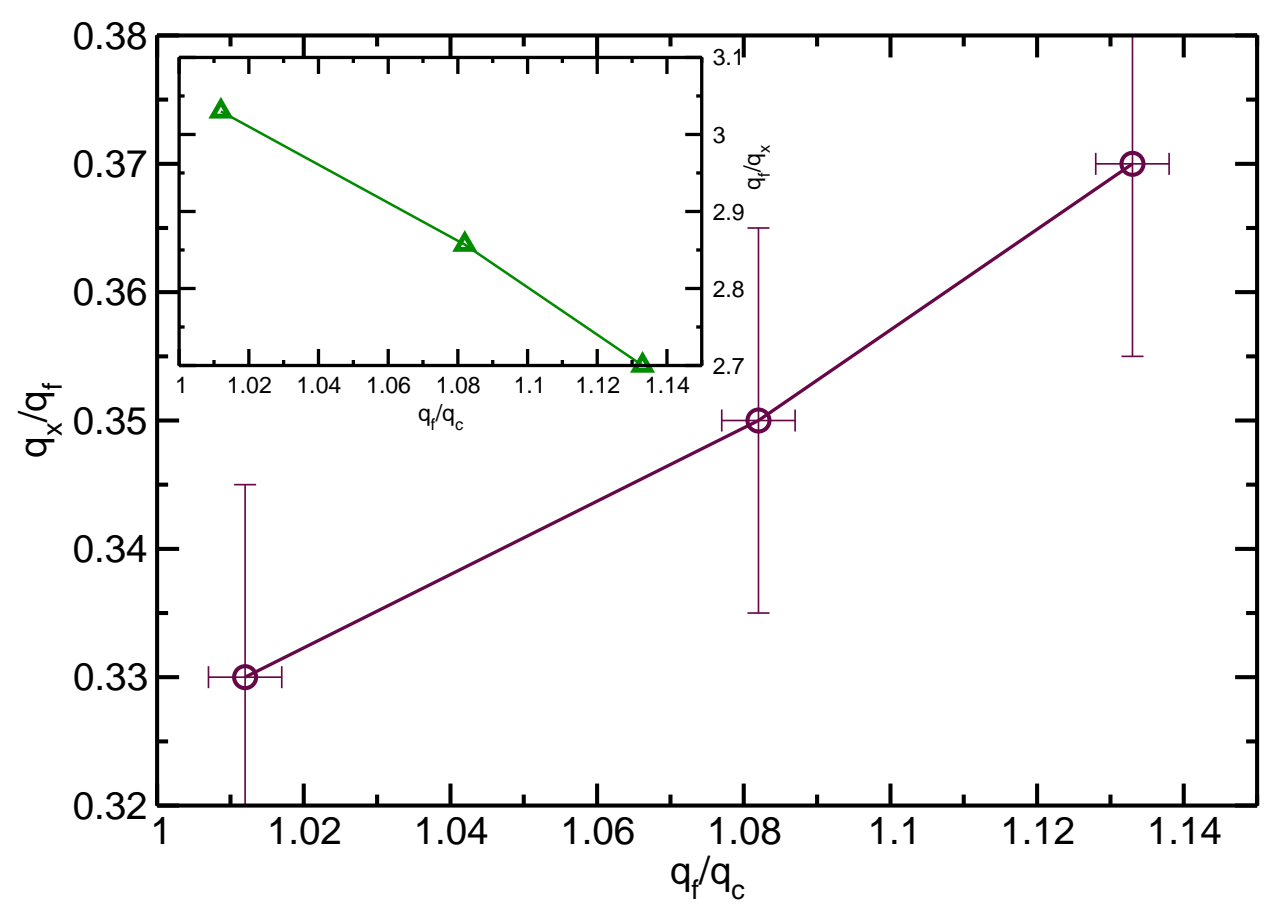

Figure 5.3: Development of the varicose mode $q_{x}$ as a function of $q_{f}(\circ)$. In the inlet the corresponding wavelength $1 / q_{x}$ is plotted $(\triangle$.)

three points are shown, and thus the correlation between $q_{x}$ and $q_{f}$ might be less significant than it seems to be on the first glance. On the other hand, also the corresponding wavelength $2 \pi /\left(q_{x} \lambda_{f}\right)=q_{f} / q_{x}$ is shown in Fig. $5.3(\triangle)$. This is the length of the phase modulation in $\mathrm{x}$-direction. The decrease here agrees nicely with a decrease of the length of coherent kinks for higher forcing wave numbers as will be explained in the next section (see Fig. 5.8).

For higher control parameter $\varepsilon^{2}$ the phase of the rolls is so strongly modulated, that adjacent rolls merge and the system turns immediately into a spatiotemporal chaotic state as shown in Fig. 5.2c.

The amplitude development for the forcing mode $\vec{q}_{f}$ and for the side mode $\vec{q}_{1}$ is shown in Fig. 5.4. Note, the amplitude of the corresponding power spectrum $|F(\vec{q})|^{2}$ were calculated. The left plot shows the amplitude of both modes. The

\footnotetext{
${ }^{2}$ The exact value for which VP become unstable and spatio-temporal chaos occurs depends on the forcing wave number. For $q_{f}=1.08 q_{c}$ for example, VP turn into spatio-temporal chaos at $\varepsilon=2.79$.
} 


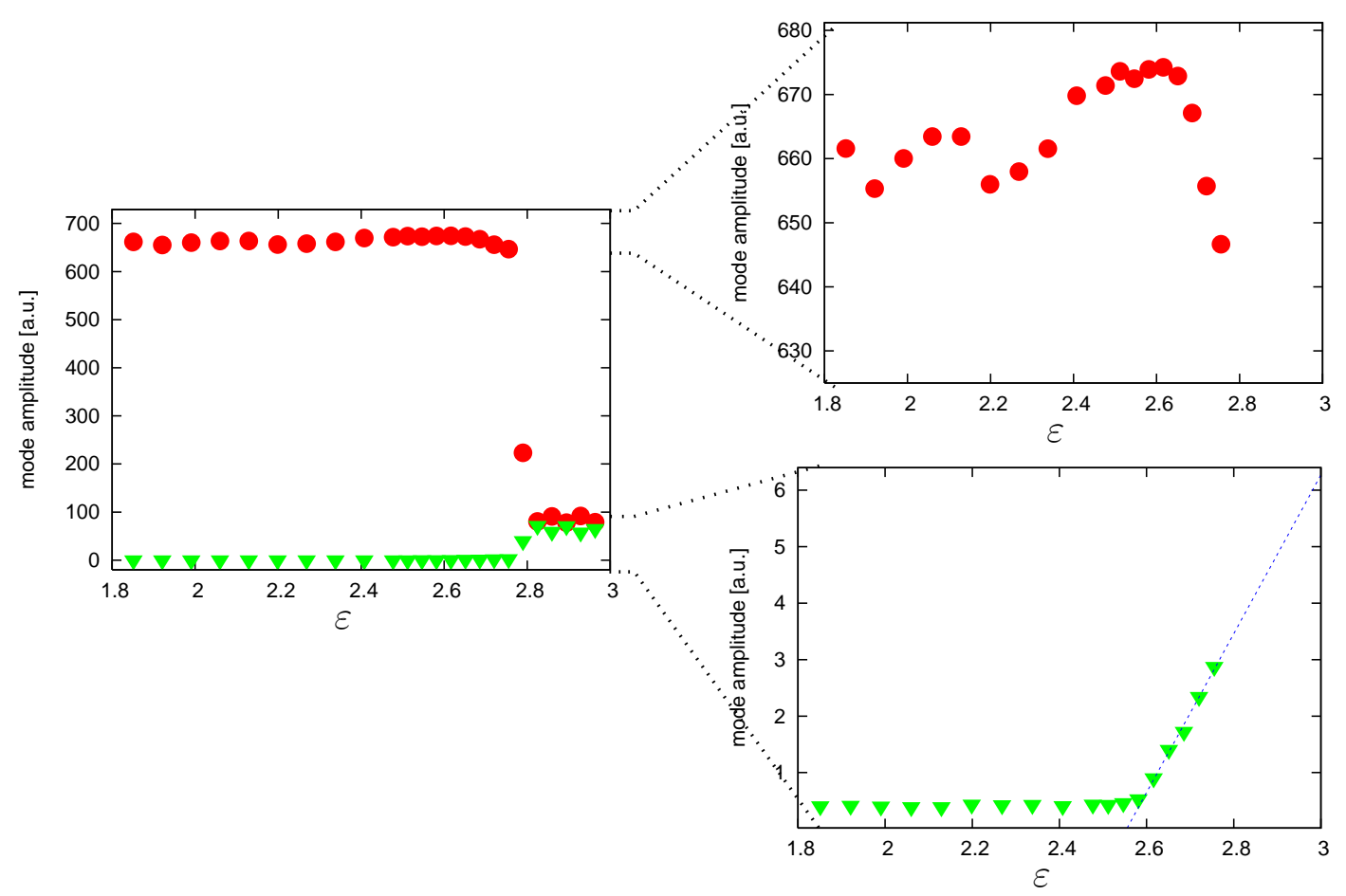

Figure 5.4: Amplitude evolution of the forced mode $\vec{q}_{f}(\bullet)$ and of $\vec{q}_{1}(\nabla)$ in the power spectrum. The left plot shows the complete evolution of both amplitudes. The right plots show enhanced sections. A linear curve was fitted to the amplitude of $\vec{q}_{1}$ at onset of VP as a guide to they eye.

lower green curve (amplitude of $\vec{q}_{1}$ ) is initially zero and start to increase slightly at $\varepsilon=2.6$, when varicose pattern set in. The bifurcation is a forward bifurcation as can be seen in the lower right plot, where the y-axis is expanded. The amplitude of the forcing mode $\vec{q}_{f}(\bullet)$ decreases at the same time. Unfortunately, for these experiments the onset of varicose appeared already at very high temperature differences, where the shadowgraph setting is not in the linear regime anymore. Therefore, the amplitude of $\vec{q}_{f}$ shows a more irregular behavior before the onset of VP, where a smooth increase was expected. For the amplitude of $\vec{q}_{1}$ the shadowgraph setting is not so important, since the phase is modulated, which can easily be detected even with a shadowgraph setting close to the first caustic.

The onset of the chaotic pattern results in a drop of amplitude of $\vec{q}_{f}$ and a strong increase of the amplitude of $\vec{q}_{1}$ at $\varepsilon=2.79$.

The varicose pattern was also observed by McCoy [68, 69], but only as a transient state. By increasing $\Delta T$ fast VP occurred, but became immediately unstable to spatio-temporal chaos. For a slow adiabatic increase of $\Delta T$, he observed kink structures with an interesting dynamical behavior such as clustering of kinks 
of the same kind and annihilation of opposite kinks. His experimental results differ from the one in this work, since he used $\mathrm{SF}_{6}$ as a working fluid and thus, the Prandtl number was $\operatorname{Pr}=0.9$ instead of 1.3 as here. The second and more important difference is, that he used a forcing wave number of $q_{f}=6 / 5 q_{c}$ which is larger than the largest forcing wave number $\left(q_{f}=1.13 q_{c}\right)$ for which non-transient varicose pattern were observed. For lower values of $q_{f}$, kinks were also found in the presented experiments. These kinks are object of the next section (see also the phase diagram in Fig. 5.15).

Similar to McCoy's case, a hysteretic behavior was observed. Varicose patterns could only be observed when the temperature difference was increased. A decrease in $\varepsilon$ from the spatial temporal chaotic state only decreases the disorder but does not turn the system back to VP. Instead, a regime exist where localized kinks are observed, which behave in the same way as reported by McCoy [68]. The kinks move around, annihilate when meeting negative phase kinks and cluster with kinks of the same kind. Kink clusters are much less mobile as single kinks - in fact they are not mobile at all.

\subsection{Coherent Kinks}

As one increases the forcing wave number by increasing the cell height $d$, for $q_{f}>1.2 q_{c}$ various kink lines occur as an instability of forced straight rolls. They are similar to the kinks reported already in the previous chapter (Sec. 4.2.4). The dynamics of such kinks was reported and described in detail for $q_{f}=\frac{6}{5} q_{c}$ by McCoy $[68,69]$. In general, these kinks are $2 \pi$-phase jumps which are aligned obliquely with respect to the forcing ridges. If two kinks come close to each other, they either annihilate or cluster together in dependency on whether the phase jump is positive or negative.

In agreement to McCoy's observations, kinks align always along a straight line, at their first occurrence. A false color shadowgraph image of a kink line (at $\left.q_{f} / q_{c}=1.19, \varepsilon=1.76\right)$ is shown in Fig. 5.5a. One can decompose the image and calculate the phase of the in y-direction periodically alternating pattern as described in Chapter 3. The phase field of the shadowgraph image of Fig. 5.5a is shown in Fig. 5.5(b and c). In Fig. 5.5a, the lines of cold down flow become very thick at the kink. This is a shadowgraph effect of the underlying SU-8 structure, which becomes visible if the rolls are not parallel to the structure but build an angle with it.

A single kink line in an otherwise parallel roll pattern can be given by the formula:

$$
\psi(x, y)=A \cos \left(\vec{q}_{f} \vec{r}+\pi \cdot \operatorname{erf}(x / a+y / b+c)\right)
$$



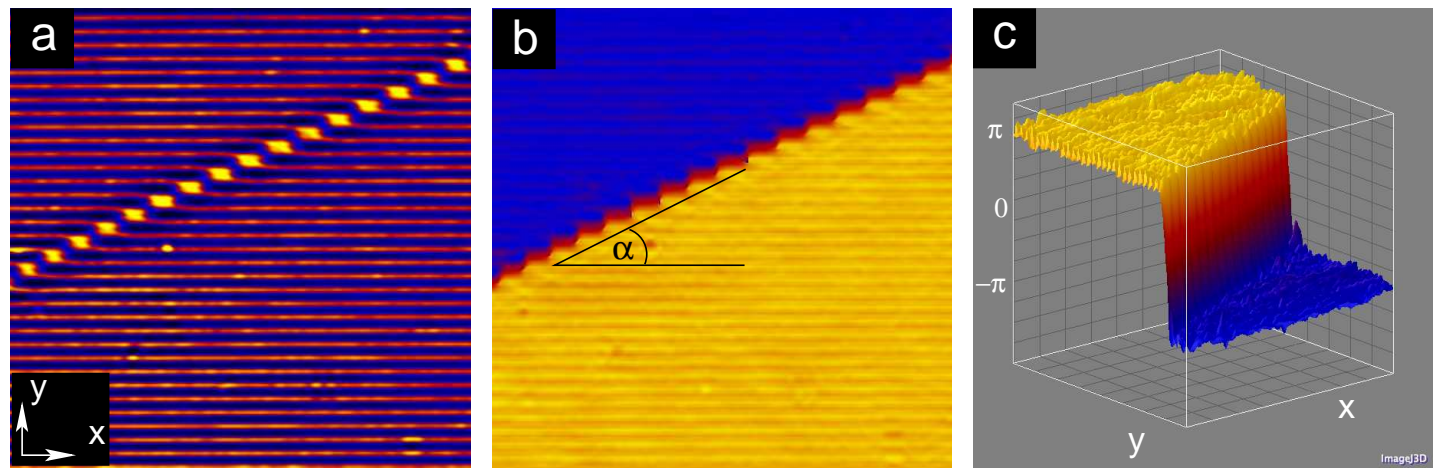

Figure 5.5: False color image of kinks (a), their corresponding phase plot as image (b) and surface plot (c) and a calculated image regarding formula 5.1 (d)

The phase alone is written by an error function. The parameter $a$ and $b$ are typical length scales for the phase step in $\mathrm{x}$ - and in y-direction and $c$ is a shift of the whole kink line. In fact, within $-a<x<a$ (or $-b<y<b$ ) the phase changes from $-0.85 \pi$ to $0.85 \pi$. Thus, the real step width corresponds rather to $2 a$ and $2 b$. Kink lines often drift slowly in one direction with a constant velocity and hence $c$ is a linear function of time. A very important quantity is the angle of the kink line, denoted $\alpha$ in Fig. 5.5b. This angle is given by:

$$
\alpha=\operatorname{atan}(b / a)
$$

and can easily be measured in the experiment. The sign of $b$ has to be negative in order to produce kinks. If $b$ is positive one sees a structure which is similar to the skewed varicose instability observed in non-forced Rayleigh-Bénard convection with Prandlt numbers close to one (see Fig. 5.6). One should note here, that close to its onset for $\operatorname{Pr} \approx 1$ the skewed varicose consist of an instability wave vector with modulus $q_{i} \approx 0$, meaning the corresponding wavelength is larger than the cell size. Only for larger $\varepsilon$ the wavelength of the fastest crowing mode becomes small enough to be observed as a periodic modulation of the convection rolls as presented by [34]. A step function as given by the error-function can be seen as a good approximation for the occurring jump in phase.

Figure 5.7 shows kink lines for three different $q_{f}$. One sees clearly, that the angle of the kink line $(\alpha)$ increases with increasing $q_{f}$. In addition, the parameters $a$, and $b$ which determines the characteristic length scale under which a phase jump occurs are drastically reduced. While for $q_{f} / q_{c}=1.191$ (Fig. 5.7a) the kink is shaped like a "S", for higher $q_{f}$ kinks look more like a rectangular step function (Fig. 5.7b).

In Fig. 5.8, the step width in y-direction $2 b(\boldsymbol{\Delta})$ is plotted for four different values of $q_{f}$. The value of $2 b$ decreases monotonically with increasing $q_{f}$. As we 

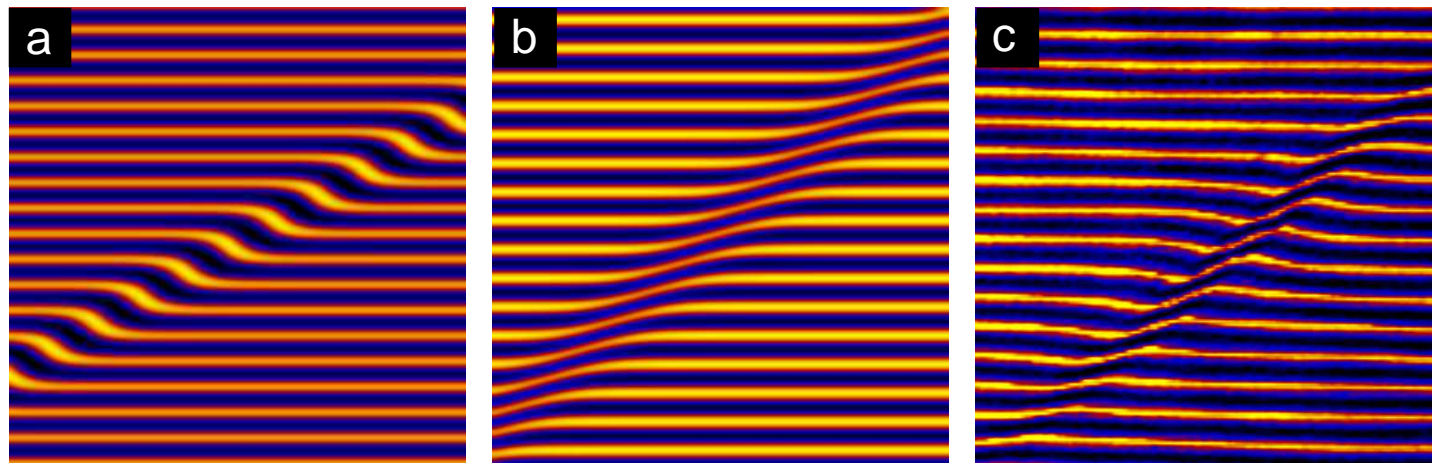

Figure 5.6: Comparison between kinks and skewed varicose. A kink like structure given by formula 5.1 with $a=2 \lambda_{f}$ and $b=-\lambda_{f}$ (a) and the same formula plotted with $a=b=2 \cdot \lambda_{f}(\mathrm{~b})$. An experimentally observed skewed varicose instability is shown in (c) (taken from [75]).
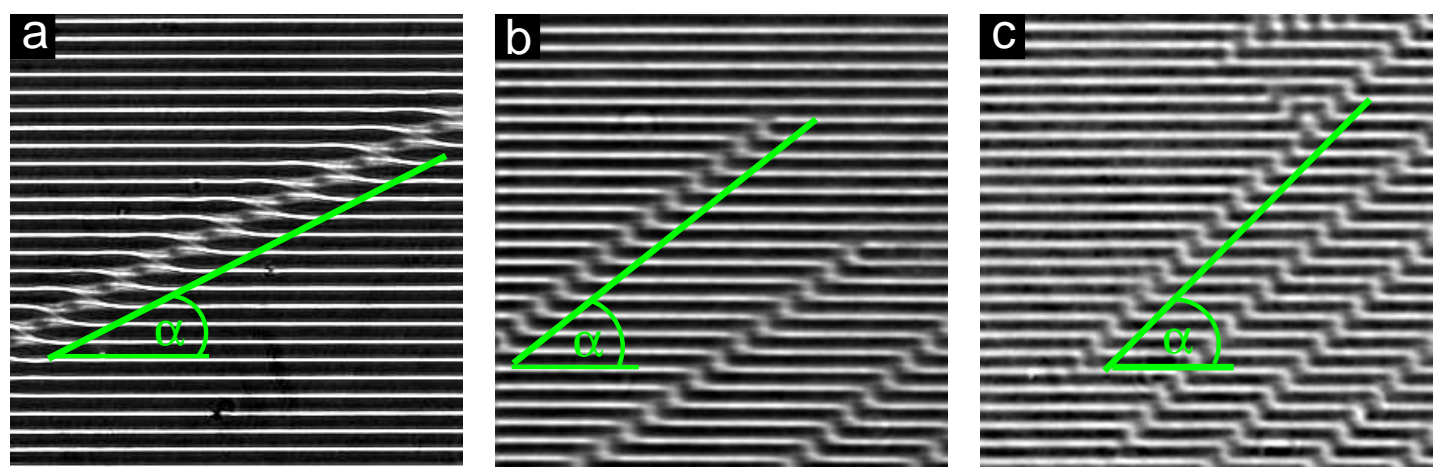

Figure 5.7: Kink lines for different forcing wave numbers: (a): $q_{f} / q_{c}=1.191$; (b): $q_{f} / q_{c}=1.276 ;(\mathrm{c}): q_{f} / q_{c}=1.321$ 
will see later on (Sec. 5.5) the instability line of forced straight rolls decreases continuously as a function of $q_{f}$ in the range where straight rolls turn to kinks and VP (Fig. 5.15). Neither this instability line, nor its slope shows any indication of a phase transition. Furthermore in preliminary simulations [97], only one instability was found where in the experiments instabilities to VP and kinks were observed. Therefore one might speculate that kinks and VP are two different expressions of the same instability.

For comparison we need to find similar quantities. One can compare the step width in $\mathrm{x}$-direction of the kinks $(2 a)$ with the modulation wavelength in $\mathrm{x}$ direction $\lambda_{x}$ of the VP (see (c) and (d) in Fig. 5.9). This $\lambda_{x}$ is nothing else then $\lambda_{x}=2 \pi / q_{x}$, with $q_{x}$ being the x-component of the two side modes $\left(\hat{x} \cdot \vec{q}_{1,2}\right)$ of the varicose pattern (see previous section). Within both length scales ( $2 a$ and $\lambda_{x}$ ) the phases of the corresponding pattern changes in x-direction by (nearly) $2 \pi$.

The step width of the kinks in y-direction $2 b$ can then be compared in an analog way with the wavelength corresponding to the y-component of the side modes of the varicose pattern $\lambda_{y}=2 \pi /\left(\hat{y} \cdot \vec{q}_{1,2}\right)=2 \lambda_{f}$. A $\triangle$ is plotted therefore as well in the diagram at $2 b=2 \lambda_{f}$ to represent the corresponding value for VP. Apparently this point fits well with the monotonic decrease of the step width of the kinks $(2 b)$.

Now it might also be interesting to compare the step width in x-direction $2 a$ of the kinks with the modulation length in $\mathrm{x}$-direction $\lambda_{x}$ of the VP. This is done in Fig. 5.9a. The step width of the kinks $(2 a)$ decreases monotonically with $q_{f}(\boldsymbol{\Delta})$. A similar decrease with increasing $q_{f}$ can also be observed for $\lambda_{x}$ of the varicose pattern $(\triangle)$. Note that the same data were already plotted in the inlet of Fig. 5.3. However, there is a discontinuity between the data for VP and that for the kinks. If one plots instead the doubled wavelength for the varicose pattern $\left(2 \lambda_{x}\right)$, the transition becomes more continuously $(\triangle)$.

That appears on the first glance as a cheap trick, since it exists of course always a factor that shifts the data in the right way. But here, there might be some systematic in it since also for the comparison of the angles a length in x-direction of $2 \lambda_{x}$ instead of $\lambda_{x}$ leads to a more continuous transition between VP and kinks. This can be seen in Fig. 5.9b. As mentioned already above, the angle of the kink lines $\alpha(\bullet)$ increases monotonic with increasing $q_{f}$. Now one would like to compare also this quantity with a similar quantity of the varicose pattern. If one compares $\lambda_{y}$ of the $\mathrm{VP}$ with $2 b$ of the kinks and $\lambda_{x}$ with $2 a$, than of course one can compare the angle of the kink line $\alpha=\operatorname{atan}(b / a)$ with the following angle of the VP: $\beta=\operatorname{atan}\left(\lambda_{y} / \lambda_{x}\right)$. This angle is nothing else than the angle between the side mode $\left(\vec{q}_{1,2}\right)$ and the y-axis in the Fourier space (see Fig. $5.8 \mathrm{~d}$ ). The angle $\beta$ is plotted with $\circ$. Even though also $\beta$ shows a monotonic increase, it does not fit with the data of $\alpha$ for the kinks. However, if one instead plot another quantity $\chi=\operatorname{atan}\left(\lambda_{y} /\left(2 \lambda_{x}\right)\right)(\circ)$. This angle of VP fits now well in the monotonic behavior 


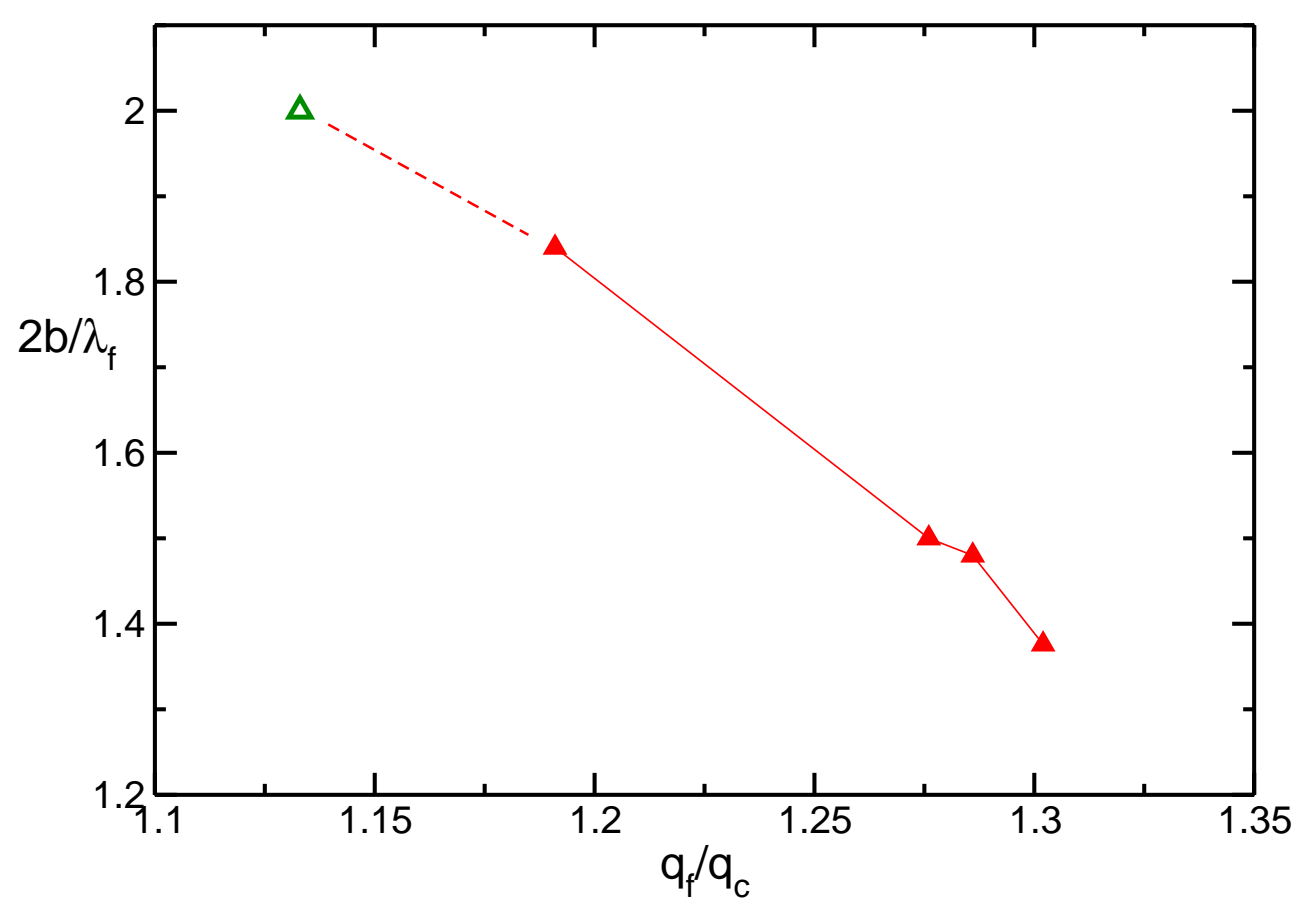

Figure 5.8: Step width in y-direction of the phase of a kink line $(2 b, \mathbf{\Delta})$ as a function of the forcing wave number $q_{f} / q_{c}$. In addition it is shown the wavelength in y-direction of the side mode of the varicose pattern: $\lambda_{y}=2 \pi\left(\hat{y} \vec{q}_{2}\right)=2 \pi / q_{y}=2 \lambda_{f}(\triangle)$.

of the angle $\alpha$ for the kink lines.

In fact we don't know in detail which quantity of VP one needs to compare with which quantity of kinks and it is also not clear where the factor 2 comes from. Nevertheless, there are some hints, that varicose pattern and kinks might be produced by one and the same instability. If angle and wavelength of this instability are in resonance with the forcing wave number, VP occur, otherwise kinks will be created.

\subsection{Cross Rolls}

At $q_{f}>1.32 q_{c}$ forced rolls become unstable at $\varepsilon \approx 0$. A cross roll like instability with a new mode $\vec{q}_{1}$ occurs, perpendicular to the forced mode $\vec{q}_{f}$ (Fig. 5.10): 

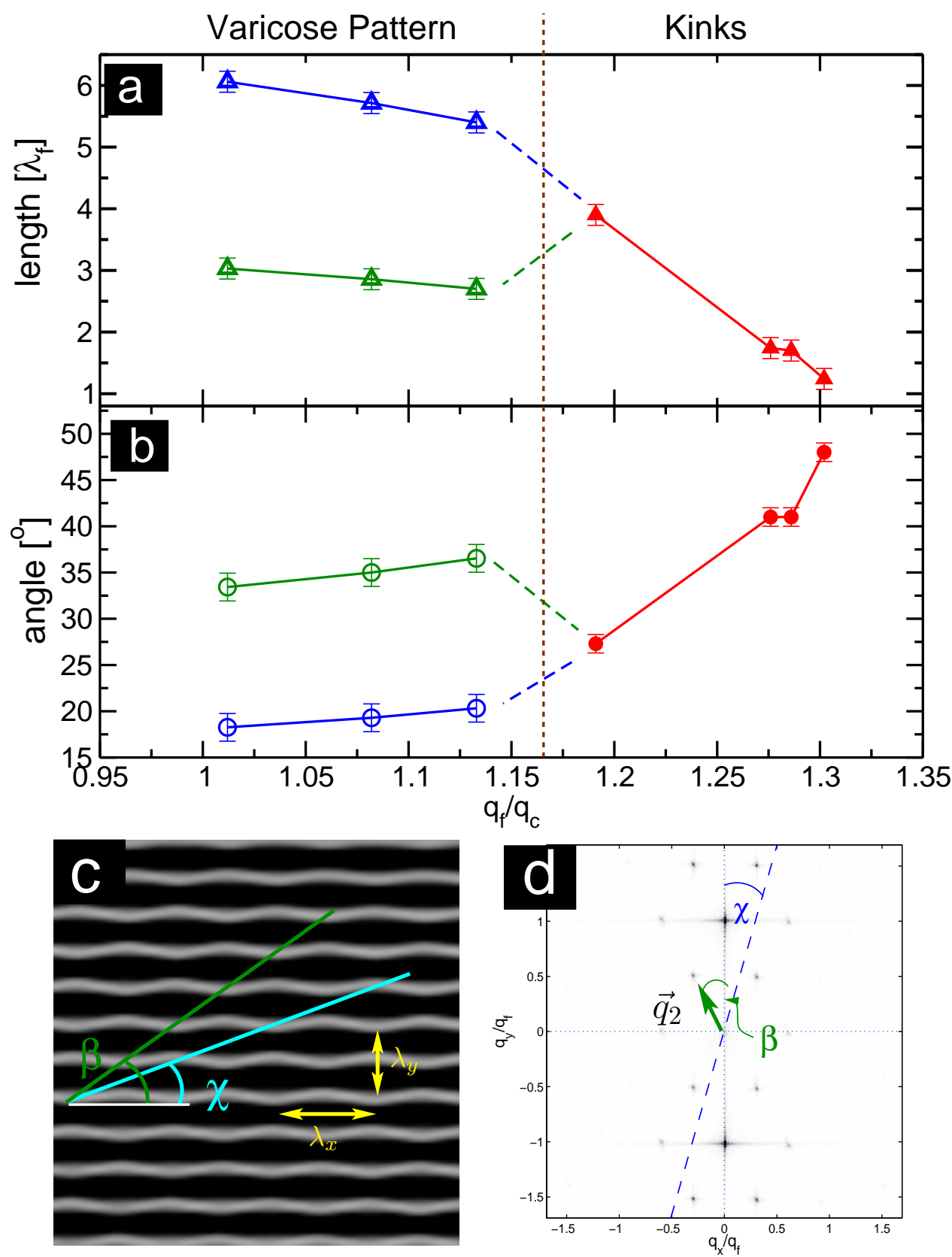

Figure 5.9: (a): Comparison between step width in x-direction of the kinks $(2 a$, $\Delta)$ and the corresponding value for the $\operatorname{VP}\left(\lambda_{x}, \Delta\right)$. In addition, the doubled wave length is plotted $\left(2 \lambda_{x}, \Delta\right)$. (b): Development of the angle of the kink line $(\alpha, \bullet)$ and the angle of the side mode of VP with the y-axis $\left(\alpha=\operatorname{atan}\left(2 \lambda_{f} / \lambda_{x}\right), \circ\right)$ as a function of the forcing wave number $q_{f}$. In addition, another angle is plotted that corresponds to $\beta=\operatorname{atan}\left(2 \lambda_{f} /\left(2 \lambda_{x}\right)\right)$ (o). (c) and (d) shows the VP and a corresponding Fourier transform and marks the angles $\alpha$ and $\beta$ as well as the modulation length in $\mathrm{x}$ - and y-direction $\left(\lambda_{x}\right.$ and $\left.\lambda_{y}\right)$. 


$$
\vec{q}_{1}=\left(\begin{array}{c}
q_{c} \\
0
\end{array}\right)
$$

(a)

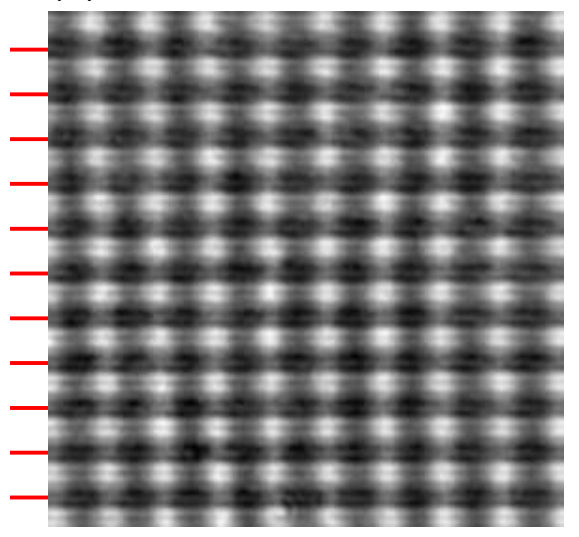

(b)

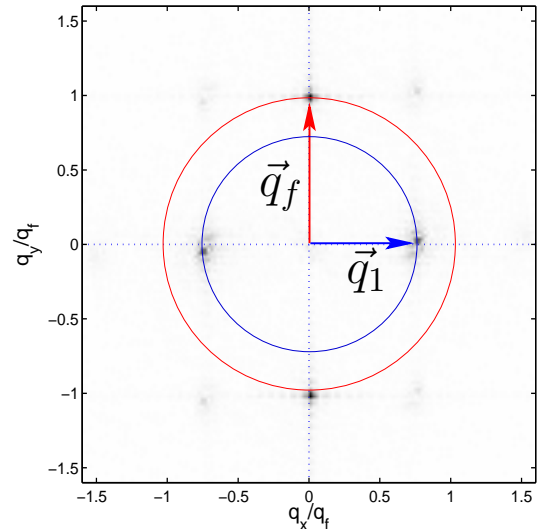

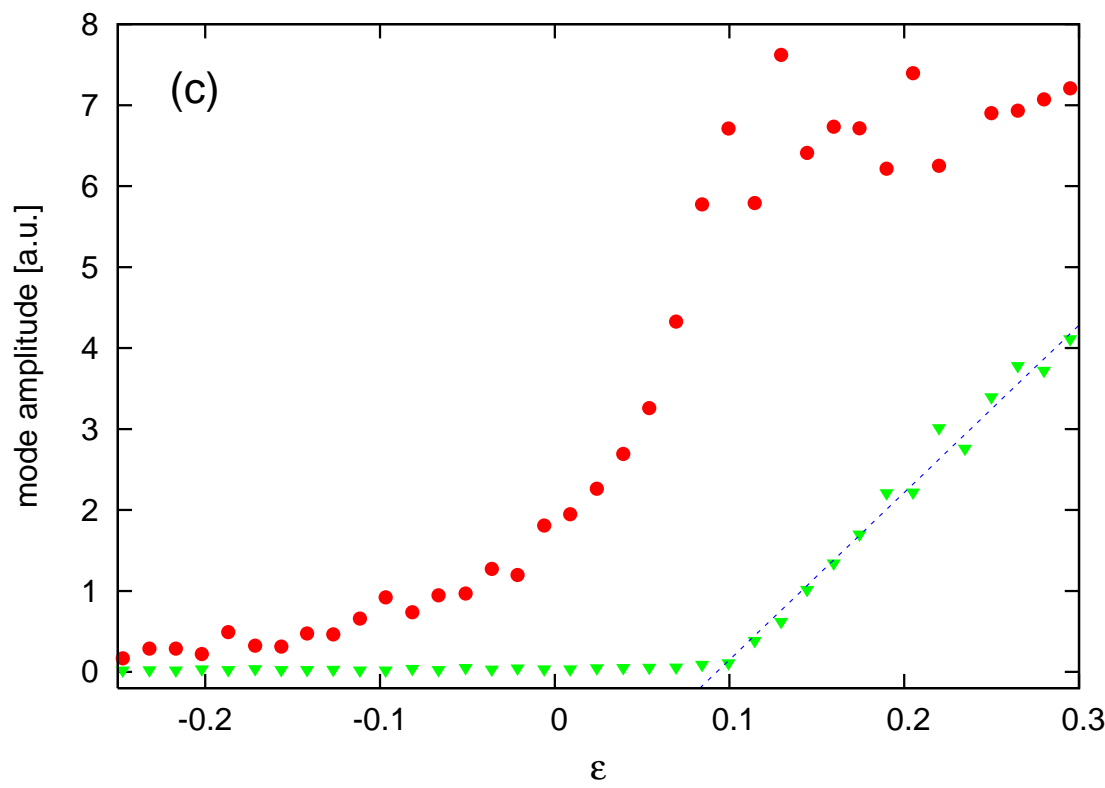

Figure 5.10: Cross roll pattern (a) and its Fourier transform (b). The image was taken at $q_{f}=1.39 q_{c}$ and $\varepsilon=0.21$. The red stripes mark the forcing structure.(c) shows the amplitudes of modes in the power spectrum $\|\left. F\left(\vec{q}_{f}\right)\right|^{2}(\bullet)$ and $\left|F\left(\vec{q}_{1}\right)\right|^{2}(\boldsymbol{\nabla})$ as a function of $\varepsilon$. A straight dashed line was fitted as a guide to the eyes.

As can be seen in Fig. 5.10c, the side mode $\vec{q}_{1}$ appears already for relative small $\varepsilon\left(\approx 0.1\right.$ at $\left.q_{f}=1.39\right)$ and its amplitude increases as a function of $\varepsilon$ similar to a pitchfork bifurcation. 
With increasing $\varepsilon$, the amplitude of $\vec{q}_{1}$ becomes larger, relative to that of $\vec{q}_{f}$. The bimodal pattern turns continuously to a roll pattern with a critical wavelength, whose intensity is modulated by the bottom plate texture. These rolls behave similar to the unforced rolls in the reference cell. They start to bend towards the side walls and reaches with increasing $\varepsilon$, a state of spatio-temporal chaos. In this more chaotic state one finds kink defect and also subharmonic resonances.

This transition from cross rolls (CR) to a spatio-temporal chaotic state is shown in detail in Fig. 5.11. Already at $\varepsilon=0.14$ the pattern turn slowly into rolls that are not perpendicular to the forcing texture but tend to bend to the side wall. At $\varepsilon=0.33$ in some areas (lower left corner) a kink structure appears while in large parts of the cell rolls various rolls exist. The kink structure becomes more pronounced at $\varepsilon=0.41$ and at $\varepsilon=0.60$ in addition areas with subharmonic resonances occur. For even higher $\varepsilon$ the pattern becomes spatio-temporal chaotic and consists of targets, short spirals and subharmonic resonances.
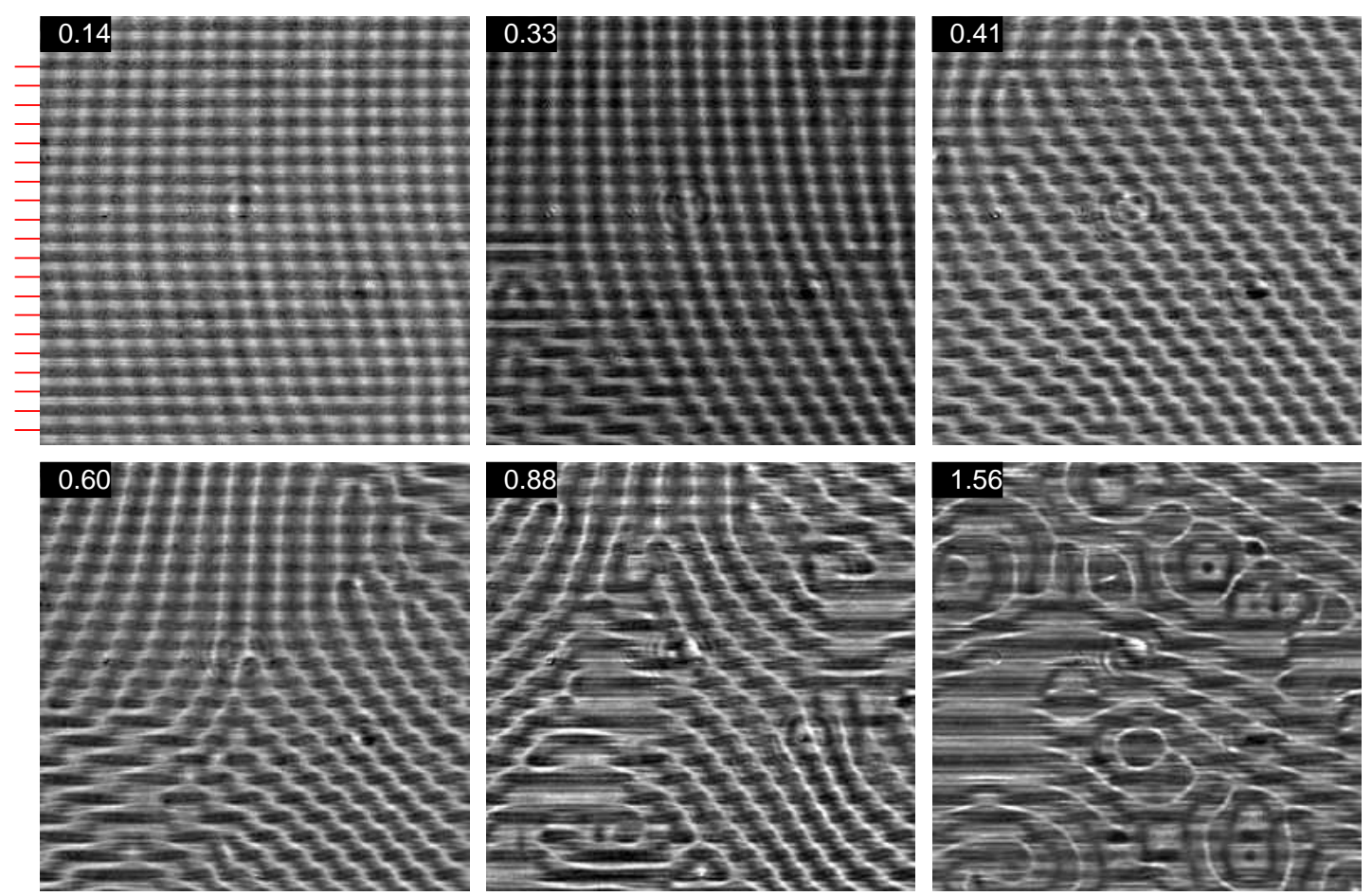

Figure 5.11: Transition from cross rolls to spatio-temporal chaos at $q_{f}=1.39 q_{c}$. The numbers on top of each image mark the values of $\varepsilon$ at which the image was taken. The red stripes mark the forcing texture at the bottom plate. Each image shows the same section of the cell with half the cell size. The section is not directly in the center of the cell but closer to the right boundary. That is why the pattern tend to bend to the right.

The forcing wave number here is so large that for the unforced system, no rolls 
with wave number $q_{f}$ are stable anymore (see Fig. 5.1). Therefore, forced rolls can only exist due to the forcing for Rayleigh numbers smaller than $R a_{c}(\varepsilon<0)$. For higher values of $\varepsilon$ a cross roll instability can be seen in the Busse balloon for the unforced RBC. A similar instability now creates perpendicular rolls for the forced case. One can argue that the cross rolls found here in the forced RBC is a frozen version of the cross roll instability in the unforced case.

\subsection{Brickwall Pattern}

As mentioned above, changing the forcing wavelength $q_{f}$ was done by changing the cell height. For resonant forcing $\left(q_{f} \approx q_{c}\right)$ and for forcing with slightly lower $q_{f}$, forced rolls are very stable and their instability line is shifted to very large $\Delta T$. Indeed, it was not possible to find the instability line of the forced rolls for the range $0.75 q_{c}<q_{f}<0.95 q_{c}$, since the maximal $\Delta T$ was larger than $15 \mathrm{~K}$ and could not be reached in this experiment.

For smaller $q_{f}$, the first instability of forced rolls is at much lower $\varepsilon$ and can therefore be reached. At $\varepsilon \approx 1$ forced rolls turn to a brick wall pattern as shown in Fig. 5.12. ${ }^{3}$
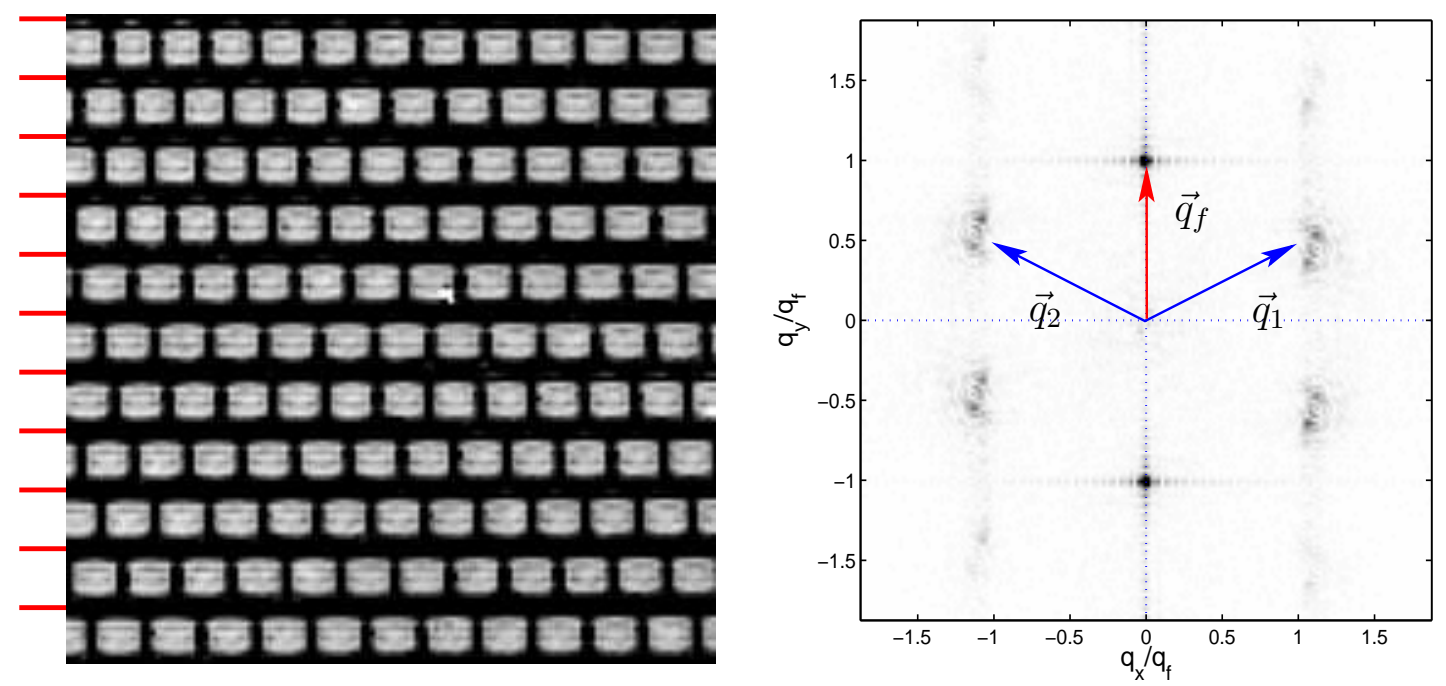

Figure 5.12: Brickwall pattern and the center part of its corresponding Fourier transform $\left(q_{f}=0.742 q_{c}, \varepsilon=0.289\right)$. The red stripes mark the forcing structure.

\footnotetext{
${ }^{3}$ Note, that all shadowgraph images shown in this section were taken with a wrong shadowgraph setting, leading to inverted images. In order not to confuse the reader the experimentally taken images were later one inverted, so that again white areas correspond to cold down-flow regions and dark areas correspond to warm up-flow regions.
} 
This pattern consists of the forced wave vector $\vec{q}_{f}=\left(0, q_{f}\right)$ and the two symmetric modes:

$$
\vec{q}_{1} \approx q_{f}\left(\begin{array}{c}
q_{x} \\
0.5
\end{array}\right) \quad \text { and } \quad \vec{q}_{2} \approx q_{f}\left(\begin{array}{c}
-q_{x} \\
0.5
\end{array}\right) .
$$

The value of $q_{x}$ decreases with $\varepsilon$ from $q_{x} \approx 1.38$ (at $\varepsilon=0.04$ ) to $q_{x} \approx 1.19$ (at $\varepsilon=0.04)$. Again, here the resonate triad condition is fulfilled:

$$
\vec{q}_{f}=\vec{q}_{1}+\vec{q}_{2}
$$

In this way the brick wall pattern turn to a more honeycomb pattern for increasing $\varepsilon$, whereas each cell is squeezed and does not have equal side length anymore. Dynamic is introduced by traveling defects (movie available on enclosed CD$\mathrm{ROM})$.

In fact one has to note here, that this pattern might be a result of non-Boussinesq effects. The cell height for these experiments were $d \approx 340-400 \mu \mathrm{m}$ and the corresponding $\Delta T_{c}$ (the temperature difference at which convection occurs in the reference cell) was $\approx 5 \mathrm{~K}$. The Busse parameter at convection onset is $Q_{\text {Busse }}=$ 1.95 (for $\mathrm{d}=340 \mu \mathrm{m})^{4}$. At convection onset in the reference cell, one could observe small areas of hexagonal patterns at boundaries of domains with different aligned rolls (shown in Fig. 5.13). One can assume that the brick wall pattern is a manifestation of the fact that here a hexagonal structure, as preferred by the unforced system due to non-Boussinesq effects, and the forcing roll pattern are both realized at the same time.

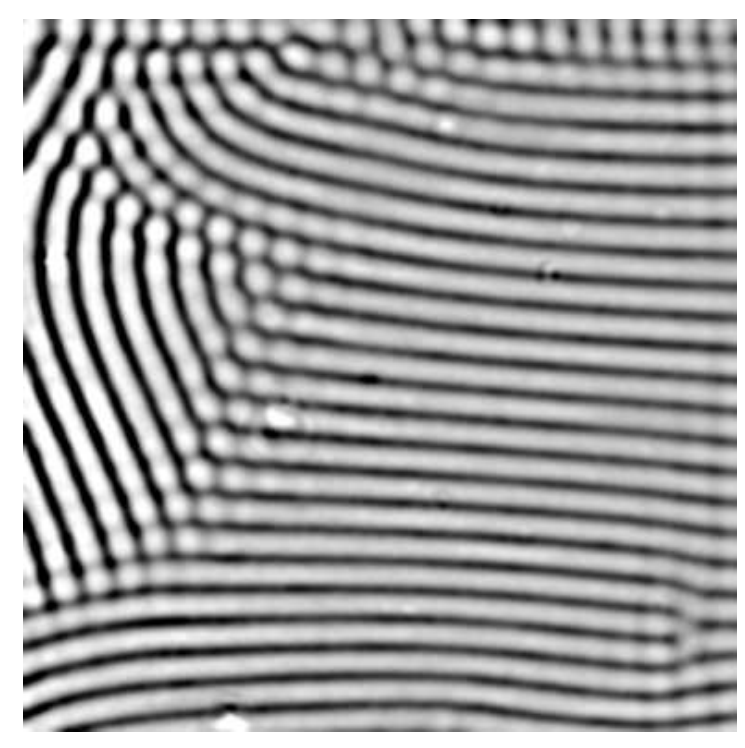

Figure 5.13: Small areas of hexagons in the unforced reference cell due to nonBoussinesq effects. The image was taken at $\varepsilon=0.05$ and with a cell height of $d=$ $370 \mu \mathrm{m}$.

${ }^{4}$ The Busse parameter is a measure for the variation of the fluid properties $(\alpha, \nu, \kappa, \varrho)$ over the cell height. It must be small to use the Boussinesq approximation.[30] 
An interesting effect could be observed for $q_{f}=0.74 q_{c}$. If one increases the control parameter, at $\varepsilon=0.054$ the forced rolls become unstable and a brick wall pattern occurs. A further increase does not lead to a chaotic state, but results in the recurrence of stable rolls with wave vector $\vec{q}_{f}$ at $\varepsilon \approx 0.45$. The evolution of brick wall pattern at $q_{f}=0.74 q_{c}$ is shown in Fig. 5.14.
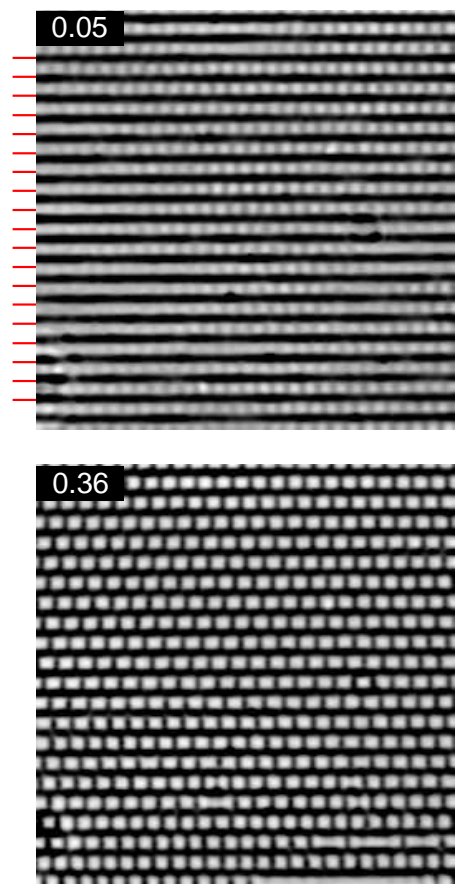
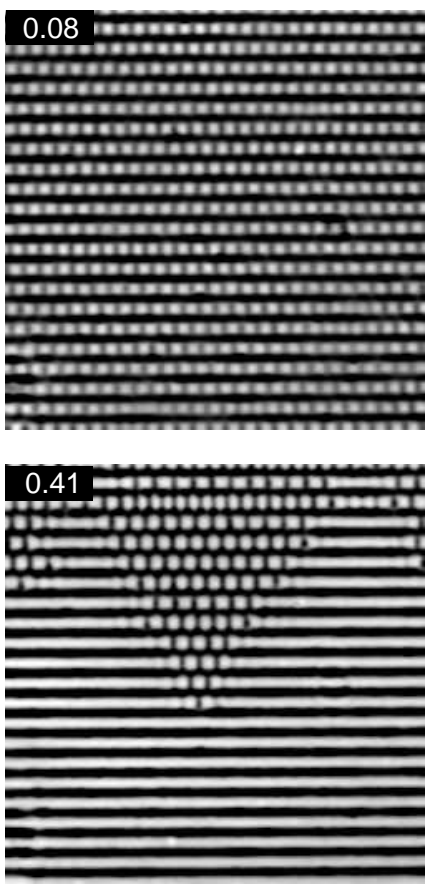

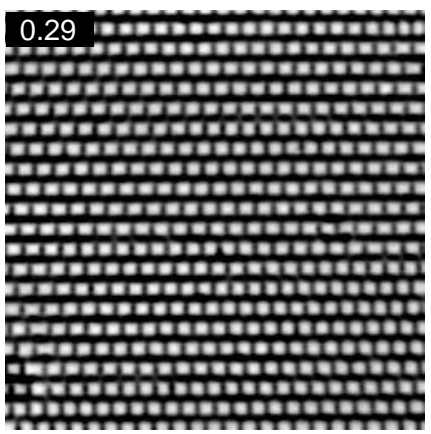

\subsection{8}

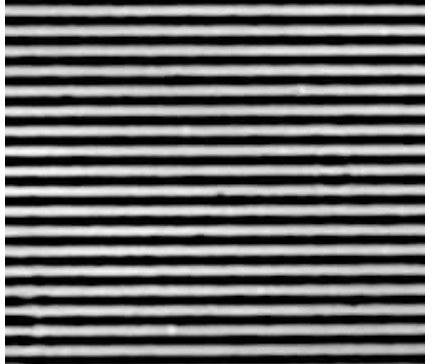

Figure 5.14: Development of brickwall pattern at $q_{f}=0.74 q_{c}$ in the range $0<\varepsilon<$ 0.48 . Numbers in the upper right corner are values of $\varepsilon$.

This recurrence of stable roll pattern is not surprising anymore if one looks at the Busse balloon in Fig. 5.1. At $\varepsilon \approx 0.4$ the region starts where rolls with wave number $q=0.74$ are stable.

\subsection{The Phase Diagram}

The different patterns as described in the previous sections are presented in the $q_{f}-\varepsilon$ phase plot in Fig. 5.15. Every symbol represents a point, where a certain pattern exist. Forced straight rolls with wave vector $\vec{q}_{f}$ are marked by $\bullet$. The instability line of straight rolls is marked by a solid black line as a guide to the eye.

Numerical analysis of forced RBC were carried out by W. Pesch and G. Freund at the University of Bayreuth [73, 98]. With their code, the instability onset of 
straight rolls was calculated. The change in the forcing parameter $\delta$ due to a change in the cell height was taken into consideration. The predicted onset is plotted as a brown curve in Fig. 5.15.

As can be seen, the theoretical prediction agrees qualitatively with the experimental measurements. For example, the recurrence of straight rolls at $q_{f}=0.742 q_{c}$ does also occur in the numerical calculations even though for lower forcing wave numbers $0.45 \lesssim q_{f} \lesssim 0.70$. Also the strong increase of the stability line for $q_{f} \leq q_{c}$ is observed in the simulation as well as the decrease to $\varepsilon=0$ for $q_{f}>q_{c}$.

Five different instabilities of the straight rolls could be observed in the simulations. These instabilities are marked with capital letters in the inlet in Fig. 5.15. For $q_{f}<3 / 4 q_{f}$ the simulation predicts an instability at $\varepsilon \approx 0(\mathrm{~A})$. The destabilizing modes are characterized by $\left|\vec{q}^{\ddagger}\right|=q_{c}$ and $q_{y}^{s}=q_{f} / 2$. The symmetric modes of the brick wall pattern fulfill these conditions. In the vicinity of $q_{f} \approx 3 / 4 q_{c}$ the simulation shows that the forced rolls are very stable and are confined by the instability marked with (B) in Fig. 5.15. These instabilities are predicted to have wave vectors with $\left|\vec{q}^{s}\right| \approx 3 / 4 q_{c}$ and $q_{y}^{s}=0$. This boundary could not be explored experimentally due to limitation of the maximal reachable temperature difference of $\Delta T_{\max } \approx 15 \mathrm{~K}$. In the range of $q_{f} \approx q_{c}$ the upper instability (marked with $\mathrm{C}$ ) is predicted to have instability vectors $\vec{q}^{s}$ with $q_{c} / 2<\left|\vec{q}^{s}\right|<q_{c}$ and with an oblique angle so that the y-component is $q_{f} / 2<q_{y}^{s}<q_{c}$. This was also seen in the experiment by the varicose pattern. Unfortunately, the kink lines cannot be seen by these instability modes. Single kinks can only be explained by very short $\vec{q}^{s}$. For $q_{f}>1.3 q_{c}$ the simulation predicts an instability onset again close to $\varepsilon \approx 0$ with $\left|\vec{q}^{s}\right|=q_{c}(\mathrm{E})$, which is also given by the cross rolls shown above. In a very small region close to $q_{f} \approx 1.2 q_{c}$ (D) the instability sets in for $\varepsilon>0$ and has instability modes of $\left|\vec{q}^{s}\right|=q_{c}$.

As promising as the qualitative agreements are, there is no quantitative agreement between experiment and simulation. In general, the theoretical curve is shifted to smaller $q_{f}$ by approximately $s_{q_{f}} \approx 0.15 q_{c}$. But also a simple shift cannot explain the difference between theory and experiment. Especially for $q_{f} \approx q_{c}$, the experimentally measured instability line is much higher (larger $\varepsilon$ ) than the theoretical prediction.

One could argue, that the forcing parameter $\delta$ is underestimated in the theory, since theory maps the boundary modulation to a modulation of the temperature at the bottom plate, and thus only one forcing mechanism is taken into consideration instead of two (boundary forcing of the velocity and the temperature field). However, this is not the case. An increase in $\delta$ would blow up the whole area where rolls exist in all directions. As an example, for $q_{f}=1.01 q_{c}$ the instability occurs in the experiment at $\varepsilon_{e}=3.42$ whereas theory predicts $\varepsilon_{t}=1.292$. In order to reach the experimental values in the simulation, $\delta$ has to be increased by a factor of 10 (from $\delta=0.02548$ to $\delta=0.275$ ). On the other hand, if one assumes 


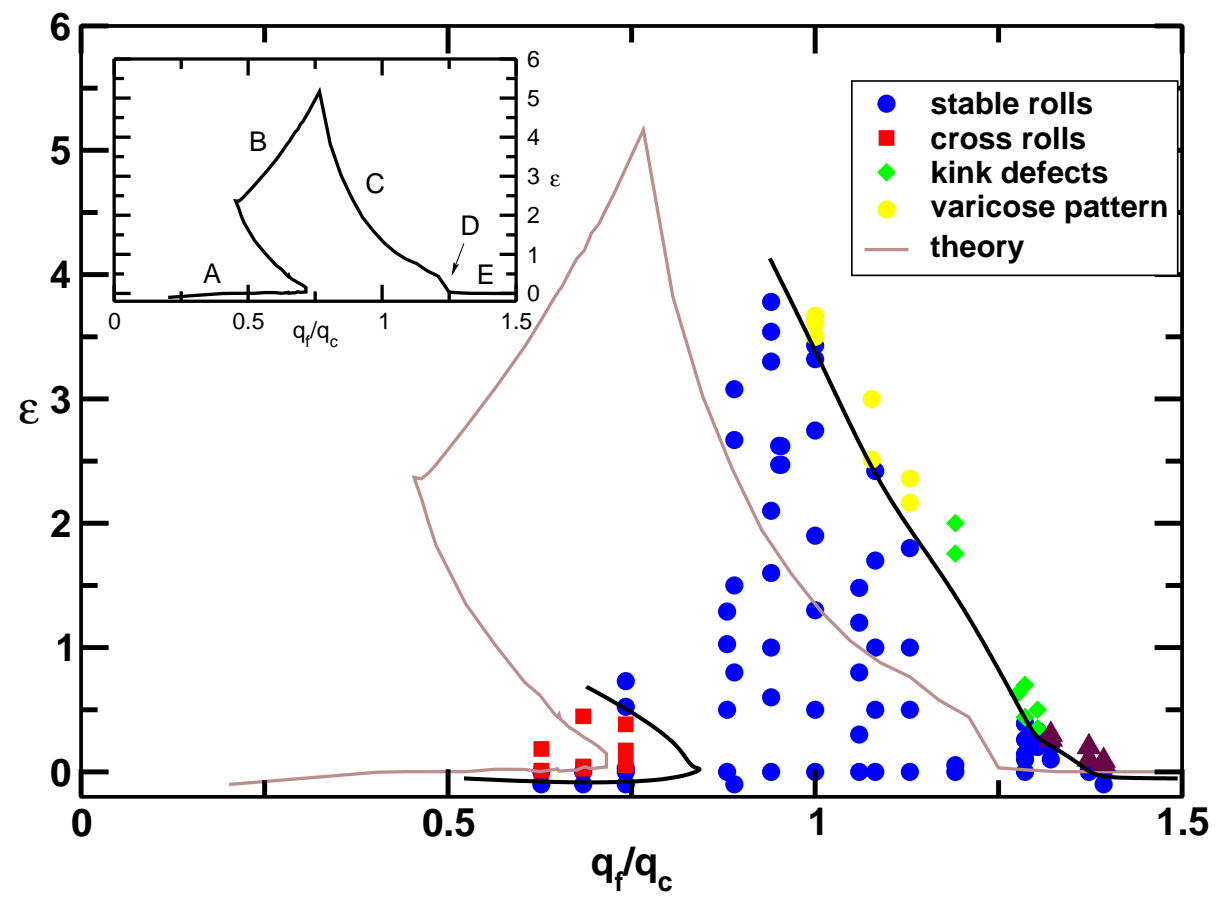

Figure 5.15: Instability of forced rolls with various wave numbers. The points mark certain pattern found in experiments. The black solid line is a hand drawn guide to the eye and marks the first instability of forced rolls. The brown line shows the numerical calculated instability line. The inlet shows the same theoretically predicted curve and marks several instabilities with letters $\mathrm{A}$ to $\mathrm{E}$ as explained in the text. 
$\delta=0.275$ and calculates the instability line for $q_{f}=0.685$, one would get a huge value of $\varepsilon_{t}>10000$. Therefore an over- or underestimation of $\delta$ cannot be the reason for the discrepancy between theory and experiment.

An important point to note is the shift of $s_{q_{f}} \approx 0.15 q_{c}$ between theory and experiment, that corresponds to a shift in the cell height of $s_{d}=75 \mu \mathrm{m}$, which is approximately the height of the SU-8 ridges. It might be, that the cell height in the theory is for some reason underestimated by approximately the height of the SU-8 texture. 


\section{Chapter 6}

\section{Optical Forcing - An Outlook}

Forcing by surface corrugation as presented in this thesis was a convenient and precise approach to investigate the effect of spatial forcing on a thermal convection system. The height and width of the corrugation could be measured very precisely and since SU-8 is a often used photoresist, values for its heat conductivity can be found in literature. However, this approach has several disadvantages. In example, the corrugation of the bottom plate influences not only the temperature distribution at the bottom plate surface, but also the boundaries of the flow. From the pure experimental point of view this is not a problem. But in order to make theoretical predictions which are comparable to experimental results, one is usually interested in "easy-to-describe" boundaries. In order to see the influence of specific parameter to the system, it is necessary to modify as few parameter as possible. From the experimental point of view the biggest disadvantage of a modulated bottom plate is the influence to the shadowgraph signal. It is not known, how to remove this effect by image processing in order to get only the shadowgraph signal of the convective flow.

Therefore, an improvement would be optical forcing. This for example, can be done by light, which is absorbed by the working fluid. In this way energy can be deposited in the fluid in a well controlled manner and also in an almost arbitrary spatio-temporal distribution. With this approach one doesn't only get rid of an influence in the shadowgraph, but one can also easily vary the spatial intensity of energy deposition. Producing a convenient mask is orders of magnitude cheaper and less time consuming. By moving the mask one can even easily force traveling waves and other time dependent structures. Beside for research of pattern formation, experiments on internal heating are also interesting on their own, since several convection processes in nature are driven by an internal heating of the convective fluid. Examples include heat production by radioactive decay inside the earth mantle which forces mantle convection or the release of latent energy due to cloud condensation inside the atmosphere (see e.g. [99]). 
While the effect of internal heating on thermal convection was studied extensively in theory and simulations (see e.g. $[100,101])$, experiments are very rare. Several of these experiments were done by Busse and Whitehead and Chen [36, 35, 38] in order to test the stability of longitudinal rolls in Rayleigh-Bénard convection and thus determine the stability boundaries of the Busse-balloon. They used water as working fluid and forced rolls via a metal template and a 500 Watt light bulb. The light shone onto the mask which produced a one-dimensional stripe pattern onto the water. Due to absorption of the infrared part of the light by the water, the water was locally heated. This perturbation was sufficient to force rolls with a wavelength given by the metal template.

Our goal was to use the same technique in order to force rolls, hexagons and other symmetric structures with a specific wave number. The experimental setup and preliminary results are presented in the following sections.

\subsection{Theory}

Theoretical considerations on internal heating can be found in [102] and [100]. In comparison to the forcing with surface corrugation via the bottom plate boundary, the forcing here enters in the heat equation (Eq. 2.3). An energy source term has to be added which include the energy deposition rate $Q(x, y, z)$ :

$$
\frac{\partial T}{\partial t}+(\vec{v} \nabla T)-\kappa \Delta T=\frac{Q}{\varrho_{0} c_{p}} .
$$

Here, $\varrho_{0}$ and $c_{p}$ denote the averaged density of the fluid and its heat capacity. Let us assume forcing of a stripe pattern. The energy deposition due to light absorption decreases exponentially in $z$ with absorption coefficient $\mu$ and is periodically modulated in $x$-direction :

$$
Q(x, y, z)=\mu I_{0} \exp (\mu(z-d / 2)) \cdot\left(1+a \cos \left(q_{f} x\right)\right) .
$$

The initial light intensity is given by $I_{0}$, the forcing wavelength is $q_{f}$ and $a$ is the modulation amplitude. The boundaries are both held at constant temperature:

$$
T(z=d / 2)=T_{t} \quad \text { and } \quad T(z=-d / 2)=T_{b} .
$$

Together with this boundary condition the solution of the inhomogeneous heat equation (Eq. 6.1) for the pure steady conduction case $\left(\partial_{t} T=0\right.$ and $\left.\vec{v}=0\right)$ is the sum of the general homogeneous solution and a particular solution of the inhomogeneous equation:

$$
T_{\text {cond }}(x, y, z)=T_{\text {hom }}(z)+T_{\text {in }}(x, y, z) .
$$


Here, $T_{h o m}$ is already known from Chapter 2 to be:

$$
T_{\text {hom }}=T_{b}+\beta(z+d / 2) ; \quad \beta=\frac{T_{b}-T_{t}}{d} .
$$

Since the homogeneous solution already fulfills the boundary condition at the top and the bottom plate, $T_{i n}$ has to be 0 at $z= \pm d / 2$. An inhomogeneous solution is for example:

$$
T_{i n}(x, z)=A_{0} \cdot\left(T_{m}^{0}(z)+a T_{m}^{1}(z) \cos \left(q_{f} x\right)\right) \quad \text { with } \quad A_{0}=\frac{\mu I_{0}}{\varrho_{0} c_{p}} .
$$

For lateral homogeneous heating $(a=0), T_{i n}$ can easily be found just by integrating Eq. 6.1:

$$
T_{\text {in }}(z)=-\frac{I_{0}}{\varrho_{0} c_{p} \mu}\left[e^{\mu(z-d / 2)}+e^{-\mu(z+3 d / 2)}-2 e^{-\mu d}-(z / d+1 / 2) \cdot\left(1-e^{-\mu d}\right)^{2}\right] .
$$

(a)

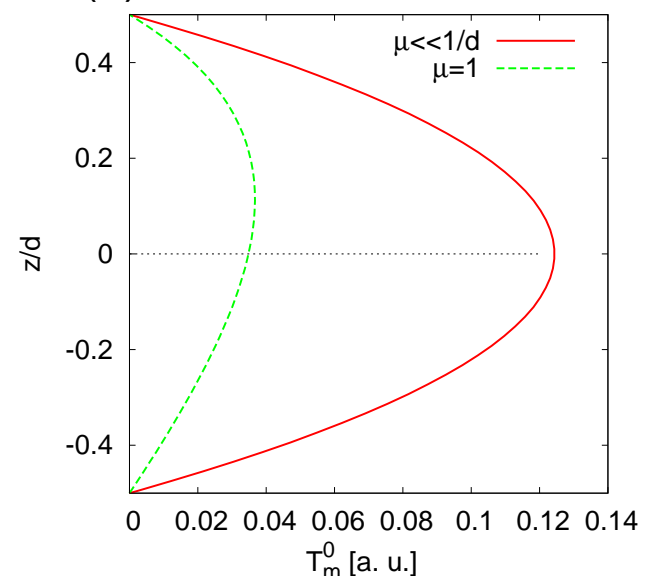

(b)

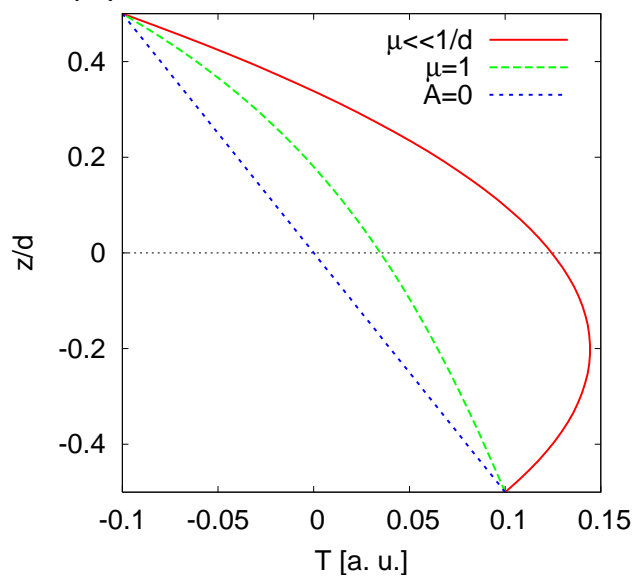

Figure 6.1: Temperature profiles along the cell due to internal heating. Left plot (a) shows the inhomogeneous solution $T_{i n}$, while (b) shows the full temperature profile in the conduction state for various absorption coefficients $\mu$.

The resulting temperature profiles for the conduction state are plotted in Fig. 6.1. If the absorption coefficient is sufficiently small in comparison with the inverse of the cell height ( $\mu \ll 1 / d$, homogeneous heating), the amount of energy deposit in the fluid is independent of the z-coordinate. The red line in Fig. 6.1 marks this 
case. A maximum of the temperature occurs in this case at around $z=-0.2$. Hence, close to the bottom plate a stable layer occurs, where warmer fluid is on top of colder fluid. On the other hand the gradient of the unstable layer $(-0.2<z<0.5)$ is increased which destabilizes the fluid. This effect reduces the critical temperature difference at which convection sets in. In the other case, when $\mu$ is sufficiently large, heating takes place in the upper part of the cell the maximum of $T_{i n}$ is at positive $z$ (green dashed line in Fig. 6.1a). In this case areas closer to the cold top plate are heated and the critical temperature difference at which convection sets in is increased. For homogeneous internal heating $(\mu \ll 1 / d)$ the critical Rayleigh number is reduced, while for larger absorption coefficient $R a_{c}$ is increased (see [100]).

\subsection{The Experiment}

The setup for this experiment was basically the same as for forcing via a textured bottom plate. It is also worth noting here the work of Gabriel Shaughnessy,[103], who designed an analog optical forcing experiment. As shown in Fig. 6.2 between the shadowgraph tube and the main pressure vessel, a wavelength sensitive mirror was installed, which was transparent for the visible light and reflective in the IR-range. A $600 \mathrm{~W}$ light bulb was used as an IR source. The light was filtered with a thin silicon wafer of $0.5 \mathrm{~mm}$ thickness in order to remove the visible part of the spectrum. The working gas was $\mathrm{CO}_{2}$, which absorbs electromagnetic radiation with a wavelength of $\lambda \approx 4.8 \mu \mathrm{m}$ and $\lambda \approx 2.7 \mu \mathrm{m}$ [104]. Since water strongly absorbs IR radiation, it could not be used as a cooling liquid. Sulfur hexafluoride $\left(\mathrm{SF}_{6}\right)$, which is practically transparent at the above lines, was used instead. This inert gas, usually used in electrical applications, becomes liquid at room temperatures at around 20 bar.

The IR radiation and thus the spatial distribution of heat deposition inside the fluid was modulated by using a mask (see Fig. 6.2). A CaF-lens (glass is not transparent for light with $2.5 \mu \mathrm{m}$ ), images the mask on the fluid and produces in this way a spatial periodic distribution of light intensity on the fluid. Unfortunately the temperature dependency of the refractive index of liquid $\mathrm{SF}_{6}$ is much stronger than for water and thus the cooling flow gives and additional random shadowgraph signal. By averaging over 10 images for each measurement this effect was reduced. This procedure worked well but nevertheless the noise level (standard deviation of the gray value for the pure conducting state) was significantly higher than for the case where water was used as a cooling liquid.

Results presented in this chapter are due to experiments at room temperature $\left(T_{a v}=25^{\circ} \mathrm{C}\right)$ and at a pressure of $41.4 \mathrm{bar}(600 \mathrm{psi})$ resulting in a Prandtl number of $\operatorname{Pr}=1.1$. The cell height was $d=945 \mu \mathrm{m}$.

A power meter was used to measure the energy input in the cell. In fact, the 


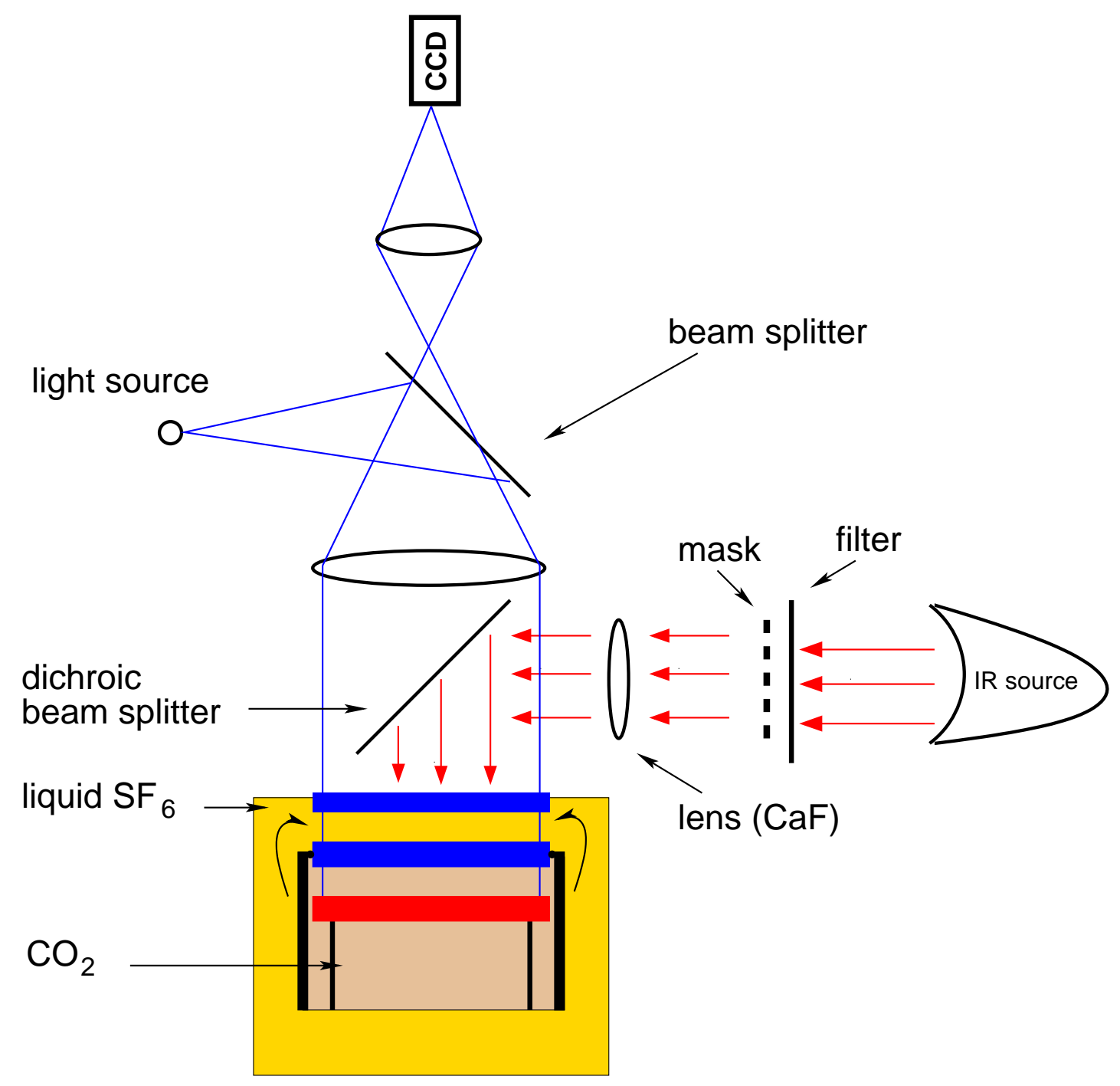

Figure 6.2: Experimental setup of an optical forcing experiment. 
values were measured outside the cell and the energy input in the cell was later on calculated by assuming reflection and absorption at the beam splitter and both sapphire plates. The value for the intensity modulation as defined in Eq. 6.2 was measured to be $a=0.2$. The input energy flux was $I_{0} \approx 5 \mathrm{~mW} / \mathrm{cm}^{2}$. This is only a very rough approximation and was calculated by the power meter measurements and the assumption of $90 \%$ loss of energy due to reflection at optical interfaces and absorption in the sapphires (see [103] for details). As will be shown in the next chapter, this energy is probably overestimated, since the response in the actual experiments was very small. The value for the absorption coefficient of $\mathrm{CO}_{2}$ for the bigger $2.7 \mu \mathrm{m}$ band is $\mu=6.84 \mathrm{~cm}^{-1}$ ([104] and [105]).

\subsection{Results and Discussion}

\subsubsection{Spatial Periodic Forcing and the Amplitude Equa- tion}

During this experiment the mask was imaged via CaF lenses onto the cell. It was difficult to determine the exact image distance and therefore one does not know the exact forcing wave length. But by trial and error the image distances were chosen so that the responds of the system were maximal. Thus, one assumes the forcing wave length to be the critical wave length $\left(q_{f}=q_{c}\right)$.

The development of the amplitude for the forced and the unforced cases are shown in Fig. 6.3. Note that the basic noise level is very high due to the strong temperature dependency of the refractive index of the cooling liquid.

The fit in Fig. 6.3 is again a cubic amplitude function similar to Eq. 2.25:

$$
\varepsilon A-g_{0} A^{3}+c=0 .
$$

The value of $g_{0}$ is the same as for boundary forcing and it was assumed that the amplitude of the shadowgraph signal $A_{s}$ is proportional to the convection amplitude. This assumption is valid if the forcing strength is very small as shown in Eq. 4.3. And indeed, the fitted value of $c$ is:

$$
c=1.04 \text {. }
$$

This value is almost an order of magnitude lower than the constant term in the boundary forcing case, where $c=g_{2} \cdot \delta_{f i t}=240 \cdot 0.032=7.68$. Considering forcing power and modulation amplitude as stated in the previous paragraph, one would have in the cell alternating stripes with $Q=0.2 .5 \mathrm{~mW} / \mathrm{cm}^{2}=1 \mathrm{~mW} / \mathrm{cm}^{2}$. This is 


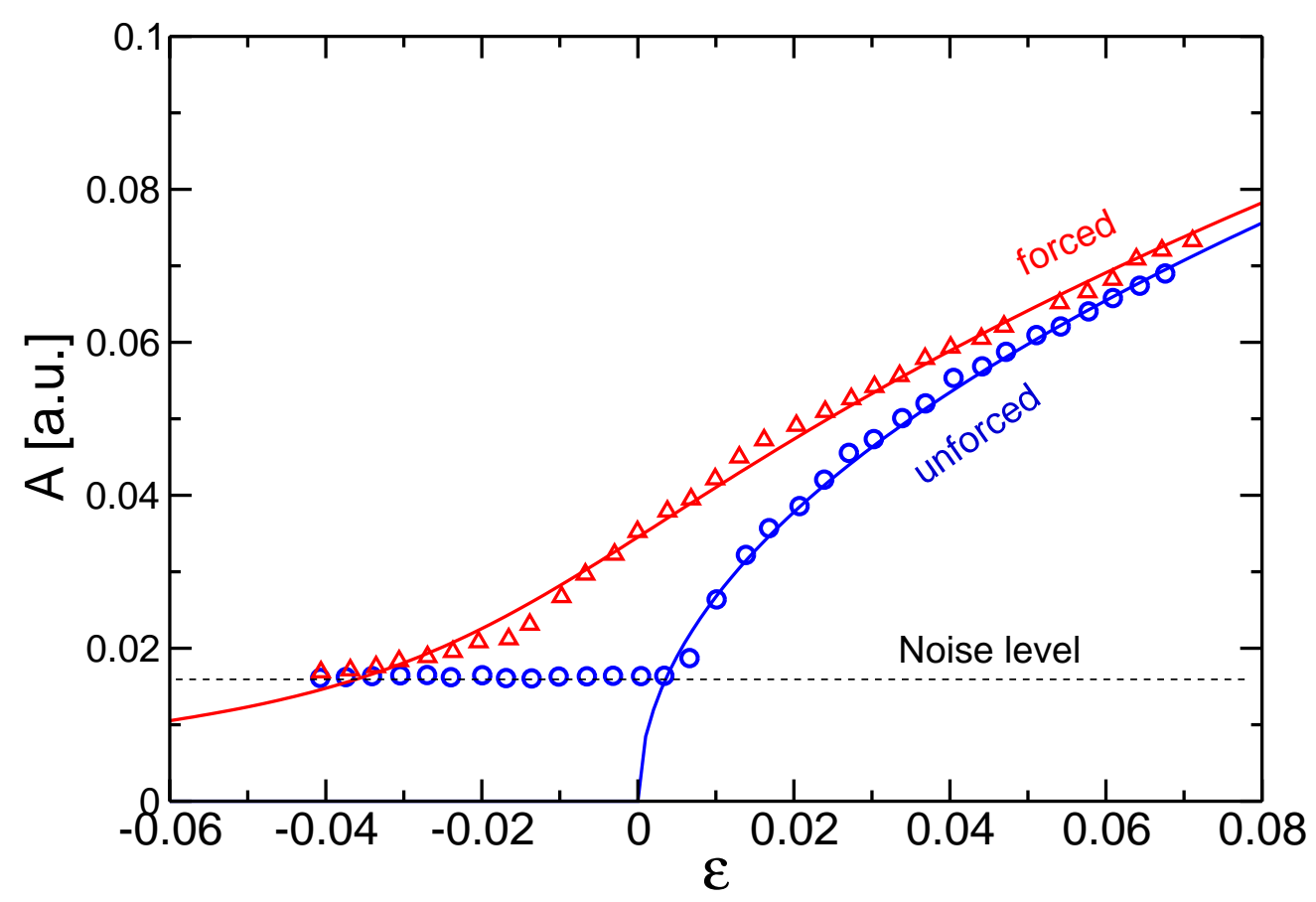

Figure 6.3: Imperfect bifurcation due to spatial periodic internal heating. Red triangles mark amplitude development in the presence of periodic forcing. Blue circles are the amplitude without forcing. The solid red and blue lines represent the corresponding fitted curves. The green line marks the theoretically predicted curve. The dashed black line shows the level of noise due to flow of the cooling liquid. 
the same order of magnitude as the energy flux through the cell due to conduction at convection onset (values from [82]):

$$
j=\kappa \cdot \frac{\Delta T}{d}=2.130 \cdot 10^{-1} \mathrm{~mW} / \mathrm{s} \mathrm{cm} \mathrm{K} \cdot \frac{0.595 K}{0.0945 \mathrm{~cm}}=1.34 \mathrm{~mW} / \mathrm{cm}^{2} .
$$

Thus, it is very surprising, that the constant term in Eq. 6.3 is so low. It is highly probable, that the actual energy input is much lower than calculated.

Even though, a quantitative agreement between theory [102] and experiment could not be achieved, once again the occurrence of an imperfect bifurcation due to periodic forcing was found.

\subsubsection{Forcing Close to $R a_{c}$}

It turned out that by using a $600 \mathrm{~W}$ black body radiator the energy input was to weak. One only could force patterns with the critical wavelength and very close to the convection onset. The system did not respond to forcing with other wavelengths or at higher $\varepsilon$. If one tried to imprint a two-dimensional pattern into the system, the pattern turned immediately to straight rolls if the control parameter was larger as $\varepsilon=0.015$. In general the imprinted pattern with wavelength $\lambda_{c}$ could be observed in the range of $-0.05<\varepsilon<0.015$.

A way to imprint a convective pattern with high convection amplitude is to ramp up the temperature difference very fast. In this way convection starts in the sub-critical region and is driven by a very localized temperature gradient. The information about a state at point $A$ needs a certain time to diffuse in lateral direction to point $B$. A typical time scale can be given by $L^{2} / \kappa$ with $L$ being the spatial distance between $A$ and $B$ and $t_{\kappa}$ being the thermal diffusion time. The effect of such a ramp is shown in Fig. 6.4.

The response of the convection flow to the temperature ramp is shown in Fig. 6.4. Ramping in this experiment was done by setting a constant voltage (constant heating power) for approximately $7 \mathrm{sec}$ to the bottom plate heater, while the top plate temperature was held constant. The development of the bottom plate temperature, measured at its bottom side is plotted versus time in Fig. 6.4b. Note that this procedure was not started at $\Delta T=0 K(\varepsilon=-1)$. The response of the fluid is delayed due to the finite heat conductivity of the silicon plate and the working fluid. Assuming a thermal diffusion coefficient for silicon of $\lambda_{S i}=8.8 \cdot 10^{-5} \mathrm{~m}^{2} / \mathrm{s}$ and a silicon plate of thickness $d=9.5 \cdot 10^{-3} \mathrm{~m}$, the characteristic diffusion time for the heat to travel through the bottom plate becomes $t_{h}=1 \mathrm{~s}^{1}$. Therefore, the delay between measured temperature difference and applied temperature difference is 1 sec. If one measures a specific temperature

\footnotetext{
${ }^{1}$ Assuming $\lambda=\frac{\kappa}{\varrho c_{p}}$ with $\kappa=145.7 \mathrm{~W} / \mathrm{m} \cdot K$ [76], $\varrho=2.33 \mathrm{~g} / \mathrm{m}^{3}$ [106] and $c_{p}=712 \mathrm{~J} / \mathrm{kg} \cdot \mathrm{K}[106]$.
} 
(a)
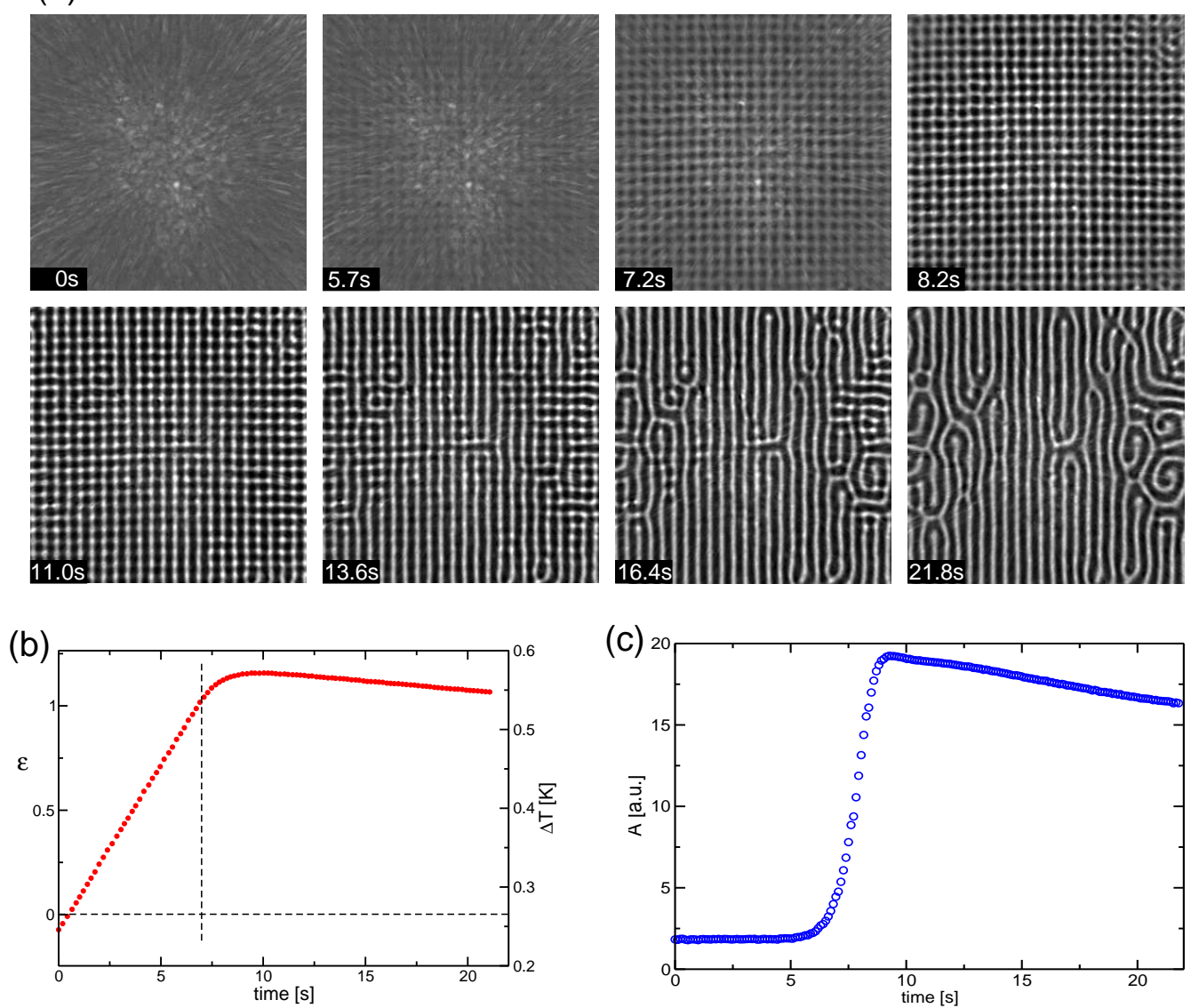

Figure 6.4: Sequence of shadowgraph images taken during a fast increase of the temperature difference (a). The plots show the measured temperature difference and calculated control parameter as a function of time (a) and the development of the convection amplitude as a function of time (c). The horizontal dashed line in (b) marks the onset of convection in an unforced experiment. The vertical dashed line marks the time at which the bottom plate heater was switched off. 
difference, the fluid will "feel" the same difference approximately $1 \mathrm{~s}$ later. The characteristic heat diffusion time through the $\mathrm{CO}_{2}$ is around $t_{\kappa}=10.7 \mathrm{~s}$ ([82]). That means, after changing the temperature difference in the purely conducting state it takes $10.7 \mathrm{~s}$ to build a new profile.

Figure 6.4a shows shadowgraph snapshots of the convective flow. At $t=0 \mathrm{~s}$ one sees the turbulent motion of the cooling fluid $\left(\mathrm{SF}_{6}\right)$ but no convection pattern. At $t=5.7 \mathrm{~s}$ the imprinted square pattern becomes visible. From experiments with an adiabatic (slow) increase in temperature difference, one knows that forced rolls occur at around $\varepsilon=-0.05$, meaning their amplitude is equal to the noise amplitude of the cooling liquid ${ }^{2}$. This value is measured after $0.5 \mathrm{~s}$, so the time delay to the onset is about $5.2 \mathrm{~s}$. Thus, the instability to convection does occur even before the new temperature profile is fully developed. The mask we used for this experiment consists of little holes, sitting on a squared lattice. Therefore heating occurs point wise resulting in a convection pattern whereas dark points (hot rising fluid) is surrounded by white borders (cold sinking fluid).

Note that the maximum of the $\varepsilon$-curve (Fig. $6.4 \mathrm{~b}$ ) occurs $1 \mathrm{~s}$ earlier than the maximum of the amplitude curve (Fig. 6.4c) corresponding to the heat diffusion time through the silicon bottom plate. Here the heat diffusion time through the fluid layer doesn't play a roll since the fluid is already in motion and information there travels much faster. If one lowers the surface temperature of the bottom plate the velocity of the fluid will slow down immediately.

Convection takes place with increasing amplitude, following the imprinted square structure with critical wavelength $\lambda_{c}$. After around $11 \mathrm{~s}$, the bottom plate temperature decreases and the imprinted squares turn slowly to rolls. Squared solutions are unstable and can only exist in the vicinity of $\varepsilon=0$. Rolls do occur if interaction takes place between neighboring squared convection cells. This is usually the case after a characteristic time scale on which momentum diffuses - the viscous diffusion time. In our case this time is $t_{\nu}=9.7 \mathrm{~s}$ which roughly corresponds also with the observation, that after $14 \mathrm{~s}(8 \mathrm{~s}$ after the first convection were observed) most of the area is covered with convection rolls instead of squared pattern. Finally - $22 \mathrm{~s}$ after starting the ramp - the system reaches a state of straight rolls with several defects. In this state, the forcing does not seem to have any influence anymore on the roll pattern. Even if one lowers $\varepsilon$ significantly, the forcing influence seems not to be significant to the pattern shape. Only if one reaches $\varepsilon=0$ squares occur again.

Beside squares also other pattern were imprinted as shown in Fig. 6.5. Shown here are images of the convective flow (top row), the center area of the Fourier transform (middle row), and a sketch of the grid that was used for forcing (bottom

\footnotetext{
${ }^{2}$ Onset in the sense that the shadowgraph signal due to convection is visible in the Fourier spectra and therefore of equal strength as the noise due to the turbulent cooling flow. As mentioned earlier there is no onset for forced convection anymore.
} 
row). White areas in the grid mark transparent areas (holes) that correspond with local heating of the fluid, leading to rising flow at that points. This produces dark areas in the shadowgraph image.

Column (a) shows the same square forcing experiment as described above. The flow pattern is constructed by two vertical modes, both having critical wavelength in complete correspondence with the forcing grid. In column (b), a rhombic pattern was forced. It is interesting that while in the vertical forcing case one really sees a superposition of rolls, here instead one has small jumps in the phase of the pattern. In column (c) a hexagonal pattern was forced and appears also as convection pattern. Only the quality of the image is reduced by the strong cooling flow of $\mathrm{SF}_{6}$. An interesting phenomenon occurs in column (d). Here again a bimodal pattern was forced in the same way as in (a), but now with a forcing wavelength of $\lambda_{f} \approx 1.33 \lambda_{c}$. Here, the response of the system is a bimodal patter, but at $45^{\circ}$ to the forcing pattern. In this way the response follows again the grid, but also has a wavelength close to the critical wavelength of the system. The actual wavelength of the pattern is therefore:

$$
\lambda=\frac{1.33 \lambda_{c}}{\sqrt{2}}=0.94 \lambda_{c}
$$

This is also shown in the Fourier transform in column (d). The red arrows mark the forcing wave vectors, while the blue arrows mark the actual response of the system. Again, similar to various pattern presented in this thesis, the system tries to build up a convection pattern containing the critical wave number as an intrinsic constituent of the system

\subsection{More Power - The Use of an IR Laser.}

Using the IR part of a black body radiator turned out not to give sufficient power in order to conduct forcing outside the Busse-balloon or further away from the critical Rayleigh number $\left(R a_{c}=1708\right)$. Even at a radiation power of $600 \mathrm{~W}$ the effective energy in the $\mathrm{CO}_{2}$ band was to small. On the other hand, a huge amount of energy was pumped into the system, absorbed by all kinds of components but the $\mathrm{CO}_{2}$. This leads to a strong heating of the bottom plate and thus the temperature of the bottom plate could not be regulated anymore since the heating by absorption was stronger than the heating by the bottom plate heater.

An alternative to a black body radiator is an IR-laser. A corresponding experiment is being designed and build and will be explained in this section. A continuous wave $\mathrm{CO}_{2}$ laser (Synrad Technology, 48-5SW) is used with an output 

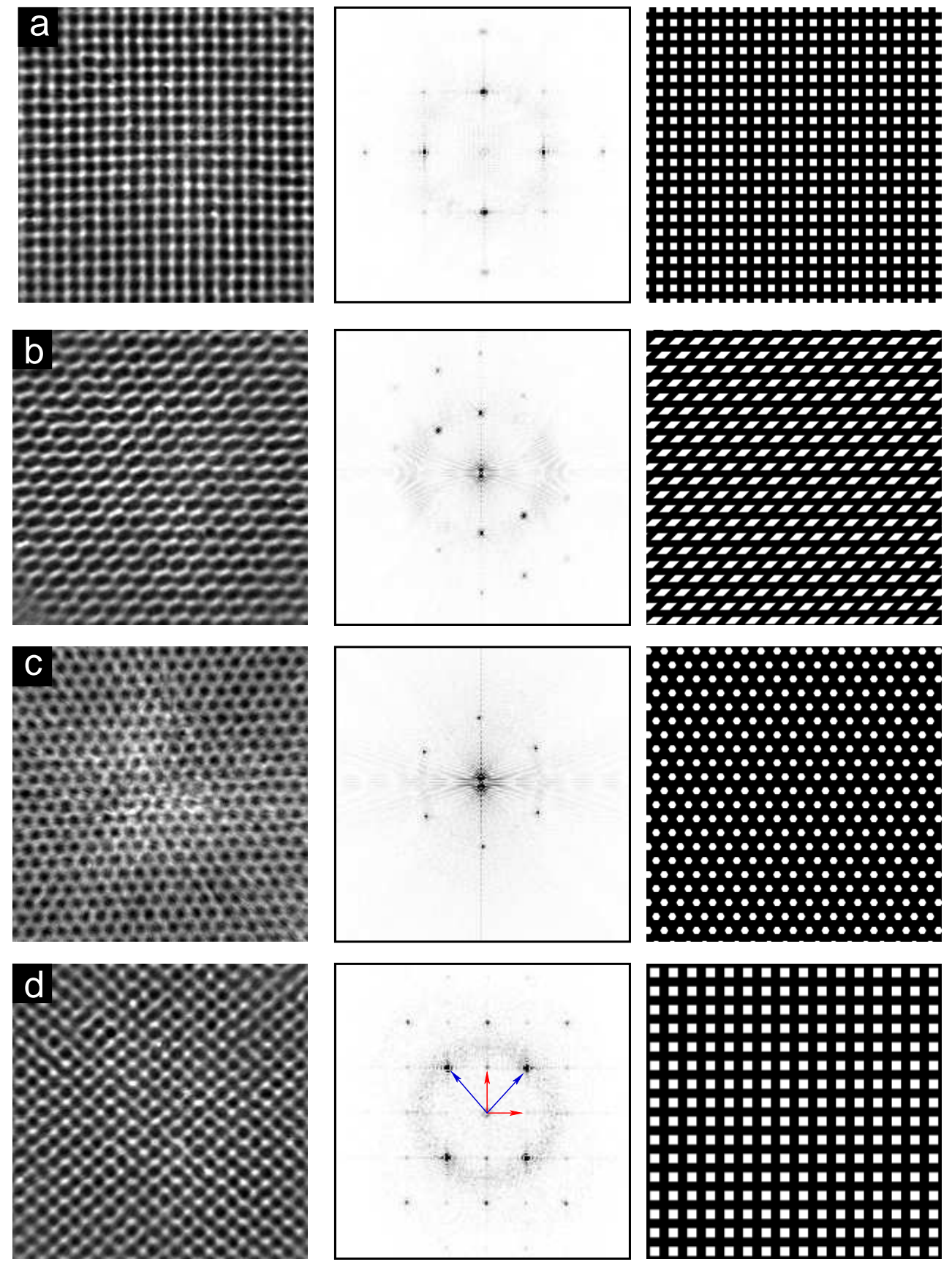

Figure 6.5: Imprinted convection pattern due to optical forcing (upper row), their corresponding Fourier transform (middle row) and the mask which was used for forcing (lower row). First column (a) shows the bimodal pattern mentioned above. (b) shows a rhombic pattern with two modes, (c) shows a hexagonal pattern and (d) shows a oblique squared pattern due to square forcing with a wavelength of $\lambda_{f} \approx 1.33 \lambda_{c}$. 
of $60 \mathrm{~W}$ at a wavelength of $\lambda=10.6 \mu \mathrm{m}$. Fortunately this wavelength corresponds exactly with the absorption wavelength of $\mathrm{SF}_{6}$, a gas which is already used in various RBC experiments (e.g. [107, 108, 68]) and whose properties are well known. It was harder to find an appropriate cooling liquid for our apparatus. Water cannot be used anymore since it absorbs strongly at a wavelength of $10.6 \mu \mathrm{m}$. Prof. Michael Schatz (Georgia Tech) suggested the use of carbon disulfide $\left(\mathrm{CS}_{2}\right)$, a poisonous liquid under standard conditions which is transparent to electromagnetic radiation of about $10 \mu \mathrm{m}$. $\mathrm{CS}_{2}$ is not only a poison but also highly flammable and that is why significant care has to be taken while dealing with it.

In addition to the working fluid and cooling liquid the top pressure window and the top plate have to be changed from sapphire, which is not transparent for this wavelength, to zinc selenide (ZnSe). The ZnSe windows (purchased by ISP optics) were covered with an additional anti-reflection coating in order to increase their transparency for the IR-radiation from $65 \%$ (without coating) to $97 \%$ (with coating). The usually transparent silicon bottom was coated with a thin gold layer which reflects radiation at $10.6 \mu \mathrm{m}$ in order to get more energy into the cell and to avoid additional heating of the bottom plate and the bottom plate heater.

One also had to re-machine several parts of the experiment. In order to work with as little $\mathrm{CS}_{2}$ as possible the vessel with the bellows was disconnected from the system and a new smaller bellows was installed directly into the main pressure vessel. In this way one reduces the amount of $\mathrm{CS}_{2}$ inside the system by more than one liter. Due to the low flash point of $\mathrm{CS}_{2}\left(-30^{\circ} \mathrm{C}\right)$, filling the apparatus is done in a closed system as shown in Fig. 6.6. The sketch is very similar to Fig. 3.1. The pressure regulation vessel is drawn smaller than in Fig. 3.1 and the pressure equilibration vessel does not exist anymore, since the bellows is included into the main vessel. Therefore, one has another glass bottle, located under a chemical hood which is used as a $\mathrm{CS}_{2}$ reservoir. In order to have as little oxygen as possible in the system, a steady flow of nitrogen is introduced into the system. For pumping the $\mathrm{CS}_{2}$ from the reservoir into the main vessel, a peristaltic pump (Medorex, TV/50) is used. In this way the pump does not get in contact with the cooling liquid, but just massages the tube and pushes the fluid in this way in the chosen direction. The part of the tube close to the pump is made out of Viton ${ }^{\circledR}$ while other parts are made out of Teflon. It is worth mentioning that Viton ${ }^{\circledR}$ shows a good compatibility with $\mathrm{CS}_{2}$, while Viton-Extreme ${ }^{\circledR}$ is even more compatible with $\mathrm{CS}_{2}{ }^{3}$. That is why all the o-rings in the experiment were changed to Viton-Extreme ${ }^{\circledR}$. In order to remove as much $\mathrm{CS}_{2}$ vapor as possible from the main vessel and the inner tube, the $\mathrm{CS}_{2}$ reservoir bottle is cooled by dry $\mathrm{CO}_{2}$ ice in order to minimize the vapor pressure in the bottle and thus the vapor condensates out in the reservoir. The nitrogen reservoir is connected via a pressure reducer to the tubing. The reducer should always be set to values lower

${ }^{3}$ Information about compatibility can be found here:
http://flw.com/datatools/compatibility/


than 2 bar. In order to avoid to high pressure in the $\mathrm{CS}_{2}$ bottle, a safety valve at the bottle contains of a simple rubber plug (red piece in Fig. 6.6) which will jump out at the slightest over pressure in the bottle.

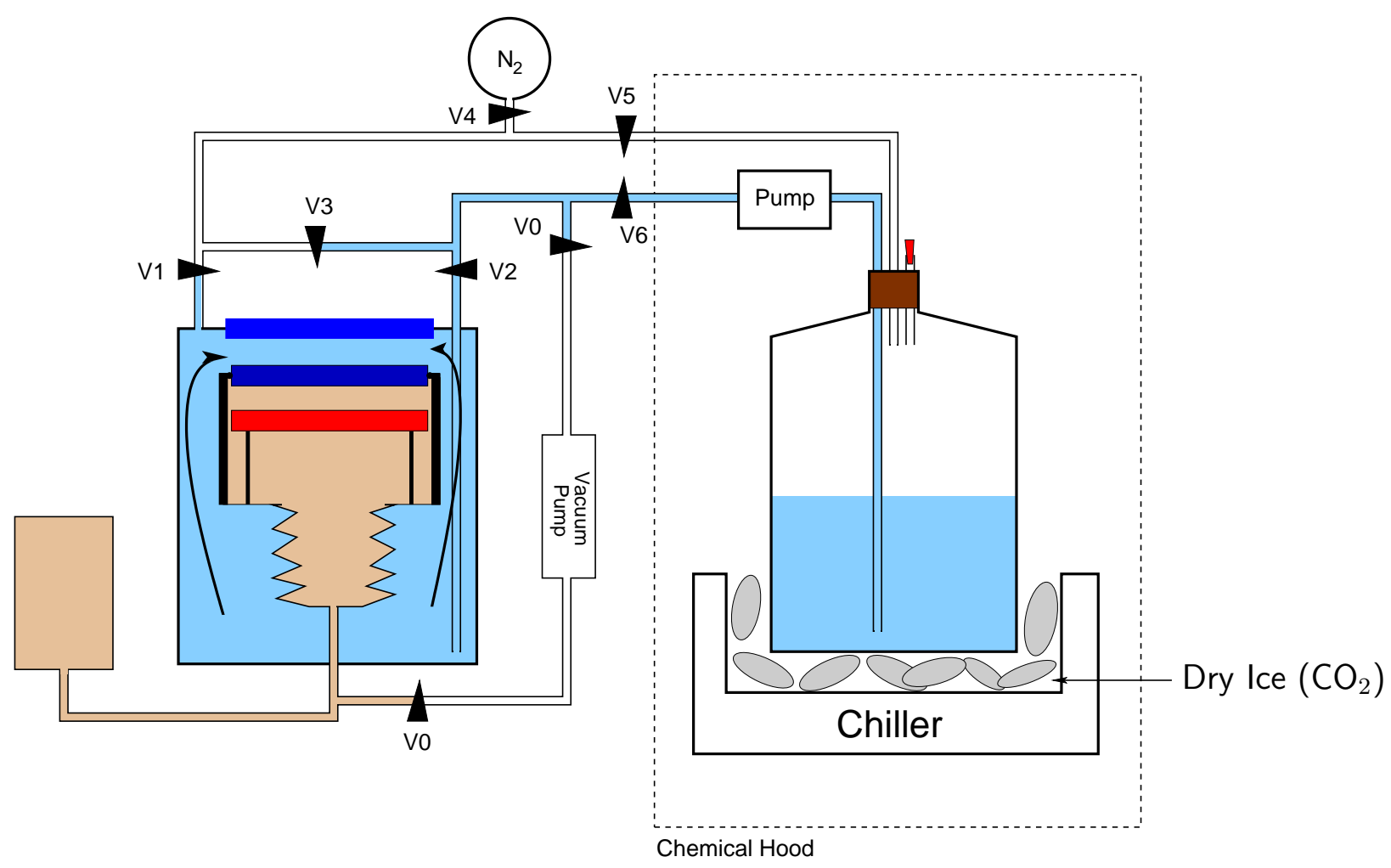

Figure 6.6: Schematic drawing of the $\mathrm{CS}_{2}$ filling system

Specific care has to be taken during filling and emptying the experimental apparatus. A complete procedure is given in the appendix. 


\section{Chapter 7}

\section{Summary}

Pattern formation in physics, chemistry and biology has been intensively investigated in the last century [31, 2, 109]. In order to understand the basic pattern forming mechanisms, simplified isotropic and homogeneous model systems were studied. By contrast to these model systems, pattern formation in nature is usually affected by anisotropies and inhomogeneities. In order to investigate these effects in laboratory experiments, one can externally force the otherwise homogeneous system, by modulating system parameters in space (spatial forcing) or in time (temporal forcing).

While the effect of temporal forcing on pattern forming systems was studied extensively in the past $([110,59,62,58])$, work on spatial forcing is rare $[67,65$, $96,69]$. In contrast to temporal forcing, where the system can only respond in one dimension, with spatial forcing the system can respond and build resonances in two or three dimensions. Here, we apply spatial forcing on a large aspect ratio thermal convection system. We realize forcing by a quasi one-dimensional periodic stripe pattern that was imprinted on the bottom plate of the convection cell via photo lithography. We conduct two sets of experiments.

First, the one-dimensional periodic forcing was combined with an anisotropy caused by inclination (Chap. 4). Due to inclination, a large scale shear flow is created, that has a strong symmetry breaking effect on the occurring pattern. In an unforced system, for small inclination angles $\left(\gamma<\gamma_{c d}\right)$, the occurring convection rolls are aligned with their axis in the direction of gravity, and for larger inclination angles $\left(\gamma_{c d}<\gamma\right)$ the occurring rolls - now driven by a shear instability - are aligned perpendicular to gravity. In the forced inclined layer convection system the resulting pattern therefore depend strongly on the angle $\varphi$, which is the angle between forcing stripes and gravity.

If longitudinal rolls are forced $(\varphi=0)$ and small and moderate inclination angles are considered $\left(\gamma<\gamma_{c d}\right)$, forcing has a stabilizing effect. Forced rolls are stable up to higher $\varepsilon$ than in the unforced case, and patterns above the instability line 
of forced rolls are less dynamic and have fewer defects than in the unforced case. In general, we observe for a forcing with an angle of $\varphi=0$ most of the pattern that are also found in the unforced system.

In experiments with different angles $\left(\varphi=90^{\circ}\right.$ and $\left.\varphi=60^{\circ}\right)$, the gravity and the boundary forcing act in different directions. For inclination angles smaller than $78^{\circ}$, the resulting large-scale shear flow forces straight rolls to be aligned parallel to gravity, but the boundary forcing leads to rolls aligned with an angle $\varphi$ to gravity. By changing $\varepsilon$, one can tune the relative strength of both mechanisms. The system responds with patterns that utilize inherent modes from the unforced system and the forcing mode. Thus, we observe multiple new patterns. For large inclination angles we find superlattice patterns, such as Scepter, Heart and Pretzel patterns.

In the second set of experiments, forcing takes place in a horizontally aligned convection cell and we vary the forcing wave vector (Chap. 5). Especially of interest is the question on how the system reacts if the forcing wave numbers were located outside the Busse balloon for unforced Rayleigh-Bénard convection. Here, for example, Varicose or Kink patterns occur as a modified version of the well known Skewed Varicose instability for unforced RBC. For forcing with different wave numbers, a theory exists $([98,72])$ which exhibits qualitative similarities to the experimental results, but which does not agree quantitatively.

In addition to presenting experiments we provide in Chapter 6 an outlook on experiments on optical forcing, where heat energy is introduced into the system by absorption of IR light. This way of spatial forcing promises several advantages in comparison to boundary forcing as performed here. In such experiments, one is able to change the forcing pattern easily and can also force dynamic patterns. In addition, the shadowgraph shows the correct temperature distribution in the convection cell and is not influenced by the forcing itself, as it is in the case of boundary forcing. Therefore, spatial forcing with IR light will offer an experimental approach, which allows to gain a deeper insight into the effect of spatio-temporal forcing on an otherwise isotropic pattern forming system.

The forcing of a pattern forming system is a useful means to study pattern forming mechanisms (i), to determine stability regions (ii) and to investigate their instability boundaries (iii). With the help of forcing one can also control the system up to a certain degree. In this way, one can reduce or suppress spatiotemporal chaos and stabilize non-linear systems if a chaotic behavior is unwanted. An example is the heart beat, where coherent excitation waves break and form chaotic spirals, leading to a strongly reduced pumping performance [111]. Similar examples include avoiding fatal voltage collapse in power networks [112] or chaos in the brain [113] and other neural networks. In most of these cases temporal forcing is used in order to stabilize an chaotic system.

The stabilizing effect of periodic spatial forcing was demonstrated in this thesis. 
Examples include the shift of the instability line of longitudinal rolls to higher control parameter $\varepsilon$ for longitudinal forcing $(\varphi=0)$, the reduced disorder in undulation patterns, or the stabilization of transverse bursts into scepter pattern with a reduced dynamic.

An application for spatial forcing of a pattern forming system was suggested by Manor, Hagberg and Meron [114], to optimize the bio-productivity in arid and semi-arid regions. In these regions, where the growth of vegetation is limited by the amount of rain, vegetation stripes occur naturally on hill slopes along horizontal contour lines (Fig. 7.1). For agriculture or vegetation recovery ditches are built along the contour lines in order to harvest rain water. Along these ditches vegetation is planted and grows well due to the higher soil-water density. These ditches can be seen as a forcing of a stripe pattern similar to the boundary forcing presented in this thesis.
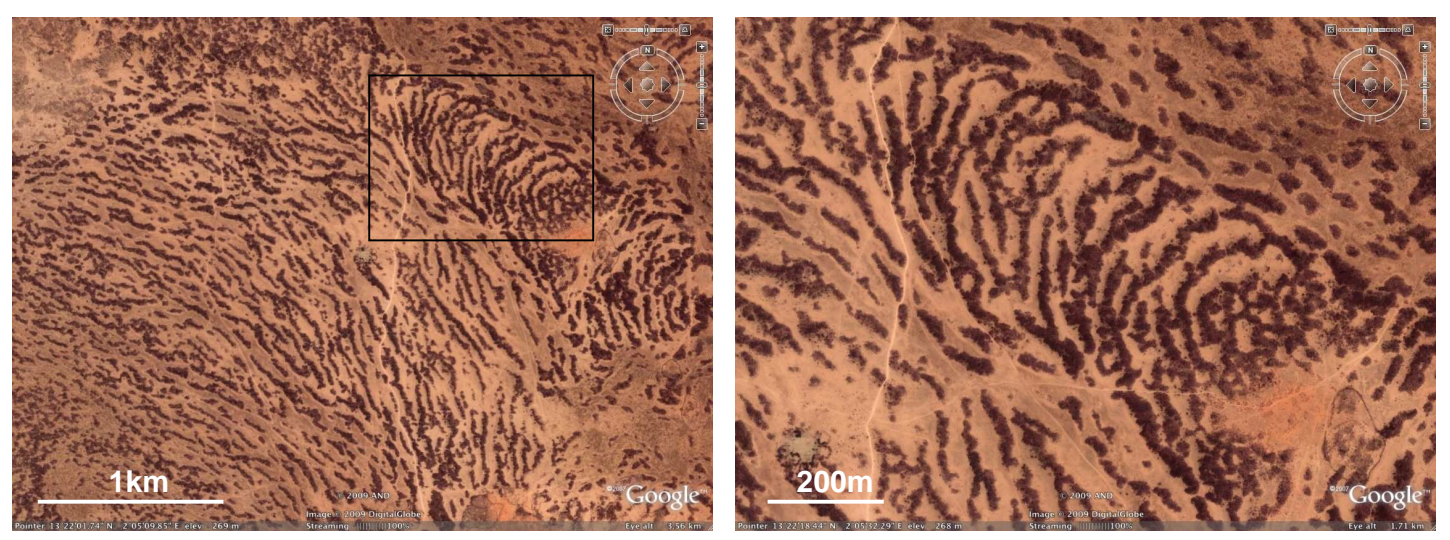

Figure 7.1: Banded vegetation in an arid region. The right picture shows the marked rectangular section of the left one. Pictures show an area close to Niamey (Niger). (Images from GoogleEarth)

Pattern forming systems in general and with forcing in particular are often highly non-linear systems and provide with their complexity an interesting field of research. There are still interesting questions to answer in this field. These include experiments on convection due to internal heating or pattern formation in a spherical symmetry. In both cases, simulation were done in the past, but the experimental verification is still missing. 


\section{Appendix A}

\section{Boussinesq Equations}

\section{A.1 Unforced ILC}

In order to derive the Boussinesq equation for the inclined layer convection one has to take into consideration the existence of a mean flow $\vec{U}_{0}$ as shown in Eq. 2.34. But again, the mean flow itself is influenced by the onset of convection and therefore following ansatz has to be made:

$$
\vec{v}=U_{0} \hat{y}+\vec{U}_{1}+\vec{u}
$$

Where $\vec{U}_{1}$ is the modulation of the mean flow due to convection.

Therefore the Boussinesq equations for the fields $\theta, g, f$, and $U_{1}$ for inclined layer convection (ILC) become:

The $f$-equation:

$$
\begin{aligned}
\nabla^{4} \Delta_{2} f+\tan (\gamma) \partial_{y z}^{2} \theta-\Delta_{2} \theta= & \operatorname{Pr}^{-1}[\hat{\delta} \cdot((\hat{\delta} f+\hat{\epsilon} g) \cdot \nabla(\hat{\delta} f+\hat{\varepsilon} g)) \\
& +\left(U_{1 x} \partial_{x}+U_{1 y} \partial_{y}\right) \nabla^{2} \Delta_{2} f \\
& -\left(\partial_{z z}^{2} U_{1 x} \partial_{x}+\partial_{z z}^{2} U_{1 y} \partial_{y}\right) \Delta_{2} f \\
& +\left(U_{0} \partial_{y}+\partial_{t}\right) \nabla^{2} \Delta_{2} f \\
& \left.-\partial_{z z}^{2} U_{0} \partial_{y} \Delta_{2} f\right]
\end{aligned}
$$


The $g$-equation:

$$
\begin{aligned}
\nabla^{2} \Delta_{2} g+\tan (\gamma) \partial_{x} \theta= & \operatorname{Pr}^{-1}[\hat{\epsilon}((\hat{\delta} f+\hat{\epsilon} g) \cdot \nabla(\hat{\delta} f+\hat{\epsilon} g)) \\
& +\left(U_{1 x} \partial_{x}+U_{1 y} \partial_{y}\right) \Delta_{2} g \\
& -\left(\partial_{z} U_{1 y} \partial_{x}-\partial_{z} U_{1 x} \partial_{y}\right) \Delta_{2} f \\
& +\left(U_{0} \partial_{y}+\partial_{t}\right) \Delta_{2} g \\
& \left.-\left(\partial z U_{0} \partial_{x}\right) \Delta_{2} f\right] .
\end{aligned}
$$

The $\theta$-equation:

$$
\begin{aligned}
\nabla^{2} \theta-R a \Delta_{2} f= & (\hat{\delta} f+\hat{\epsilon} g) \cdot \nabla \theta+\left(U_{1 x} \partial_{x}+U_{1 y} \partial_{y}\right) \theta \\
& +\left(U_{0} \partial_{y}+\partial_{t}\right) \theta
\end{aligned}
$$

In addition one has two equations for both the $\mathrm{x}$ and $\mathrm{y}$ components of $U_{1}$ :

$$
\begin{aligned}
\left(\partial_{z z}^{2}-\operatorname{Pr}^{-1} \partial_{t}\right) U_{1 x}+\partial_{z} \operatorname{Pr}^{-1} \overline{\left[\Delta_{2} f\left(\partial_{x z}^{2} f-\partial_{y} g\right)\right]} & =0 \\
\left(\partial_{z z}^{2}-\operatorname{Pr}^{-1} \partial_{t}\right) U_{1 y}+\partial_{z} P^{-1} \overline{\left[\Delta_{2} f\left(\partial_{y z}^{2} f+\partial_{x} g\right)\right]}+\bar{\theta} \tan (\gamma) & =0 .
\end{aligned}
$$

The line above the second term in the equations above stand for the average over both horizontal directions. In the same way, $\bar{\theta}$ is the horizontal average of $\theta$.

\section{A.2 Forced ILC:}

For forced ILC, one just has to substitute all occurence of $\theta$ in the equations above by $\theta+T_{\text {mod }}$. With $T_{\text {mod }}$ given in Eq. 2.13 one gets the Boussinesq equations for the forced ILC:

The equation $f$ :

$$
\begin{aligned}
\nabla^{4} \Delta_{2} f+\tan (\gamma) \partial_{y z}^{2}\left(\theta+T_{\text {mod }}\right)-\Delta_{2}\left(\theta+T_{\text {mod }}\right)= & \operatorname{Pr}^{-1}[\hat{\delta} \cdot((\hat{\delta} f+\hat{\epsilon} g) \cdot \nabla(\hat{\delta} f+\hat{\varepsilon} g)) \\
& +\left(U_{1 x} \partial_{x}+U_{1 y} \partial_{y}\right) \nabla^{2} \Delta_{2} f \\
& -\left(\partial_{z z}^{2} U_{1 x} \partial_{x}+\partial_{z z}^{2} U_{1 y} \partial_{y}\right) \Delta_{2} f \\
& +\left(U_{0} \partial_{y}+\partial_{t}\right) \nabla^{2} \Delta_{2} f \\
& \left.-\partial_{z z}^{2} U_{0} \partial_{y} \Delta_{2} f\right] .
\end{aligned}
$$


The equation for $g$ :

$$
\begin{aligned}
\nabla^{2} \Delta_{2} g+\tan (\gamma) \partial_{x}\left(\theta+T_{\text {mod }}\right)= & \operatorname{Pr}^{-1}[\hat{\epsilon}((\hat{\delta} f+\hat{\epsilon} g) \cdot \nabla(\hat{\delta} f+\hat{\epsilon} g)) \\
& +\left(U_{1 x} \partial_{x}+U_{1 y} \partial_{y}\right) \Delta_{2} g \\
& -\left(\partial_{z} U_{1 y} \partial_{x}-\partial_{z} U_{1 x} \partial_{y}\right) \Delta_{2} f \\
& +\left(U_{0} \partial_{y}+\partial_{t}\right) \Delta_{2} g \\
& \left.-\left(\partial z U_{0} \partial_{x}\right) \Delta_{2} f\right] .
\end{aligned}
$$

The equation for $\theta$ :

$$
\begin{aligned}
\nabla^{2}\left(\theta+T_{\text {mod }}\right)-R a \Delta_{2} f= & (\hat{\delta} f+\hat{\epsilon} g) \cdot \nabla\left(\theta+T_{\text {mod }}\right)+\left(U_{1 x} \partial_{x}\right. \\
& \left.+U_{1 y} \partial_{y}\right)\left(\theta+T_{\text {mod }}\right) \\
& +\left(U_{0} \partial_{y}+\partial_{t}\right)\left(\theta+T_{\text {mod }}\right) .
\end{aligned}
$$

The equations for $\vec{U}_{1}$ :

$$
\begin{aligned}
& \left(\partial_{z z}^{2}-\operatorname{Pr}^{-1} \partial_{t}\right) U_{1 x}+\partial_{z} \operatorname{Pr}^{-1} \overline{\left[\Delta_{2} f\left(\partial_{x z}^{2} f-\partial_{y} g\right)\right]} \\
& \quad=0 \\
& \left(\partial_{z z}^{2}-\operatorname{Pr}^{-1} \partial_{t}\right) U_{1 y}+\partial_{z} P^{-1} \overline{\left[\Delta_{2} f\left(\partial_{y z}^{2} f+\partial_{x} g\right)\right]}+\overline{\left(\theta+T_{\text {mod }}\right)} \tan (\gamma) \\
& \quad=0 .
\end{aligned}
$$




\section{Appendix B}

\section{Filling and emptying procedure for $\mathrm{CS}_{2}$}

Following procedure should provide a maximal safety during filling and emptying the apparatus. A maximum of attention and care has to be taken since $\mathrm{CS}_{2}$ is highly flammable, poisonous and volatile. The valves correspond with their label in Fig. 6.6.

Filling:

- All valves have to be closed and the nitrogen pressure reducer has to be set to a pressure lower than 1.5 bar

- Open V2 and V0 and evacuate the working gas chamber and the cooling liquid chamber at the same time. Take care that the pressure difference between the two chambers is always lower than 2 psi. A beep indicates a critical pressure difference.

- Close V0 if both chambers are evacuated

- Open V4 and V5 so that nitrogen fills the $\mathrm{CS}_{2}$ bottle and parts of the pipe

- Open slowly V1 and fill nitrogen into the system. At the same time you should fill the working gas chamber with $\mathrm{CO}_{2}$. Increase the pressure in both chambers slowly until $1 \mathrm{~atm}$ is reached and listen carefully to a beep, indicating that the pressure difference between both chambers reaches a critical value.

- If a pressure of $1 \mathrm{~atm}$ (16 psi) is reached, stop the filling of $\mathrm{CO}_{2}$, open V5 and V6 and start peristaltic pump in order to pump $\mathrm{CS}_{2}$ into the main vessel. 
- Filling procedure may take up to 2 hours. The chamber has to be filled completely with $\mathrm{CS}_{2}$ as shown in the sketch in Fig. 6.6. Try to avoid bubbles by slightly turning the whole system.

- If main vessel is filled, switch off the pump.

- Close V1 and V2 and open V3.

- Start pump in the other direction - pump $\mathrm{CS}_{2}$ from the tubes into the reservoir bottle for at least one hour.

- Close V4 and close the $\mathrm{CS}_{2}$ bottle with the (red) plug, in case it opened during the filling procedure.

- Switch off pump, close V5 and V6 and continue filling $\mathrm{CO}_{2}$ into the system until desirable pressure is reached.

Emptying:

- Set $\mathrm{N}_{2}$ pressure to lower than 1.5 bar.

- Fill dry ice $\left(\mathrm{CO}_{2}\right)$ to the chiller in order to cool down the $\mathrm{CS}_{2}$ reservoir.

- Empty $\mathrm{CO}_{2}$ side until 1 atm is reached.

- Open V1, V2, V4, V5, and V6 and close V3.

- Start pump and pump $\mathrm{CS}_{2}$ from the apparatus to the reservoir for at least one hour.

- Close all valves and close the third orifice of the $\mathrm{CS}_{2}$ bottle with the (red) plug.

In this way the apparatus can be filled and emptied in a save way. Beside some nitrogen and $\mathrm{CS}_{2}$ vapor which evaporates in the chemical hood, the system is completely closed and $\mathrm{CS}_{2}$ can not enter the room. 


\section{Appendix C}

\section{Suplementary Material on CD-ROM}

\section{C.1 Movies}

Enclosed to the thesis movies and other suplementary material can be found on a CD-ROM. All movies are compressed with DIVX. When the direction of the gravity is not mentioned explicitly, all movies are aligned with the direction of gravity in the experiment. That means, that the gravity always points from up to down in each movie.

Pattern for longitudinal forcing $(\varphi=0)$ :

UN.avi:

Undulations at $\gamma=40^{\circ}$ and $\varepsilon=0.315$ (movie is digitally enhanced)

CR0.avi: $\quad$ Crawling Rolls at $\gamma=40^{\circ}$ and $\varepsilon=0.72$

TB.avi: $\quad$ Transverse Bursts at $\gamma=80^{\circ}$ and $\varepsilon=0.17$

LB.avi: $\quad$ Longitudinal Bursts at $\gamma=85^{\circ}$ and $\varepsilon=0.185$

STChaos.avi: $\quad$ Spatio-temporal chaos at $\gamma=0^{\circ}$ and $\varepsilon=2.65$ ).

Kink.avi: $\quad$ Coherent kink lines at $\gamma=5^{\circ}$ and $\varepsilon=2.345$

VaricoseDrift.avi: Drifting varicose pattern (at $\gamma=10^{\circ}$ and $\varepsilon=2.4$ )

LocalKink.avi: Localized crawling kinks embedded in an otherwise steady roll pattern at $\theta=10^{\circ}$ and $\varepsilon=1.719$ due to a reduction of $\varepsilon$ from a chaotic state

Patterns due to transverse forcing $\left(\varphi=90^{\circ}\right)$ :

CR90.avi: Crawling Rolls at $\gamma=30^{\circ}$ and $\varepsilon=0.69$

BM.avi: Bimodal Patter at $\gamma=60^{\circ}$ and $\varepsilon=0.20$ (movie was taken with lower camera resolution as in Fig. 4.29)

SP.avi: $\quad$ Scepter Pattern at $\gamma=72.5^{\circ}$ and $\varepsilon=0.321$

HP.avi: Heart Pattern at $\gamma=90^{\circ}$ and $\varepsilon=0.039$ 
Patterns in horizontal convection due to a variation of $q_{f} / q_{c}$ :

BrickWall.avi: Brick Wall pattern at $q_{f}=0.68 q_{c}$ and $\varepsilon=0.28$

\section{C.2 RBC - Program to calculate fluid proper- ties}

The program, to calculate the fluid properties of several fluids was developed in G. Ahlers lab at the UCSB in Santa Barbara and can be found on the CD-ROM in the RBC-folder. The program is written in $\mathrm{C}$ language and does not need any specific external libraries. A Makefile exist for easy compilation. Please read README for further information. 


\section{Bibliography}

[1] D. Aubin, The memory of life itself: Bénard's cells and the cinematography of self-organization, Studies in History and Philosophy of Science 39, 359 (2008).

[2] A. M. Turing, The chemical basis of morphogenesis, Philosophical Transactions of the Royal Society of London, Series B, Biological Sciences 237, 37 (1952).

[3] J. D. Watson and F. Crick, A structure for deoxyribose nucleic acid, Nature 171, 737 (1953).

[4] V. Castets, E. Dulos, J. Boissonade, and P. D. Kepper, Experimental evidence of a sustained turing-type nonequilibrium chemical pattern, Physical Review Letters 64, 2953 (1990).

[5] J. D. Murray, Mathematical Biology (Springer-Verlag, 1989).

[6] D. G. Míguez, E. M. Nicola, A. P. M. nuzuri, J. Casademunt, F. Sagués, and L. Kramer, Traveling-stripe forcing generates hexagonal patterns, Physical Review Letters 93, 048303 (2004).

[7] L. Yang, M. Dolnik, A. M. Zhabotinsky, and I. R. Epstein, Turing patterns beyond hexagons and stripes, Chaos 16, 037114 (2006).

[8] M. Dolnik, I. Berenstein, A. M. Zhabotinsky, and I. R. Epstein, Spatial periodic forcing of turing structures, Physical Review Letters 87, 23803 (2001).

[9] J. D. Murray, On pattern formation mechanisms for lepidopteran wing patterns and mammalian coat markings, Phil. Trans. R. Soc. Lond. B 295, 473 (1981).

[10] T. Miura, K. Shiota, G. Morriss-Kay, and P. K. Maini, Mixed-mode pattern in doublefoot mutant mouse limb - turing reaction-diffusion model on a growing domain during limb development, Journal of Theoretical Biology 240, 562 (2006). 
[11] T. Andl, S. T. Reddy, T. Gaddapara, and S. E. Millar, Wnt signals are required for the initiation of hair follicle development, Development Cell $\mathbf{2}$, $643(2002)$.

[12] A. Niida, T. Hiroko, M. Kasai, Y. Furukawa, Y. Nakamura, Y. Suzuki, S. Sugano, and T. Akiyama, DKK1, a negative regulator of Wnt signaling, is a target of the $\beta$-catenin/TCF pathway, Oncogene 23, 8520 (2004).

[13] S. Sick, S. Reinker, J. Timmer, and T. Schlake, WNT and DKK determine hair follicle spacing through a reaction-diffusion mechanism, Science 314, 1447 (2007).

[14] Nature's Pattern Makers, MaxPlanckResearch (3/2008), by C. Beck.

[15] M. C. Cross and P. C. Hohenberg, Pattern formation outside equilibrium, Review of Modern Physics 65, 851 (1993).

[16] P. Manneville, Dissipative Structures and Weak Turbulence (Academic Press, INC., 1990).

[17] H. Haken, Synergetics of brain function, International Journal of Psychophysiology 60, 110 (2006), ISSN 0167-8760, models and Theories of Brain Function with Special Emphasis on Cognitive Processing.

[18] W. Brock and A. Xepapadeas, Pattern formation, spatial externalities and regulation in coupled economic-ecological systems, MPRA Paper 9105, University Library of Munich, Germany (2008), URL http://ideas.repec.org/p/pra/mprapa/9105.html.

[19] M. Cross and H. Greenside, Pattern Formation and Dynamics in Nonequilibrium Systems (Cambridge University Press, 2009).

[20] B. Diu, C. Guthmann, D. Lederer, and B. Roulet, Grundlagen der Statistischen Physik (Walter de Gruyter, 1994).

[21] M. D. Cowley and R. E. Rosensweig, The interfacial stability of a ferromagnetic fluid, Journal of Fluid Mechanics 30, 671 (1967).

[22] I. Hamley, The Physics of Block Copolymers (Oxford Science Publication, 1999).

[23] J. Swift and P. C. Hohenberg, Hydrodynamic fluctuations at the convective instability, Physical Review A 15, 319 (1977).

[24] R. Hoyle, Pattern Formation - An introduction to methods (Cambridge University Press, 2006). 
[25] L. A. Segel, Distant side-walls cause slow amplitude modulation of cellular convection, Journal of Fluid Mechanics 38, 203 (1969).

[26] A. C. Newell and J. A. Whitehead, Finite bandwith, finite amplitude convection, Journal of Fluid Mechanics 38, 279 (1969).

[27] F. H. Busse, A model of the geodynamo, Geophys. J. R. astr. Soc. 42 (1975).

[28] G. A. Glatzmeier and P. H. Roberts, A three-dimensional self-consistent computer simulation of a geomagnetic field reversal, Nature 377, 203 (1995).

[29] D. L. Turcotte and E. R. Oxburg, Finite amplitude convective cells and continental drift, Journal of Fluid Mechanics 28, 29 (1967).

[30] E. Bodenschatz, W. Pesch, and G. Ahlers, Recent developments in RayleighBénard convection, Annu. Rev. Fluid Mech. 32, 709 (2000).

[31] H. Bénard, Les tourbillon cellulaire dans une nappe liquide, Rev. Gén. Sci. pures et apll. 11, 1261 (1900).

[32] J. R. A. Pearson, On convection cells induced by surface tension, Journal of Fluid Mechanics Digital Archive 4, 489 (1958).

[33] F. H. Busse, Non-linear properties of thermal convection, Rep. Prog. Phys. 41, 1929 (1978).

[34] F. H. Busse and R. M. Clever, Instabilities of convection rolls in a fluid of moderate prandtl number, J. Fluid Mech. 91, 319 (1979).

[35] F. H. Busse and J. A. Whitehead, Oscillatory instabilities in large prandtl number convection, J. Fluid Mech. 66, 67 (1974).

[36] F. H. Busse and J. A. Whitehead, Instabilities of convection rolls in a high Prandtl number fluid, Journal of Fluid Mechanics 47, 305 (1971).

[37] R. V. Cakmur, D. A. Egolf, B. B. Plapp, and E. Bodenschatz, Bistability and competition of spatiotemporal chaotic and fixed point attractors in rayleigh-bénard convection, Phys. Rev. Lett. 79, 1853 (1997).

[38] M. M. Chen and J. A. Whitehead, Evolution of two-dimensional periodic Rayleigh convection cells of arbitrary wave-numbers, J. Fluid Mech. 31, 1 (1968).

[39] W. Tian, D. J. Parker, and C. A. D. Kilburn, Observation and numerical simulation of atmospheric cellular convection over mesoscale topography, Monthly Weather Review 131, 222 (2003). 
[40] M. Kuecken and A. C. Newell, Fingerprint formation, Journal of Theoretical Biology 235, 71 (2005).

[41] E. Venturino and A. B. Medvinsky, The role of periodic boundary forcing in plankton pattern formation, Ecological Modelling 140, 255 (2001).

[42] C. Groh, R. Richter, I. Rehberg, and F. H. Busse, Reorientation of hexagonal pattern under broken symmetry: hexagon-flip, Phys. Rev. E. 76, 055301 (2007).

[43] M. Bär, E. Meron, and C. Utzny, Pattern formation on anisotropic and heterogeneous catalytic surfaces, Chaos 12, 204 (2002).

[44] M. A. Kessler and B. T. Werner, Self-organization of sorted patterned ground, Science 299, 380 (2003).

[45] W. Brunner, J. McCoy, W. Pesch, and E. Bodenschatz, Hexaroll chaos in inclined layer convection (2006), URL http://www . citebase .org/abstract?id=oai : arXiv . org: nlin/0601016.

[46] U. H. Kurzweg, Stability of natural convection within an inclined channel, Journal of Heat Transfer 14, 190 (1970).

[47] R. M. Clever and F. H. Busse, Instabilities of longitudinal rolls in an inclined layer, J. Fluid Mech. 81, 107 (1977).

[48] K. Daniels, B. Plapp, and E. Bodenschatz, Pattern formation in inclined layer convection, Physical Review Letters 84, 5320 (2000).

[49] K. E. Daniels, Pattern formation and dynamics in inclined layer convection, Ph.D. thesis, Cornell University (2002).

[50] K. Daniels, O. Brausch, W. Pesch, and E. Bodenschatz, Competition and bistability of ordered undulations and undulation chaos in inclined layer convection, Journal of Fluid Mechanics 497, 261 (2008).

[51] O. Brausch, Rayleigh-Bénard Konvektion in verschiedenen isotropen und anisotropen Systemen, Ph.D. thesis, Univsersität Bayreuth (2001).

[52] S. Chandrasekhar, Hydrodynamic and Hydromagnetic Stability (Dover Publications, Inc., 1961).

[53] K. E. Daniels, R. J. Wiener, and E. Bodenschatz, Localized transverse bursts in inclined layer convection, Physical Review Letters 91, 114501 (2003).

[54] R. Chacon, Melnikov method approach to control of homoclinic/heteroclinic chaos by weak harmonic excitations, Phil. Trans. R. Soc. Lond. A 15 (2006). 
[55] J. Kim, J. Lee, and B. Kahng, Responses of the complex ginzburg-landau equation under harmonic forcing, Physica A: Statistical Mechanics and its Applications 315, 330 (2002), ISSN 0378-4371.

[56] P. Parmananda, M. Rivera, B. Green, and J. Hudson, Controlling complexity using forcing: simulations and experiments, Applied Mathematics and Computation 164, 467 (2005), 12th International Workshop on Dynamics and Control.

[57] A. L. Lin, A. Hagberg, A. Ardelea, M. Bertram, H. L. Swinney, and E. Meron, Four-phase patterns in forced oscillatory systems, Physical Review E 62, 3790 (2000).

[58] S. Alonso, F. Sagues, and A. S. Mikhailov, Taming Winfree Turbulence of Scroll Waves in Excitable Media, Science 299, 1722 (2003).

[59] C. W. Meyer, D. S. Cannell, and G. Ahlers, Hexagonal and roll flow patterns in temporally modulated rayleigh-bénard convection, Phys. Rev. A 45, 8583 (1992).

[60] P. C. Hohenberg and J. B. Swift, Hexagons and rolls in periodically modulated rayleigh-bénard convection, Phys. Rev. A 35, 3855 (1987).

[61] J. L. Rogers, W. Pesch, O. Brausch, and M. F. Schatz, Complex-ordered patterns in shaken convection, Phys. Rev. E 71, 066214 (2005).

[62] J. L. Rogers, M. F. Schatz, J. L. Bougie, and J. B. Swift, Rayleigh-bénard convection in a vertically oscillated fluid layer, Phys. Rev. Lett. 84, 87 (2000).

[63] J. L. Rogers, M. F. Schatz, O. Brausch, and W. Pesch, Superlattice patterns in vertically oscillated rayleigh-bénard convection, Phys. Rev. Lett. 85, 4281 (2000).

[64] M. Lowe, J. P. Gollub, and T. C. Lubensky, Commensurate and incommensurate structures in a nonequilibrium system, Physical Review Letters 51, 786 (1983).

[65] M. Lowe and J. P. Gollub, Solitons and the commensurate- incommensurate transition in a convecting nematic fluid, Physical Review A 31, 3893 (1985).

[66] P. Coullet, Commensurate-incommensurate transition in nonequilibrium system, Physical Review Letters 56 (1986).

[67] R. E. Kelly and D. Pal, Thermal convection with spatially periodic boundary conditions: resonant wavelength excitation, J. Fluid Mech. 86, 433 (1978). 
[68] J. H. McCoy, Adventures in pattern formation: Spatially periodic forcing and self-organization, Ph.D. thesis, Cornell University (2007).

[69] J. McCoy, W. Brunner, W. Pesch, and E. Bodenschatz, Johns prl, Physical Review Letters 101, 254102 (2008).

[70] F. H. Busse, The stability of finite amplitude cellular convection and its relation to an extremum principle, Journal of Fluid Mechanics 30, 625 (1967).

[71] E. A. Spiegel and G. Veronis, On the Boussinesq approximation for a compressible fluid, Astrophys. J. 131, 442 (1960).

[72] W. Pesch, Convection with Modulation (2005), Technical Report (unpublished).

[73] G. Freund, Über den Einfluss einer räumlichen Temperaturmodulation auf die Rayleigh-Bénard Konvektion (2007).

[74] J. R. de Bruyn, E. Bodenschatz, S. W. Morris, S. P. Trainoff, Z. Hu, D. S. Cannell, and G. Ahlers, Apparatus for study of Rayleigh-Bénard convection in gases under pressure, Rev. Sci. Instrum. 67, 2043 (1996).

[75] B. B. Plapp, Spiral pattern formation in Rayleigh-Bénard convection, Ph.D. thesis, Cornell University (1997).

[76] Handbook of Chemistry and Physics (Taylor and Francis Group, LLC, 2009), URL http://www.hbcpnetbase.com/.

[77] G. Ahlers, Computer program to calculate material properties for different fluids (can be found on attended CD-ROM).

[78] MicroChem webpage, URL www.microchem.com.

[79] EV 620 Users Manual, EVG (2000).

[80] G. S. Settles, Schlieren and Shadowgraph Techniques. Visualizing Phenomena in Transparent Media (Springer, 2001).

[81] S. Rasenat, G.Hartung, B. L. Winkler, and I. Rehberg, The shadowgraph method in convection experiments, Experiments in Fluids 7, 412 (1989).

[82] G. Ahlers, Computer program to calculate material properties for different fluids.

[83] S. P. Trainoff and D. S. Cannell, Physical optics treatment of the shadowgraph, Physics of Fluids 14, 1340 (2002). 
[84] M. V. Berry and E. Bodenschatz, Caustics, multiply reconstructed by talbot interference, Journal of Modern Optics 46, 349 (1999).

[85] A. Borreman et al., in Proceedings Symposium IEEE (2002).

[86] URL http://rsbweb.nih.gov/ij/.

[87] G. Seiden, S. Weiss, J. McCoy, W. Pesch, and E. Bodenschatz, Pattern forming system in the presence of different symmetry-breaking mechanisms, Physical Review Letters 101, 214503 (2008).

[88] J. Wesfreid, Y. Pomeau, M. Dubois, C. Normand, and P. Berge, Critical effects in rayleigh-benard convection, J. Phys. France 39, 725 (1978).

[89] R. Heinrichs, G. Ahlers, and D. S. Cannell, Traveling waves and spatial variation in the convection of a binary mixture, Phys. Rev. A 35, 2761 (1987).

[90] R. Richter and I. V. Barashenkov, Two-dimensional solitons on the surface of magnetic fluids, Phys. Rev. Lett. 94, 184503 (2005).

[91] M. Auer, F. H. Busse, and R. M. Clever, Three-dimensional convection driven by centrifugal buoyancy, Journal of Fluid Mechanics 301, 371 (1995).

[92] M. Jaletzke and F. H. Busse, New pattern of centrifugally driven thermal convection, PNAS 97, 5060 (2000).

[93] A. Kudrolli, B. Pier, and J. P. Gollub, Superlattice patterns in surface waves, Physica D 123, 99 (1998).

[94] E. Große Westhoff, R. Herrero, T. Ackemann, and W. Lange, Self-organized superlattice patterns with two slightly differing wave numbers, Phys. Rev. E 67, 025203 (2003).

[95] S. L. Judd and M. Silber, Simple and superlattice turing patterns in reaction-diffusion systems: Bifurcation, bistability, and parameter collapse, Physica D 136, 45 (1999).

[96] I. Berenstein, L. Yang, M. Dolnik, A. M. Zhabotinsky, and I. R. Epstein, Superlattice turing structures in a photosensitive reaction-diffusion system, Phys. Rev. Lett. 91, 058302 (2003).

[97] W. Pesch and G. Freund, Programm to check stability of forced rolls with wave vector $q_{f}$, unpublished work.

[98] G. Freund and W. Pesch, Convection with Modulation, unpublished work. 
[99] G. K. Auernhammer, D. Vollmer, and J. Vollmer, Oscillatory instabilities in phase separation of binary mictures: Fixing the thermodynamic driving, Journal of Chemical Physics 123, 134511 (2005).

[100] Y. Tasaka and Y. Takeda, Effects of heat source distribution on natural convection induced by internal heating, International Journal of Heat and Mass Transfer 48, 1164 (2005).

[101] G. Choblet and E. Parmentier, Thermal convection heated both volumetrically and from below: Implications for predictions of planetary evolution, Physics of the Earth and Planetary Interiors 173, 290 (2009), ISSN 00319201.

[102] W. Pesch, RBC convection with spatially modulated bulk heating, Technical Report (unpublished).

[103] G. G. Shaughnessy, Design of optical forcing experiments and construction of apparatus for Rayleigh-Bénard convection studies, Master's thesis, Cornell University (2004).

[104] M. Hirono, Band intensities of the 2.7 $\mu \mathrm{m}$ and $4.8 \mu \mathrm{m} \mathrm{CO}_{2}$ bands, Journal of the Physical Society of Japan 55, 3825 (1986).

[105] M. Hirono and T. Suda, Equivalent widths and band intensities of $\mathrm{CO}_{2}$, Applied Optics 29, 608 (1990).

[106] I. Opticts, International Scientific Product Catalog of optical Materials and Components (2008).

[107] M. Assenheimer and V. Steinberg, Rayleigh-Bénard convection near the gas-liquid critical point, Phys. Rev. Lett. 70, 3888 (1993).

[108] J. Oh and G. Ahlers, Thermal-noise effect on the transition to RayleighBénard convection, Phys. Rev. Lett. 91, 094501 (2003).

[109] B. P. Belousov, A periodic reaction and its mechanism, Sbornik Referatov po Radiatsionnoi Meditsine za 1958 god p. 145 (1958).

[110] V. Petrov, Q. Ouyang, and H. L. Swinney, Resonant pattern formation in a chemical system, Letters to Nature 388, 655 (1997).

[111] F. H. Fenton, S. Luther, E. M. Cherry, N. F. Otani, V. Krinsky, A. Pumir, E. Bodenschatz, and J. Gilmour, Robert F., Termination of Atrial Fibrillation Using Pulsed Low-Energy Far-Field Stimulation, Circulation 120, 467 (2009). 
[112] K. J. Åström and R. M. Murray, Feedback Systems (Princeton University Press, 2008).

[113] H. Haken, Brain dynamics: synchronization and activity patterns in pulsecoupled neutral nets with delays and noise (Springer, 2002).

[114] R. Manor, A. Hagberg, and E. Meron, Wave-number locking in spatially forced pattern-forming systems, Europhysics Letters 83, 10005 (2008). 



\section{Acknowledgments}

First, I want to thank my supervisor Prof. Bodenschatz for offering me this project and for supervising that work.

Special thanks goes to my friend and colleague Gabriel Seiden, who worked with me. The presented work here is a collaborative work which would not be what it is without him.

I also highly appreciate the support of Prof. Pesch from the University of Bayreuth. He developed large parts of the presented theory and wrote codes for simulations which were used to calculate several instability curves presented in this thesis. He always had an open ear for problems and has cross-read parts of the thesis.

Special thanks also to Mathieu Gibert, who gave me the feeling, that I can always knock at his door and ask questions about Fourier transform or the functioning of a Keithley multimeter. Especially during the writing process, discussions with him were very helpful to me.

I acknowledge Greg Bewley for cross-reading parts of the thesis and Annita for cross-reading and for pushing me, when I was lazy. Thanks goes to my brother Martin who also cross-read parts and who is always there for me.

It is also important to acknowledge Christian Westendorf with whom I met every Monday for the PIZZA-seminar at which we discussed recent developments in our work, in astrophysics and at the Georg-August University. This seminar was one of the highlight every week.

The three years in the Bodenschatz- group was a very nice time for me, which was especially due to all the new friends I gained and with whom I spend large parts of my leisure time. Thanks to the whole group!

And last but not least I thank my parents Elke and Berthold for a continuous support for more than 30 years. 